\title{
The Effect of Stratification on Wind-Driven, Cross-Shelf Circulation and Transport on the Inner Continental Shelf
}

\author{
by \\ Rachel Mandy Horwitz \\ B.A., Williams College, MA (2003) \\ Submitted in partial fulfillment of the \\ requirements for the degree of \\ Doctor of Philosophy in Physical Oceanography \\ at the \\ MASSACHUSETTS INSTITUTE OF TECHNOLOGY \\ and the \\ WOODS HOLE OCEANOGRAPHIC INSTITUTION \\ June 2012 \\ (c) Rachel Mandy Horwitz, 2012
}

The author hereby grants to MIT and to WHOI permission to reproduce and distribute publicly paper and electronic copies of this thesis document in whole or in part.

Author

Joint Program in Physical Oceanography Massachusetts Institute of Technology Woods Hole Oceanographic Institution

May 8, 2012

Certified by

Steven J. Lentz

Senior Scientist

Thesis Supervisor

Accepted by

Karl Helfrich

Chair, Joint Committee for Physical Oceanography 


\title{
The Effect of Stratification on Wind-Driven, Cross-Shelf Circulation and Transport on the Inner Continental Shelf
}

\author{
by \\ Rachel Mandy Horwitz \\ Submitted to the Joint Program in Physical Oceanography - Massachusetts \\ Institute of Technology / Woods Hole Oceanographic Institution \\ on May 8, 2012, in partial fulfillment of the \\ requirements for the degree of \\ Doctor of Philosophy in Physical Oceanography
}

\begin{abstract}
Observations from a three-year field program on the inner shelf south of Martha's Vineyard, MA and a numerical model are used to describe the effect of stratification on inner shelf circulation, transport, and sediment resuspension height. Thermal stratification above the bottom mixed layer is shown to cap the height to which sediment is resuspended. Stratification increases the transport driven by cross-shelf wind stresses, and this effect is larger in the response to offshore winds than onshore winds. However, a one-dimensional view of the dynamics is not sufficient to explain the relationship between circulation and stratification. An idealized, cross-shelf transect in a numerical model (ROMS) is used to isolate the effects of stratification, wind stress magnitude, surface heat flux, cross-shelf density gradient, and wind direction on the inner shelf response to the cross-shelf component of the wind stress. In well mixed and weakly stratified conditions, the cross-shelf density gradient can be used to predict the transport efficiency of the cross-shelf wind stress. In stratified conditions, the presence of an along-shelf wind stress component makes the inner shelf response to cross-shelf wind stress strongly asymmetric.
\end{abstract}

Thesis Supervisor: Steven J. Lentz

Title: Senior Scientist 


\section{Acknowledgments}

Thanks first to my advisor, Steve Lentz, who has been wonderful to work with for the past six years. He has been incredibly patient and completely available. He let me drag him into the world of sediment resuspension and transport, and while my thoughts tend to go many directions at once, Steve has guided me into a more focused and productive research path. Meeting with my committee has been an entirely positive experience. Rocky Geyer is so enthusiastic about all sorts of physics that every conversation with him has left me more excited about my own research. Peter Traykovski explained the sonar equation to me many times and has happily come along for this thesis which turned out not to have as much sediment transport in it as originally planned. Glenn Flierl has put up with some pretty terrible Skype and Internet connections and has always provided thoughtful, insightful feedback on any material I've sent to him.

Besides my committee, other members of the PO department and several scientists outside of WHOI have been a great help to me. Claudia Cenedese kindly agreed to chair my defense and even read my thesis. Her feedback will improve the clarity of my papers. Ken Brink has been my go-to for questions about ROMS and turbulence closure schemes, and provided MATLAB code for implementing Mellor-Yamada 2.0. Craig Marquette was incredibly helpful as I prepared instruments for deployment. He also found the largest paint stirrer I've ever seen for sediment calibrations in the lab. I ran my numerical model on two PO student computers that Julian Schanze keeps up and running. Thanks to him, my modeling project got off the ground remarkable quickly and smoothly. Chris Sherwood and Marinna Martini (USGS), and Gail Kineke (Boston College) loaned me optical backscatter sensors and let me keep them for a year and a half. Marinna also taught me how to use them. Larry Poppe (USGS) gave me workspace and instruction in his sediment lab. This thesis also relied heavily on data from the Martha's Vineyard Coastal Observatory.

In my first year as a grad student I noticed everyone thanked JP Headquarters 
at MIT and the WHOI Academic Programs Office and I thought it was some sort of formality. It isn't; I get it now. Thank you so, so much. Whenever I walk into either office with a problem, there's always someone to help me sort it out.

I've been in Woods Hole full or part time for the past eight and a half years so there are many people to thank who were less directly involved in me thesis. Britt Raubenheimer first brought me to Woods Hole as a Swashzone Student Fellow. She gave me a wetsuit and fins and sent me to work (play) in the crashing surf of San Diego, CA. She, Steve Elgar, and the whole NCEX crew made that fieldwork so much fun. Britt and Steve have continued to be great mentors throughout my time here in Woods Hole. Chris Sherwood kept me in Woods Hole when he hired me at USGS. During those two and half years, Woods Hole really started to feel like home.

I was lucky to have such great classmates enter the Joint Program with me. I never would have made it through the first two years of grad school without having Holly Dail, Cim Wortham, Rebecca Dell, and Pat Engel to work with. Nick Woods has been an awesome officemate and always waters the plants that I completely neglect. Elise Olson, Maya Yamato, Maya Bhatia, and all the WHOI-based JP students have made Woods Hole a great place to live and work.

My running buddies, Stace and Ann, were my first friends in Woods Hole. I have probably spent more cumulative hours with them than anyone else here. Leading by example, they've show me how to be a scientist, they wait for me when I am five minutes late for every single run, and they have become dear, dear friends. Chris Algar has made me smile though the most stressful parts of thesising. I am looking forward to whatever adventure we find next. Finally, my parents, Bruce and Lois Horwitz, and sister Suzanne Horwitz, have always been supportive. I am so glad they are my funny, nerdy, little family.

This work was supported through National Science Foundation grant no. OCE0548961, the WHOI Academic Programs Office, and the WHOI Coastal Ocean Institute. 


\section{Contents}

1 Introduction 13

2 The SWWIM experiment and the inner shelf south of Martha's $\begin{array}{ll}\text { Vineyard, Massachusetts } & 19\end{array}$

2.1 Field Program . . . . . . . . . . . . . . . . . . . . . . . . . . . . . 19

2.1.1 SWWIM hydrographic data . . . . . . . . . . . 20

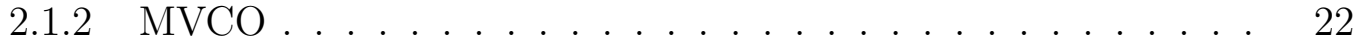

2.1 .3 SWWIM sediment data . . . . . . . . . . . . . . 22

2.1.4 Data from other sources . . . . . . . . . . . . . . 23

2.2 The Setting . . . . . . . . . . . . . . . . . . . . . 24

2.2.1 Bathymetry and bed composition . . . . . . . . . . . 24

2.2.2 Local Forcing: Wind, Waves, Heat Flux . . . . . . . . . 26

2.2 .3 Temperature and Salinity _ . . . . . . . . . . . . 27

2.2.4 Circulation, tides and bottom shear stress . . . . . . . . 34

3 The effect of stratification on sediment resuspension height $\quad 37$

3.1 Introduction . . . . . . . . . . . . . . . . . . 37

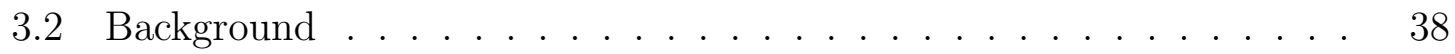

3.3 Field Program . . . . . . . . . . . . . . . . . . . . . . 40

3.4 Data Processing . . . . . . . . . . . . . . . . . . . . 41

3.4.1 Backscatter intensity from received ADCP echo intensity . . . 41 
3.4.2 Optical backscatter calibration to suspended sediment concentration $(\mathrm{SSC}) \ldots \ldots \ldots \ldots 4 \ldots \ldots \ldots$

3.4.3 Comparing Acoustic to Optical Backscatter . . . . . . . . . 46

3.5 Observations . . . . . . . . . . . . . . . . . 46

3.5.1 Example of two synoptic events . . . . . . . . . . . . . 47

3.5.2 Stratification reduces backscatter, independent of wave height 49

3.5.3 Height of resuspension follows mixed layer thickness . . . . . . 52

3.6 Theory and models for sediment suspension _. . . . . . . . . . . . 54

3.6.1 Bed shear velocity and particle size . . . . . . . . . . 54

3.6.2 Sediment concentration predictions from an eddy viscosity profile 57

3.6.3 Using Mellor-Yamada 2.0 . . . . . . . . . . . . . 58

3.6.4 Comparison to Mellor-Yamada 2.0 for unstratified conditions . 59

3.6.5 Comparison to M-Y 2.0 for stratified conditions . . . . . . . . 62

3.7 Implications for Transport . . . . . . . . . . . . . . . . . . . 63

3.7.1 Influence of changes in the sediment concentration profile . . . 63

3.7 .2 Influence of changes in the velocity profile . . . . . . . . 68

3.7.3 Mud transport near MVCO ............... 70

3.8 Summary . . . . . . . . . . . . . . . . . . . 71

3.A Appendix: Regressions between ADCP beams . . . . . . . . . 73

3.B Appendix: Optical and acoustic backscatter calibration . . . . . . . . 74

3.B.1 OBS in the field and lab . . . . . . . . . . . . . 74

3.B.2 Comparing ADCP to OBS . . . . . . . . . . 75

4 Observations of the effect of stratification on wind-driven cross-shelf $\begin{array}{ll}\text { circulation and transport on the inner shelf } & 77\end{array}$

4.1 Introduction . . . . . . . . . . . . . . . . . . . 77

4.1.1 Along-shelf wind stress background . . . . . . . . . . . 78

4.1.2 Cross-shelf wind stress background . . . . . . . . . . . . . 80

4.1 .3 This chapter . . . . . . . . . . . . . 81 
4.2 Data and Methods . . . . . . . . . . . . . . . . 82

$4.2 .1 \quad$ SWWIM . . . . . . . . . . . . . . . . 82

4.2 .2 Data processing . . . . . . . . . . . . . . . . . . 82

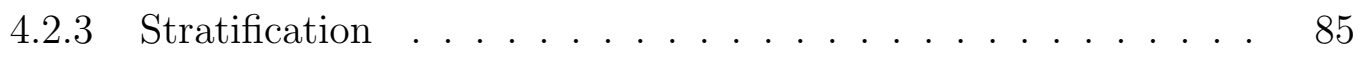

4.2 .4 Regressions with forcing terms . . . . . . . . . . . 90

4.2 .5 Quasi-1D Model . . . . . . . . . . . . . . . . . . . . 94

4.2 .6 Confidence Intervals _ . . . . . . . . . . . . . . . . 95

4.3 Along-shelf wind stress . . . . . . . . . . . . . . . 95

4.3 .1 Velocity regression profiles . . . . . . . . . . . . 995

4.3.2 Transport fraction . . . . . . . . . . . . . . . 98

4.3.3 Asymmetry between up- and downwelling wind stress . . . . 102

4.4 Cross-shelf wind stress _ . . . . . . . . . . . . . . . . 106

4.4 .1 Velocity regression profiles . . . . . . . . . . 106

4.4 .2 Transport fraction . . . . . . . . . . . . . . . 109

4.4 .3 Asymmetry . . . . . . . . . . . . . . . . . . . . 112

4.5 2D Dynamics . . . . . . . . . . . . . . . . . . . 117

4.5 .1 Mechanisms . . . . . . . . . . . . . . . . . 118

4.5.2 Comparing and combining forcing mechanisms . . . . . . . 123

$4.5 .32 \mathrm{D}$ analyses on a 3D inner shelf . . . . . . . . . . . . 129

4.6 Summary . . . . . . . . . . . . . . . . . . . . 133

4.A Appendix: Regressions with and without wave term . . . . . 135

5 Inner shelf response to cross-shelf wind stress in an idealized numer$\begin{array}{ll}\text { ical model } & 141\end{array}$

5.1 Introduction . . . . . . . . . . . . . . . . . . . 141

$5.2 \quad$ Numerical Model . . . . . . . . . . . . . . . . . . . . . . . . 142

5.2 .1 ROMS model . . . . . . . . . . . . . . . . . . . . . 143

$5.2 .2 \quad$ Base Case . . . . . . . . . . . . . . . . . . . . . 146

5.2 .3 Model runs . . . . . . . . . . . . . . . . . . . . . . . 147 
5.3 Scaling inner shelf circulation by basic forcing mechanisms: varying $N_{0}^{2}$ and $\tau^{x} \ldots \ldots \ldots \ldots \ldots \ldots \ldots$. . . . . . . . . . . . . . . 147

5.3 .1 Unstratified cases . . . . . . . . . . . . . . . . 151

5.3 .2 Initially stratified runs . . . . . . . . . . . . . . . . 154

5.3.3 Section summary ................... 162

5.4 The cross-shelf density gradient . . . . . . . . . . . . . . . . . 164

5.4.1 Predicting the cross-shelf density gradient . . . . . . . . . 165

5.4.2 The effect of a cross-shelf density gradient on transport . . . . 167

5.5 Surface Heat Flux . . . . . . . . . . . . . . . . . . . 170

5.5.1 Surface heat flux creates a cross-shelf density gradient . . . . . 172

5.5.2 Surface heat flux affects cross-shelf transport . . . . . . . . 175

5.5.3 Deep water boundary layer thickness . . . . . . . . . . . . . . 178

5.6 Combined cross- and along-shelf wind forcing . . . . . . . . . . 179

5.6.1 Asymmetric response to $\tau^{x}+\tau^{y}$ combined wind forcing . . . . 180

5.6.2 Example of equal parts $\tau^{x}$ and $\tau^{y}$ wind stress . . . . . . . . 184

5.6 .3 Defining the inner shelf . . . . . . . . . . . . 186

5.6.4 Stratification and transport for all wind directions and water

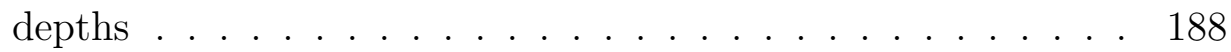

5.6.5 Growth of the inner shelf . . . . . . . . . . . . . . . . 190

5.7 Summary and Conclusion . . . . . . . . . . . . . . . . 191

5.A Appendix: Components of the combined cross- and along-shelf wind forcing . . . . . . . . . . . . . . . . . . . 195

6 Discussion and conclusions $\quad 197$

6.1 Discussion . . . . . . . . . . . . . . . . . . 197

6.1.1 Choosing an idealized numerical model . . . . . . . . . . . 197

6.1.2 Model results inform our interpretation of field observations . 199

6.2 Summary . . . . . . . . . . . . . . . . 203

6.2.1 Height of sediment resuspension . . . . . . . . . . . . . 204 
6.2.2 Observations of cross-shelf circulation . . . . . . . . . . . . . 204

6.2.3 A model of circulation . . . . . . . . . . . . . . . 205

6.3 In Conclusion . . . . . . . . . . . . . . . . . . 206 


\section{Chapter 1}

\section{Introduction}

Transport across continental shelves connects coastal ecosystems to the open ocean, and the inner shelf is a critical link in the cross-shelf transport pathway. Larvae hatched on rocky coastlines are carried offshore, and nutrients from deeper waters upwell near shore. Pollutants running off the land are transported away from the coast, and oil spilled at sea ends up as tar balls on beaches. All these processes depend on transport across the inner shelf, which is the region offshore of the surfzone where the surface and bottom boundary layers overlap. Wind stress is one of the main drivers of cross-shelf transport, and this thesis describes the influence of stratification on wind-driven transport across inner shelf.

Ekman (1905) described wind-driven circulation by allowing the Coriolis acceleration and a cross-shelf pressure gradient to balance both along- and cross-shelf wind stress components. His analytic solution for uniform density and eddy viscosity laid the groundwork to understand cross-shelf circulation driven by both along- and crossshelf wind stresses. A more detailed history of our understanding of transport driven by along-shelf (section 4.1.1) and cross-shelf (section 4.1.2) wind stresses is given in the introduction to Chapter 4. One key result from Ekman was that, in deep water, surface boundary layer transport is ninety degrees to the right of the wind stress. Accordingly, on mid and outer shelves, along-shelf winds typically drive cross-shelf transport and extensive observational and modeling work has focused on transport 
by upwelling and downwelling wind stresses. On the inner shelf, the along-shelf wind is less effective at driving cross-shelf transport in unstratified conditions because momentum mixes from the surface to the bottom before the Coriolis acceleration can turn it. The resulting divergence in the cross-shelf Ekman transport within the surface and bottom boundary layers leads to upwelling or downwelling on the inner shelf (left side of Figure 1-2).

Stratification extends the ability of along-shelf wind to drive cross-shelf circulation closer to shore (i.e., narrows the inner shelf), and increases the influence of the alongshelf wind stress on the inner shelf. On a homogeneous shelf, the response to upwelling and downwelling wind stress is symmetric, but on a stratified shelf, the response is asymmetric (Austin and Lentz, 2002). Downwelling circulation forces lighter under denser water, causing instability and mixing, which destratify the water column, while upwelling circulation brings up denser water below lighter water, increasing stratification and stability (Figure 1-1).

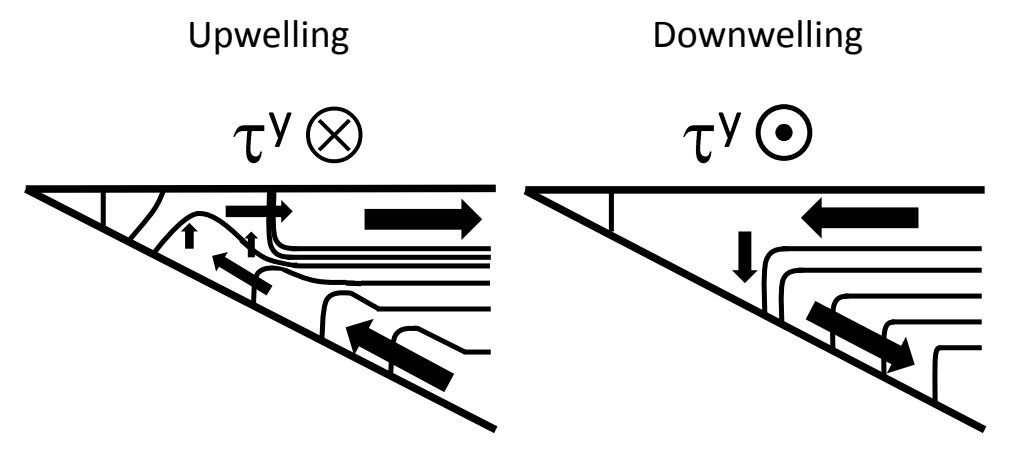

Figure 1-1: Cross-shelf circulation and ispoycnals resulting from upwelling (left) and downwelling (right) wind stresses over an initially stratified shelf.

For much of the intervening century since Ekman's seminal work, the influence of cross-shelf wind on shelf circulation has been largely ignored. Recent observational (Fewings et al., 2008) and modeling (Tilburg, 2003) studies have shown cross-shelf wind stress to be a significant mechanism for cross-shelf transport on the inner shelf. Over the inner shelf, a cross-shelf pressure gradient develops to oppose the crossshelf wind stress, and transport in the direction of the wind stress near the surface 
is balanced by a return flow in the lower portion of the water column (right side of Figure 1-2).

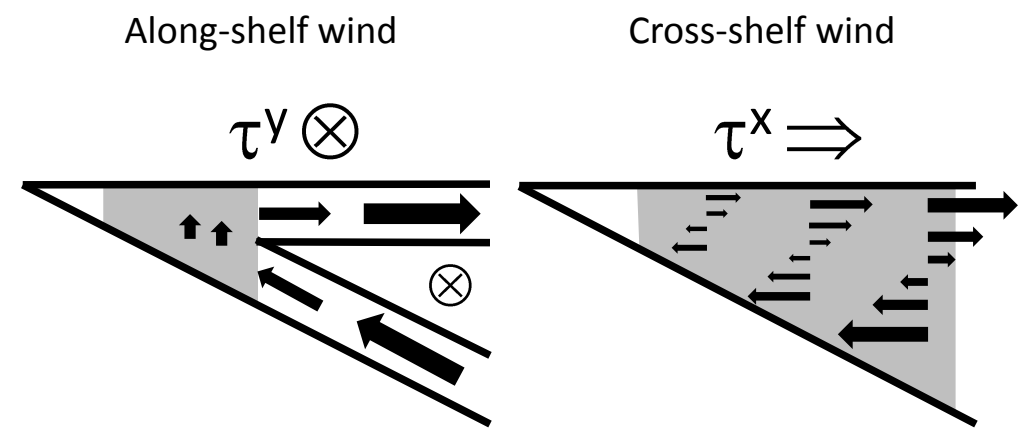

Figure 1-2: Cross-shelf circulation driven by along-shelf (left) and cross-shelf (right) wind stresses. Grey region indicates inner shelf, where surface and bottom boundary layers overlap.

Though continental shelves are typically stratified for much of the year, the inner shelf response to cross-shelf winds in stratified conditions is still unclear. South of Martha's Vineyard, MA, Fewings et al. (2008) noticed a more strongly sheared cross-shelf circulation during summer, when that field site is typically stratified, but Fewings et al. did not have density data to accompany the velocity measurements. Tilburg's (2003) model was initialized with stratification, but the wind stress mixed density and momentum to the same depth. All circulation was confined to the surface mixed layer so the water column was never stratified on the inner shelf (water depths shallower than the surface boundary layer thickness).

This thesis is motivated by the recent realization of the importance of cross-shelf winds at driving cross-shelf transport on the inner shelf combined with

1. the knowledge that this process occurs on stratified shelves (such as in summertime at Martha's Vineyard)

2. a near complete absence of observations of the phenomenon in stratified conditions, and

3. a lack of any model predicting how such an interaction might develop. 
This thesis investigates the effect of stratification on the inner shelf. Observations from the inner shelf south of Martha's Vineyard, MA and a numerical model are used to answer these questions:

1. How does stratification affect the vertical structure of the suspended sediment load?

2. How does stratification alter the inner shelf response to cross- and along-shelf wind stresses?

3. Which environmental factors determine the efficiency of cross-shelf wind at driving cross-shelf transport?

4. How does a stratified inner shelf develop?

The purpose of each chapter is outlined below.

\section{Chapter 2: The data set}

Chapter 2 describes the data sources for analyses in Chapters 3 and 4, and provides an overview of the measurements made on the inner shelf south of Martha's Vineyard, MA.

\section{Chapter 3: Height of sediment resuspension}

On the inner shelf, cross-shelf circulation often flows in one direction near the surface and the opposite direction near the bed so the height to which sediment is resuspended will affect how far and in what direction it is transported. While it is well established that stratification is a key factor in understanding sediment dynamics in estuaries and river plumes, far less attention had been paid to the effect of stratification on sediment resuspension height on the continental shelf. Using acoustic backscatter intensity from an ADCP as a proxy for sediment concentration, Chapter 3 describes the effect of stratification on the vertical structure of suspended sediment concentration on the inner shelf. 


\section{Chapter 4: Observations of circulation}

Prior to this investigation, Fewings et al. (2008) and Tilburg (2003) both demonstrated that cross-shelf winds generate stronger circulation than along-shelf winds on an unstratified inner shelf by driving surface transport in the direction of the wind stress, which is balanced by a pressure-gradient driven return flow lower in the water column. In addition, it has been established that stratification increases the efficiency of along-shelf wind at driving cross-shelf transport on the inner shelf. Chapter 4 uses field measurements of velocity, density, and wind stress from the inner shelf south of Martha's Vineyard, MA, to describe the effect of stratification on wind-driven, cross-shelf circulation and the variation of that effect with water depth, strength of stratification, and wind direction.

\section{Chapter 5: A model of circulation}

To resolve the discrepancy between our observations (Chapter 4) and previous modeling work (Tilburg, 2003), in Chapter 5, an idealized, 2D, cross-shelf transect in a numerical model (ROMS) is used to describe the effects of stratification, wind stress magnitude, surface heat flux, cross-shelf density gradient, and wind direction on the inner shelf response to the cross-shelf component of the wind stress. These factors are all present in the field but difficult to isolate in the observations.

\section{Chapter 6: Conclusion}

Chapter 6 discusses connections between the three scientific chapters and concludes with a summary of key results. 


\section{Chapter 2}

\section{The SWWIM experiment and the inner shelf south of Martha's Vineyard, Massachusetts}

Chapters 3 and 4 of this thesis are based on observations from the inner shelf off the southern coast of Martha's Vineyard, MA. This chapter describes the data sets used for these analyses and provides an overview of the hydrographic observations.

\section{$2.1 \quad$ Field Program}

The primary data set for this thesis is the group of six NSF funded SWWIM (Stratification, Wind, and Waves on the Inner shelf of Martha's Vineyard) study that included six deployments south of Martha's Vineyard, an island off the coast of Massachusetts (Figure 2-1(a)). Observations were made at four sites in 7, 12, 17.5, and 27.5 meters water depth, located $0.4,1.5,3.8$, and 11.1 kilometers from shore, respectively. Moorings measured temperature and salinity throughout the water column while collocated tripods held pressure sensors and upward-looking Acoustic Doppler Current Profilers (ADCP) that provided velocity and acoustic backscatter measurements. We also use the continuously available data from the Martha's Vineyard Coastal Observatory 
node for ADCP velocity, backscatter, and wave statistics and the Shore Mast and Air-Sea Interaction Tower (ASIT) for wind stress. Finally, three optical backscatter sensors provided data from one winter deployment at the 17-m site. Figure 2-1(a) shows the location of the field site on the US east coast and 2-1(b) shows the instrument location relative to Martha's Vineyard. In 2-1(b), color represents water depth and shows relatively uniform bathymetry in the along-shelf direction.

\subsubsection{SWWIM hydrographic data}

The six SWWIM deployments each span four to six months and are separated by one or two months. The first deployment, SWWIM2, started in October 2006 and the last deployment, SWWIM7, ended in February 2010. SWWIM1 was the pilot study and is not included here. The instrument turnaround times varied between sites, but the timing of the deployments roughly followed the schedule in Table 2.1.

Table 2.1: Schedule of SWWIM deployments

\begin{tabular}{lll}
\hline Deployment & Start Date & End Date \\
\hline \hline SWWIM2 & Oct. 06 & Apr. 07 \\
SWWIM3 & Apr. 07 & Oct. 07 \\
SWWIM4 & Oct. 07 & Apr. 08 \\
SWWIM5 & Apr. 08 & Nov. 08 \\
SWWIM6 & Nov. 08 & Jun. 09 \\
SWWIM7 & Jun. 09 & Feb. 10 \\
\hline
\end{tabular}

Each SWWIM deployment included tripods in 7, 17.5, and $27.5 \mathrm{~m}$ water depth and moorings at $7,12,17.5$, and $27.5 \mathrm{~m}$ depth along a cross shelf transect from two to twelve kilometers offshore (See Figure 2-1). The tripods each held an upward looking RDI WorkHorse Acoustic Doppler Current Profilers (ADCP) and a Seabird SEAGAUGE pressure sensor, and a Seacat CTD. The ADCP at $7 \mathrm{~m}$ had a frequency of $1200 \mathrm{kHz}$ and measured in $0.25 \mathrm{~m}$ bins starting at 1 meter above the bed (mab). The ADCPs at 17 and $27 \mathrm{~m}$ were $600 \mathrm{kHz}$ units and measured $0.5 \mathrm{~m}$ bins beginning at 2.25 mab, with reliable measurements ending at roughly $10 \%$ of the nominal water depth below the surface. Moorings near the tripods measured temperature roughly every $2.5 \mathrm{~m}$ throughout the water depth with alternating SeaBird MicroCats and 


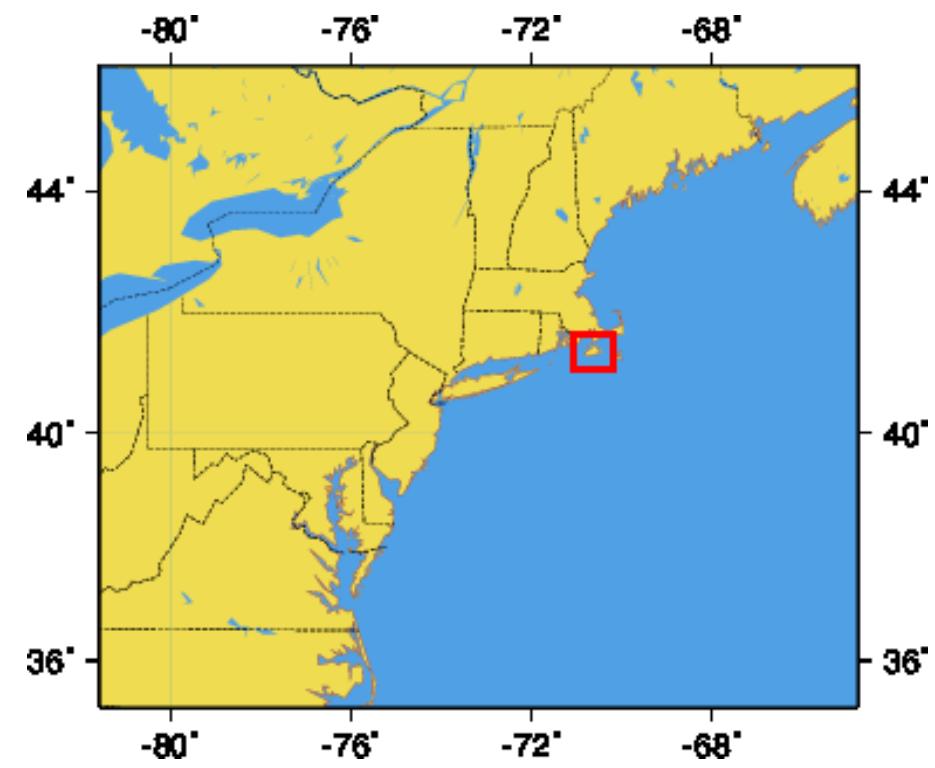

(a) US east coast with field site indicated in red, map made by USGS Map-It http://woodshole.er.usgs.gov/mapit

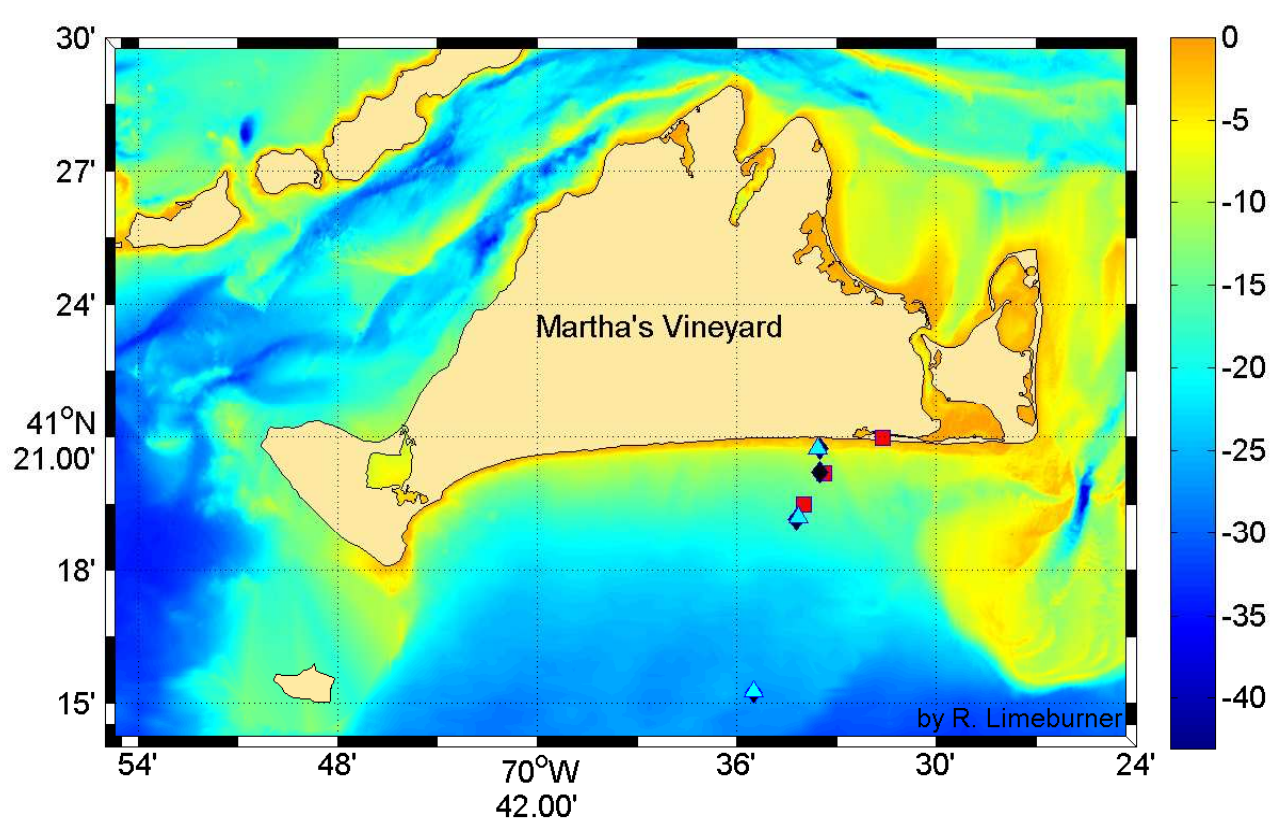

(b) Martha's Vineyard

Figure 2-1: Maps of field site and instrument locations. In (b), the colorbar indicates water depth; red squares locate the MVCO mast, node, and tower; Black diamonds represent TS moorings and blue triangles represent ADCP tripods. 
Onset TempPros. The MicroCats also measured conductivity at their $5 \mathrm{~m}$ spacing. The ADCPs also provided acoustic signal strength data for each bin with dependable results starting at $1.25 \mathrm{mab}$ at the $7-\mathrm{m}$ site and 2.75 mab at the deeper sites. The ADCPs collect data at $1 \mathrm{~Hz}$ for 5 minutes out of every twenty and record only the burst averages while calculating and recording wave spectrum data at $2 \mathrm{~Hz}$ for 10 minutes every four hours. The MicroCats sampled every 1.5 minutes and the TempPros sampled every 10 minutes. All temperature and conductivity data are interpolated onto the ADCPs twenty minute time base.

\subsubsection{MVCO}

The MCVO node continuously collects $2 \mathrm{~Hz}$ velocity and backscatter data from 12 $\mathrm{m}$ water depth in $0.5 \mathrm{~m}$ bins starting at 2.5 mab. There, the upward looking ADCP also estimates wave height, direction, and spectrum. The MVCO ASIT (Air-Sea Interaction Tower) provides temperature and conductivity at four depths in $15 \mathrm{~m}$ water as well as wind velocity measurements that we use to estimate wind stress from a bulk formula. Winds stress data also comes from the MVCO mast on South Beach on Martha's Vineyard, just onshore of the hydrographic array. These instruments are cabled to shore and collect data continuously. The MVCO data is provided as 20 minute averages. Data available online at www.whoi.edu/mvco/

\subsubsection{SWWIM sediment data}

Three Aquatec 200TY turbidity meters were deployed on the 17-m mooring on SWWIM6. They were located at 5, 7, and 13 meters above the bed and all sampled at $8 \mathrm{~Hz}$ for one second every 20 minutes, recording only the burst averages. On SWWIM5 and SWWIM6 cruises, bottom sediment samples were collected with a Van Veen grab sampler from the R/V Tioga. On September 11, 2008, three sam-

ples, roughly $100 \mathrm{~m}$ apart, were collected from each of the 7, 12, 27, 35, and 40-m isobaths. On November 12, 2008, three samples were collected at each of the 7 and 
17-m isobaths. On January 1, 2009, three samples were collected from each of the 12, 17 , and $27-\mathrm{m}$ isobaths.

Optical backscatter sensors were also deployed on the 17 and 27-m moorings and tripods during SWWIM5 and SWWIM6, but all except the three Aquatec OBS mentioned above failed to return useful data for reasons summarized below. During SWWIM5 and SWWIM6, a Seapoint turbidity meter was deployed on each of the 17 and $27-\mathrm{m}$ tripods at roughly $30 \mathrm{~cm}$ above the bed but the data quality was compromised by too high and too low fixed gain cables and by extensive fouling. During SWWIM5, three Aquatec 200TY Turbidity meters and one D\&A Instruments OBS$3 \mathrm{~A}$ were also deployed on the 17 and $27-\mathrm{m}$ moorings. The Aquatecs failed due to a bug in the AQUAtalk200TY v1.05 software that would not record data if bursts were programmed to last longer than one second. ${ }^{1}$ The alkaline batteries in the D\&A OBS-3A died before recovery and data was lost from the volatile memory. The D\&A OBS was destroyed in SWWIM6 when its lithium battery leaked.

\subsubsection{Data from other sources}

Several scientists involved with the nearby OASIS (Optics, Acoustics, and Stress In Situ) project have shared their data. OASIS took place at the MVCO Node in September or October of 2005, 2007, and 2009. Chris Sherwood provided bottom grab sample grain size distributions from approximately 50 sites in coarse and fine sand near the 12 and 16-m isobaths and Peter Traykovski provided a sonar survey showing bottom sediment type over most of this study area. In the 2007 OASIS experiment, Hill et al. (personal communication, 4 May 2010) used a LISST (Laser In Situ Scattering and Transmissometery), DFC (digital floc camera), and video settling column to measure particle size and fall velocity. During OASIS, an automated system also collected in situ water samples at $1.2 \mathrm{mab}$ in $12 \mathrm{~m}$ water depth. These data collectively inform our decisions on appropriate fall velocities and critical bed shear

\footnotetext{
${ }^{1}$ Aquatec reports that the problem does not exist in the new version of their turbidity meter, the AQUAlogger 210TY, and associated software.
} 
stresses for resuspension that are used in Chapter 3, section 3.7

\section{$2.2 \quad$ The Setting}

The inner shelf south of Martha's Vineyard experiences strong semidiurnal tides, synoptic scale wind and wave forcing and summertime stratification. The tides and wind-driven circulation have been described in detail by Fewings et al. (2008) and wave-driven flow is described by Lentz et al. (2008). We summarize those results here and elaborate on stratification, sediment and acoustic backscatter. For the purpose of describing seasonal variation, we define summer as April 1 to September 30 and winter as October 1 to March 31. These definitions are based on the observations of stratification described in section 2.2.2.

\subsubsection{Bathymetry and bed composition}

As shown in Figure 2-1, the bathymetry is relatively uniform in the alongshore direction for several kilometers around our transect. The cross-shelf bathymetry is shown in more detail in Figure 2-2, below. However, the large-scale bathymetry alone does not fully describe the shelf floor.

The bed of the inner shelf is characterized by rippled scour depressions of alternating fine and coarse sand. These bedform features are hundreds of meters wide and kilometers long and are mapped and described in detail by Goff et al. (2005) for a 5 $\mathrm{km}$ by $6.5 \mathrm{~km}$ area from the 8 to $18-\mathrm{m}$ isobaths that includes our 7,12 , and $17-\mathrm{m}$ sites. Bed samples from many locations around MVCO have been analyzed for grain size distributions. Mean and RMS values are available in Goff et al. (2005) and complete grain size distributions from Chris Sherwood (personal communication, 2 Sept 2008) provide the typical grain size distribution in fine patches of 98-99\% sand around 125 $\mu \mathrm{m}$ and 1-2\% mud by weight. On the coarse patches, the sediment is $>99 \%$ coarse sand $(125-1000 \mu \mathrm{m})$ with a small amount of mud. The 12-m mooring and 7-m mooring and tripod were on coarse sand. The 17-m sites and the MVCO node fall 


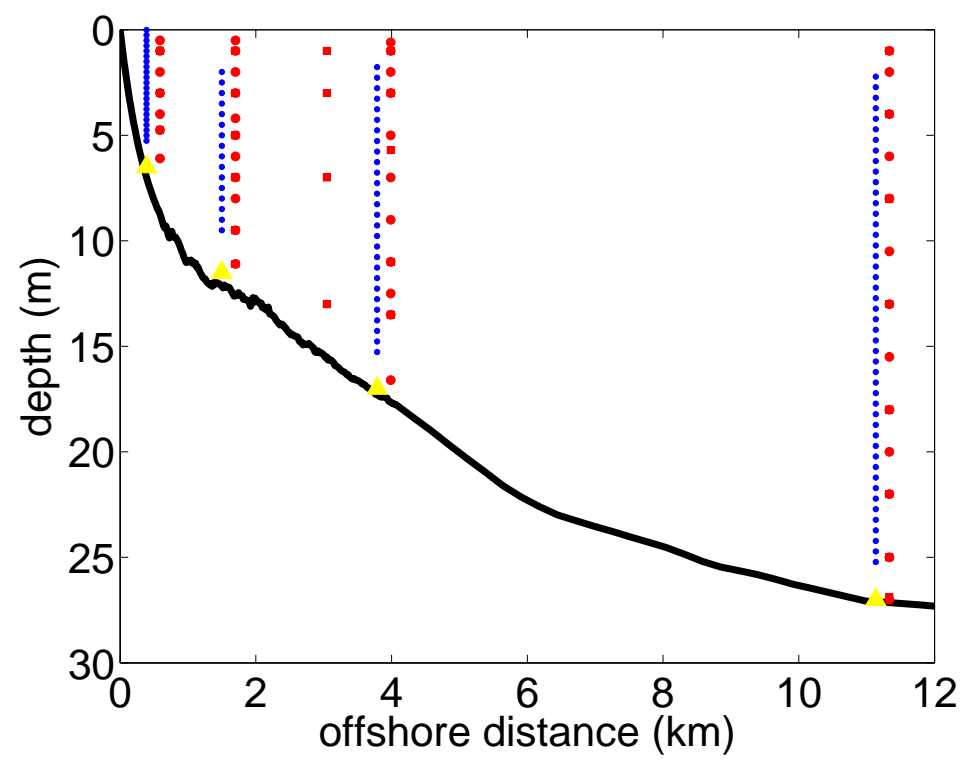

Figure 2-2: Cross-shelf bathymetry. Yellow triangles indicate locations of upward looking ADCPs, blue circles are location of ADCP velocity and backscatter measurements, red circles are locations of temperature measurements, and red squares indicate conductivity and temperature measurements.

in what Goff et al. mapped as a fine sand patch, however our tripods legs, mooring anchors, and bottom grab samples consistently indicated a significant mud fraction in the bed composition. The 27-m site is not on Goff et al.'s map but our bottom grab samples showed fine sand and mud.

Bottom grab samples collected on three deployment or recovery cruises provide a glimpse of the spatial and temporal variability of mud patches in areas of predominantly fine sand. Tripods sometimes sank into mud and had mud on them when recovered. Bottom grab samples from the $17-\mathrm{m}$ and $27-\mathrm{m}$ sites varied from all sand to mud overlaying sand to sand overlaying mud all within the top $15 \mathrm{~cm}$ of the bed. The order and thickness of the sand and mud layers varied between deployments months apart, but also over the $100 \mathrm{~m}$ between each of three grab samples collected along a single isobath at each mooring site. These sparse and anecdotal observations do not fully describe the evanescent mud patches, but do provide a hint that the mud patches vary over spatial scales of $100 \mathrm{~m}$ or less, and times scales of months or less. 


\subsubsection{Local Forcing: Wind, Waves, Heat Flux}

Wind and waves both vary on seasonal and synoptic scales, and both are larger in winter than summer. Surface heat flux varies most on the expected daily cycle that is superimposed on the same seasonal and storm specific variation as the wind and waves. Daily average values of significant wave height, wind stress, and surface heat flux spanning all SWWIM deplotments (October 2006 to February 2010) are shown in Figure 2-3.
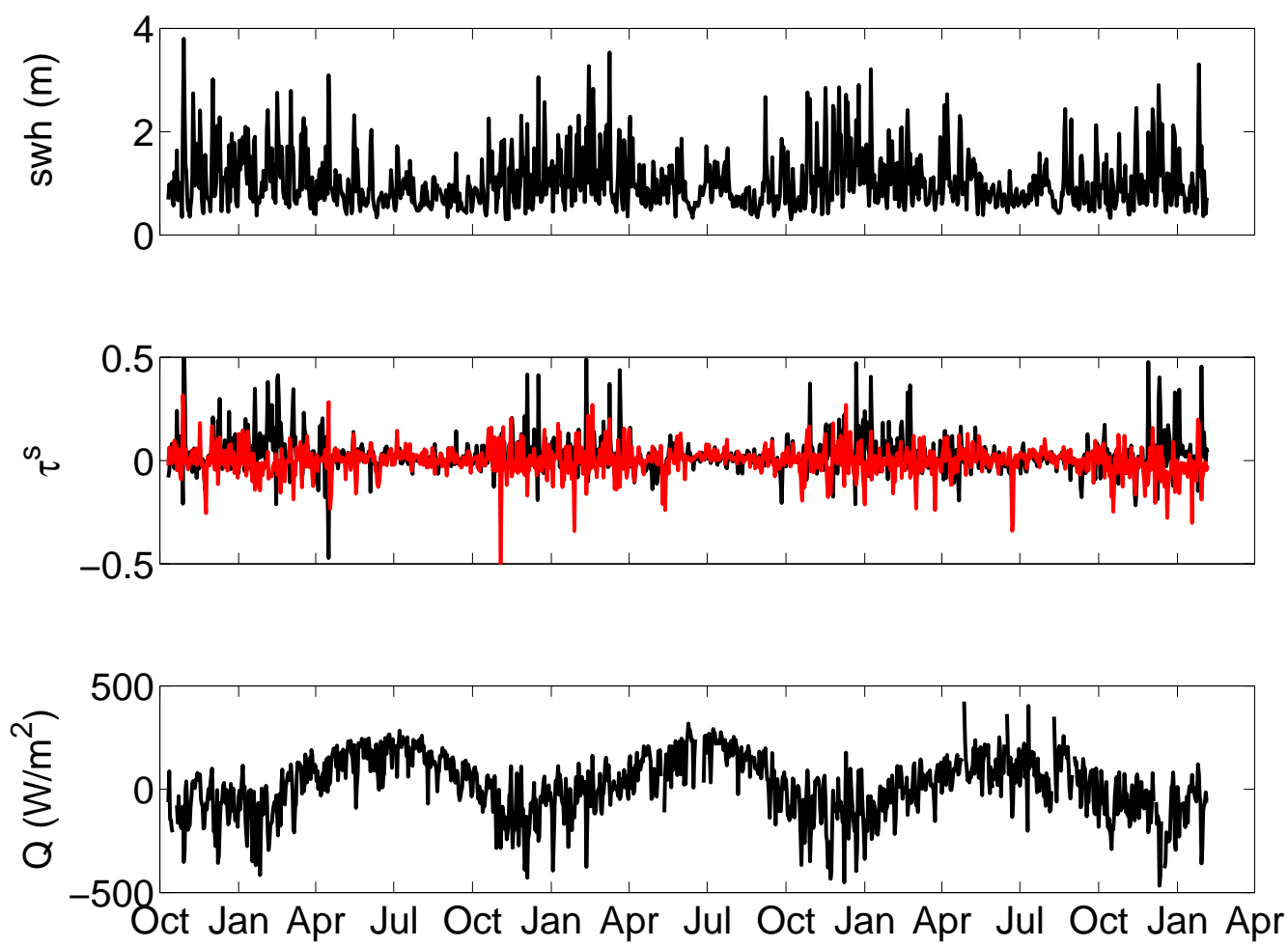

Figure 2-3: Top row: Daily average wave height. Middle row: wind stress (eastward, alongshore in black, northward and onshore in red). Bottom: surface heat flux, all for October 2006 through February 2010.

Summertime wind stress, $\tau^{s}$, averages $0.01 \mathrm{~Pa}$ towards the northeast $\left(\left|\tau^{s}\right|=0.06\right.$ $\mathrm{Pa}$ with $0.08 \mathrm{~Pa}$ standard deviation), while average winter wind stress is $0.05 \mathrm{~Pa}$ towards the east-southeast $\left(\left|\tau^{s}\right|=0.10 \mathrm{~Pa}\right.$ with $0.13 \mathrm{~Pa}$ std). Histograms of winter (left) and summer (right) wind stress magnitude and direction are shown in the top row of Figure 2-4 and wind stress vectors averages over each month of the year are 
shown in the bottom row.

Wind stress magnitude is correlated with significant wave height $(|R|=0.54,>$

$99 \%$ confidence) with the highest correlation for northward (onshore) wind stress $(|R|$ $=0.72,>99 \%$ conf. for wind $\pm 30^{\circ}$ of North) and lowest correlation for southward (offshore) stress $\left(|\mathrm{R}|=0.20,98 \%\right.$ conf. for wind $\pm 30^{\circ}$ of South) due to the short fetch from the south side of Martha's Vineyard to the node. Driven by larger wind stresses, the waves are also typically larger and more variable in the winter with $1.1 \mathrm{~m}$ mean, $0.65 \mathrm{~m}$ std for significant wave height in the winter and $0.89 \mathrm{~m}$ mean and $0.42 \mathrm{~m}$ std in the summer. The average wave period is 5.5 seconds in summer and $5.6 \mathrm{~s}$ in winter. Winter and summer significant wave height and direction are shown in the second row of Figure 2-4.

Surface heat flux is usually positive during the day and negative at night all year, with daily averages of $-64 \mathrm{~W} / \mathrm{m}^{2}$ over the winter (Oct. - Mar.) and $121 \mathrm{~W} / \mathrm{m}^{2}$ in summer (Apr. - Sept.) The annual average is $40 \mathrm{~W} / \mathrm{m}^{2}$. Figure $2-5$ shows the average daily cycle of surface heat flux for winter and summer as well as yearday averages over our three-year data set.

\subsubsection{Temperature and Salinity}

With no major freshwater sources from Martha's Vineyard, temperature is the main control of stratification, especially in the summer. Salinity ranges from 31 in the summer to 33 in the winter and spring, with up to a few tenths PSU variation in the vertical. Temperature varies from near $0{ }^{\circ} \mathrm{C}$ in the winter to over $20{ }^{\circ} \mathrm{C}$ in the summer with several degrees variation in the vertical typical in summertime (Figure 2-6). At the 7-m site, one or two degrees difference is common and at the 27-m site the difference stays over 2 degrees nearly all summer peaking near 10 degrees (Figure 2-7). This thermal stratification varies over tidal, daily, and synoptic scales. Fewings and Lentz (2011) describes the heat budget at MVCO and how, on seasonal time scales, advection due to upwelling balances the summertime surface heat flux. In the summer, at the 7 and 12-m sites, mixed and stratified water pass by every 

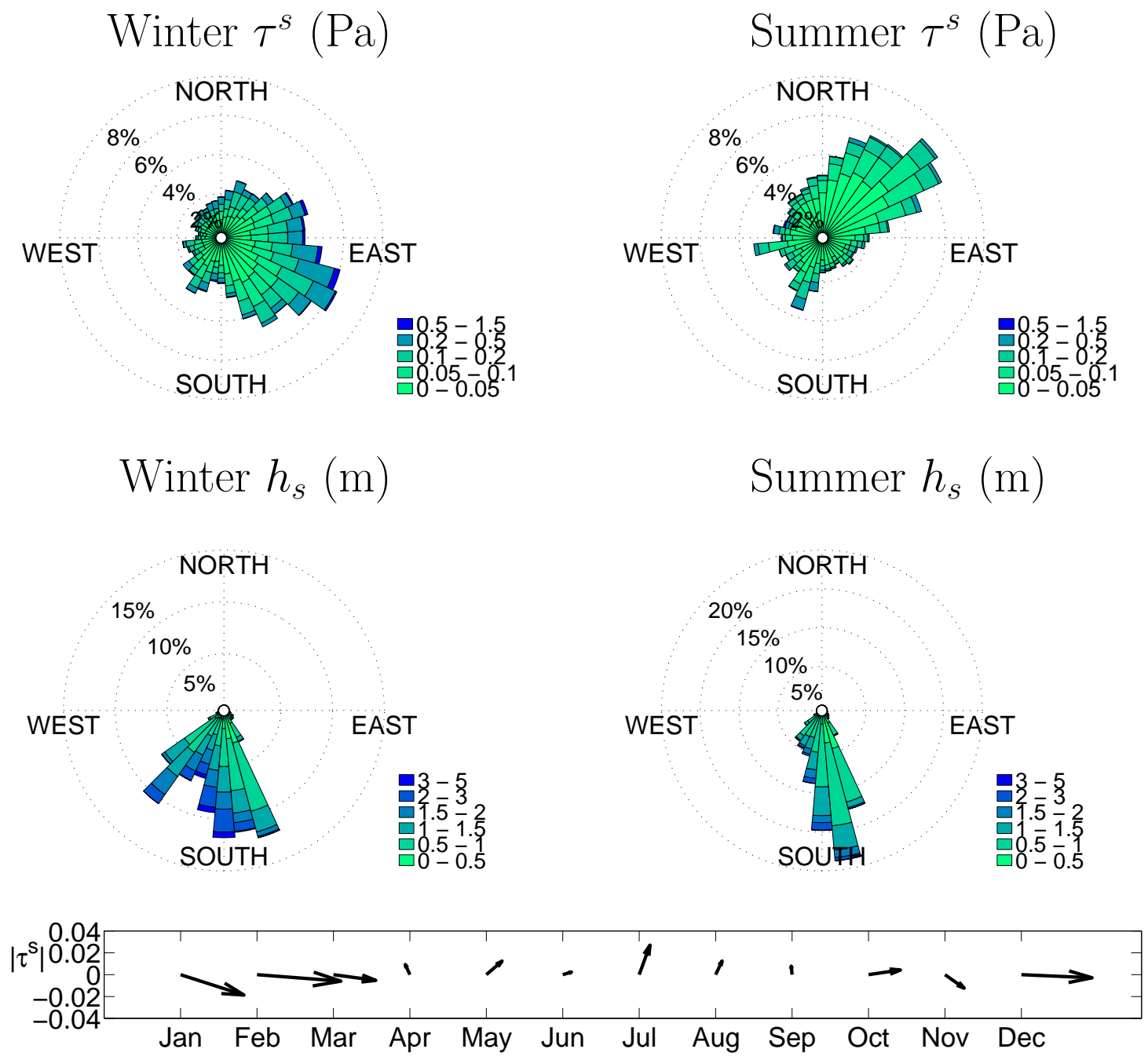

Figure 2-4: Histograms of direction for wind stress (top row) and dominant wave direction (second row) with color indicating distribution of wind stress magnitude $(\mathrm{Pa})$ and significant wave height $(\mathrm{m})$ for winter (left) and summer (right) conditions. Bottom row shows vectors of monthly average wind stress $(\mathrm{Pa})$ at the ASIT.

tidal cycle while the 17 and $27-\mathrm{m}$ sites remain stratified over the entire tidal cycle. Fall storms mix the entire water column at all four sites and wintertime storms and surface cooling keep the temperature vertically uniform until spring. Figure 2-6 shows typical winter (left) and summer (right) temperatures at 7, 12, 17, and $27 \mathrm{~m}$ water depth for one week each in February and July 2008. Note the different winter and summer temperature scales.

In the summer, there is a large cross-shelf variation in the structure of the stratifi- 


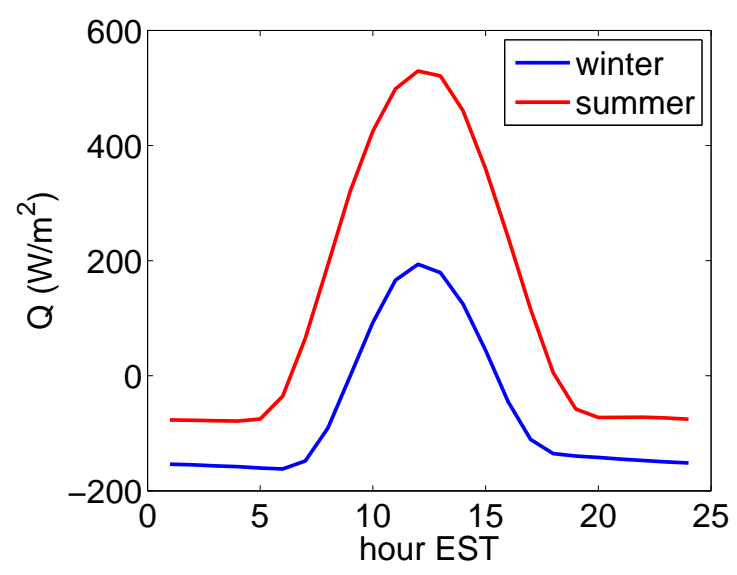

(a) daily cycle

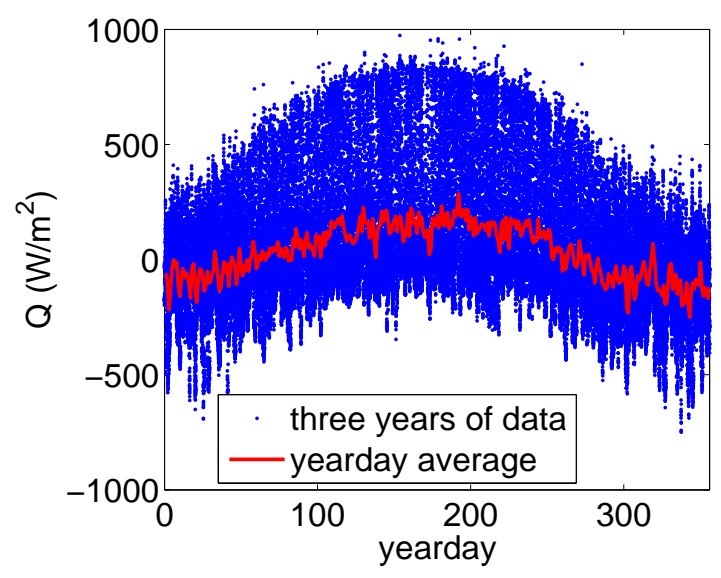

(b) annual cycle

Figure 2-5: Average daily cycle of surface heat flux for winter (blue) and summer (red) in on the left. Yearday averages (red) overlaying three years of surface heat flux data (blue) on the right.

cation, which can be seen in the temperature plots in the right column of Figure 2-6. At the $7-\mathrm{m}$ site, the temperature throughout the water column varies with the tide, and the stratification appears and disappears each cycle. The strong tidal currents mix much of the water column at this shallow depth. The 12 and 17-m sites show increasing stratification that is still occasionally mixed away. At the $27-\mathrm{m}$ site, there are tidal fluctuations in the height of particular isotherms, but the stratification persists. Figure 2-7 shows the full time series of top-to-bottom temperature differences. All four sites show near-zero vertical variation in temperature in the winter and increasing stratification throughout the summer. The maximum (summertime) temperature difference is smallest at the $7-\mathrm{m}$ site and largest at the $27-\mathrm{m}$ site. The $27-\mathrm{m}$ site and to a lesser extent, the 17 -m site, does not fully mix for several consecutive months in the summer.

Several methods of defining the summer season and stratified conditions yield similar results. Figure 2-8 shows a three year time-series of the top-to-bottom temperature difference at the $17-\mathrm{m}$ mooring. Vertical black lines indicate April $1^{\text {st }}$ and October $1^{\text {st }}$ of each year, which we've used to divide summer and winter seasons. Red dots indicate times when the water column is stratified $\left(\Delta \mathrm{T}>0.1^{\circ} \mathrm{C} / 0.5 \mathrm{~m}\right.$ somewhere 

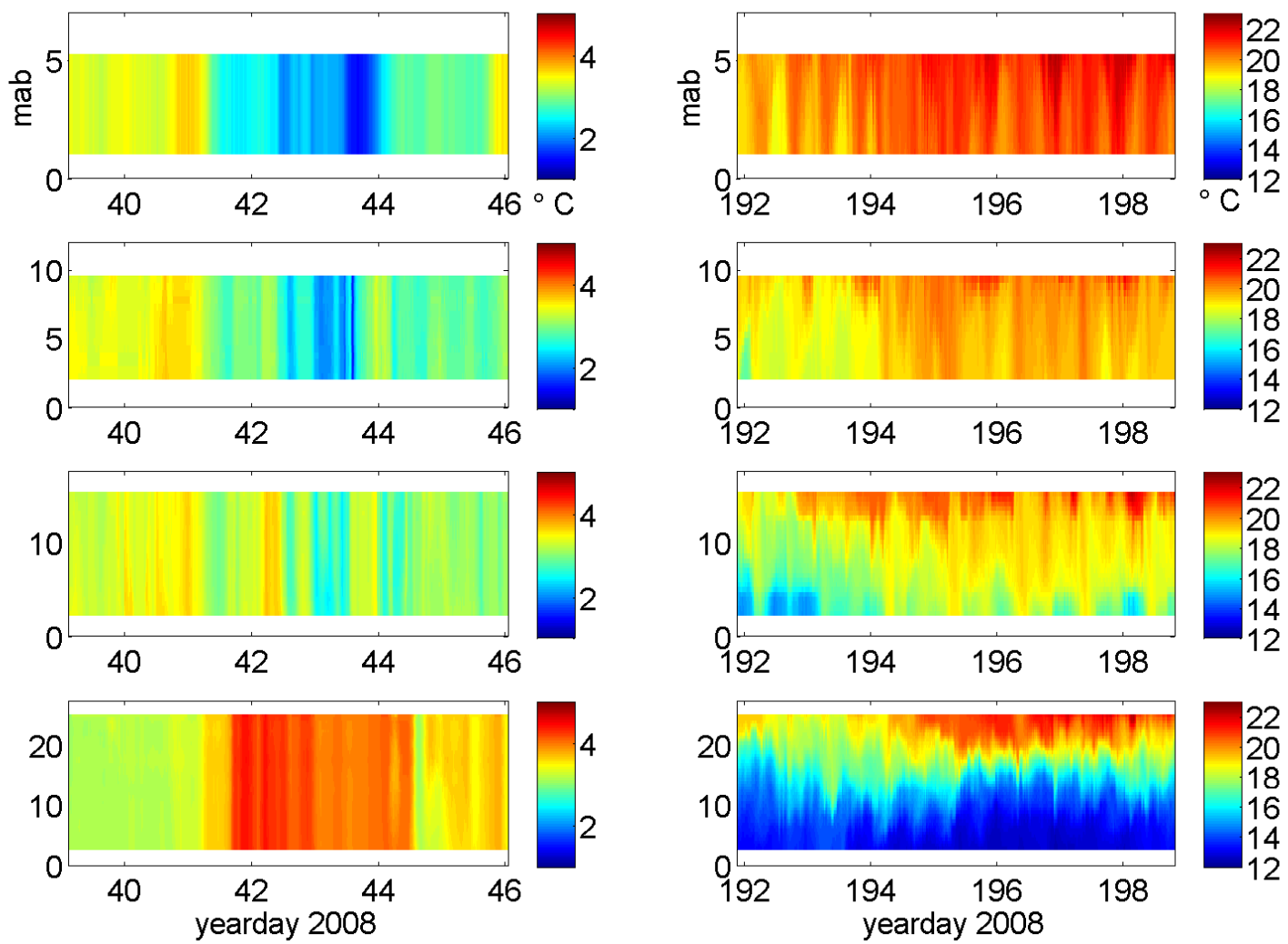

(a) winter

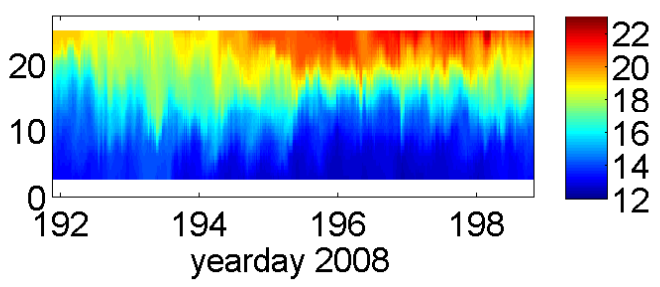

(b) summer

Figure 2-6: Temperature from all four mooring sites for a typical week in winter and summer.

in profile, as defined in Chapter 3, section 3.5). Time series from other mooring sites (not shown) have similar seasonal transitions.

Differences in temperature and salinity between the highest and lowest CTDs were compared to the resulting density difference. Multiple regression analyses show that variances in stratification predicted from vertical temperature differences are two to twenty times larger than the variances in stratification predicted from the regressions of vertical salinity differences. Table 2.2 shows the variance of stratification and of the regression predictions for stratification by temperature and salinity differences at each of the 7, 12,17, and 27-m sites. Regression slopes for temperature, $\mathrm{T}$, and salinity, S, are denoted $b_{t}$ and $b_{s}$.

In winter, surface cooling and offshore advection of colder, shallower water over 

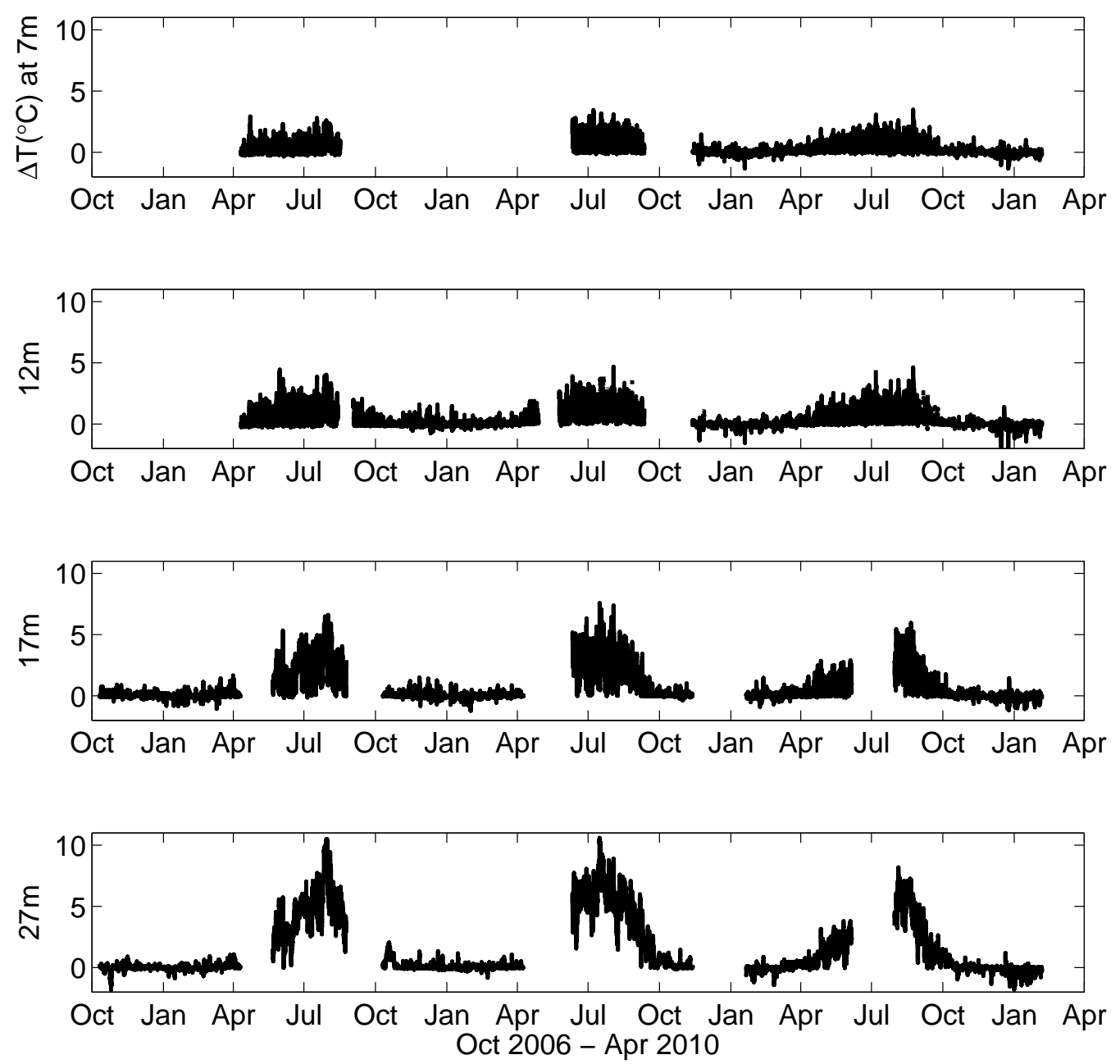

Figure 2-7: Time series of top to bottom temperature difference at 7, 12, 17, and 27-m sites.

Table 2.2: Variance of density difference and of regression predictions for density difference by temperature and salinity difference

\begin{tabular}{llll}
\hline Site & $\operatorname{var}\left(\Delta \sigma_{\theta}\right)$ & $\operatorname{var}\left(b_{t} * \Delta \mathrm{T}\right)$ & $\operatorname{var}\left(b_{s} * \Delta \mathrm{S}\right)$ \\
\hline \hline $7-\mathrm{m}$ & 0.0177 & 0.0104 & 0.0052 \\
$12-\mathrm{m}$ & 0.0303 & 0.0221 & 0.0048 \\
$17-\mathrm{m}$ & 0.1126 & 0.0906 & 0.0050 \\
$27-\mathrm{m}$ & 0.3790 & 0.2343 & 0.0272 \\
\hline
\end{tabular}

deeper, warmer water leads to temperature inversions. These are either unstable and soon mix away or can be maintained by stable salinity structure. However, the 


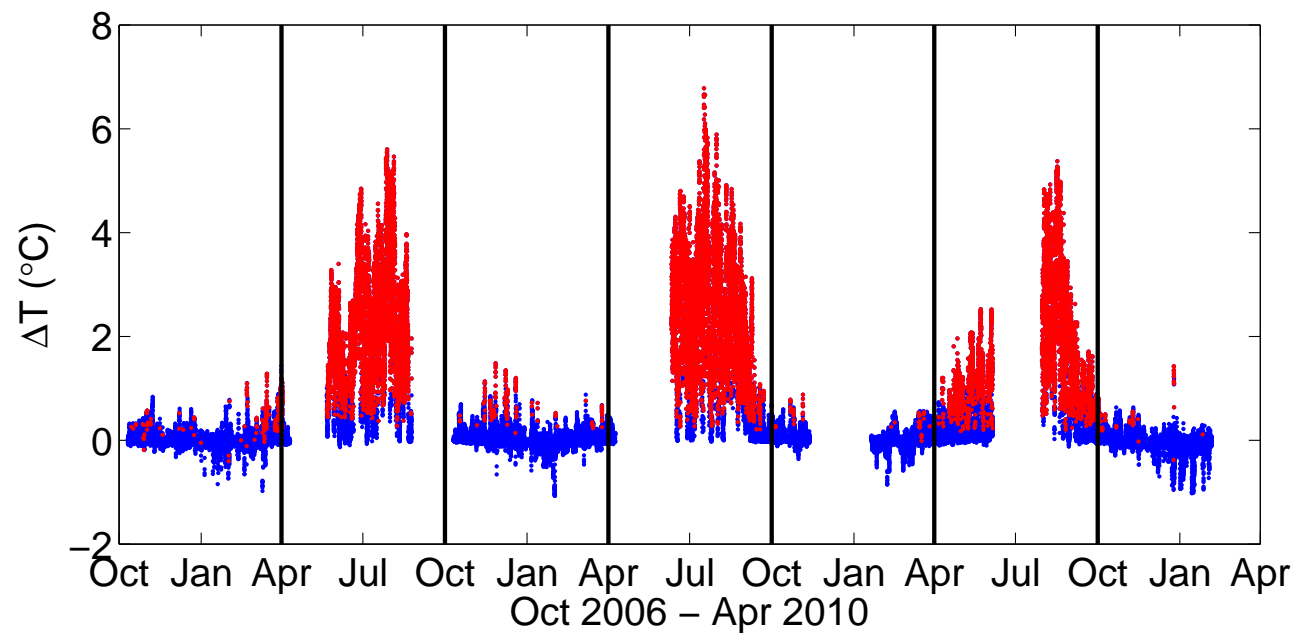

Figure 2-8: Time series of top to bottom temperature difference at 17-m site. Red dots indicate times defined as stratified for analysis in Ch. 3, sections 3.5 and 3.6. Vertical black lines mark April $1^{\text {st }}$ and October $1^{\text {st }}$ of each year, dividing summer and winter seasons

stratification in these cases is still an order of magnitude smaller than summertime thermal stratification with up to $0.05-0.1 \mathrm{~kg} / \mathrm{m}^{3}$ top to bottom density difference $\left(\mathrm{N}^{2} \approx 10^{-5}\right.$ to $\left.10^{-4} \mathrm{~s}^{-2}\right)$ due to salinity compared to $1-2.5 \mathrm{~kg} / \mathrm{m}^{3}\left(\mathrm{~N}^{2} \approx 10^{-3}\right.$ $\mathrm{s}^{-2}$ ) from temperature at the 7 to $27-\mathrm{m}$ sites. Our temperature data coverage is higher resolution and more complete than for salinity so we use temperature in lieu of density for analysis in Chapter 3 where we focus on times that are highly stratified. In Chapter 4 we return to using the full density field, which allows us to estimate the thermal wind shear from the cross shelf density gradient in the winter, when cross shelf temperature gradient would predict the opposite direction shear from the salinity gradient. Time series of stratification at the four mooring sites are shown in Figure 2-9. Stratification is calculated from the slope of a linear fit to the density measurements for times when two or more measurements are available at a given mooring.

Cross-shelf gradients of surface density, $\Delta \sigma_{\theta} / \Delta x\left(\mathrm{~kg} / \mathrm{m}^{4}\right)$, between three pairs of moorings are shown in Figure 2-10. Note vertical axis limits are different for the three time series. These density gradients are calculated using the topmost density measurement at each mooring. Because the density data have gaps at different times 
at different moorings, $\Delta \sigma_{\theta} / \Delta x$ values are sparser than the density measurements at any one mooring alone. In Figure 2-10, the data are plotted as a function of yearday, combining the full SWWIM time series to show a single annual cycle. The cross shelf density gradient is generally positive in all seasons, but larger and more variable in summertime. The largest gradients are found between the 7 and $12 \mathrm{~m}$ sites.
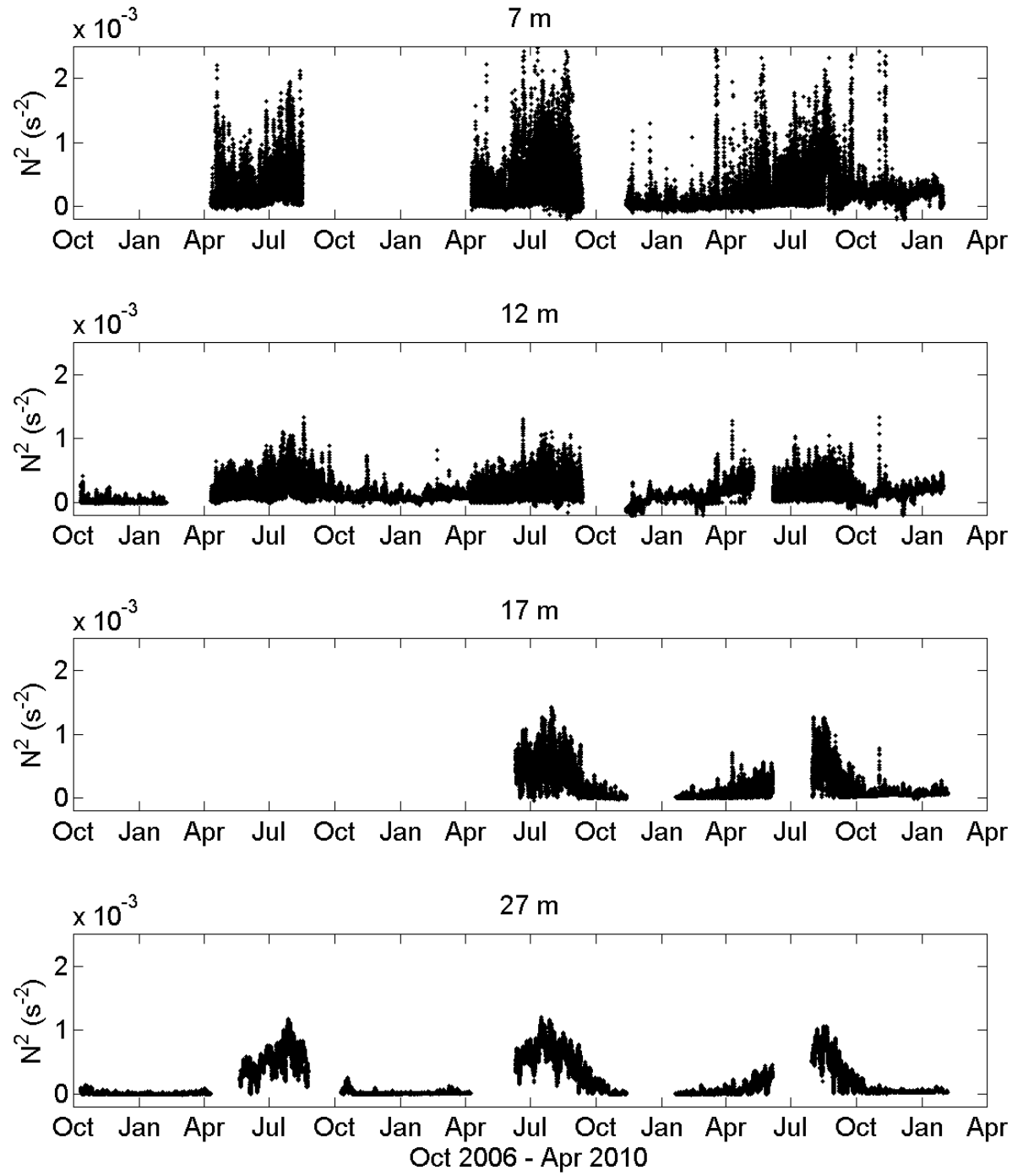

Figure 2-9: Time series of stratification at 7, 12, 17, and 27-m sites. 

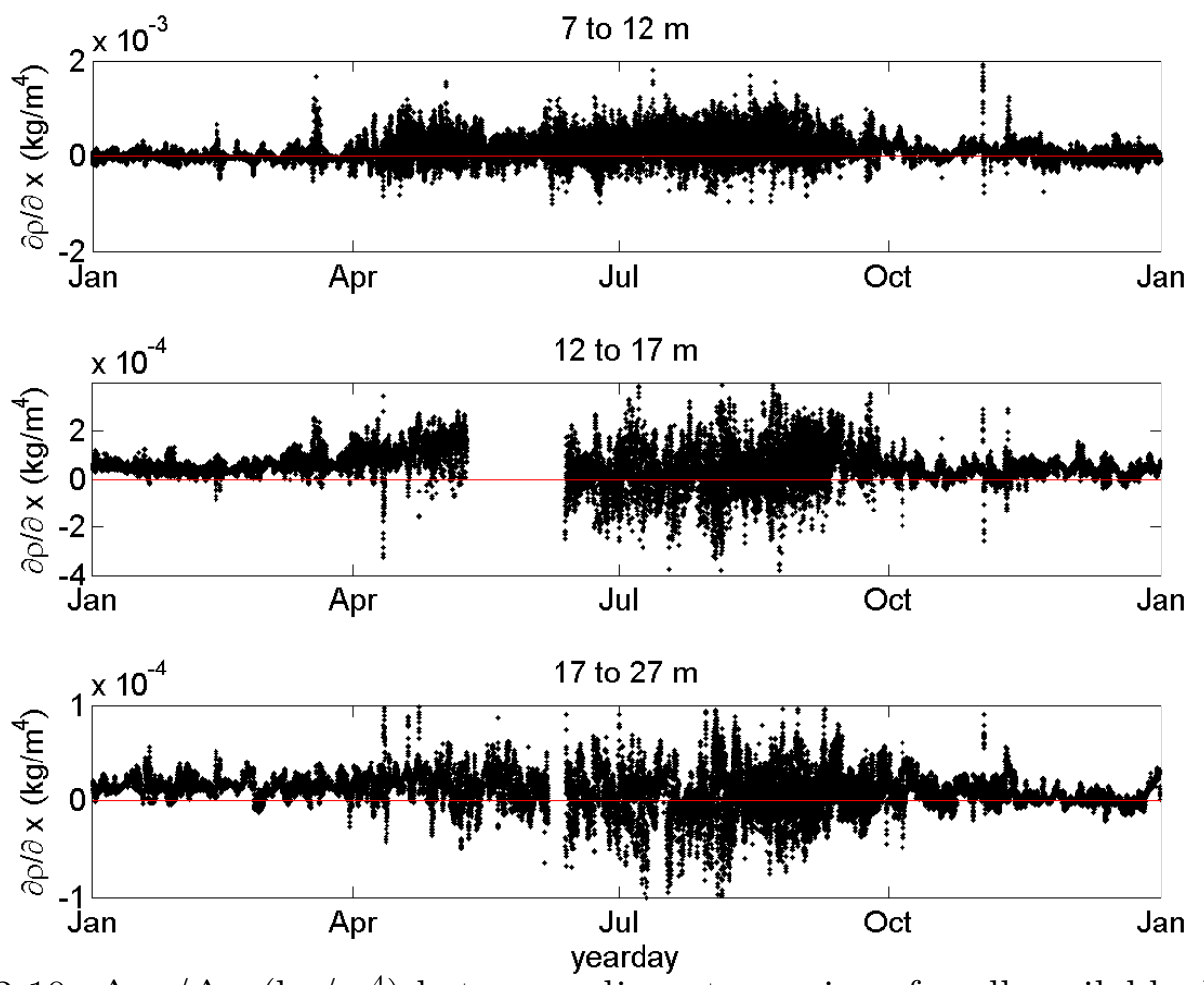

Figure 2-10: $\Delta \sigma_{\theta} / \Delta x\left(\mathrm{~kg} / \mathrm{m}^{4}\right)$ between adjacent moorings for all available data Oct 2006 - Apr 2010, as a function of yearday.

Table 2.3: Seasonal averages of cross-shelf gradients of surface density, $\Delta \sigma_{\theta} / \Delta x$, between moorings

\begin{tabular}{llll}
\hline Moorings: & $\begin{array}{l}7 \text { to } 12-\mathrm{m} \\
\Delta \mathrm{x}:\end{array}$ & $\begin{array}{l}12 \text { to } 17-\mathrm{m} \\
2200 \mathrm{~m}\end{array}$ & $\begin{array}{l}17 \text { to } 27-\mathrm{m} \\
7500 \mathrm{~m}\end{array}$ \\
\hline \hline $\begin{array}{l}\text { Summer } \\
\text { (Apr. - Sept.) }\end{array}$ & $2.4 \times 10^{-4} \pm 1.4 \times 10^{-6}$ & $4.1 \times 10^{-5} \pm 7.3 \times 10^{-7}$ & $1.1 \times 10^{-5} \pm 1.8 \times 10^{-7}$ \\
$\begin{array}{l}\text { Winter } \\
\text { (Oct. - Mar.) }\end{array}$ & $1.7 \times 10^{-5} \pm 8.4 \times 10^{-7}$ & $4.5 \times 10^{-5} \pm 2.5 \times 10^{-7}$ & $1.1 \times 10^{-5} \pm 7.6 \times 10^{-8}$ \\
\hline
\end{tabular}

All values are $\mathrm{kg} / \mathrm{m}^{4}$

\subsubsection{Circulation, tides and bottom shear stress}

Tides on the inner shelf south of Martha's Vineyard are dominated by the M2 constituent. The major axis of the depth-averaged M2 tidal ellipses are roughly alongisobath and have magnitudes of $18,23,20$, and $16 \mathrm{~cm} / \mathrm{s}$ at the $7,12,17$, and $27-\mathrm{m}$ sites respectively. The minor (onshore) components have magnitudes of $1,3,4$, and 8 $\mathrm{cm} / \mathrm{s}$. The full tidal circulation is slightly larger, reaching maximum speeds of 28,35 , 
33 , and $31 \mathrm{~cm} / \mathrm{s}$ at the $7,12,17$, and $27-\mathrm{m}$ sites. Tidal constituents were calculated using T_TIDE in MATLAB (Pawlowicz et al., 2002). A more detailed analysis of tidal variability near MVCO is given by Shearman and Lentz (2004).

The depth averaged mean flow is thought to be at least partially due to tidally rectified flow (Ganju et al., 2011). The annual mean is $2-3 \mathrm{~cm} / \mathrm{s}$ westward at the 12,17 , and $27-\mathrm{m}$ sites and slightly $(0.7 \mathrm{~cm} / \mathrm{s})$ eastward at the $7-\mathrm{m}$ sites. At all four sites, the flow is more strongly westward in the summer than the winter, while the standard deviations in the flow are larger in the winter (Table 2.4).

Table 2.4: Means and standard deviations of the eastward (roughly along-shelf), depth-averaged, subtidal flow

\begin{tabular}{lllll}
\hline Site & Winter mean & Winter STD & Summer mean & Summer STD \\
\hline \hline $7-\mathrm{m}$ & 0.031 & 0.086 & -0.026 & 0.059 \\
$12-\mathrm{m}$ & -0.017 & 0.078 & -0.061 & 0.046 \\
$17-\mathrm{m}$ & -0.011 & 0.076 & -0.047 & 0.047 \\
$27-\mathrm{m}$ & -0.0015 & 0.061 & -0.079 & 0.056 \\
\hline All values are $\mathrm{m} / \mathrm{s}$ & & &
\end{tabular}

Over synoptic time scales, cross and along-shelf winds and waves drive circulation of several centimeters per second. The standard deviations (see Table 2.4) of the along-shelf subtidal flow are smaller than the standard deviation tidal velocities, but the maximum values seen during storms are larger than the maximum tidal currents. Maximum depth average velocities peaked at $94,51,50$, and $44 \mathrm{~cm} / \mathrm{s}$ at the 7 to $27-\mathrm{m}$ sites in storms during SWWIM.

The relative strengths and vertical structures of wind and wave driven circulation during unstratified conditions are described in detail by Lentz et al. (2008) and Fewings et al. (2008), respectively, and the effect of stratification on these types of circulation is explored in Chapter 4 of this thesis. The summertime stratification described in section 2.2.3 also allows internal tides to develop, adding to the vertical structure of summertime wind and wave driven circulation.

For the sediment suspension discussed in Chapter 3, the most important feature of the velocity is the bottom shear stress it generates. Both mean currents and wave orbital velocities contribute to the bottom shear velocity, but their relative 
importance varies with water depth. Wave-, current- and wave-current-shear stresses were estimated following Madsen's (1994) iterative method. At $7 \mathrm{~m}$ depth, the wave contribution is always larger. At $12 \mathrm{~m}$, the current shear velocity is larger than the wave shear velocity $5 \%$ of the time. At $17 \mathrm{~m}$, the current shear velocity is larger than the wave shear velocity $19 \%$ of the time. Finally, at $27 \mathrm{~m}$, the current shear velocity is larger a majority, $57 \%$, of the time. 


\section{Chapter 3}

\section{The effect of stratification on sediment resuspension height}

\subsection{Introduction}

The inner shelf is the region outside the surfzone where the upper and lower boundary layers overlap. As the link between the surfzone and midshelf, the inner shelf plays an important role in determining the fate of the sand and mud that pass through it. Stratification is a common feature of many continental shelves and often extends into the inner shelf, but observational studies have rarely captured the interaction between sediment concentration and the thermocline on the shelf (Glenn et al., 2008 is one exception).

Stratification increases the shear in the cross-shelf circulation's response to crossshelf winds (Fewings et al., 2008), along-shelf winds (Lentz, 2001), and waves (Lentz et al., 2008). At the same time, stratification inhibits the vertical mixing that would otherwise move momentum and particulate matter throughout the water column. The combination of these two features makes it possible for stratification to affect the net sediment transport by preventing suspended sediment from reaching the upper part of the water column where the cross-shelf flow may be in the opposite direction from the lower part of the water column (Fewings et al., 2008). 
This chapter takes aim at one part of the larger problem of understanding sediment transport on the inner shelf. We focus on the effect of stratification on the vertical structure of suspended sediment. This observational study is based on a cross-shelf transect from the inner shelf south of Martha's Vineyard, MA, near the Martha's Vineyard Coastal Observatory (MVCO). Three years of moored current, temperature,

conductivity, acoustic and optical backscatter, wave and wind measurements spanning a wide range of weather conditions provide the opportunity to demonstrate the effect of stratification on height of sediment resuspension (section 3.5) and test if a steady, one dimensional model (section 3.6) can predict sediment resuspension height from measured velocity, wave, and stratification data.

\subsection{Background}

Large waves from winter storms are the main cause of sediment resuspension on the shelf and the strong currents associated with the same storms generate the highest suspended sediment flux (Cacchione et al., 1987; Wright et al., 1991; Cacchione et al., 1999), but half of suspended sediment transport on the inner shelf may happen during fair weather (measured: Wright et al., 1991; Sherwood et al., 1994; modeled: Styles and Glenn, 2005). While Traykovski et al. (1999) concluded that bedload dominates cross-shelf transport for medium to coarse grain sediments $(D 50=400 \mu \mathrm{m})$, the same may not be true for shelves with fine sand or mud, which suspend more easily. The inner shelf can exhibit periodic (Crockett and Nittrouer, 2004; Goff et al., 2005) or seasonal (Traykovski et al., 2007) mud deposits, which resuspend more easily than sand. The ease with which mud can be resuspended makes it more likely than fine sand to be affected by seasonal stratification and its long settling time makes its behavior more relevant than that of sand to applied environmental problems including pollutant dispersal and deposition, larval transport, and prediction of optical properties for naval and scientific marine operations.

On and off the shelf, sediment-induced stratification has been an active topic 
of research for many years (Adams and Weatherly, 1981; Glenn and Grant, 1987; Villaret and Trowbridge, 1991; Winterwerp, 2001) and in estuaries, stratification by temperature and salinity is a key factor in understanding sediment dynamics (Geyer, 1993; Scully and Friedrichs, 2003). However, the effect of temperature- and salinitybased stratification on the height of sediment resuspension on the continental shelf has been observed only occasionally (Souza et al., 2001; Chang et al., 2001; Glenn et al., 2008) and only on the mid-shelf during hurricane passage over a seasonal thermocline. As a result, the effect is sometimes neglected in models of sediment resuspension. For example, in their two-dimensional numerical model for cross-shelf transport of suspended sediment, Harris and Wiberg (2001) chose to omit effects of stratification in favor of the computational speed and simplicity from decoupled velocity and sediment calculations. The model is assumed to effectively represent most transport events on the shelf because temperature and salinity in the bottom boundary layer are usually well mixed during winter storms. However, even when stratification begins above a bottom boundary layer, it can still limit turbulence and reduce eddy diffusivity within the well-mixed lower part of the water column, as observed by Scully and Friedrichs (2003) in an estuarine context.

One recent project does provide particularly relevant results for this study. Glenn et al. (2008) found that in summer, the seasonal stratification limits midshelf sediment resuspension to below the pycnocline, even during hurricanes. Using optical backscatter from gliders, Glenn et al. observed three regimes of sediment resuspension during stratified, unstratified, and transitional storm events on the New Jersey shelf. In the mixed event, sediment was suspended throughout the water column while in the stratified event, it was limited by the thermocline that began $20 \mathrm{~m}$ below the surface in 40-50 m water depth. Temperature and optical backscatter profiles throughout the transitional event showed a clear progression from resuspension being capped by stratification to the thermocline being mixed away and backscatter becoming large and uniform over the entire water depth. Glenn et al.'s vertical profiles of optical backscatter ratio also show a transition from values of $w_{s} / u_{*}$, the ratio of 
sediment fall velocity to bottom shear velocity, in line with sediment suspension by wave-current interaction to values too high for bottom stress to be the sole source of turbulence, suggesting that surface wind mixing had penetrated throughout the water column to interact with the bottom boundary layer. In October 2005, Glenn et al. (2010) also observed a stratified and a mixed resuspension event south of Martha's Vineyard. These glider tracks passed over 25-45 m water depth at 20-70 km south of the island.

These optical glider observations are an excellent complement to our acoustic bottom-mounted ones and both types of observations provide long time series, enabling them to capture a wide range of conditions in a single deployment. The glider has the advantage of mobility, providing observations with high horizontal resolution. However, the horizontal and temporal progressions are linked and blur the distinction between spatial and temporal variability on the scales of inner shelf bed composition features. By contrast, the fixed acoustic measurements provide a better record of changes in time but, of course, are limited to specific deployment locations.

\subsection{Field Program}

The primary data set for this project is from the group of six NSF funded SWWIM (Stratification, Wind, and Waves on the Inner shelf of Martha's Vineyard) deployments south of Martha's Vineyard. The three and a half year data set is described in detail in Chapter 2 of this thesis. This chapter uses observations from three sites in $12,17.5$, and 27.5 meters water depth, located 1.5, 3.8, and 11.1 kilometers from shore, respectively. The 7 -m site did not have enough instances of concurrent stratification and waves to be significant in later analyses so will not be shown in this chapter. Moorings measured temperature and salinity throughout the water column while collocated upward-looking ADCPs provided velocity and acoustic backscatter measurements. We also use the continuously available data from the Martha's Vineyard Coastal Observatory node for wave statistics and Shore Mast and Air-Sea 
Interaction Tower (ASIT) for wind stress. Finally, three optical backscatter sensors provided data from one winter deployment at the 17-m site. Figure 2-1(a) shows the location of the field site on the US east coast and Figure 2-1(b) shows the instrument site locations relative to Martha's Vineyard.

\subsection{Data Processing}

\subsubsection{Backscatter intensity from received ADCP echo inten- sity}

In this Chapter, ADCP acoustic return is used to infer suspended sediment concentration. To relate the Received Signal Strength Indicator (RSSI) from an ADCP, $E$, to suspended sediment concentration, SSC, we compute an acoustic backscatter intensity variable, $S v$, that is representative of only the particles suspended in the water. We follow Gostiaux and van Haren's (2010) formulation of volume backscattering strength, $s_{v}$ as the appropriate form of the sonar equation derived by Urick (1983).

$$
s_{v}=\left(10^{\frac{K c E}{10}}-10^{\frac{K c E r}{10}}\right) \times R^{2} \times 10^{\frac{2 \alpha R}{10}} \times\left(\frac{T+273.16}{L \times P}\right) \times \text { Const } .
$$

(1)

(2)

(3)

(4)

(5)

(6)

In terms (1) and (2) of equation 3.1, $E$ is the ADCP received echo intensity and $\mathrm{Er}$ is error from the internal instrument noise floor. Both are recorded by the ADCP in counts. The single minimum value recorded by each beam during each deployment is used as Er, and this value comes from a time when the ADCP was out of the water at the beginning or end of each deployment. This value is substituted for the $E r$ value provided by a built-in test of the ADCP because the instruments were not available at the time of data processing. The $E r$ values range from 38 to 56, all near the example values of 40 - 43 given in the WorkHorse manual and below the limit for noise of 59 set by RDI. Errors within the range of the Er estimates would 
only skew the results at times of very low backscatter, which is unlikely to be when there is suspended sediment. Both $E$ and $E r$ are multiplied by $K c$, a beam-specific, manufacturer supplied value for the decibels/counts ratio. Since $K c * E$ and $K c * E r$ are in decibels, the subtraction must be done in log space $10^{(K c E / 10)}-10^{(K c E r / 10)}$.

Term (3) represents spherical beam spreading with $R$ equal to along-beam distance from the transducer to the center of the sample volume. Term (4) is sound absorption by water and sediment, with $\alpha=\alpha_{\text {water }}+\alpha_{\text {sed }}$ as the water and sediment absorption coefficient. $\alpha_{\text {water }}$ is calculated following Urick (1983). Following Hoitink and Hoekstra's (2005) implementation of Urick's (1948) formula for sound absorption by suspended sediment, $\alpha_{\text {sed }}$ is estimated for a ranges of particle sizes $(10-150 \mu \mathrm{m})$ and sediment concentrations $(1-1000 \mathrm{mg} / \mathrm{L})$ typical of the inner shelf. Absorption by sediment is estimated to be two to four orders of magnitude smaller than the sound absorption by water so $\alpha_{\text {sed }}$ is omitted in the calculation since the sediment concentration is unknown a priori.

Term (5) represents transducer power variation. $T$ is transducer temperature in Celsius, $L$ is transmit signal length in meters and $P$ is transmit power in Watts. The time-varying transmit power, $P$, is not known so the nominal values of $16 \mathrm{~W}$ for the $1200 \mathrm{kHz}$ ADCP and $37 \mathrm{~W}$ for the $600 \mathrm{kHz}$ ADCP given by the WorkHorse Technical Manual are used instead. There is one more unknown constant term (6) that includes instrument specific values that are time- and depth- independent such as transducer efficiency (Deines 1999, Eqn. A-8).

The volume backscattering strength, $s_{v}$, includes sound reflected by sediment as well as any other particles suspended in the sample volume so sediment concentration, SSC, is given by equation 3.2 where the total backscattering strength, $s_{v, \text { total }}$ is calculated from $s_{v}$ in equation 3.1, and $C 1$ and $C 2$ are empirical constants.

$$
\mathrm{SSC}=C_{1} s_{v, \text { total }}-C_{2} s_{v, \text { other }}
$$

If the water was perfectly clear, with no sediment or other acoustic reflectors, we 
would find $E=E r$ from equation 3.1 and $\mathrm{SSC}=0$ from equation 3.2. Backscatter strength often varies over many orders of magnitude, so it is common to use logarithmic units for backscatter intensity, $S v$, instead of linear ones.

$$
S v=10 \log _{10} s_{v}
$$

The conversion shown in equation 3.3 is mathematically sound so long as $E \neq E r$. Taking the $\log$ of both sides of equation 3.1 results in a logarithmic backscatter intensity, given by equation 3.4, where numbered terms are the same as equation 3.1.

$$
S v=10 \log _{10}\left(10^{\frac{K c E}{10}}-10^{\frac{K c E r}{10}}\right)+20 \log _{10} R+2 \alpha R+10 \log _{10}\left(\frac{T+273.16}{L \times P}\right)+C
$$

$(5)$

Constant $C$ now also includes the normalization constants to nondimensionalize the terms inside the logs. Because $C$ is instrument specific, we only compare absolute $S v$ values from a single ADCP in the following analyses while vertical variation in $S v$ can be compared between instruments. The terms are listed in order of importance in determining $S v$. The variation in size of the terms over the range of water depths, temperatures, and echo intensities are $60 \mathrm{~dB}$ for (1) and (2) combined, $30 \mathrm{~dB}$ for (3), $10 \mathrm{~dB}$ for (4) and $0.5 \mathrm{~dB}$ for (5)

For the 17, and 27-m sites, the backscatter intensity is calculated separately for each of the four ADCP beams and the median value is selected. The signals from the four beams all have correlation coefficients over 0.9 and regression slopes between 0.9 and 1.1 at the $95 \%$ confidence level (See Appendix 3.A), implying that the variation in sediment concentration is small over the scale of beam separation, which approaches $20 \mathrm{~m}$ near the surface above the deepest ADCP.

This $S v$ represents the total backscatter intensity, including the contribution from non-sediment scatterers in the water such as organic matter, zooplankton, bubbles, or any surface return signal reaching below the top $\sim 10 \%$ of the nominal water depth. Data from the top $10 \%$ of the water depth have a high return from surface reflection 
and are not used. As a first step towards isolating the backscatter contribution from sediment, we remove the noise level that is the minimum in-water value of computed $S v$. The results in section 3.5 and 3.6 are qualitatively unchanged from using the full $S v$ by removing the minimum value or the $1^{\text {st }}$ percentile value. Our backscatter variable becomes

$$
S v_{\text {new }}=10 \log _{10}\left(10^{\frac{\text { Sv,old }}{10}}-10^{\frac{\mathrm{min}(\text { Sv,old })}{10}}\right)
$$

In windy conditions, bubbles in the upper portion of the water column generate a backscatter intensity signal of similar order of magnitude to a large sediment resuspension event near the bed. $S v$ at $3 \mathrm{~m}$ below the surface and daily wind stress magnitude are well correlated ( $\mathrm{R}$ and $95 \%$ interval at 12, 17, 27-m sites are $0.52 \pm 0.04$, $0.84 \pm 0.02,0.82 \pm 0.02)$. Since waves and wind often occur together, it is sometimes hard to distinguish where the signal from sediment ends and that from bubbles begins. Since the only source of sediment is the sea floor, if we assume that lateral advection of sediment concentration gradients are small and that the system can adjust to a steady state in the time between our measurements (section 3.6.2), then it follows that the sediment concentration should never increase upwards. Above the height of a mid water column backscatter minimum, we do not expect the measured backscatter signal to primarily represent sediment.

\subsubsection{Optical backscatter calibration to suspended sediment concentration (SSC)}

Optical backscatter sensors were deployed for one wintertime SWWIM deployment and provide a second means of measuring suspended sediment concentrations. Optical backscatter is linearly proportional to suspended sediment concentration for any one particle size or for a fixed particle size distribution (D\&A Instrument Company, 2005; Lynch et al., 1994). However, the bed composition at this field site is highly variable in space and in time. Because of this variability, a near-bed reference concen-

tration formula (such as those explained in Cacchione et al., 2008) cannot be used to 
accurately predict the bed sediment availability at any particular time, so we cannot extrapolate upwards to predict the particle size distribution at the height of ADCP measurements at 2.75 mab and above. Still, calibration with bottom sediment is useful for several reasons. First, it demonstrates that the OBS used for this project do respond in a linear way to increasing concentrations of a fixed grain size distribution. Second, it provides a regression to compare measurements from different instruments on the same scale. Third, D\&A Instruments reports that their OBS sensitivity to particle size scales with $8.3 D^{-0.6}$, where $D$ is diameter (D\&A Instrument Company, 2005). Over the range of $16 \mu \mathrm{m}$ to $125 \mu \mathrm{m}$ diameters typical of this field site, the sediment concentration predicted from a particular OBS voltage response would only vary by a factor of four. If other brands of OBS behave similarly to the D\&A Instruments, then after calibrating with sediment that is a mixture of fine sand and mud, sediment concentration should be predicted from field measurements to within a factor of four.

In contrast to this commonly accepted principle of the optical backscatter to mass ratio varying with particle size, recent laboratory (Boss, personal communication, 4 May 2010) and field measurements (Boss et al., 2009; Hill, personal communication, 4 May 2010) suggest that for a fixed sediment grain population that is flocculated and unflocculated, the optical backscatter to SPM (suspended particle mass) ratio is much less variable than previously expected and relatively insensitive to aggregate size over the typical range of sizes. These results imply that optical instrument calibrations may not require the exact particle size distribution of the field measurements to be usefully accurate.

The five OBS deployed during SWWIM were later calibrated in a bucket using sediments grab-sampled from the bed of the 17-m site. Hill et al.'s (personal communication, 4 May 2010) optical and in-situ water samples suggest the suspended load near MVCO is primarily composed of mud flocs but the vigorous stirring needed to keep the sand fraction of our sediment samples suspended would not have allowed flocs to form during our bucket calibration. Calibration curves are shown in Appendix 
?? and their results are used qualitatively in section 3.6.

\subsubsection{Comparing Acoustic to Optical Backscatter}

Acoustic backscatter intensity scales linearly with the log of suspended sediment concentration, SSC, (Thorne et al., 1993; Thorne and Hanes, 2002) for a particular grain size or fixed grain size distribution. In this project, the SSC comes from calibrated optical backscatter and we temporarily ignore potential spatial and temporal variation in the suspended sediment grain size distribution that would vary this linear relationship. The moored OBS, described above in section 3.4.2, were used to calibrate the ADCP backscatter intensity. A regression of the three OBS deployed on the 17-m mooring against the nearest ADCP bins is shown in Appendix ??. The ADCP backscatter signal is correlated with the OBS signals, supporting the idea that the ADCPs are measuring sediment. Because we are not confident that the OBS calibration accurately represents field sediment concentration, we use the OBS-ADCP regression to confirm that the acoustic data is qualitatively reasonable, but do not further use the resulting regression coefficients.

\subsection{Observations}

The inner shelf south of Martha's Vineyard has strong semidiurnal tides, synoptic scale wind and wave forcing, and summertime stratification. The tides and winddriven circulation have been described in detail by Fewings et al. (2008) and wavedriven flow is described by Lentz et al. (2008). We summarize those results and elaborate on bathymetry, bed composition, wind, waves, surface heat flux, and stratification in Chapter 2 of this thesis.

Backscatter intensity and temperature measurements are used here to demonstrate the capping effect of stratification on sediment resuspension height. Time-series from two storm events with similar wind and wave conditions illustrate this phenomenon and mean profiles over various wave and stratification criteria confirm it. 
As described in section 3.4.1, the $S v$ calculation ignores an unknown, instrumentspecific constant. $S v$ values from different ADCPs cannot be compared directly, while variation in $S v$ in depth or time can be. Specifically, a single ADCP was used at each of the 17 and 27-m sites for SWWIM 2, 3, 5, 6, and 7. The ADCPs were accidentally swapped for SWWIM 4, so this deployment is omitted from calculations using absolute $S v$ in section 3.5.2. SWWIM4 is included in section 3.5.3 and subsequent sections that use $\Delta S v$, the value relative to the ADCP bin 2 value. The second ADCP bin is used as the reference level rather than the lowest because for all ADCPs the backscatter intensity at the lowest bin shows an unexplained and nonphysical decrease relative to the adjacent bin above. The error is visible at the bottom edge of the top two plots in Figure 3-1.

\subsubsection{Example of two synoptic events}

Two resuspension events from the $27-\mathrm{m}$ site in fall 2008 demonstrate both the effect of stratification on the height of sediment resuspension and some of the limitations of this data set. Figure 3-1 shows, on the left, an event in September that occurred during a time when the water was stratified and, on the right, an event in October when the water was well-mixed. These events are good examples because the wind and wave conditions were so similar. Both had $4 \mathrm{~m}$ waves with $7 \mathrm{~s}$ periods and strong wind stresses of $0.5 \mathrm{~Pa}$, which peaked earlier than the wave heights. Both events span 2.5 days. The top row shows acoustic backscatter from the ADCP. The strong surface signal from bubbles is apparent during both events but generally is separate from the sediment signal originating from the bottom. The second row of Figure 3-1 shows temperature. On the left the water is always stratified with $3{ }^{\circ} \mathrm{C}$ difference between top and bottom. Even during the peak wind stress when the upper portion of the water column becomes mixed, the bottom few meters of water remain several degrees cooler than the surface. On the right, the temperature is nearly uniform in depth and time. The variation is usually less than $0.03{ }^{\circ} \mathrm{C}$, which is roughly the limit of the instrument measurement accuracy. The black dots in both temperature panels 
show, if it exists, the height where the temperature reaches $0.1^{\circ} \mathrm{C}$ warmer than the bottom. The third and fourth rows of Figure 3-1 show significant wave height and wind stress magnitude.
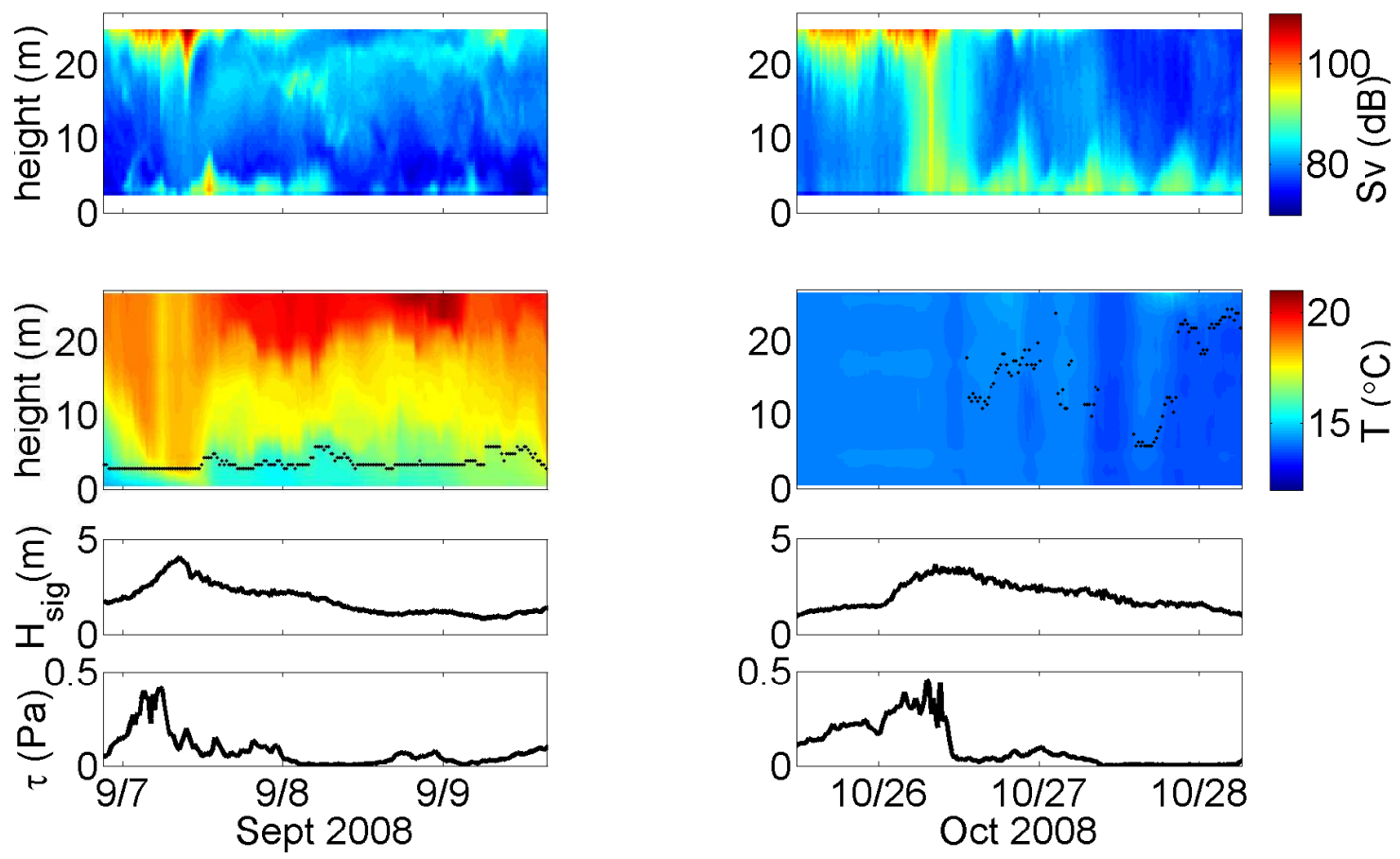

(a) Stratified event

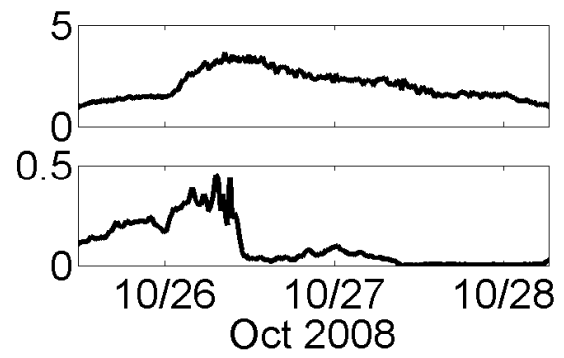

(b) Mixed event

Figure 3-1: Top to bottom, backscatter intensity, temperature and mixed layer thickness, significant wave height and wind stress magnitude for stratified (left) and unstratified (right) events in the fall of 2008

In both events, the wind preceded the waves, and a near-surface backscatter intensity signal from bubbles preceded the near-bed signal from sediment. At the peak of the stratified event on the left, the backscatter intensity drops an order of magnitude $(10 \mathrm{~dB})$ over the bottom seven meters of the water column. During the mixed event, on the right, the top of the first peak is obscured by surface signal, but at the second peak, the backscatter intensity signal takes $14 \mathrm{~m}$ to drop an order of magnitude. The difference between the heights of decay of the backscatter signals in these two events demonstrates that stratification can limit the height of sediment resuspension. The concurrence of waves with wind, which causes bubbles and a stronger surface 
reflections signal, does limit our ability to isolate the backscatter signal from sediment. The September event also provides an example of a signal we believe to be from zooplankton. On September 8, at around $15 \mathrm{~m}$ elevation, a patch of increased backscatter appears, apparently unconnected to any bottom or surface source. This signal persists around $10 \mathrm{~m}$ below the surface and is consistent with the behavior of zooplankton, as observed with acoustic backscatter by Baumgartner and Fratantoni (2008).

\subsubsection{Stratification reduces backscatter, independent of wave height}

Backscatter intensity throughout the water column tends to be lower in the summer than winter (Figure 3-2) and at least two factors contribute to this difference. The primary reason for the seasonal change is the tendency for smaller waves, which suspend less sediment, to occur in the summer and larger waves that suspend more sediment to occur in the winter. Another reason is that stratification tends to develop in the summer and it limits the mixing that moves sediment up away from the bed.

Figure 3-2 shows average summer (Apr - Sept) and winter (Oct - Mar) backscatter intensity profiles from the 12, 17, and 27-m sites. All three sites show lower average signal in summer than winter, with a $5 \mathrm{~dB}$ difference at the $17-\mathrm{m}$ site and a $2 \mathrm{~dB}$ difference at the 12 and 27-m sites. The larger difference between winter and summer at the 17-m site is notable and possibly an indication of the different sediment types on the sea floor, which will be discussed again later.

To separate the seasonal trend in wave energy from the effect of stratification on backscatter intensity, we compare backscatter profiles from stratified and unstratified times that are averaged over periods of similar wave heights. Figure 3-3 shows profiles of backscatter intensity from the 12,17 , and $27-\mathrm{m}$ sites. The horizontal axis is acoustic backscatter in decibels and the vertical axis is height above the bed. Dashed lines represent averages from times when there was stratification somewhere in the water 

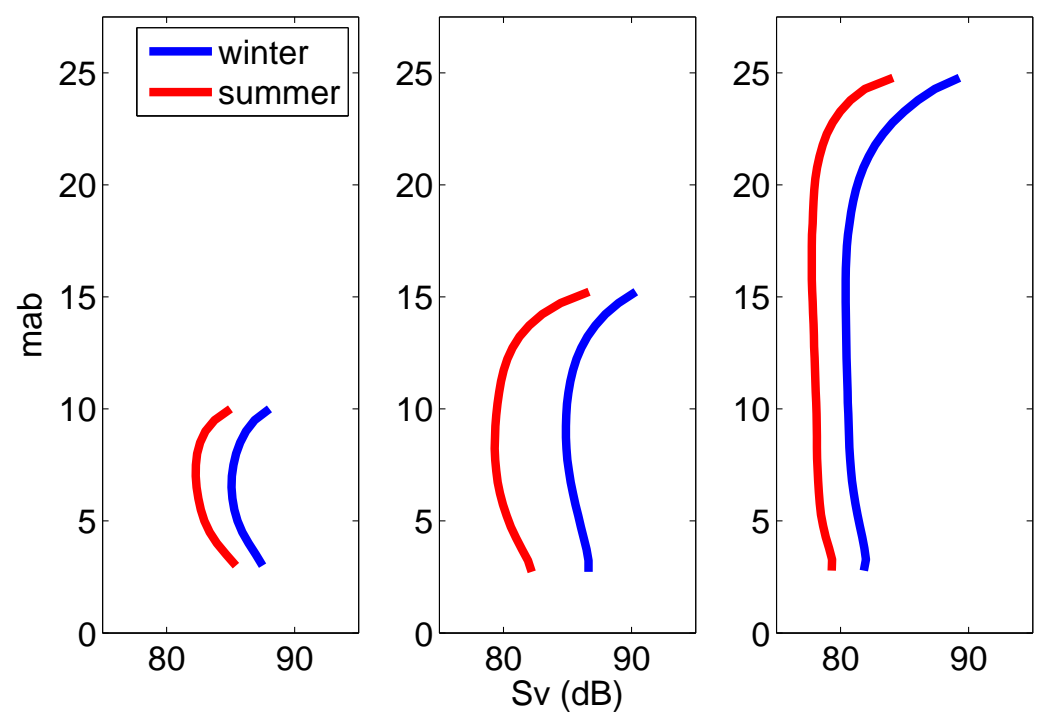

Figure 3-2: Average summer (red) and winter (blue) $S v$ profiles at 12, 17, and 27-m sites.

column and the solid lines are averages from times when the temperature was uniform. The criterion for stratification is an increase of $0.1{ }^{\circ} \mathrm{C}$ over $0.5 \mathrm{~m}$ increase in height occurring anywhere in the water column. Unstratified profiles have less than $0.5^{\circ} \mathrm{C}$ change over the entire water depth. Within each group of profiles, color represents wave height, ranging from less than half a meter up to greater than two meters significant wave height. To reduce the signal from bubbles at the surface, only times with wind stress magnitudes less than $0.03 \mathrm{~Pa}$ are included here. To ensure sediment was in suspension, backscatter intensity at 2.75 mab is required to be above the median value for that elevation.

Two important observations are evident in Figure 3-3. First, within each group, stratified or unstratified, the shape of the backscatter intensity profile does not change with wave height. For the unstratified conditions, the waves shift the overall magnitude up or down a little, but the shape stays the same. Variation with wave height between stratified profiles is not significant at the $95 \%$ confidence level. The fact that only magnitude, but not shape, changes with wave height is consistent with the theory that will be described in section 3.6, where a steady balance between sediment 


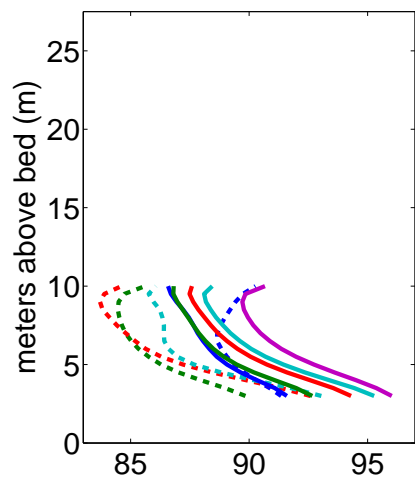

(a) $12 \mathrm{~m}$

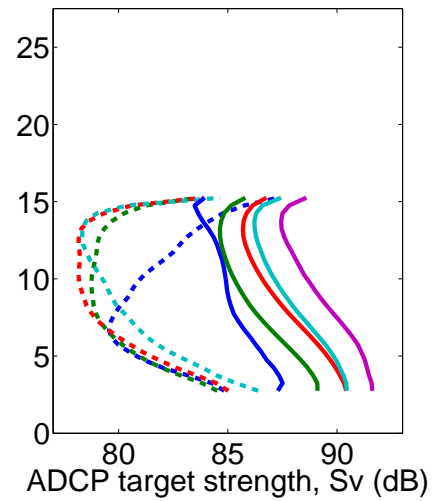

(b) $17 \mathrm{~m}$

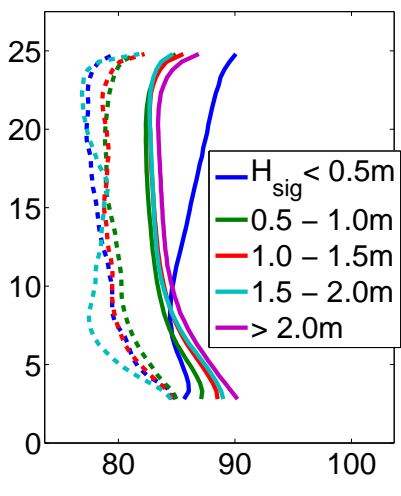

(c) $27 \mathrm{~m}$

Figure 3-3: Average backscatter intensity (dB) profiles for times when the water column was well-mixed (solid lines) or stratified (dashed). Color indicate wave height, from less than $0.5 \mathrm{~m}$ to more than $2.0 \mathrm{~m}$.

settling and turbulent mixing determine the shape of the concentration profile, while the magnitude is set by a bottom reference concentration that is partially based on wave shear stress.

Second, stratification causes an overall decrease in backscatter intensity and, in particular, a faster decrease in sediment with distance up, away from the bed. The combination of these two features means that the seasonal trend of seeing less resuspension in the summer when the water is stratified is not just the effect of smaller waves occurring in the summer, but actually an effect of the stratification. The stratified profiles from the 17-m site show a larger separation from the unstratified profiles than those at the 12 or $27-\mathrm{m}$ sites. One explanation is that the sediment available on the bed at the 17-m location is a finer particle size than at the other two locations and more susceptible to the limiting effect of stratification. This mud hypothesis could also possibly rationalize why the $17-\mathrm{m}$ site show the unexpected behavior of maintaining a large difference between stratified and unstratified profiles even at the lowest wave conditions. In the limit of no waves, we expect no resuspension, so there is not a reason to expect different backscatter values for stratified or unstratified nowave conditions. However, mud is very easy to resuspend and settles slowly so even times with waves less than $0.5 \mathrm{~m}$ shown by the blue lines in Figure 3-3, could have 
mud present in suspension.

\subsubsection{Height of resuspension follows mixed layer thickness}

Now that the effect of stratification reducing sediment resuspension has been estab-

lished, the bottom mixed layer thickness is compared to vertical structure of the backscatter intensity profiles. In other words, we compare the height above the bed where stratification begins to the height of sediment resuspension. Subtracting the second ADCP bin value from each profile collapses the variation in magnitude seen between wave heights in section 3.5.2. Then we can average together stratified profiles from all wave heights.

In Figure 3-4, profiles of temperature are in red, shown as differences from the bottom measurement. Profiles were selected for having specific mixed layer thicknesses, where the mixed layer is defined as having less than $0.1^{\circ} \mathrm{C}$ difference from the bottom temperature. Cutoff elevations depend on the heights of the deployed temperature sensors. For the $17-\mathrm{m}$ site in the middle panel, these thicknesses vary from 5 up to 13 mab, shown in the five panels from left to right. Mixed layer thicknesses of 4 to 10 mab are shown in four panels each for the 12 and $27-\mathrm{m}$ sites in the top and bottom row respectively. Only four panels are shown for the 27-m site because bottom mixed layer thicknesses above 10 mab did not provide statistically relevant data. Backscatter profiles averaged over the same groups of times are shown in blue. Like the temperature profiles, the backscatter profiles are plotted as the change in the signal compared to the lowest good bin in order to further collapse the slight variation with wave height seen in Figure 3-3.

For all three sites in Figure 3-4, the slope of the backscatter profiles as they decay away from the bed increases in the same order as the mixed layer thickness in the temperature profiles. The ever-present signal from bubbles or surface reflection in the upper part of the profiles should be ignored. This juxtaposition of temperature and backscatter intensity demonstrates that stratification not only reduces mid water column sediment concentrations (as shown in section 3.5.2), but also that as the 

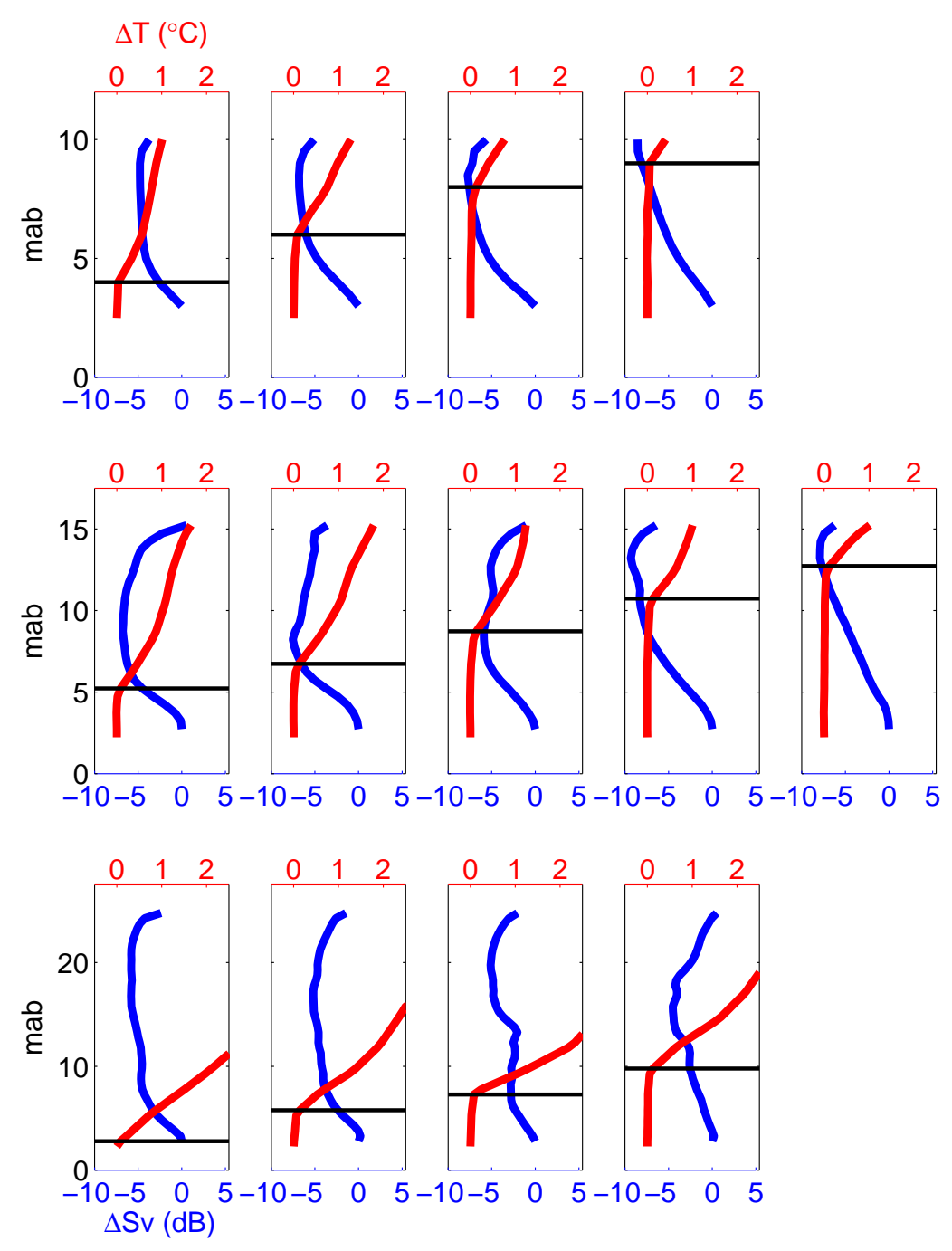

Figure 3-4: Backscatter intensity (blue) and temperature (red) variation for varying mixed layer thicknesses. Top, middle, and bottom rows shows 12, 17, and 27-m sites. Black lines indicate bottom mixed layer thickness

mixed layer thickness increases the backscatter signal from sediment reaches farther above the bed. For the 12 and 17-m sites, the backscatter reaches a minimum near elevation where the temperature stratification begins. When the stratification is only near the surface, like in the right-most panels for the 12 and 17-m sites, the profiles begin to look like the unstratified cases shown by the solid lines of Figure 3-3, since not much sediment gets up high enough to interact with the thermocline. 
Figure 3-5 shows the regression between mixed layer thickness and the height of the minimum $S v$ value in the averaged profiles (like those plotted in Figure 3-4) for the $17-\mathrm{m}$ site. The correlation is $R=0.88$ (95\% confidence interval $0.71-0.95)$ with a regression given by $y=(0.67 \pm 0.18) x+(4.0 \pm 1.7)$. The correlation between mixed layer thickness and the height of the minimum $S v$ value is not significant at the 12 or 27-m sites; this is unsurprising at the 27-m site because the profiles in Figure 3-4 do not appear to reach a minimum at the height of the mixed layer thickness, but rather continue to decrease for 5 to 10 more meters. The $27-\mathrm{m}$ profiles also appear less smooth than the 12 or 17-m ones and may be dominated by patches of suspected zooplankton in the mid and upper water column. Both the 12 and 27-m sites provide fewer instances of sediment meeting the bottom of the thermocline than the $17-\mathrm{m}$ site. At the 12-m site, the shallower depth means the mixing effect of wind and waves extend through a larger fraction of the water column than at the 17-m site, providing fewer times with strong stratification. At the 27-m site, the greater depth means the surface-originated stratification often does not reach deep enough to interact with suspended sediment.

\subsection{Theory and models for sediment suspension}

Now that we have a measure of suspended sediment in the water column, we compare it to predictions by traditional theory based on the other environmental variables: currents, waves, stratification, and wind stress. We ask whether simplified models are robust enough to describe sediment suspension in decidedly non-idealized field conditions where we know several of the necessary assumptions for the theory are, in reality, violated.

\subsubsection{Bed shear velocity and particle size}

For a given bottom roughness and particle size, a critical level of shear stress, $\tau^{b}$, or

shear velocity, $u_{*}$, where $u_{*}^{2} \equiv \tau^{b} / \rho$, must be surpassed to initiate resuspension of 


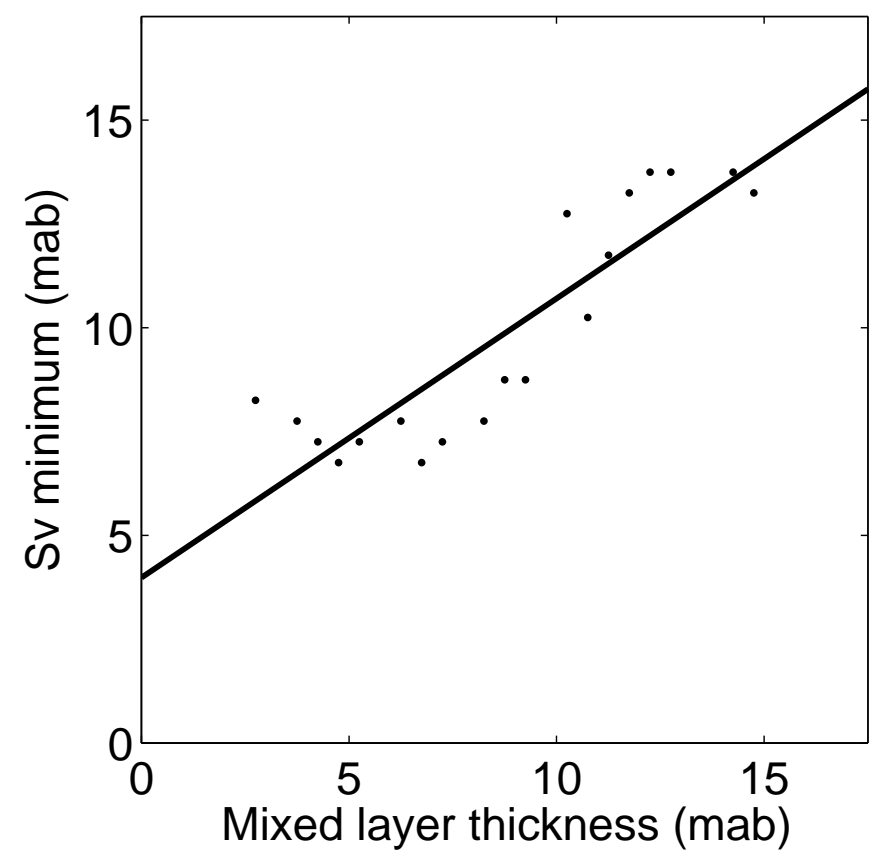

Figure 3-5: Mixed layer thickness vs. height of minimum $S v$ in averaged profiles (.) with correlation coefficient $\mathrm{R}=0.88(95 \%$ : $0.71-0.95)$. Line given by $y=$ $(0.67 \pm 0.18) x+(4.0 \pm 1.7)$

sediment from the bed. Smaller bottom roughness and larger particle size increase the critical shear. Both mean currents and wave orbital velocities contribute to the bottom shear velocity, but their relative importance varies with water depth.

We expect the particle sizes measured by the ADCP backscatter to be somewhat less variable than the bed composition described in section 2.2.1 due to resuspension criteria and the selection of forcing conditions presented here. A shear velocity of 1.3 $\mathrm{cm} / \mathrm{s}$ is required to initiate suspension of fine sand from a sandy patch or mud from a mud patch based on the modified Shields parameter (Smith, 1977) for critical shear stress. A shear velocity of just $0.4 \mathrm{~cm} / \mathrm{s}$ is needed to lift the finer mud off a rougher sandy patch.

We use Madsen's (1994) wave-current boundary layer model and a quadratic bottom drag coefficient, $C_{D}$, of $1.45 \times 10^{-3}$ (following Fewings (2007) value from J. Trowbridge) to calculate wave-, current-, and combined wave-current shear velocities from measured waves and currents. At $12 \mathrm{~m}$, the current shear velocity is larger than 
the wave shear velocity $5 \%$ of the time. At $17 \mathrm{~m}$, the current shear velocity is larger than the wave shear velocity $19 \%$ of the time. Finally, at $27 \mathrm{~m}$, the current shear velocity is larger a majority, $57 \%$, of the time. Table 3.1 summarizes the fraction of time the combined wave-current shear velocity exceeds the resuspension criteria for sand and mud and the correlation coefficient between the shear velocity and $S v$ at the second ADCP bin.

Table 3.1: Fractions of time mud and sand are expected to be suspended, and correlation coefficient between shear velocity, $u_{*}$, and backscatter intensity, $S v$

\begin{tabular}{llll}
\hline Site & $\%$ time $u_{*}>u_{* \text { mud }}$ & $\%$ time $u_{*}>u_{* \text { sand }}$ & $R(>99 \%$ level $)$ between $S v$ and $u_{*}$ \\
\hline \hline $12-\mathrm{m}$ & $100(99.70)$ & 76 & 0.54 \\
$17-\mathrm{m}$ & 94 & 42 & 0.46 \\
$27-\mathrm{m}$ & 75 & 13 & 0.59 \\
\hline
\end{tabular}

Calculated wave-current shear velocities describe only $29 \%, 21 \%$, and $34 \%$ of the observed variance in backscatter intensity from the lowest ADCP measurements at the 12,17 , and $27-\mathrm{m}$ sites. The presence of mud in the backscatter measurements is likely limited by patchy bed availability, though these correlation values do not increase if the calculation is restricted to times when shear velocity estimates predicts sand to be resuspended. While the bottom shear stress often exceeds the resuspension criteria for fine sand, only the larger storms should lift it throughout the water column. It would not be surprising to see fine sand concentrations of tens of milligrams per liter at 1.2 mab, like those observed on the Hudson shelf by Harris et al. (2003), or a gram per liter at 0.15 mab like Storlazzi and Jaffe (2002) on the central CA shelf, but large waves are usually accompanied by large wind, so many big events have been omitted from this analysis. For those remaining events, recall that the concentration is still decaying upward and the lowest backscatter is at 2.75 mab so incipient fine sand suspension does not imply it will reach the ADCP sample volumes.

A more compelling reason not to expect sand to have reached the ADCP sampling volume comes from measurements made during the OASIS program (see section 2.1.4). Hill et al. (personal communication, 4 May 2010) measured flocculated particles ranging from tens to hundred of microns in diameter with distributed but con- 
sistent settling velocities of around $1 \mathrm{~mm} / \mathrm{s}$, but reported that very few sand grains reached 1.2 mab during the (typical) autumn conditions of that experiment. With our lowest ADCP bin higher above the bed and in deeper water than the OASIS in situ water sampler, even less sand should reach our sampling volume for similar wave conditions.

Finally, we are convinced the measured sediment is typically flocculated mud by the comparison to theory that follows in section 3.6.3, which demonstrates that the backscatter signal is severely under- and over-predicted by estimates made with fine sand and disaggregated mud respectively. In the following section, we show that using the canonical mud-floc settling velocity of $1 \mathrm{~mm} / \mathrm{s}$ provides a much better match to the measurements.

\subsubsection{Sediment concentration predictions from an eddy vis- cosity profile}

Sediment concentration, $c_{s}$, profiles are derived following the standard model of a Rouse $(1937,1961)$ profile. This model is based on a steady balance between particles falling under gravity and being lifted by turbulent mixing. The settling velocity, $w_{s}$, is the vertical velocity a sediment particle would have in still water. It is determined by particle size and density, and is positive downward by convention. We assume the eddy diffusivity for sediment, $K_{c}$, is equal to the eddy viscosity, $K_{m}$. By Reynolds averaging, the conservation of mass equation for suspended sediment reduces to

$$
-w_{s} c_{s}=K_{m} \frac{\partial c_{s}}{\partial z}
$$

This equation can be integrated in $z$ to solve for sediment concentration as a function of height. The solution will depend on the concentration given at some reference level. In section 3.6.3, measured velocity and density profiles are used to estimate the eddy viscosity, which is then used to numerically integrate the sediment mass equation. The reference level becomes $2.75 \mathrm{mab}$, the height of the lowest ADCP 
measurements, and all estimated concentration profiles are relative to the (unknown) concentration at 2.75 mab.

Sections 3.6.4 and 3.6.5 compare the observed backscatter profiles to suspended sediment predictions from analytic models for eddy viscosity. The unstratified case is used first to consider under what conditions an established turbulence closure scheme matches acoustic and optical observations when the water is well mixed. Then, observed stratification is included in sediment predictions that are compared to acoustic backscatter measurements during stratified conditions.

\subsubsection{Using Mellor-Yamada 2.0}

We test if an established turbulence closure scheme and a 1D model (equation 3.6) can predict sediment resuspension height from measured velocity, wave, and density data. Weatherly and Martin's (1978) implementation of the Mellor and Yamada (1974) level 2.0 turbulence closure scheme is used to generate eddy viscosity profiles. The 2.0 scheme is appropriate here because the profiles averaged together in this analysis are selected based on the hydrodynamic conditions at each measurement time and so are not sequential in time. Without sequential time steps, the MellorYamada 2.5 scheme that diffuses momentum cannot be used, but with the steady state assumption for sediment we also assume the flow has naturally adjusted to any momentum diffusion and that measured profiles of velocity and temperature fully constrain the turbulence. The gradient Richardson number, $R i=N^{2} /(\partial u / \partial z)^{2}$, represents the ratio of reduction of turbulence by stratification to production by shear and Mellor-Yamada 2.0 uses $R i$ in computing the turbulent length scale. At small levels of shear and stratification, $R i$ becomes very sensitive to small fluctuations in both values so temperature and velocity profiles are smoothed with a running 3-meter average before being used in the Mellor-Yamada model.

One addition has been made to Mellor and Yamada's turbulent length scale computation. Their description is for only a bottom boundary layer with the assumption that $z$ goes to infinity far from the boundary. On the inner shelf, the bottom and 
surface boundary layers overlap and distance to both can limit the turbulent length scale so Wijesekera et al.'s (2003) effective distance from boundary is used in place of the height above bed in Mellor and Yamadas formula. In the Mellor and Yamada (1974) length scale,

$$
l=\frac{\kappa z}{1+\frac{\kappa z}{l_{0}}}
$$

we replace $z$ with Wijesekera et al.'s $z_{\text {effective }}$, given by

$$
\frac{1}{z_{e f f}}=\frac{1}{z}+\frac{1}{H-z}
$$

The Mellor-Yamada 2.0 closure scheme is implemented with inputs of measured velocity and density profiles as well as guesses at eddy viscosity and turbulent kinetic energy. Eddy viscosity, diffusivity, turbulent kinetic energy and turbulent length scale are returned. The calculation is iterated until the solution converges (usually just two iterations). Using the eddy viscosity profile generated with this closure scheme, sediment concentration profiles are calculated for three sediment types: fine sand with fall velocity, $w_{s}=10 \mathrm{~mm} / \mathrm{s}$, mud flocs at $w_{s}=1 \mathrm{~mm} / \mathrm{s}$, and individual mud grains at $w_{s}=0.02 \mathrm{~mm} / \mathrm{s}$. These estimates were made with velocity and temperature data from all deployments at the 12, 17, and 27-m sites.

\subsubsection{Comparison to Mellor-Yamada 2.0 for unstratified con- ditions}

ADCP backscatter at a single acoustic frequency does not provide any information about particle size. We test our working hypothesis about the particle size by comparing the measured profiles to those predicted for various fall velocities, which are associated with particular particle types. The predictions for three particle sizes, plus ADCP and OBS measurements, are shown in Figure 3-6. The thick teal line is the measured ADCP backscatter, black circles are the OBS measurements, and red, green, and blue lines are concentration predictions for fine sand, mud flocs, and mud 
grains, respectively. All five lines are averages over unstratified conditions from the one winter deployment when OBS data are available. The three predictions and the ADCP data are shown as change relative to the value at 2.75 mab. The lowest OBS was located at 5 mab, so the OBS value at 5 mab is set to match the ADCP value at that height and the upper OBS measurements are shown relative to the value at 5 mab. These profiles are limited to times when wind stress is small and the lowest $\mathrm{ADCP}$ bin returns a value $2 \mathrm{~dB}$ higher than the seasonal trend.

A fall velocity of $1 \mathrm{~mm} / \mathrm{s}$ produces a concentration profile that is similar to the ADCP and OBS measurements. This fall velocity is typical of the mud flocs observed with optical and physical sampling at a nearby field site during the separate but simultaneous OASIS experiment.

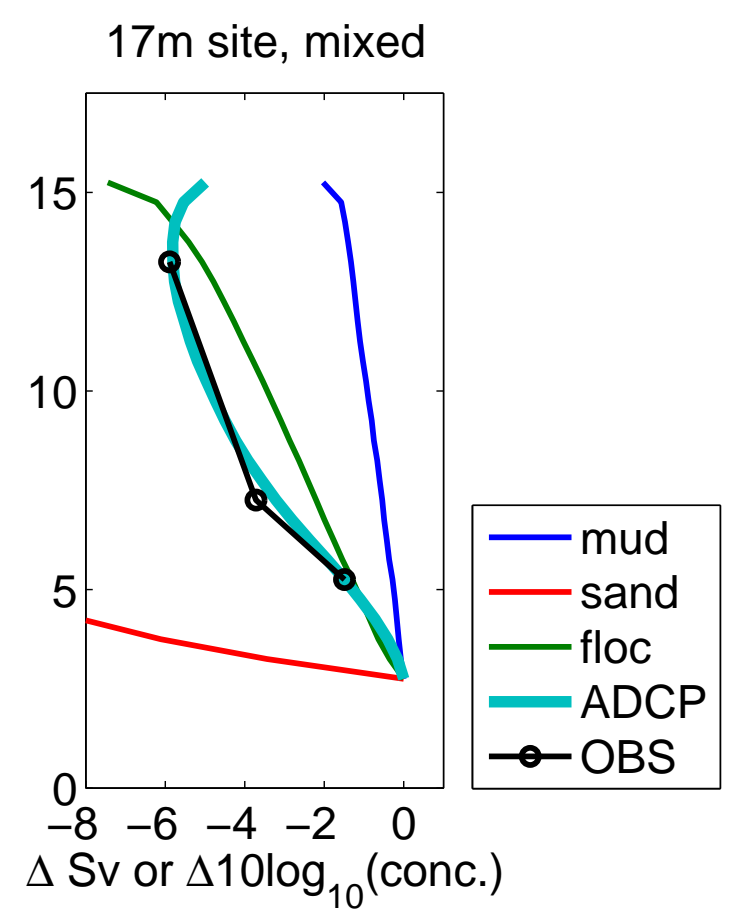

Figure 3-6: ADCP and OBS data compared to Mellor-Yamada 2.0 model prediction for unstratified times when the OBS were deployed (SWWIM 6, 17-m site)

The OBS were only deployed at one site for one season, but Mellor-Yamada 2.0 predictions can be compared with ADCP measurements for the entire data set at 12, 17, and 27-m sites. Figure 3-7 shows predicted and ADCP profiles averaged over 
unstratified conditions for all deployments. The three sites and full data set further support the expectation that mud flocs are the main sediment type being suspended around the Martha's Vineyard inner shelf.

In Figures 3-6 and 3-7, the $1 \mathrm{~mm} / \mathrm{s}$ mud floc fall velocity has the closest slope to that of the ADCP measurements. We note, however, that the curvature of the ADCP profiles is not replicated by any of the three predicted concentration profiles. The difference may be due to the presence of bubbles. Bubble concentration is highest at the surface and decays downwards. When summed with the sediment concentration, which decays upwards, the bubbles will cause a curvature to the ADCP backscatter profile that is qualitatively similar to the observed difference in curvature between the ADCP backscatter and predicted mud floc profiles. Without the enhanced backscatter from bubbles, the ADCP value would be lower, so the mud floc fall velocity use here may be slightly slower than the average value for the sediment in the field.

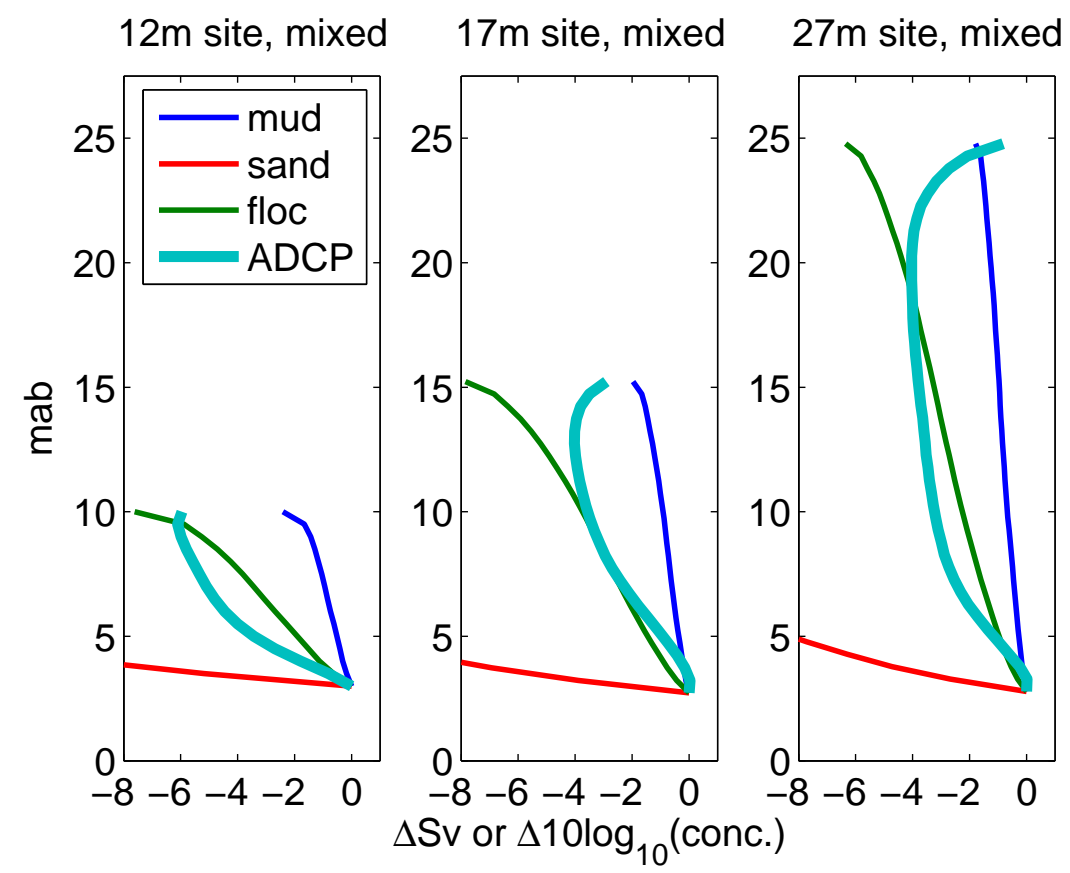

Figure 3-7: ADCP backscatter measurements and Mellor-Yamada 2.0 model prediction for unstratified conditions over all SWWIM deployments at the 12, 17, and 27-m sites 


\subsubsection{Comparison to M-Y 2.0 for stratified conditions}

Temperature and acoustic backscatter data show a clear relationship between mixed layer thickness and the height of sediment resuspension (section 3.5) and the MellorYamada 2.0 turbulence closure scheme combined with flocculated mud properties proved a reasonable match to the ADCP backscatter profiles in unstratified conditions (section 3.6.4). Now, we test if the model can also reproduce the backscatter measurements at times when there was stratification above various mixed layer thicknesses. Figure 3-8 shows ADCP backscatter and predicted sediment concentration profiles for five mixed layer thicknesses at the 17-m site (top row) and four mixed layer thicknesses for each of the 12 and 27-m sites (bottom row). The colors represent the same data types as in Figure 3-7.

When a clear distinction between mud and flocculated mud concentration predictions is visible, a fall velocity of large mud flocs (green line) again best matches the observations (thick, teal line). The variability in the spacing between the measurements and floc concentration predictions likely represent the variability in the properties of the available bed sediment. The model predicts a sharp drop in sediment concentration at the top of the mixed layer and this decrease in concentration is much sharper in the predictions than in the ADCP observations. There are several possible reasons for this discrepancy. Bubbles or surface signal raise the ADCP signal in the upper part of the water column, but are not in the model prediction. This closure scheme may not fully capture the nature of the turbulence at the top of the mixed layer. Other physical phenomena that have been ignored in the sediment profile computation may be relevant, including the non-steady and three-dimensional nature of the real sediment mass conservation balance. For the 27 -m site, the backscatter profiles were not particularly well represented by the corresponding temperature profiles (section 3.5.3) so it is not surprising that they also are not similar to predicted profiles based on the same temperature data. 


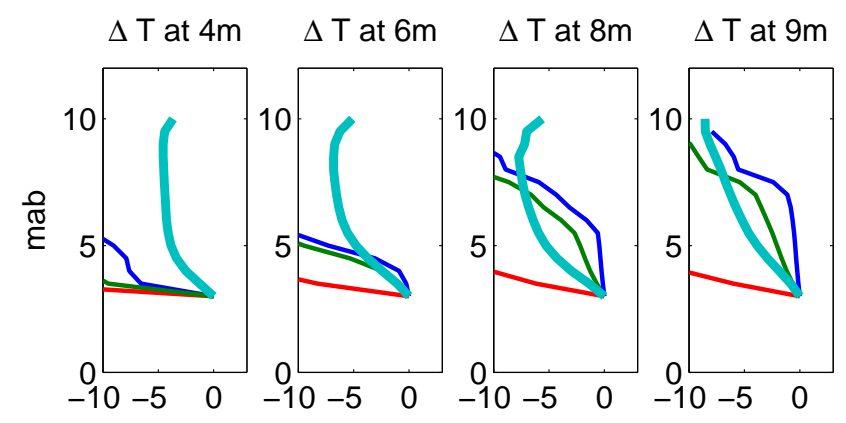

$\Delta \mathrm{T}$ at $5.23 \mathrm{~m} \Delta \mathrm{T}$ at $6.73 \mathrm{~m} \Delta \mathrm{T}$ at $8.73 \mathrm{~m} \Delta \mathrm{T}$ at $10.73 \mathrm{~m} \Delta \mathrm{T}$ at $12.73 \mathrm{~m}$

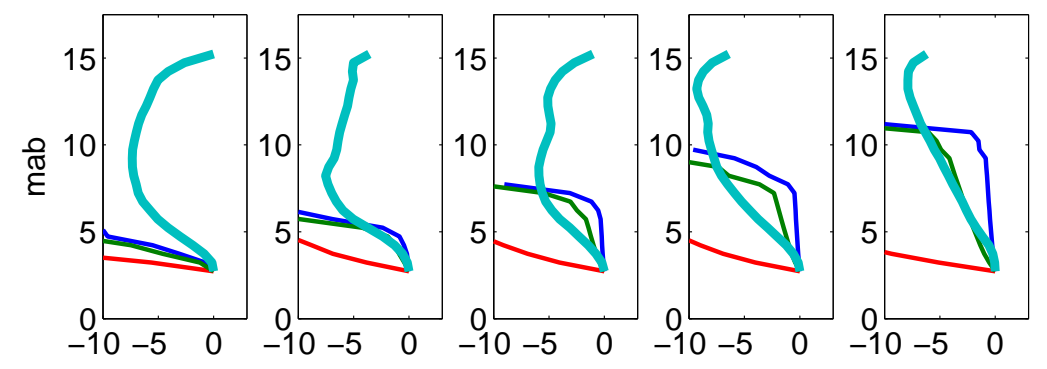

$\Delta \mathrm{T}$ at $2.78 \mathrm{~m} \Delta \mathrm{T}$ at $5.78 \mathrm{~m} \Delta \mathrm{T}$ at $7.28 \mathrm{~m} \Delta \mathrm{T}$ at $9.78 \mathrm{~m}$

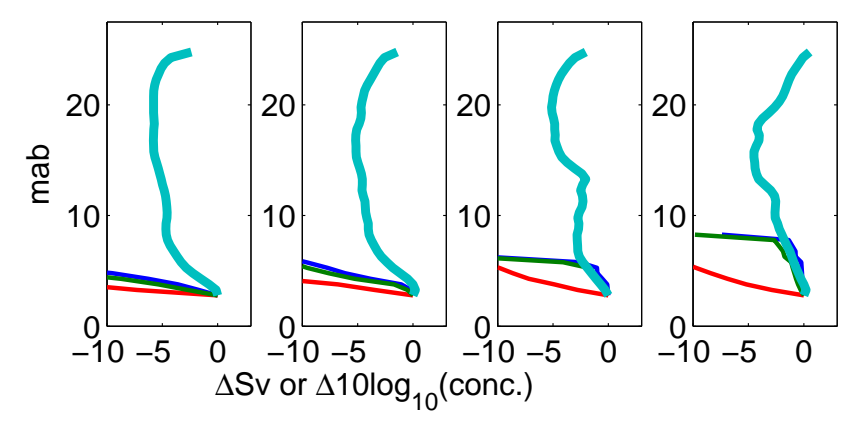

Figure 3-8: ADCP backscatter and sediment concentration predictions for various mixed layer thicknesses at 12,17 , and 27 -m sites

\subsection{Implications for Transport}

\subsubsection{Influence of changes in the sediment concentration pro- file}

Sediment transport depends on both the sediment concentration and the velocity of the water that moves it. Stratification will affect the vertical structure of both of these 
variables but in this section we neglect the effect of stratification on the circulation in order to isolate the influence of the change in the sediment concentration profile on the sediment transport. Without calibration to actual sediment concentrations, we cannot discuss sediment transport in terms of kilograms or cubic meters of material, but we can use the model described in section 3.6 to address the effect of mixed layer thickness on total suspended load and transport. Here, we use idealized profiles of velocity and temperature that are representative of our field conditions to make predictions for eddy viscosity using the Mellor-Yamada 2.0 closure scheme. Those eddy viscosity profiles are integrated to make predictions for the profiles of sediment concentration relative to the near-bed value. This example uses a single velocity profile in combination with three different temperature profiles to compare sediment concentration predictions for the three levels of stratification. A depth of $17.5 \mathrm{~m}$ is chosen to match the depth at our 17-m field site. We consider the cases of an unlimited sediment supply and one that is limited by the total volume available for resuspension from the bed.
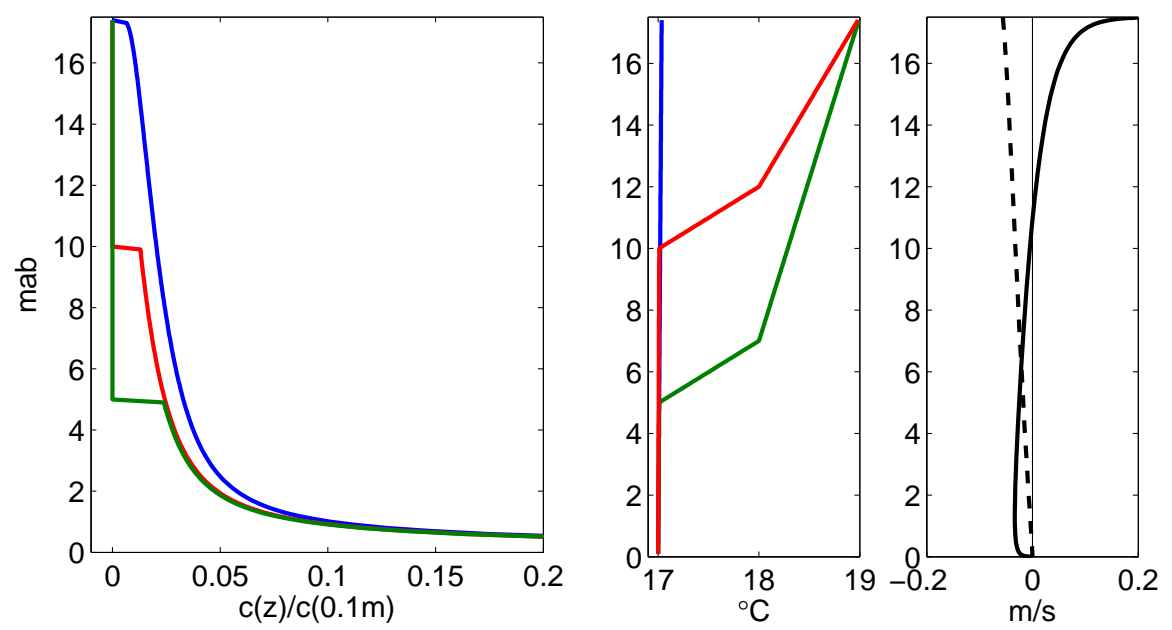

Figure 3-9: Left: sediment concentration as fraction of value at 0.1 mab for mixed (blue) and stratified (red) conditions with mixed later thicknesses of 5 and $10 \mathrm{~m}$. Center: temperature profiles for mixed (blue) and stratified (red) conditions. Right: velocities, $u$ (thick solid) and $v$ (dashed).

The velocity profile is generated by a simple numerical model that solves the one 
dimensional (vertical), steady, shallow water equations and uses the assumption of no net cross-shelf transport to estimate the cross-shelf pressure gradient. The model is described in detail in Lentz (1995), and Fewings et al. (2008) demonstrated that it is a good predictor of wind-driven inner shelf circulation in unstratified conditions. The eddy viscosity used here is a cubic profile with linear slopes to zero magnitude at both boundaries. The total amplitude is divided by $\sqrt{2}$ to crudely approximate a Mellor-Yamada 2.5 closure scheme for unstratified conditions. The model matches surface and bottom boundary conditions given by a prescribed surface wind stress and effective bottom roughness. The bottom roughness measured near MVCO that was used in section $3.6\left(C_{D}=1.45 \times 10^{-3}\right)$ is used again here. An offshore wind stress of $0.1 \mathrm{~Pa}$ is used to force the model. Velocity profiles of $u$ (solid) positive offshore and $v$ (dashed) alongshore are shown in the right panel of Figure 3-9. The thin vertical line indicates zero.

The temperature profiles, shown in the center panel of Figure 3-9, are made to mimic those measured profiles in Figure 3-4. The three profiles represent a mixed water column (blue) and bottom mixed later thicknesses of 5 (green) and $10 \mathrm{~m}$ (red). All profiles are $17^{\circ} \mathrm{C}$ at the bed. The two profiles with stratification each warm by one degree over the two meters at the top the the mixed later and a second degree over the remaining water column height to the surface.

The velocity and temperature profiles are used to generate an eddy viscosity profile from the Mellor-Yamada 2.0 closure scheme. The eddy viscosity profile can be used to predict vertical changes in sediment concentration by rearranging equation 3.6 and integrating in $z$. The right hand side of equation $3.9 \mathrm{~b}$ is numerically integrated upwards from a typical wave-current boundary layer thickness of $z_{o}=0.1 \mathrm{~m}$ and the result applied to equation $3.9 \mathrm{c}$ to predict the relative sediment concentrations at each height above 0.1 mab. 


$$
\begin{aligned}
\int_{z_{o}}^{z} \frac{\partial c / \partial z}{c} d z & =\int_{z_{o}}^{z} \frac{-w_{s}}{K_{m}} d z \\
\ln \left(\frac{c}{c\left(z_{o}\right)}\right) & =-w_{s} \int_{z_{o}}^{z} \frac{1}{K_{m}} d z \\
\frac{c(z)}{c\left(z_{o}\right)} & =\exp \left(-w_{s} \int_{z_{o}}^{z} \frac{1}{K_{m}} d z\right)
\end{aligned}
$$

The left panel of Figure 3-9 shows the resulting sediment concentration predictions, plotted as a fraction of the concentration at 0.1 mab. In stratified conditions (red and green lines), suspended sediment load is significantly reduced compared to well-mixed conditions (blue). By summing the concentration curves in Figure 3-9 from $0.1 \mathrm{mab}$ to the surface, we estimate the suspended sediment load for 5 and 10 m mixed later thicknesses to be $59 \%$ and $73 \%$ of the value for well-mixed conditions given the same concentration at 0.1 mab. This large reduction in suspended load predominantly comes from above the height of the mixed layer thickness so an additional effect on the net transport of sediment will depend on the shear in the velocity profiles.

For the velocity profiles used here, $v$ is always negative and increases upwards so the along-shelf transport will be reduced in stratified conditions, but the direction will be the same. 5 and $10 \mathrm{~m}$ mixed layers reduce the along-shelf sediment transport to 18 and $40 \%$ of the value for mixed conditions. The $u$ profile crosses from on- to offshore around 11 mab so any sediment suspended above this height will be transported in the opposite cross-shelf direction from sediment lower in the water column. In this case, all three scenarios have net onshore transport with a $5 \mathrm{~m}$ mixed layer generating $90 \%$ of the transport compared to the mixed case. The 10 m layer causes an increase in net (onshore) transport of $2 \%$ over the mixed case because the concentration drops off near where the flow reverses. In the mixed case, the offshore transport near the 
surface balances some of the onshore transport lower in the water.

If instead of assuming that all three conditions for stratification would have the same reference concentration at $z=0.1 \mathrm{~m}$, we assume that the same total volume of sediment is suspended, but distributed differently throughout the water column, we can normalize the concentration profiles from Figure 3-9 to make new profiles, shown on the left side of Figure 3-10. By forcing the area under the three concentration curves to be the same, the profile with the sediment trapped closest to the bed (green), has the highest reference concentration, nearly twice the reference concentration for the unstratified curve (blue).
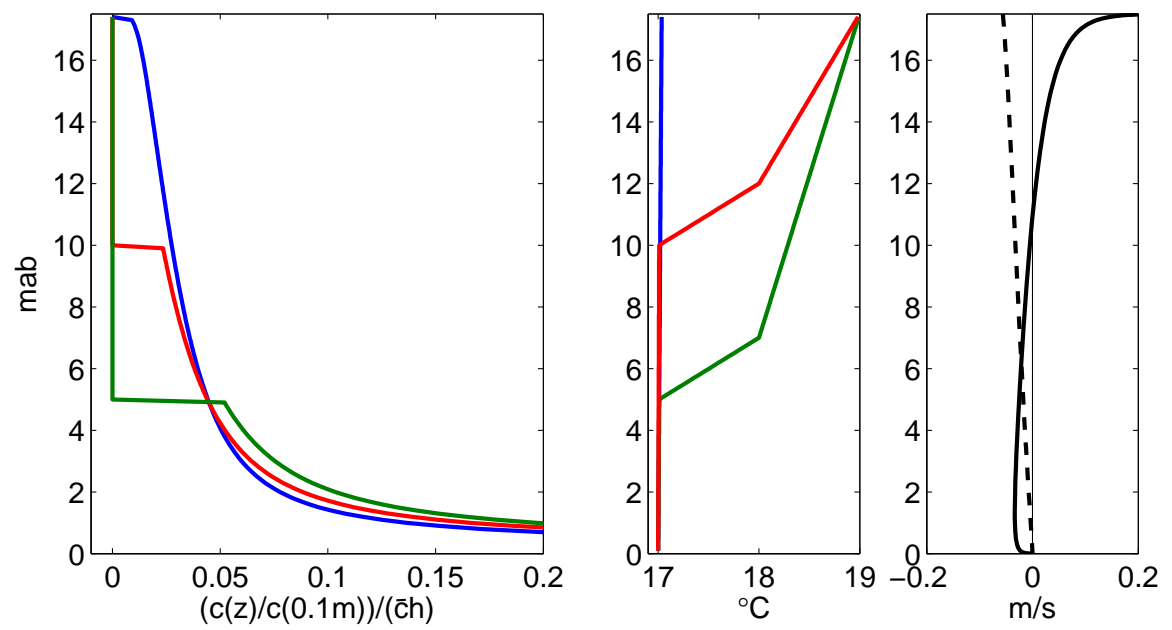

Figure 3-10: Left: Sediment concentration as fraction of value at 0.1 mab, normalized to have the same total sediment volume for mixed (blue) and stratified (red) conditions with mixed later thicknesses of 5 and $10 \mathrm{~m}$. Center: temperature profiles for mixed (blue) and stratified (red) conditions. Right: velocities, $u$ (thick solid) and $v$ (dashed).

When we estimate transport from the velocity profiles with these normalized concentration profiles, we again find a decreased magnitude of along-shelf transport for thinner bottom mixed layers. The 5 and $10 \mathrm{~m}$ mixed layers create 29 and $54 \%$ of the along-shelf transport as the unstratified profile. These fractions are larger than for the constant reference concentration method because now the stratified profiles have a higher concentration than the mixed case in the lowest $5 \mathrm{~m}$ of the water column. 
For the cross-shelf transport, again all three scenarios generate net onshore transport by the offshore wind stress because the highest concentrations are in the return flow in the lower portion of the water column. However, now the presence of sediment in the upper portion of the water column for the unstratified case necessarily means it also has a lower concentration of sediment in the lower portion of the water column compared to the two stratified cases. The mixed case drives the smallest magnitude cross-shelf transport and the 5 and $10 \mathrm{~m}$ mixed layer cases have 46 and $27 \%$ more transport, respectively. This second method of estimating normalized concentration profiles may be more applicable than the first method for cases where the the sediment supply is limited, like the patchy mud found south of Martha's Vineyard.

The transport fractions reported here are, of course, specific to the velocity profiles and mixed layer thicknesses selected, but this example demonstrates that stratification far from the bed still has an effect on net transport. In this section, the velocity profiles were not adjusted in response to the stratification. Vertical gradients of velocity are small in the middle of the water column compared to near the surface or bottom but the presences of stratification does increase the shear in the velocity profile (Fewings et al., 2008; Lentz et al., 2008; Chapter 4 of this thesis). An increase in shear around the height where sediment concentration drops suddenly should enhance the effect of stratification on transport. This topic is discussed in the following section, in combination with observational results from Chapter 4, which detail the effect of stratification on wind-driven cross-shelf circulation.

\subsubsection{Influence of changes in the velocity profile}

This section discusses the implications of and limitations on applying our understanding of stratified circulation to sediment transport estimates. In section 3.7.1, we discussed the effect of stratification capping sediment resuspension height on sediment transport without considering how stratification simultaneously affects the circulation. In observations in Chapter 4, stratification increases cross-shelf transport in the surface boundary layer by roughly two times for cross-shelf wind (Figure 4-17) and 
10 times for along-shelf wind (Figure 4-11). At first pass, these increases in transport seem much larger than the 30-40\% increase caused by the capping effect. However, even though equal and opposite water mass transport occurs in both boundary layers, the distribution is not symmetric around the mid water depth. In the surface boundary layer, the largest increase in velocity is at the surface. In the return flow, the increase with stratification is distributed over the lower portion of the water column, and the velocity still goes to zero at the bottom boundary. Because the highest sediment concentrations are very near the bed, the transport calculation is sensitive to the near bed velocities, which occur far below the $2.75 \mathrm{~m}$ height of our lowest velocity measurement. This gap in the measurements makes the sediment transport calculation very sensitive to the method we choose to extrapolate the velocity measurements to the bed in a way that the water mass transport calculations were not.

For cross-shelf velocities driven by along-shelf winds, such as those shown in Figure 4-6, it is clear that the velocities in the stratified cases will be larger magnitude than those for unstratified cases all the way to the bed. We can comfortably estimate that the suspended sediment transport will be higher for stratified conditions, in part due to the change in the sediment profile and in part due to the the change in the velocity profile. Using three methods of extrapolation (constant, linear, and match to zero at the bed) for the velocity profile, the sediment transport estimates for stratified cases range from two to six times those for the unstratified case.

For cross-shelf transport driven by cross-shelf winds, it is not clear if a stratified or unstratified velocity profile, in combination with the respective sediment concentration profile, will drive more sediment transport. Velocity profiles from the 7, 12, and 17-m sites in Figure 4-12 have higher surface boundary layer transports for stratified than mixed conditions, but the stratified profiles also have a return flow centered higher in the water column. The stratified profiles show velocity curving towards zero farther from the bed than the unstratified profiles. Using the three methods of extrapolation, estimates of sediment transport for stratified conditions range from -0.2 to 1.5 times those for the unstratified case. Near bed velocity measurements or 
a wave-current boundary layer model including stratification would be required to make a well informed estimate of sediment transport.

The model in Chapter 5 and previous ones (Austin and Lentz, 2002; Allen et al., 1995; Wijesekera et al., 2003) all show clear asymmetry in the inner shelf circulation and stratification that develop in response to upwelling vs downwelling winds. From the model we can infer that in stratified or summertime conditions, we should expect a preference for onshore sediment transport because of the tendency for upwelling winds to create the stratification that will cap sediment resuspension height and create stronger cross-shelf circulation.

\subsubsection{Mud transport near MVCO}

This chapter was motivated by anecdotal observations of periodic mud deposits near the $12 \mathrm{~m}$ deep MVCO node during previous field experiments in the region by Traykovski et al. (2007). Traykovski et al. noticed scour pits in sandy regions were likely to fill with mud that settled there after wintertime storms. We ask now if the observations from Chapter 4 and model results from Chapter 5 suggest any mechanism that might be responsible for bringing mud to the inner shelf in winter.

In winter, the wind stress at MVCO is typically towards the east southeast (Figure 2-4) and since the water is usually unstratified in winter, it is the cross-shelf component of that wind stress that will drive the cross-shelf circulation. The southward cross-shelf wind stress in winter will drive an onshore transport in the lower portion of the water column. Larger waves in winter time will be able to suspend mud from the bed at deeper sites than smaller summertime waves. Even though the water column is unstratified and some sediment mixes to the surface, the majority will still be in the lower portion of the water column, available for onshore transport. Alongshore nonuniformities in cross-shelf transport are another possible mechanism for bringing mud to MVCO in wintertime. The mud would be made available from the midshelf mud patch by the same larger waves that would make it available for wind-driven circulation, but brought onshore at just one location along the Martha's Vineyard 
inner shelf. The tidally rectified gyre described by Ganju et al. (2011) is an example of one possibility. Mud transported onshore in the eastern side of the gyre could move westward with the predominant along-shore flow before being deposited near MVCO and the SWWIM array.

The model in Chapter 5 and previous ones (Austin and Lentz, 2002; Allen et al., 1995; Wijesekera et al., 2003) all show clear asymmetry in the inner shelf circulation and stratification that develop in response to upwelling vs downwelling winds. From the model we can infer that in stratified or summertime conditions, we should expect a preference for onshore sediment transport because of the tendency for upwelling winds to create the stratification that will cap sediment resuspension height and create stronger cross-shelf circulation. This conclusion seems contrary to the observations of mud moving on to the inner shelf in the winter. However, the increases in transport discussed in Chapters 4 and 5 are increases in transport fraction or transport efficiency. That is, transport relative to the expected neutral density, deep or shallow water transport scales of $u_{*} h$ or $\tau / \rho f$. To first order, inner shelf transport scales with the shear velocity. Average wind stress magnitudes are much larger in winter than in summer and the larger magnitude transports, combined with greater suspension from higher wintertime wave energy are the like causes of higher mud transport across the inner shelf in winter.

\subsection{Summary}

This project took an opportunistic look at sediment resuspension heights through use of an extensive data set of ADCP backscatter intensity collected, along with velocity and density data, as part of the SWWIM field program on the inner shelf south of Martha's Vineyard, MA. The physics discussed is not novel, but its application and importance on the inner shelf are. Despite shallow depths and waves that reach a considerable fraction of water depth, thermal stratification limits the height of sediment resuspension on the inner shelf in summertime. On the Martha's Vineyard 
inner shelf, thermal stratification acts to cap the height of suspended sediment that is most likely composed of flocculated mud. The acoustic and limited optical backscatter compare well with each other and with predictions for sediment concentration profiles made using eddy viscosity estimates from a Mellor-Yamada 2.0 turbulence closure scheme and an assumption of a steady balance between particle settling velocity and upward turbulent diffusion.

Stratification alters the response of both circulation and sediment resuspension to particular wave and wind forcing. By increasing shear in the velocity profile (Fewings et al., 2008) and simultaneously limiting the height of sediment resuspension, stratification can change the magnitude of sediment transport. The stratification could decrease net transport by keeping the sediment lower in the water column, where the velocities are lower on average, or the stratification could increase net transport by confining the sediment to the part of the water column where all the flow is in the same direction. This effect is most likely to be significant in the late summer and fall when strong stratification and large storms are concurrent. These results indicate that thermal stratification should be included in predicting and modeling sediment transport on the inner shelf. 


\section{A Appendix: Regressions between ADCP beams}

Each row shows data from multiple deployments of a single ADCP. SWWIM 2, 4, and 6 all used the same ADCP at the 7-m site. SWWIM 2, 3, 5, 6, and 7 used the same ADCP at the 17 and $27 \mathrm{~m}$ sites. Beams 1 and 2 are show for each site, but all pairs of beams produce similar magnitudes for correlation coefficients and regression slopes and intercepts. Individual beam data is not available for the 12-m ADCP (MVCO node).

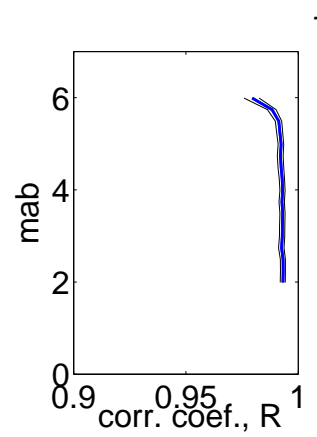

$7 m$ ADCP, beams $1 \& 2$

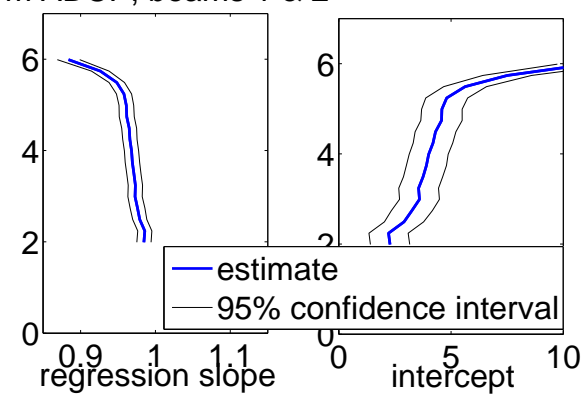

$17 \mathrm{~m}$ ADCP, beams $1 \& 2$
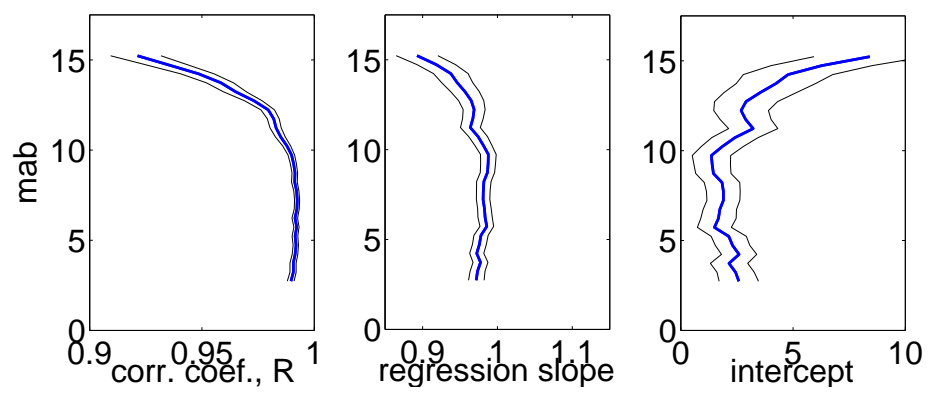

$27 \mathrm{~m}$ ADCP, beams $1 \& 2$
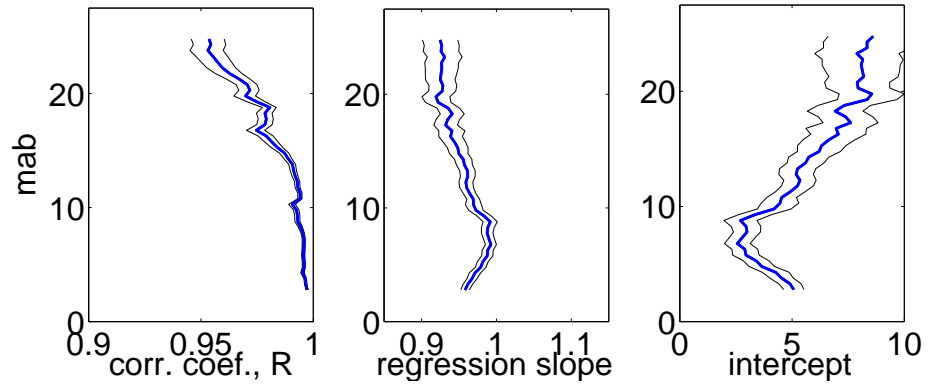

Figure 3-11: Correlation coefficient (left), regression slope (center) and intercept (right) between first and second ADCP beam backscatter intensities from the 7 (top row), 17 (middle row) and 27 (bottom row) meter deep sites.. Variable shown in blue, $95 \%$ confidence intervals in black. 


\section{B Appendix: Optical and acoustic backscatter calibration}

\section{B.1 OBS in the field and lab}

The five OBS deployed during SWWIM were calibrated in a bucket using sediments grab-sampled from the bed of the 17-m site. All showed a linear response to sediment concentration (Figure 3-12). Correlation coefficients, R, are all over 0.99 at a $>99 \%$ confidence level.
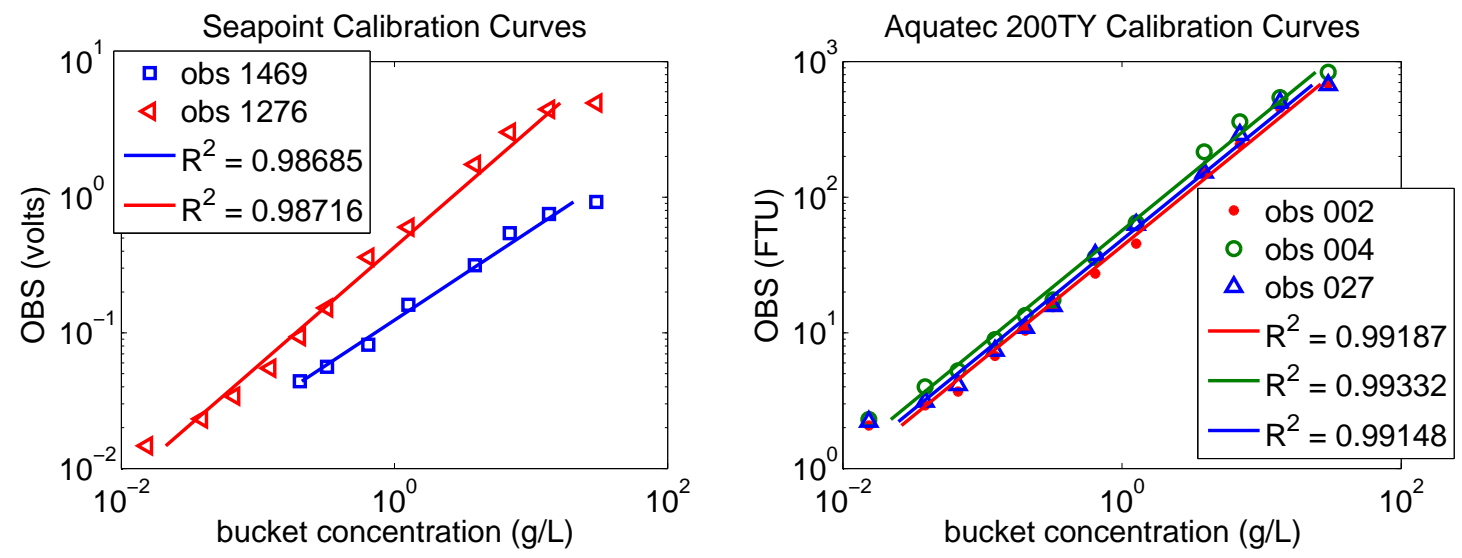

(a) Seapoints mounted on tripods at 17 (red, SN (b) Aquatecs on $17 \mathrm{~m}$ mooring at 5 mab (blue, 1276) and $27 \mathrm{~m}$ site (blue, SN 1469)

SN 027), 7 mab (SN 002), and 13 mab (green, SN 004)

Figure 3-12: OBS calibration curves

In the field, the tripod mounted Seapoints were less consistent. The OBS with a high gain setting often reached the maximum 5 volt response and the one with low gain showed erratic steps and jumps, possibly due to fouling (Figure 3-13(a)). The moored OBS showed excellent correlation with each other and demonstrated the expected relationship between heights above the bed, with the highest sediment concentrations closest to the bed (Figure 3-13(b)). 

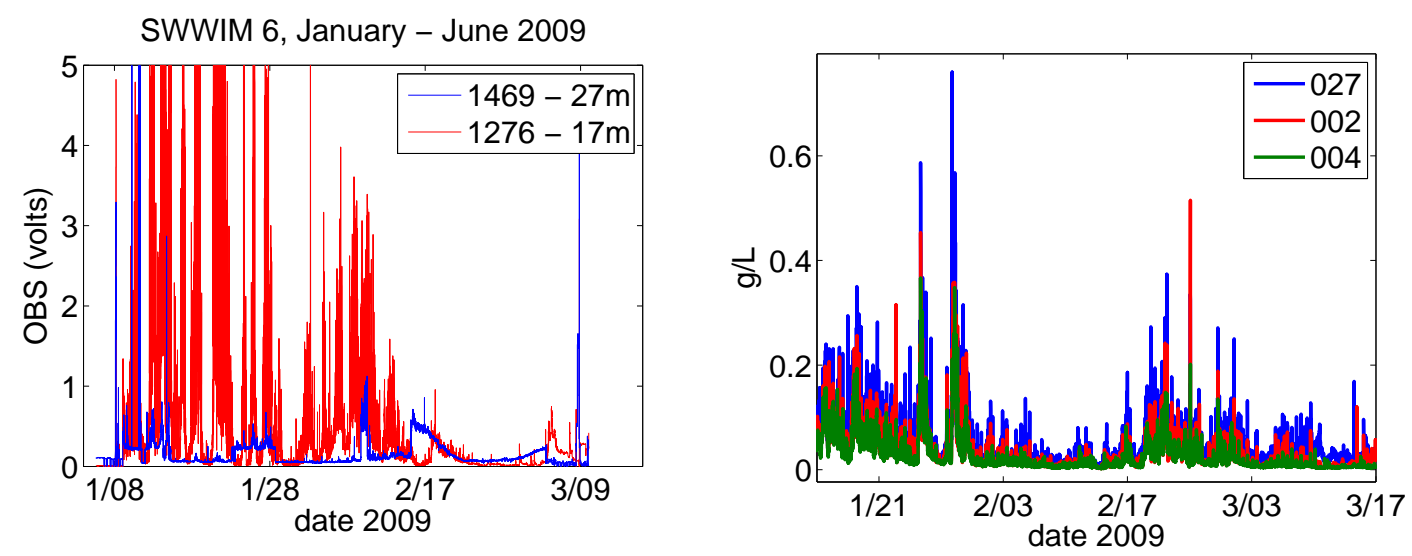

(a) Seapoints mounted on tripods at 17 (red, SN (b) Aquatecs on $17 \mathrm{~m}$ mooring at 5 mab (blue, 1276) and $27 \mathrm{~m}$ site (blue, SN 1469)

SN 027), 7 mab (SN 002), and 13 mab (green, SN 004)

Figure 3-13: Times series of Seapoint voltage (a) and calibrated Aquatec OBS concentration (b)

\section{B.2 Comparing ADCP to OBS}

A regression of the three OBS on the 17-m mooring with their nearest ADCP bins is shown in Figure 3-14. Correlations for individual ADCP bin - OBS pairs, from lowest to highest on the mooring, were $\mathrm{R}=0.54$ (95\% confidence interval of 0.41 $0.66), 0.68$ (0.57 - 0.77), and 0.57 (0.44 - 0.67). The regressions for the same 3 pairs are given by $y=(0.40 \pm 0.11) x+(7.7 \pm 6.2), y=(0.72 \pm 0.14) x+(24 \pm 7.9)$ and $y=(0.40 \pm 0.10) x+(3.9 \pm 5.6)$.

The expectation was that higher in the water column, the regression slope would be higher because suspended sediment there should have a higher fraction of small particles, which the OBS responds to more strongly than the ADCP. Based on the three individual regressions, this effect is not present in this data. The correlation and regression slope of the mid-height ADCP-OBS pair are higher than for the other two pairs, though the correlations are not different at the $95 \%$ confidence level. A single regression with the three pairs combined yields a correlation of $\mathrm{R}=0.52(95 \%$ interval $0.45-0.59)$ with slope and intercept given by $y=(0.46 \pm 0.074) x+(8.7 \pm 4.2)$. The slope of the regression shown in Figure 3-14 is of the same order of magnitude, $\mathrm{O}(1)$, as previously published ones (Kim and Voulgaris, 2003; Wall et al., 2006). Still, 


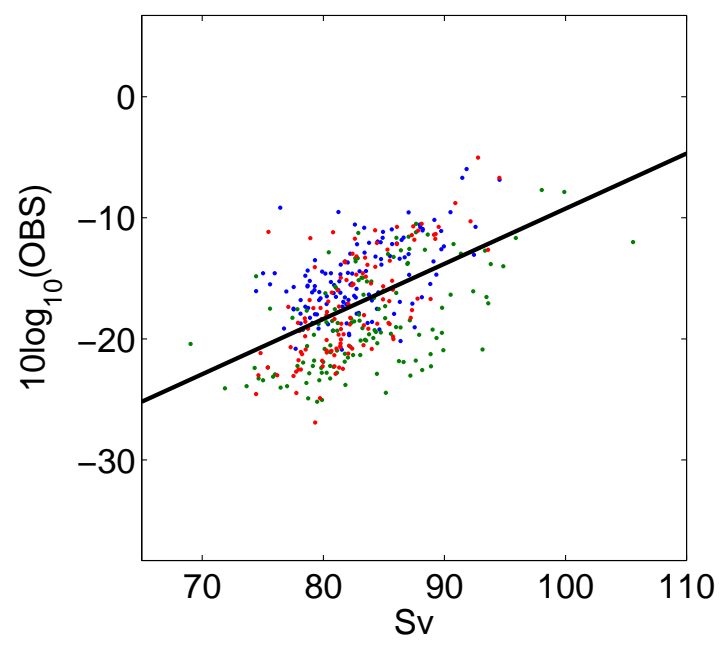

Figure 3-14: Sv and OBS for lower (blue), mid (red) and upper (green) pairs of measurements. Combined regression in black: $\mathrm{R}=0.52$ (95\% interval 0.450 .59 ), $\mathrm{y}$ $=\left(\begin{array}{ll}0.46 & 0.074\end{array}\right) \mathrm{x}+(8.74 .2)$

we are not confident that the bucket calibration accurately represents field sediment concentration so we only use the limited optical measurements to confirm that the acoustic data is qualitatively reasonable. 


\section{Chapter 4}

\section{Observations of the effect of stratification on wind-driven cross-shelf circulation and transport on the inner shelf}

\subsection{Introduction}

Transport across continental shelves allows the exchange of heat, nutrients, larvae, sediment, and pollutants between coastal ecosystems and the open ocean. The inner shelf is a critical link in the cross-shelf transport pathway, connecting the surfzone and the continental shelf, but the mechanisms that drive cross-shelf circulation on the inner shelf are not well understood.

On the mid- and outer shelves, along-shelf winds typically drive cross-shelf transport, following classic Ekman (1905) theory. At shallower depths, where the surface and bottom boundary layers overlap, momentum from the wind mixes to the bottom faster than the Coriolis acceleration can turn it, so the along-shelf wind stress is ineffective at driving cross-shelf Ekman transport. This region, where the entire water column is filled by turbulent surface and bottom boundary layers, is referred to here 
as the inner shelf. On the inner shelf, cross-shelf wind stress has been shown to drive cross-shelf circulation (Fewings et al., 2008) in unstratified conditions. The responses to along-shelf (Austin and Lentz, 2002) and cross-shelf (Tilburg, 2003) wind stresses on an initially stratified inner shelf have been predicted by numerical models but neither prediction has been confirmed with field observations. This study uses three years of observations to describe the inner shelf response to cross- and along-shelf wind stresses in stratified conditions.

\subsubsection{Along-shelf wind stress background}

Ekman (1905) used a constant eddy viscosity to solve for cross-shelf transport by along-shelf wind stress for deep and shallow water and the linear transition between them. In deep water, the solution for cross-shelf transport in the surface boundary layer, $U_{E k}=\tau^{y} /(\rho f)$, is independent of the vertical structure of the profiles of eddy viscosity or velocity. An along-shelf wind stress drives transport towards or away from the coast and the movement of water mass sets up a cross-shelf pressure gradient. A geostrophic along-shelf flow develops to balance the surface pressure gradient and this interior along-shelf flow moving over the bottom causes bottom stress and hence a bottom Ekman layer to develop. In steady state, the transport in the bottom boundary layer is equal but in the opposite direction from the surface Ekman transport, so the no flow through the coast condition is satisfied.

Where the water is shallow enough that the surface and bottom boundary layers overlap, there is a divergence in the Ekman transport within each layer, which leads to upwelling or downwelling. In the shallow water limit, no turning occurs before the surface stress mixes to the bottom so there is no cross-shelf velocity component associated with the along-shelf wind stress; the along-shelf wind stress drives only along-shelf flow. With observations from the same inner shelf on which this study is based, Fewings et al. (2008) found near zero cross-shelf transport driven by alongshelf wind stress, demonstrating the ineffectiveness of along-shelf wind at driving cross-shelf transport on an unstratified inner shelf. For the unstratified case, the 
response to upwelling and downwelling wind stress is symmetric.

On a stratified inner shelf, the response to up- and downwelling wind stresses is strongly asymmetric (Austin and Lentz, 2002). Downwelling fills the inner shelf with constant density water from the surface boundary layer, while upwelling circulation brings up denser water below lighter water, creating stratification. Stratification separates the upper and lower boundary layers, increasing the fraction of full Ekman transport and extending the reach of along-shelf wind as a mechanism for cross-shelf transport into shallower regions (Lentz, 2001; Weisberg et al., 2001; Austin and Lentz, 2002; Kirincich et al., 2005). On the North Carolina inner shelf, Lentz (2001) observed a two-layer response in stratified conditions, with surface and bottom boundary layer transports proportional to wind and bottom stresses, respectively, while the unstratified response had much reduced Ekman transport. Weisberg et al. (2001) compared results of a 3D numerical model of the West Florida Shelf to field measurements and found realistic stratification was required for the model predictions to match the observations. Using an idealized 2D model, Austin and Lentz (2002) described the cross-shelf structure of an upwelling and a downwelling front and found that onshore of the front, the cross-shelf transport was slightly stronger for upwelling than downwelling because of the small stratification the upwelling drew up onto the inner shelf. Several observational attempts have been made to quantify the relationship between the strength of stratification and cross-shelf transport, but there is not yet a consensus. Kirincich et al. (2005) found a weak linear correlation between fraction of Ekman transport and total stratification, while Kirincich and Barth (2009) found it was the eddy viscosity and not the strength of the stratification that controlled the wind's transport efficiency. Recently, Dzwonkowski et al.'s (2011) observations from the Alabama shelf showed a positive relationship between strength of stratification and transport efficiency for low stratifications, but no clear trend for higher stratifications. 


\subsubsection{Cross-shelf wind stress background}

Far less attention has been paid to cross-shelf wind stress as a mechanism for driving cross-shelf transport than to along-shelf wind stress. Only recent studies have focused on cross-shelf wind stress as a mechanism for cross-shelf transport even though work focused on along-shelf wind stress (e.g. Austin and Lentz, 2002) has shown the limitation of upwelling and downwelling to drive cross-shelf transport all the way to the surfzone, and the theoretical basis for the dynamics was laid over a hundred years ago (Ekman, 1905). Regional scale studies have suggested cross-shelf winds as potentially able to complete the transport pathway from surfzone to midshelf. An unstratified model of offshore winds on the West Florida Shelf (Li and Weisberg, 1999a,b) generated a pressure gradient/cross-shelf wind stress balance on inner shelf and Cudaback et al. (2005) showed cross-shelf circulation was driven by cross-shelf wind stresses offshore of a mountain gap near Point Conception, CA. Using an unstratified implementation of a 2D analytic model, Estrade et al. (2008) found that in combination with and along-shelf wind stress, the cross-shelf component of wind drives a nearshore pressure gradient that affects the upwelling circulation.

Recent observational (Fewings et al., 2008) and modeling (Tilburg, 2003) studies focused specifically on the cross-shelf wind stress and have shown it to be a significant mechanism for cross-shelf transport on the inner shelf. Shortly following Austin and Lentz's 2D, idealized, numerical model of cross-shelf circulation driven by alongshelf wind stress, Tilburg (2003) used a similar 2D idealized numerical model to demonstrate cross-shelf wind stress as a viable mechanism to drive transport across the inner shelf. When wind stress blows towards the coast, water near the surface moves onshore. As water piles up at the coast, a pressure gradient grows to balance the wind stress, and that pressure gradient drives an offshore return flow in the lower portion of the water column, a result consistent with Ekman's 1905 solution. For offshore winds, the circulation is reversed, with offshore flow near the surface

and an onshore return flow below. Fewings et al. (2008) described observations of this circulation using wintertime measurements from the Martha's Vineyard Coastal 
Observatory (MVCO). In unstratified conditions at $12 \mathrm{~m}$ water depth, Fewings et al. (2008) found the cross-shelf wind stress to be far more effective at driving crossshelf transport than along-shelf wind stress. Using a single vertical profile in a 2D numerical model, Fewings et al. (2008) also predicted the region where cross-shelf wind is dominant should extend to around $30 \mathrm{~m}$ water depth for a $0.1 \mathrm{~Pa}$ wind stress in unstratified conditions.

Though continental shelves are typically stratified for much of the year, the inner shelf response to cross-shelf winds in stratified conditions is still unclear. In water deeper than the boundary layer thickness, Tilburg (2003) found stratification decreased cross-shelf transport by cross-shelf winds by limiting the depth of the surface boundary layer, causing the Ekman spiral to turn back on itself sooner. However, in that model, momentum did not penetrate into the stratified region of the shelf. Density and momentum were mixed to the same depth, and all circulation was confined to the surface mixed layer, so a stratified inner shelf never developed. Tilburg's study did not address how a stratified inner shelf could develop or what might happen on one.

South of Martha's Vineyard, MA, Fewings et al. (2008) noticed an increased vertical shear in the cross-shelf circulation and, for offshore wind stress, an increased transport in summertime. Summer is when their field site is typically stratified, but Fewings et al. (2008) did not have density data to accompany the velocity measurements. One other recent observational study (Dzwonkowski et al., 2011) used seasonal averages of velocity and density from the Alabama shelf to conclude that cross-shelf wind were most clearly correlated with cross-shelf flow in the fall and winter, when the water was least stratified.

\subsubsection{This chapter}

Here, we use observational methods to describe the effect of stratification on crossshelf circulation and transport by cross- and along-shelf winds on the inner shelf. Previously, only limited observations have captured the variation in transport magni- 
tude and the vertical structure of cross-shelf circulation in response to stratification or cross-shelf wind direction. This study focuses on the local response of a stratified water column and how that response varies as a function of wind direction, stratification, and water depth.

In this chapter, section 4.2 describes the data set and numerical methods; sections 4.3 and 4.4 present the responses to along- and cross-shelf wind stresses; section 4.5 discusses dynamics driving the inner shelf circulation; section 4.6 concludes with a summary of the results.

\subsection{Data and Methods}

\subsubsection{SWWIM}

The data for this analysis come from the SWWIM experiment and the Martha's Vineyard Coastal Observatory (MVCO), which are described in Chapter 2 of this thesis. The MVCO Node and three additional tripod-mounted pressure sensors and upward looking ADCPs were collocated with four temperature and conductivity moorings in $7,12,17.5$, and $27.5 \mathrm{~m}$ water depth at $0.4,1.5,3.8$, and $11.1 \mathrm{~km}$ from shore. These tripods and moorings provide a three year time series of velocity and density profiles across the inner shelf. The Node and ASIT instruments supply wave and wind date for the same time period. All data are interpolated onto the same 20-minute time base.

\subsubsection{Data processing}

The goal of the data processing is to isolate the effects of cross- and along-shelf wind stresses on cross-shelf velocities. The steps are outlined here and described in greater detail in the subsections below. Eulerian measurements do not capture the onshore wave transport above the wave troughs, but do capture the undertow below the moving surface. To avoid biasing the depth-averaged velocity toward the 
offshore direction, we subtract estimates of wave undertow from the velocity profiles. After subtracting wave undertow, we convert the vertical coordinate of the velocity data to a surface-relative value to allow easier comparison of measurements from the same distance below the surface. Next, we remove the barotropic tide from the velocity data. A depth-averaged and low pass filtered velocity is used to determine the proper orientation for along-shelf and cross-shelf axes via principal component analysis. The depth-binned velocity and wind stress data are rotated into the new coordinate system. Unless otherwise noted, all variables are low-pass filtered before the final step of each analysis in this study.

\section{Removing the wave-driven flow}

We remove a wave undertow profile of $u_{w}=-u_{S t}$, as described by Lentz et al. (2008), where

$$
u_{S t}=\frac{H_{s i g}^{2} \omega k}{16} \frac{\cosh [2 k(z+h)]}{\sinh ^{2} k h}
$$

is the Stokes (1847) velocity in the direction of wave propagation. Pressure time series from each ADCP location are used to make time series of water depth. Significant wave height, $H_{\text {sig }}$, dominant wave period, and wave direction, are calculated at the MVCO Node and described on the MVCO website. These theoretical undertow profiles are based on unstratified conditions. Lentz et al. (2008) observed the wave undertow profiles to be more sheared in summertime, so we may be underestimating the near-surface and overestimating the near-bottom velocity contribution from waves but a regression against wave forcing of the velocities with wave undertow previously subtracted (see section 4.2.4 and Appendix 4.A) finds regression slopes not significantly different from zero at the $95 \%$ confidence level throughout most of the water column. 


\section{Vertical coordinate}

The water depth varied with tides and with the exact mooring locations on each deployment so we use a surface-relative vertical coordinate since wind and wave driven flows are strongest and most strongly sheared near the surface. We choose not to use a stretched vertical coordinate that would track $z$ as a fraction of water depth to avoid interpolating across noisy raw data, and because when boundary layer thickness is less than the water depth, it is not clear that total water depth is the appropriate vertical scale.

\section{Detiding}

Barotropic tides are large and dominated by the M2 period. Velocities in each vertical bin are detided for tidal constituents with periods less than six months using T_TIDE (Pawlowicz et al., 2002). Of the tidal constituents that were removed, the fortnightly tide was the longest period with a significant amplitude. Internal tides that are not phase-locked to the barotropic tide remain in the data.

\section{Axes orientation}

Velocities are depth-averaged and low-pass filtered with a 24 hour half-amplitude filter before principal component analysis is performed, following Fewings et al. (2008). Finally, we define along-shelf as the major axis of velocity variation at each ADCP site. The velocities used in all further data analysis have been rotated into along- and cross-shelf components where the positive $y$-axis is roughly eastward and the positive $x$-axis is roughly southward. For the $7,12,17$, and 27 -m sites, the $y$ axes are oriented toward 92, 96, 97, and 102 degrees clockwise from geographic north. Wind stresses

are rotated onto the average of the velocity axes direction so positive along-shore wind stress, $\tau^{s y}$, is $97^{\circ}$ clockwise from geographic north. 


\section{Filtering}

Depth-binned velocity, wind, wave, and density data, and variables computed from them, are low-pass filtered with a 24 hour half-amplitude filter. Filtered time series are subsampled on a 24 hour interval before further analysis. A 24 hour filter is used because diurnal tides and winds are small and the decorrelation time scales for all these variables are near one day.

\subsubsection{Stratification}

\section{Stratification from temperature and conductivity}

The extensive SWWIM data set, described in detail in Chapter 2, has temperature and conductivity measurements from MicroCats as well as temperature measurements from TempPros and Tidbits. Temporal and spatial data coverage is much higher for temperature alone than for density calculations, which require coincident temperature and conductivity. Since there is no freshwater source nearby, temperature variation causes the majority of the density variation, especially in the summertime. Also, when temperature and salinity are correlated, with warmer, fresher water near the shore and surface, and colder, saltier water offshore and below, temperature gradients are an even better predictor of total stratification than the fraction of the physical density variation they cause. This relationship allows us to estimate stratification from vertical temperature gradients when temperature data is available but conductivity data is absent. The steps to create a single $\Delta \sigma_{\theta}$ time series for each mooring site are outlined below. This variable was used as the total water column stratification variable for each mooring site throughout this chapter. Mid water column density jumps used to determine mixed layer thickness later in this chapter are based only on density data and do not use temperature-based estimates of stratification due to inconsistent heights of the instruments between deployments.

The conductivity sensors drift over the course of each deployment and we attempt to adjust the density data to remove trends and jumps. The absolute density value 
varies with seasons, but when the water is well mixed, all density values from a single mooring should vary together. For this reason, we estimate the drift in the density data based on the difference between pairs of sensors. Since we are primarily concerned with the surface mixed layer and density gradients relative to the surface value, we use the topmost density value as our reference and compare each lower measurement to that one. The topmost sensor is also most accurate because it accrued the least biological fouling. We choose times when our temperature measurements indicate that the water column is well mixed by having a top sensor to bottom sensor temperature difference of less that $0.05{ }^{\circ} \mathrm{C}$. For these mixed conditions, a cubic polynomial is fit to the time series of density differences between the topmost and each of the other density time series at each mooring for each deployment. The value of cubic is then subtracted from each density time series for all times, mixed and stratified. The cubic fit is used instead of a linear trend to better account for sudden jumps in the conductivity time series due to fouling.

When and where density data are available, density gradients are used as a measure for total stratification and mixed layer thickness. For the total stratification, the first choice is to use the difference between the highest and lowest density measurements. If the top or bottom sensor data is not available, data from the next highest or lowest sensor is used and the density difference is scaled up based on the reduced distance between the differenced sensors before being included in the time series.

If density data are absent, but temperature data are available, an estimate of stratification based on temperature data is used. This estimate is based on a regression between vertical density gradients and temperature gradients when both are available. With fresher water hugging the coast in both seasons, temperature and salinity are more strongly (anti-)correlated in summertime where fresh and warm water coincide. Since warmer and fresher water both decrease density, temperature gradients are a stronger predictor (steeper regression slope) of stratification in summertime. At each mooring site, we make separate summer (April - Sept) and winter (Oct - March) regressions between vertical density and temperature differences. We 
fill gaps in our total stratification time series with projections of vertical density differences based on these regressions against temperature differences. Typical winter and summer temperature variation is shown in the Chapter 2, section 2.2.3. Figure 4-1 shows summer, winter, and combined (not used in data processing) linear regression between $\Delta \sigma_{\theta}$ and $\Delta T$ at each mooring site.
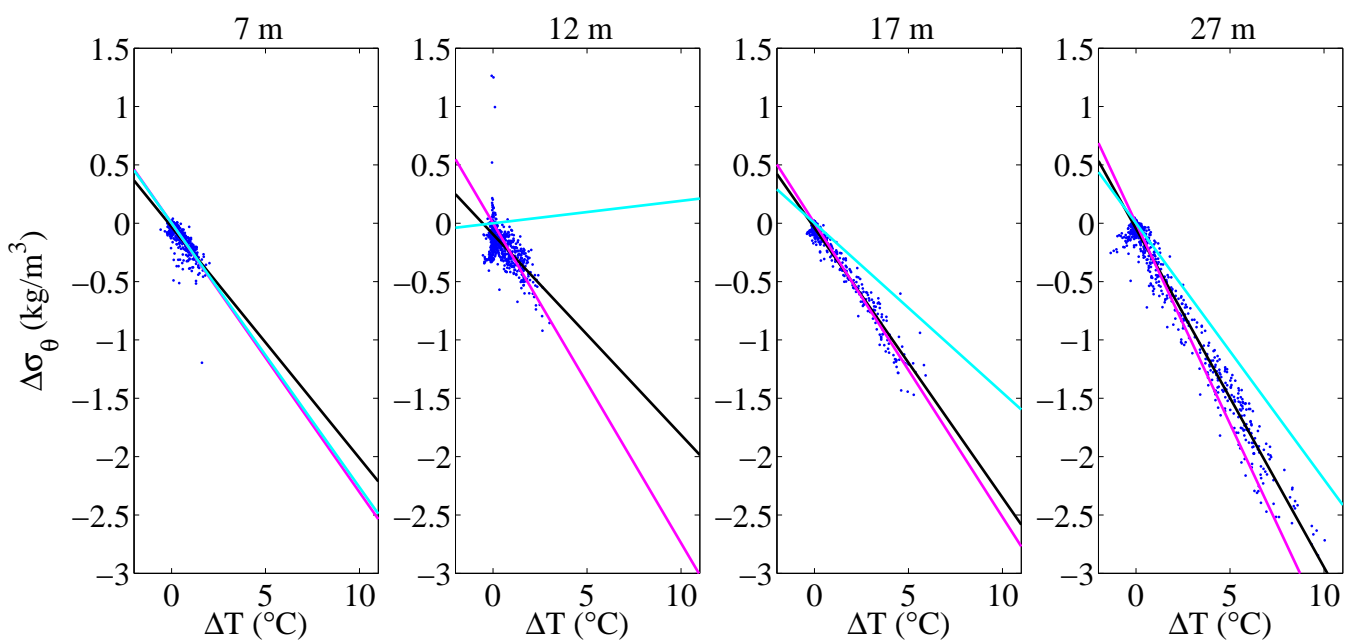

Figure 4-1: $\Delta \sigma_{\theta}$ versus $\Delta T$ and linear regression between then for summer (pink), winter (blue), and combined (black - not used in data processing) at 7, 12, 17, and 27-m mooring sites.

\section{Mixed vs Stratified}

We separate the entire time series from each mooring location into stratified and mixed conditions. Stratified profiles are from times when the density difference between the highest and lowest density measurement is greater than $0.05 \mathrm{~kg} / \mathrm{m}^{3}$, which is near the limit of our measurement sensitivity. Mixed conditions are defined as having a density difference less than $0.05 \mathrm{~kg} / \mathrm{m}^{3}$ between top and bottom measurement heights. We note that internal tides are present and large in the summertime. Particularly at the 7-m, and to some extent at the 12-m site, these internal tides cause the water column to alternate between stratified and mixed on a semi-diurnal tidal frequency. These internal tides are not easily removed because they are not generated locally and so not phase locked to the barotropic tide. When we filter stratification data, we smooth 
over $24 \mathrm{hr}$ periods that contain alternating stratified and mixed time to come up with a representative stratification for the day. We accept that this method may affect the dynamical interpretation of our Eulerian measurements.

\section{Stratification vs mixed layer thickness}

In our density measurements, stratified conditions are present with density gradients continuous to the surface as well as stratified conditions with a mixed layer above a sharp density jump. To test if wind driven circulation is confined to the surface mixed layer, as Tilburg's model predicts, we must limit that analysis to times when our density measurements approximate those generated by Tilburg's model. We choose the surface mixed layer to have less then $0.05 \mathrm{~kg} / \mathrm{m}^{3}$ variation within it and a density jump of $0.05 \mathrm{~kg} / \mathrm{m}^{3}$ or greater between the lowest density measurement within the mixed layer and the next lower measurement (2 to $5 \mathrm{~m}$ below).

Figure 4-2 shows total top to bottom density difference compared to mixed layer thickness at each of the four mooring sites (depicted by color). As described above, density difference shown here is a combination of density data and density difference estimated from temperature alone, when conductivity measurements were not available. The time series of mixed layer thickness is low-pass filtered after the density jump is identified so the resulting time series has a continuous range of layer thickness values rather than discrete ones at density measurement heights. The solid lines are given by $\delta=u_{*} / \sqrt{N f}$, as derived for a slab-model (Pollard et al., 1973) that ignored surface heat flux, advection, or an overlapping bottom boundary layer. Variables used here are average $u_{*}$ for the stratified conditions at each water depth, $\Delta z$ as the height between density measurements at each mooring, $N^{2}=\frac{-g}{\rho} \frac{\Delta \sigma_{\theta}}{\Delta z}, f=10^{-4}$, and SWWIM experiment average $\rho=1024$. Mixed layer thickness monotonically decrease

for larger vertical density difference, following a roughly $1 / \sqrt{N}$ shape as indicated by the theoretical predictions for stratification limiting the depth of wind mixing. The theoretical scales were computed with a constant $u_{*}$ so it is not surprising that the data appear to have a steeper slope, since $u_{*}$ values tend to be higher during times 
of lower stratification and lower during times of higher stratification.

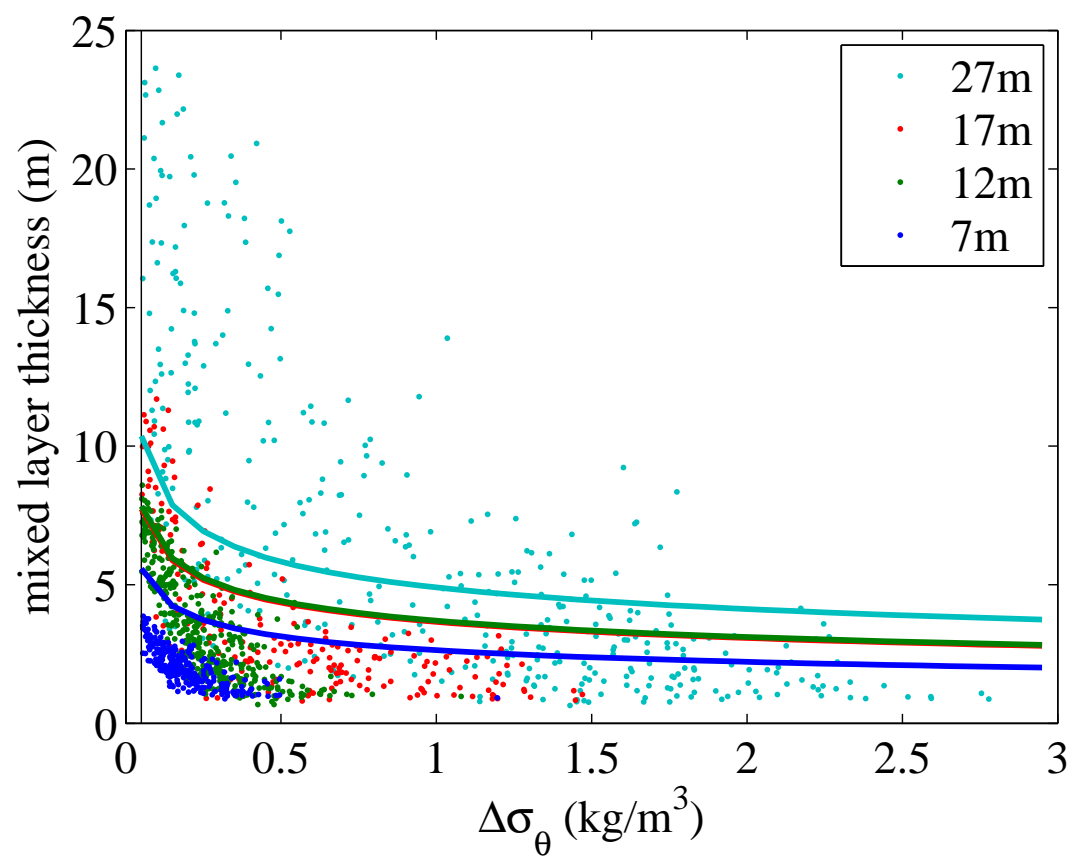

Figure 4-2: Mixed layer thickness vs total $\Delta \sigma_{\theta}$ for stratified conditions with a density jump below a surface mixed layer at $7(\bullet), 12(\bullet), 17(\bullet)$, and 27-m (•) sites. Solid lines of the same color indicate theoretical boundary layer thickness $\delta=u_{*} / \sqrt{N f}$. Vertical black line indicates the $0.05 \mathrm{~kg} / \mathrm{m}^{3}$ cutoff that is used to define stratified conditions throughout this chapter.

Because the strength of the stratification in the interior limits the depth a given wind stress can mix down to, the remaining vertical density difference below the mixed layer is inversely proportional to the thickness of the mixed layer. The density gradient below the mixed layer is shown as a function of mixed layer thickness in Figure 4-3. Profiles with zero $\Delta \sigma_{\theta} / \Delta z$ below the mixed layer have both surface an bottom mixed layers separated by a single density jump, spread no wider that the vertical spacing between our density measurements. 


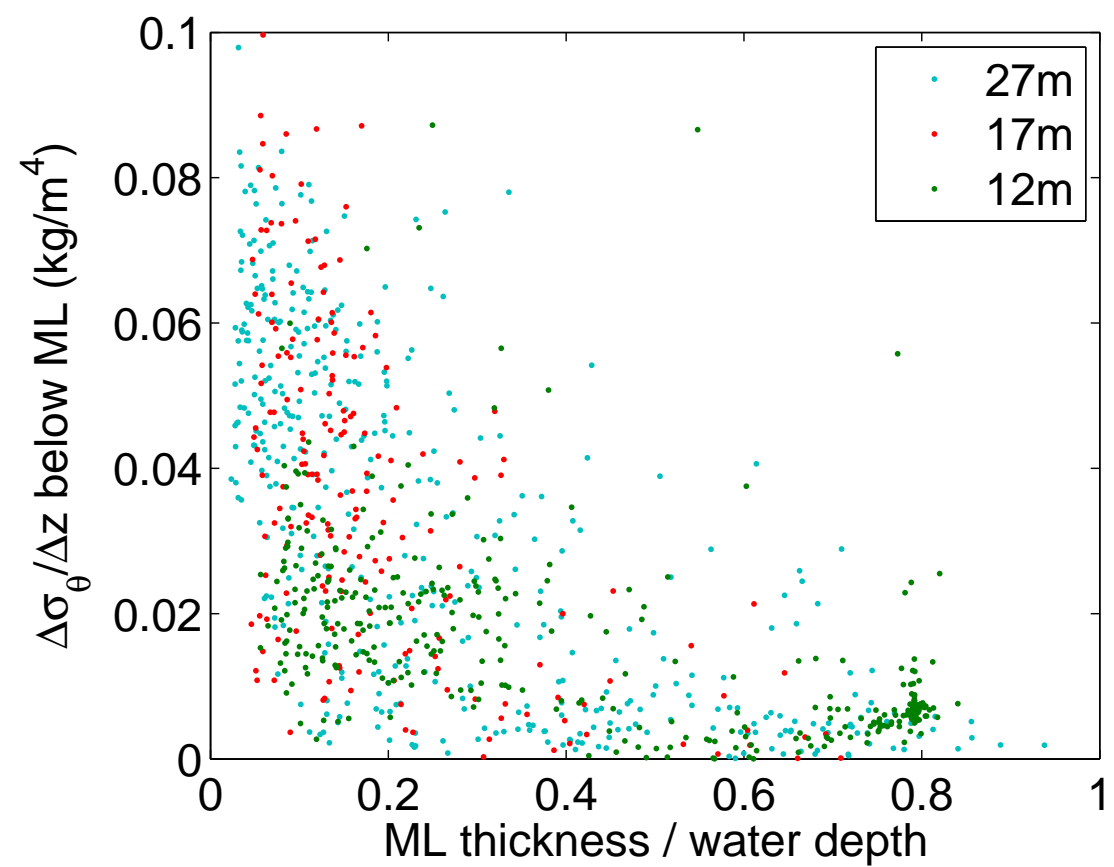

Figure 4-3: Mixed layer thickness as a fraction of water depth vs $\frac{\Delta \sigma_{\theta}}{\Delta z}$ below the density jump at the base of the surface mixed layer at $12(\bullet), 17(\bullet)$, and 27-m $(\bullet)$ sites.

\subsubsection{Regressions with forcing terms}

\section{Regressions on velocity}

A multiple linear regression is used to relate cross-shelf velocity, $u$, at each ADCP bin to various forcing terms that each have units of $\mathrm{m} / \mathrm{s}$. The predictor variables are shear velocity from cross-shelf wind stress, $u_{*}^{x}$ and shear velocity from along-shelf wind stress, $u_{*}^{y}$, and a constant. The multiple regression is a least squares fit to

$$
\begin{array}{ll}
u=a u_{*}^{x}+b u_{*}^{y}+d & \\
\text { where } & u_{*}^{x}=\frac{\tau^{s x}}{\left|\tau^{s x}\right|} \sqrt{\frac{\left|\tau^{s x}\right|}{\rho_{\circ} f}} \\
& u_{*}^{y}=\frac{\tau^{s y}}{\left|\tau^{s y}\right|} \sqrt{\frac{\left|\tau^{s y}\right|}{\rho_{\circ} f}}
\end{array}
$$


$\tau^{s x}$ and $\tau^{s y}$ are across- and along-shore components of wind stress. We also performed regressions that included a term proportional to the depth-averaged wave undertow velocity, $\bar{u}_{w}$. In these cases the equation to fit took the form of

$$
\begin{aligned}
& u=a u_{*}^{x}+b u_{*}^{y}+c \bar{u}_{w}+d \\
& \text { with } \\
& \qquad \bar{u}_{w}=-\frac{U_{S t}}{h}=-\frac{g h}{16 c_{p h}}\left(\frac{H_{s i g}}{h}\right)^{2} \cos \theta_{w}
\end{aligned}
$$

$c_{p h}$ is phase speed, $H_{s i g}$ is significant wave height, and $\theta_{w}$ is angle of wave propagation relative to offshore. The regression coefficients, $a, b$, and $c$, are nondimensional velocities. Profiles of these regression slopes represent how cross-shelf circulation responds to wind and wave forcing.

\section{Regressions on transport}

Near a coastal boundary and assuming along-shelf uniform dynamics, there can be no net transport in the cross-shelf direction. However, we are interested in the transport above the first zero crossing of the velocity profile because this overturning structure of circulation will still create a net transport of any tracer or material that is not uniformly distributed throughout the water column. A second type of multiple regression is used to relate wind and wave forcing to cross-shelf transport. ADCP velocity profiles are integrated in $z$ to calculate cross-shelf transport, $U$, which then is regressed against wind and wave transport scales of Ekman transport, $U_{E k}$, and Stokes transport, $U_{S t}$, respectively.

To estimate the transport, first the profiles of velocity (blue line in schematic figure 4-4) are extrapolated to the surface and bottom depths (red dashed line). At the upper end of the profile, we use the top three measurement heights to linearly extrapolate to the surface. At the bottom, we set the velocity to zero and linearly interpolate between the lowest measurement height and the bottom. Depending on the bottom boundary condition assumption, another option would be to linearly extrapolate to the bottom, as we do toward the surface, instead of setting the bottom velocity to 
zero. We have performed the calculations both ways and results do not change with the selection of bottom boundary condition and slight variations between the two sets of calculated transports are well within the $95 \%$ confidence intervals. After extending the profile to the full water depth, we subtract the depth-average (black dashed line) to satisfy the no flow through the coast requirement. Finally, transport (shaded region in Fig. 4-4) is calculated by integrating the profile from the surface to the first zero crossing.

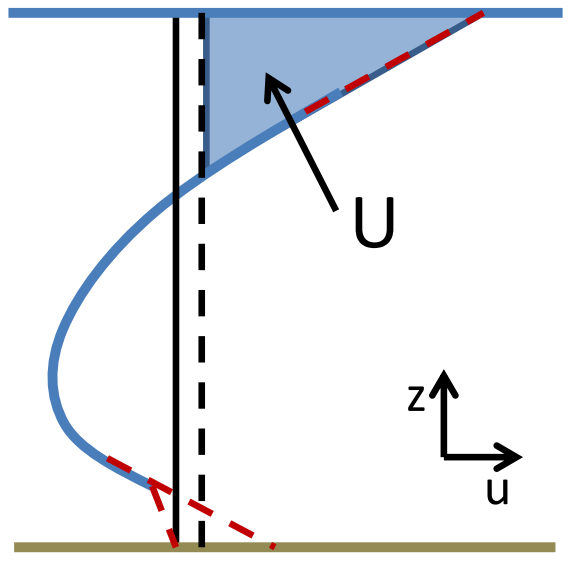

Figure 4-4: Schematic of how velocity profile, $u(z)$, is integrated to get transport, $U$.

The regression, with optional wave term in brackets, takes the form of

$$
U=A U_{E k}^{x}+B U_{E k}^{y}\left[+C U_{w}\right]+D
$$

where

$$
\begin{aligned}
U_{E k}^{x} & =\frac{\tau^{s x}}{\rho_{\circ} f} \\
U_{E k}^{y} & =\frac{\tau^{s y}}{\rho_{\circ} f} \\
U_{w} & =-U_{S t}=-\frac{g H_{s i g}^{2}}{16 c_{p h}} \cos \theta_{w}
\end{aligned}
$$

Regression slopes, $A, B, C$ here are the ratio of two values with units of transport, so represent a transport fraction or nondimensional transport. 


\section{Checking wave removal}

One purpose for subtracting wave undertow profile estimates from the velocity data was to avoid biasing the principal component analysis we used to choose the direction of coordinate axes for wind-driven flow. That removal was imperfect as it was based on inviscid theory and was performed using only the dominant wave period and direction. Since waves and wind are correlated, as a secondary measure to prevent wave undertow velocities from leaking into the wind regression slopes, we keep a wave term in the multiple linear regressions. Regressions between velocity and wind forcing are qualitatively unchanged when performed on velocity data with and without the Stokes velocity removed, and with and without a wave forcing term in the multiple linear regressions. Figure 4-5 shows profiles of regression coefficients from three regressions on velocity from mixed conditions at the 17-m site. Blue lines use velocity that includes wave undertow, fit with equation $4.2 \mathrm{~d}$, which includes a wave-forced term. Green lines use velocity that has had estimates of wave undertow subtracted, fit with equation $4.2 \mathrm{a}$, which does not includes a wave-forced term. Red lines use velocity that has had estimates of wave undertow subtracted, fit with equation $4.2 \mathrm{a}$, which again includes a wave-forced term. The left panel of Figure 4-5 shows the regression slope for the cross-shelf wind term for all three regression methods. The center panel of Figure 4-5 shows the regression slope for the along-shelf wind term for all three regression methods. The right panel of Figure 4-5 shows the regression slope for the wave undertow term for the two regression methods that include it. Profiles of regression coefficients at all four sites for mixed and stratified conditions are available in Appendix 4.A. The red lines represent the regression method used in data analysis throughout the remainder of this chapter. Thin lines are $95 \%$ confidence intervals on the regression slopes.

The wave regression slopes in the right panel show that some undertow velocities did remain in the data after subtracting estimates of those undertows (red lines), but that they are not significantly different from zero throughout most of the water column. Comparison of the red to blue and green lines in the first and second panels 

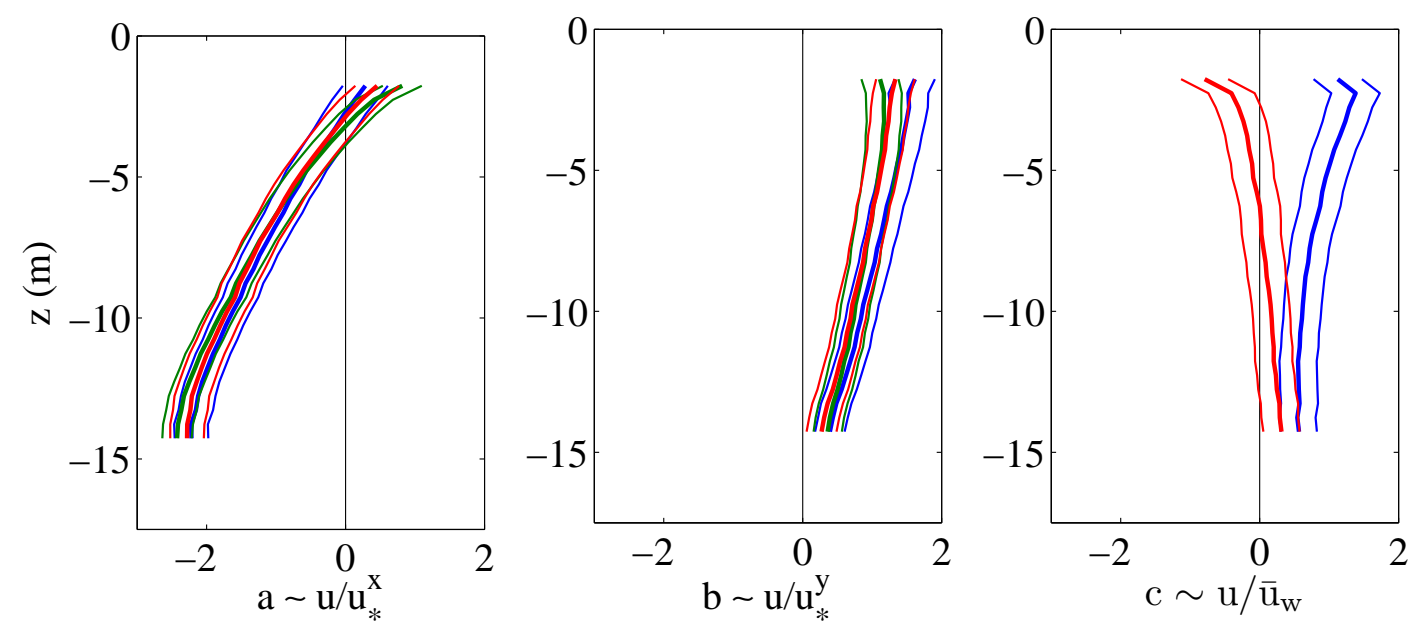

Figure 4-5: Profiles of regression slopes between velocity at each ADCP bin and $u_{*}^{x}$ (left), $u_{*}^{y}$ (center), and $\bar{u}_{w}$ (right) for three regression methods indicated by color: $u$ with undertow, regression includes wave term (blue), $u$ with undertow removed, regression without wave term (green); $u$ with undertow removed, regression includes wave term (red). Thin lines are 95\% confidence intervals on the regression slopes

of Figure 4-5 reassure us that the observed effects of $\tau^{x}$ and $\tau^{y}$ are robust to both the removal of and regression on wave undertow velocities.

\subsubsection{Quasi-1D Model}

A simple numerical model is used estimate a one dimensional (vertical) solution to the steady shallow water equations. The model, described in detail in Lentz (1995) and used similarly in Fewings et al. (2008), assumes no net cross-shelf transport to estimate the cross-shelf pressure gradient. For this study, the model matches surface and bottom boundary conditions given by a prescribed surface wind stress and effective bottom roughness. The eddy viscosity is a cubic profile with a value of zero and linear slopes at both boundaries. The total amplitude is divided by $\sqrt{2}$ to crudely approximate a Mellor-Yamada 2.5 closure scheme for unstratified conditions. 


\subsubsection{Confidence Intervals}

Many of the figures in this chapter depict lines and profiles of regression slopes from multiple linear regressions between velocity or transport and various forcing terms. When a thick line is surrounded by two thinner lines of the same color, the thinner lines represent the $95 \%$ confidence interval on the value of the regression coefficient that is plotted. Unless otherwise indicated, data have been 24-hour low-pass filtered and subsampled before calculating statistics and we assume the individual daily values used in the regression to be independent observations.

\subsection{Along-shelf wind stress}

\subsubsection{Velocity regression profiles}

Profiles of the coefficient of a linear regression between the cross-shelf velocity component, $u$, measured in each ADCP bin and shear velocity calculated from the alongshelf component of wind stress, $u_{*}^{y}$, are representative of the cross-shelf circulation's response to along-shelf wind forcing. The regression coefficient, shown as $b$ in equation $4.2 \mathrm{~d}$, acts as a nondimensionalized velocity, showing the circulation's response to along-shelf wind forcing under various conditions per unit shear velocity. We compare the circulation in mixed to stratified conditions, and for three surface mixed layer thicknesses.

\section{Mixed vs stratified}

When the water is well-mixed (blue lines in Figure 4-6) there is near zero cross-shelf circulation driven by along-shelf wind stresses at all water depths. This observation is consistent with a turbulent boundary layer thickness, $\delta=u_{*} / f$, usually greater than the water depth, which we expect even for weak wind stresses in these shallow, unstratified conditions. For $f=\mathrm{O}\left(10^{-4}\right) \mathrm{s}^{-1}, \rho=\mathrm{O}\left(10^{-3}\right) \mathrm{kg} / \mathrm{m}^{3}$, and water depth $H=\mathrm{O}(10) \mathrm{m}$, we only require a wind stress $\tau=\mathrm{O}\left(10^{-3}\right)$ Pa to reach $\delta>H$. Wind 
stress magnitude is below $0.001 \mathrm{~Pa}$ for only about $1 \%$ of the time at MVCO. These measurements are also consistent with the wintertime observations of Fewings et al. (2008) who found near zero circulation driven by along-shelf winds at MVCO (12-m site) and measurements by Lentz (2001), who found substantially reduced circulation at moorings in 4 to $60 \mathrm{~m}$ water depth on the North Carolina shelf in unstratified compared to stratified conditions.

We notice the profiles from mixed observations are less sheared than those from the 1D model (black lines in Figure 4-6). The difference is likely due to the 1D model's underestimation of mixing, since it only includes wind and not other sources, such as tides. The effect of tides increasing mixing and reducing wind driven cross-shelf circulation in a numerical model is described by Castelao et al. (2010) for surface tides and Kurapov et al. (2010) for internal tides. The 1D model also does not include any effect of suface wave breaking, which is likely to increase mixing when waves, and the winds that generate them, are large.

When the water is stratified (red lines in Figure 4-6), velocity regression profiles from all four mooring sites show a dramatic increase in shear over profiles calculated from times with mixed conditions. This behavior is consistent with the stratified event profiles shown in Lentz (2001). These results also support Fewings et al.'s (2008) statement that more vertically sheared velocity profiles were observed in summertime than wintertime. Comparison to the Austin and Lentz (2002) numerical model will be discussed with the up- vs downwelling comparison in section 4.3.3. Weisberg et al. (2001) found in their model/observation comparison that accurate stratification was required for their numerical model to reproduce the inner shelf circulation. These observation of significant change in the inner shelf circulation driven by along-shelf wind during stratified conditions at Martha's Vineyard broaden the applicability of Weisberg et al.'s result by showing the phenomenon is not particular to the threedimensional bathymetry and circulation of the West Florida Shelf. 

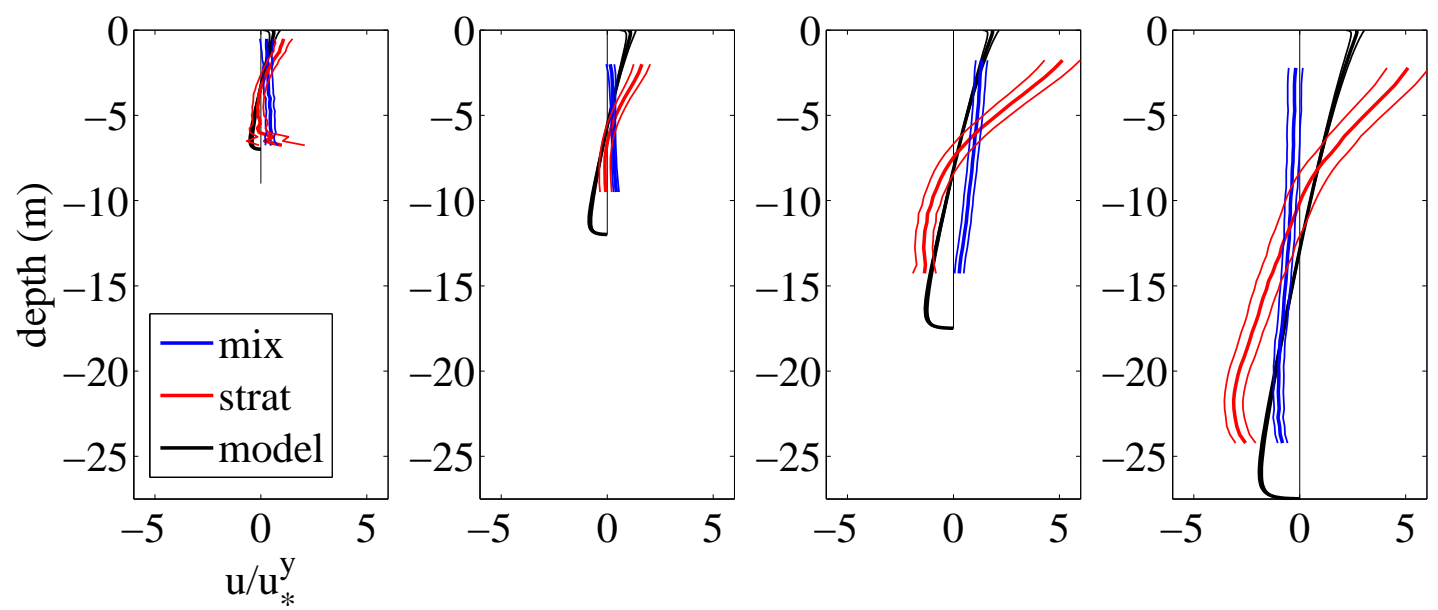

Figure 4-6: Velocity regression slope, $b \sim u / u_{*}^{y}$, for mixed (blue) and stratified (red) conditions, and from a 1D numerical model (black) forced with field measurements of wind stress during mixed conditions.

\section{Mixed layer thickness}

We separate stratified times into groups of various surface mixed layer thicknesses to see if the mixed layer as defined by density also confines the cross-shelf circulation containing the Ekman transport. The relationship between stratification and mixed layer thickness is described in the third part of section 4.2.3 and shown in Figure 4-2. Figure 4-7 shows velocity regression profiles from times with mixed layer thicknesses grouped into $5 \mathrm{~m}$ bins, from 0-5 m (red), 5-10 m (green), and 10-15 m (blue) thick at three mooring sites, water depths of 12,17 , and $27 \mathrm{~m}$. With only three density measurement heights at the 7 -m mooring, we could not observe the variation in mixed layer thickness there.

At all three sites, there are higher first zero crossings and larger surface velocities for thinner mixed layers. For the 0-5 m mixed layer thickness profiles, where the mixed layer thickness is less than half the water depth for all moorings, the shape and zero crossing heights are similar (note that the x-axis scale is smaller for the 12-m site) indicating a similar dynamical balance across all three sites. For all three sites, when mixed layer thicknesses are less than half the water depth, the zero crossing elevation seems to follow the mixed layer thickness. The progression with increasing mixed 
layer thickness was not shown in the similarly directed analysis by Lentz (2001), and only the difference between a two-layer and a continuously stratified scenario was described in Austin and Lentz (2002).

The thinner surface mixed layers and associated higher stratification present a return flow uniformly distributed through the remainder of water depth. Deeper mixed layers with lower total stratification appear to have a more bottom-intensified return flow. This variation in shape in the return flow below Ekman transport agrees with the analysis done by Lentz and Chapman (2004), which indicates that for a fixed bottom slope and Coriolis parameter, a higher stratification should lead to a mid water column return flow throughout a stratified interior while a lower stratification would cause a bottom-intensified return flow in a well-mixed bottom boundary layer.

For all mixed layer thicknesses, the density profiles below the mixed layer are sometimes continuously stratified and sometimes appear to have a bottom mixed layer in addition to the surface mixed layer. The profiles shown in Figure 4-7 include data with continuously stratified interiors, those with surface and bottom mixed layers separated by a stratified interior, and profiles with a single density jump separating surface and bottom mixed layers. Figure 4-3, shows the trend of tending to have stronger stratification remaining below the mixed layer for mixed layers that are a smaller fraction of the water depth. We cannot tell from the value of $\Delta \sigma_{\theta} / \Delta z$ if the interior of the flow has a narrow region of strong stratification above a bottom mixed layer or if the stratification is instead gradual to the bottom. However, interior stratification is equally susceptible to mixing away by bottom friction as by wind stress at the surface so lower vertical density gradients also indicate a higher likelihood of a well-mixed bottom boundary layer.

\subsubsection{Transport fraction}

The coefficient of a linear regression between the cross-shelf transport, $U$, calculated from integrating measured velocity profiles, and Ekman transport, $U_{E k}^{y}$, calculated from the along-shelf component of wind stress, is representative of the cross-shelf 

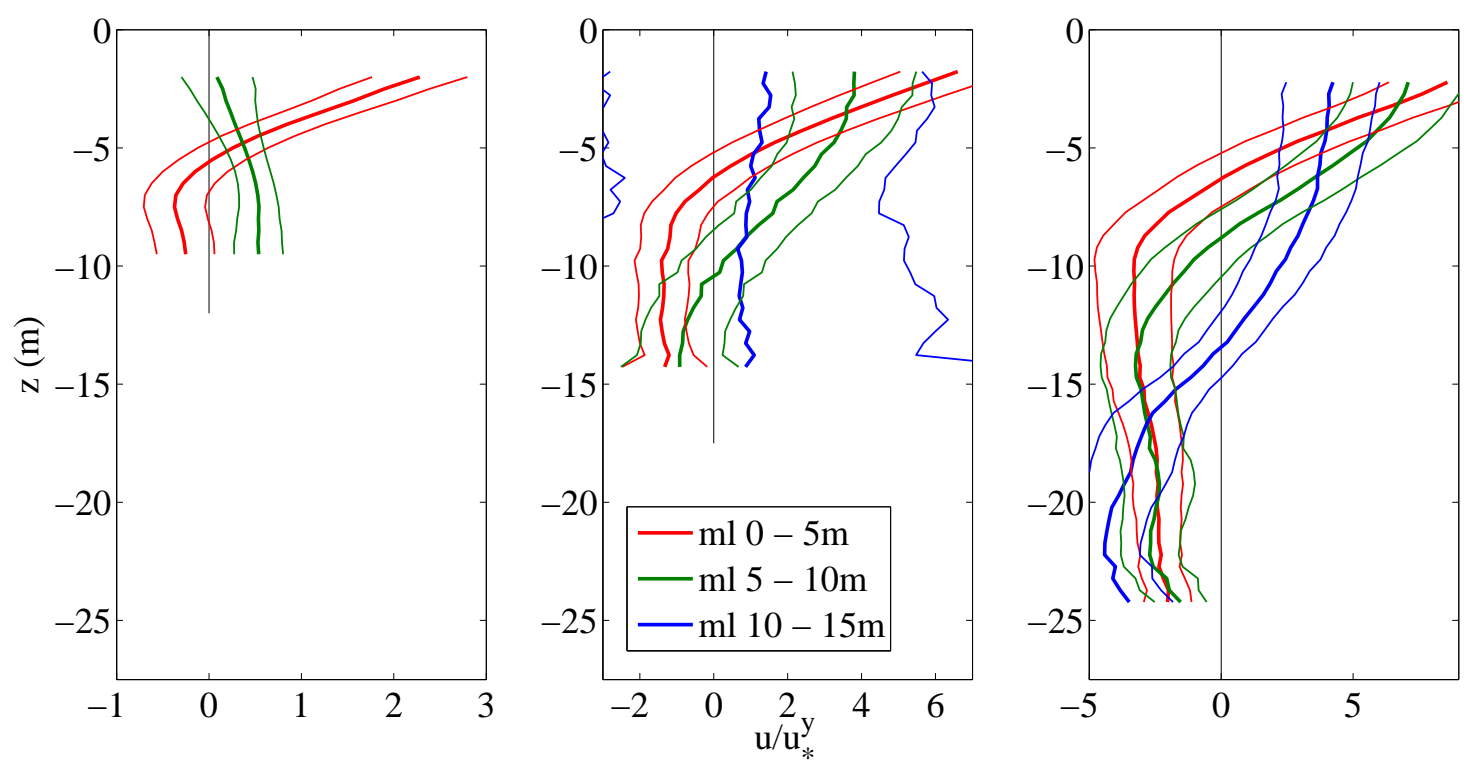

Figure 4-7: Profiles of regression coefficient for $b \sim u / \sqrt{\tau^{s y} / \rho}$ for two mixed layer thicknesses at $12-\mathrm{m}$ site and three mixed layer thicknesses at 17 and $27-\mathrm{m}$ sites. Thin lines are $95 \%$ confidence intervals.

transport's response to along-shelf wind forcing. For each of our four mooring sites, these coefficients, each shown as $B$ in equation $4.3 \mathrm{a}$, represent the fraction of Ekman transport resulting from along-shelf wind forcing under various conditions per unit of wind stress.

\section{Mixed vs stratified}

In Figure 4-8, blue and red lines represent Ekman transport fraction for mixed and stratified conditions, while black and teal lines are both calculated from the unstratified model. The black line is transport calculated using the full height of the model, including velocities very near the surface. Since ADCP measurements stop several meters below the surface, to more closely mimic the data processing, we also calculate transports by truncating the modeled profiles at the height of the top available ADCP bin and linearly extrapolating to the surface as we do with measured velocities. This second method, shown in teal, is an attempt to reduce the overestimation of transport by the model, since we know surface velocities are overestimated due to 
underestimated mixing (ie., no wave breaking or convection at the surface). However, the closeness of the two model lines and their distance from the mixed measurements show that the cause of the discrepancy between regressions of measured and modeled unstratified conditions is not limited to surface sources of mixing, and that the effect of tides over the bottom likely extends throughout the water column.

There is near zero cross-shelf transport driven by along-shelf wind stress during unstratified conditions (blue in Figure 4-8), which is consistent with the results of Fewings et al. (2008). However, we see a dramatic increase in the fraction of Ekman transport under stratified conditions (red lines) where Fewings et al. (2008) noticed only a weak nonzero transport associated with upwelling-favorable winds in the summer. At all water depths, stratification increases cross-shelf transport driven by along-shelf wind stress, but we also see the fraction of Ekman transport increase with water depth from less than $5 \% U_{E k}$ at the 7 -m site up to $30 \% U_{E k}$ at the $27-\mathrm{m}$ site. This increase in transport fraction with water depth indicates that the surface and bottom boundary layers are not completely separated by the presence of any stratification and the surface and bottom boundary layers still overlap out to $27 \mathrm{~m}$ water depth. These observations are consistent with those of Lentz (2001), both in the large increase in transport fraction with the presence of stratification and in the trend of increasing transport fraction with water depth during stratified conditions. The transport fractions for stratified conditions shown here are lower than those reported by Lentz (2001) and Kirincich et al. (2005), but the top-to-bottom density difference cutoff used to define stratified conditions is also lower.

\section{Strength of stratification}

While we used mixed layer thickness as one way to identify the range of stratified conditions in Figure 4-7, we showed earlier, in Figure 4-2, that mixed layer thickness is inversely proportional to total stratification strength. Here, we use total water column stratification, $\Delta \sigma_{\theta} / \Delta z$, calculated between our top and bottom density measurements as described in section 4.2.3, as the independent variable to better 


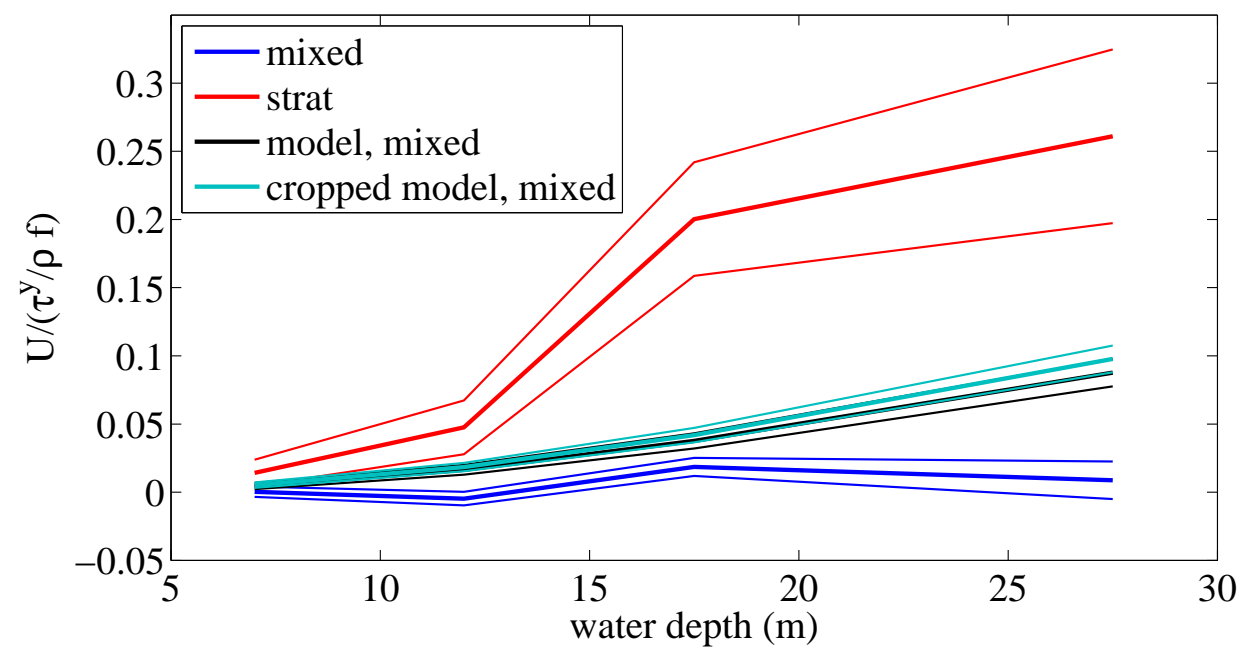

Figure 4-8: $B \sim U /\left(\tau^{s y} / \rho f\right)$ for mixed (blue) and stratified (red) conditions, and from 1D unstratified model, using full depth (black) and cutoff at top ADCP bin height (teal)

compare our observations to previous works. Figure 4-9 shows fraction of Ekman transport by along-shelf wind stress as a function of Brunt-Väisälä frequency squared, $N^{2}=-\left(g / \rho_{0}\right)\left(\Delta \sigma_{\theta} / \Delta z\right)$, calculated in quintiles of stratification strength, excluding mixed conditions, for each of the four mooring sites. Each solid line is the linear fit to the set of five points of the same color.

Two features are apparent in Figure 4-9. First, Ekman transport fraction increases with increasing $\mathrm{N}^{2}$ for a given water depth. Second, transport fraction increases with water depth for a given value of $\mathrm{N}^{2}$. The transport fraction trends towards the theoretical deep water limit of 1 for strong stratification, which is as expected from theory and previous observations by Lentz (2001). This positive correlation between $U / U_{E k}$ and $N^{2}$ supports results from Austin and Lentz (2002) and Kirincich et al. (2005) who found that stratificaion increased transport by along-shelf winds, but is contrary to the finding by Kirincich and Barth (2009) that stratification was not correlated with transport fraction on the Oregon coast.

In deep water or with high stratification, we would expect transport to the right of the wind stress to reach a constant value equal to the full Ekman transport and 


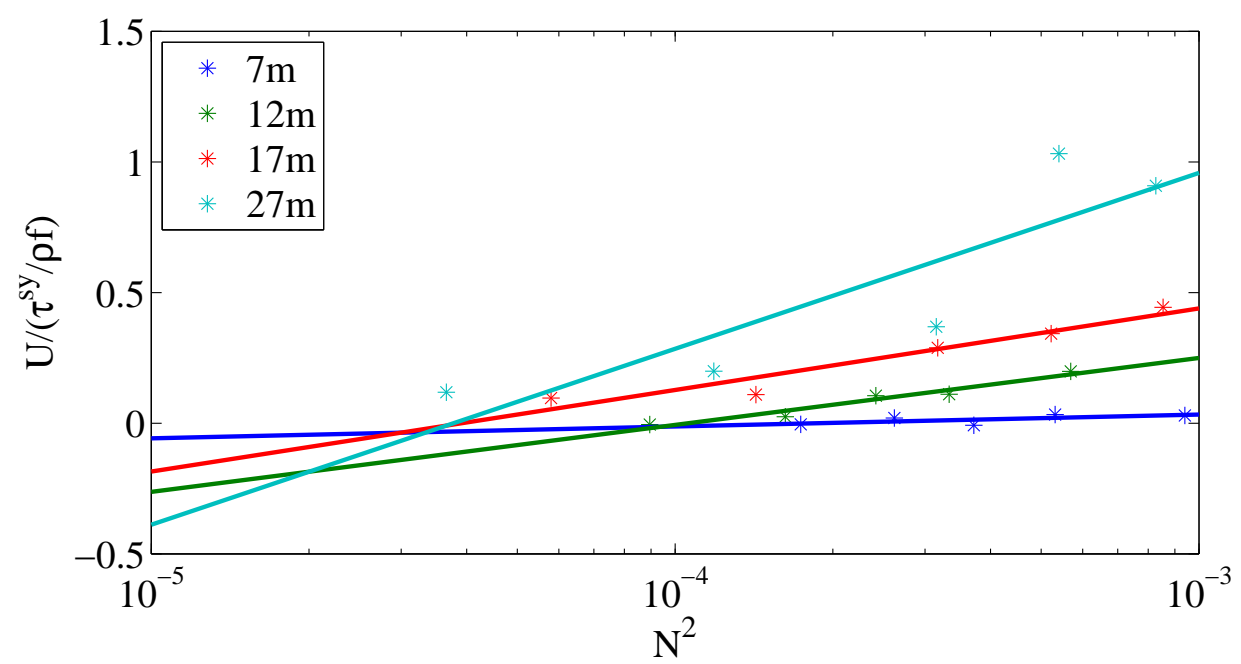

Figure 4-9: $B \sim U /\left(\tau^{s y} / \rho f\right)$ vs $N^{2}$ for 7 (blue), 12 (green), 17 (red), and 27-m (teal) sites. Equations for the linear fits are: $(7 \mathrm{~m}) U / U_{E k}=0.05 \log _{10} N^{2}+0.17 ;(12$ m) $U / U_{E k}=0.26 \log _{10} N^{2}+1.0 ;(17 \mathrm{~m}) U / U_{E k}=0.31 \log _{10} N^{2}+1.4 ;(27 \mathrm{~m}) U / U_{E k}=$ $0.67 \log _{10} N^{2}+3.0$

not change with continued increase in water depth or stratification. Except at the 27-m site, the lack of leveling off of the transport fraction at higher stratifications indicates that the surface and bottom boundary layers are not fully separated, despite the indication that they should be, based on the relationship between total stratification and mixed layer thickness shown in Figure 4-2. These results indicate that all momentum is not contained in surface boundary layer as defined by mixed layer thickness (see velocity regression profiles in section 4.3.1). Other possible sources of error here are that the $24 \mathrm{hr}$ low-pass filtered mixed layer depth may not be good representation of stratification at shallower sites and combining up- and downwelling events may obscure the different magnitudes of the effect of stratification on on- or offshore transport.

\subsubsection{Asymmetry between up- and downwelling wind stress}

For uniform density water, upwelling and downwelling wind stresses theoretically drive equal and opposite circulations, while in stratified conditions, we expect upwelling 
to reduce mixing by bringing up continuously denser water and for downwelling to enhance mixing by forcing lighter fluid under denser. For observations of the effect of stratification, we work on the premise that upwelling and downwelling responses are not symmetric so here we separate times with up- and downwelling forcing to separate the effects of along-shelf wind direction and stratification on cross-shelf circulation and transport.

\section{Velocities}

As observed for the combined up- and downwelling wind stresses in section 4.3.1, there is a near zero cross-shelf circulation in mixed conditions, as show by the blue lines in Figure 4-10, for both up- and downwelling conditions. In the field data, we do not have a perfectly alongshore uniform topography nor perfect removal of wave-driven flows. The realities of field measurements explain some of the difference between our observations and the model of unstratified conditions. The non-zero depth average may be a product of along-shelf variation in the local topography and resulting circulation patterns where net on or offshore wind-driven transport at our measurement site is balanced by an opposing net cross-shelf transport at another along-shore location. The apparent onshore near surface velocity driven by upwelling winds could be caused by incomplete removal of wave driven undertow, or imperfect separation of along- and cross-shelf forcing. This phenomenon is most evident at our 12-m site and was also observed by Fewings et al. (2008), who noticed an onshore transport by upwelling wind stresses calculated from wintertime velocity profiles from the same location at MVCO.

The effect of stratification on the response to upwelling wind stress is clear. At all four sites the surface velocity is significantly higher and the bottom velocity significantly lower for stratified conditions than mixed. We see a similar but smaller change in regression profile shape between mixed and stratified conditions for downwelling wind stress, but the difference is almost entirely confined within the $95 \%$ confidence intervals, which are wider than those for the upwelling cases. The larger effect of 
stratification for up- (vs down-) welling wind stress agrees with observations by Lentz (2001) and Austin and Lentz (2002). Part of the difference in shear between up and downwelling stratified responses may be due to the higher average stratification during upwelling conditions but the wider confidence intervals do not necessarily follow, as the wind stress magnitudes and number of data points are similar. The less-sheared velocities during downwelling conditions indicate an increase in mixing of density as well as momentum, which we expect in downwelling conditions. The wider confidence intervals may also indicate an unsteadiness to downwelling conditions, again in line with our theoretical downwelling scenario.

In the scenario of identical density profiles existing under up- and downwelling wind stresses, we would expect the circulation to respond symmetrically. However, the downwelling model of Austin and Lentz (2002) makes it clear that the density field does not respond symmetrically to up- and downwelling wind stresses. The surprising observation companion to these velocity regressions is the statistics of the frequency of stratified measurements. When the water column is stratified at the 27-m site (we choose this criterion to exclude conditions when there are no strong density gradients in the MVCO region), we would expect the 7, 12, and 17-m sites to also be stratified for a larger fraction of time under upwelling than downwelling wind stresses. However, the measurements do not match this expectation. Under downwelling winds, the 7,12 , and 17 -m sites are stratified 68,80 , and $72 \%$ of the time that the $27-\mathrm{m}$ site is stratified. With upwelling winds and stratification at $27 \mathrm{~m}$, the shallower sites are stratified at rates of 69,74 , and $76 \%$. Apparently, the $12-\mathrm{m}$ site is more likely to be stratified by upwelling than downwelling wind stresses, while the 7 , and $17-\mathrm{m}$ sites are not.

\section{Transport}

Stratification increases the cross-shelf transport for both up- and downwelling wind stresses and both the upwelling and downwelling cases reflect the behavior observed in the combined data set in section 4.3 .2 of increasing separation of mixed and stratified 

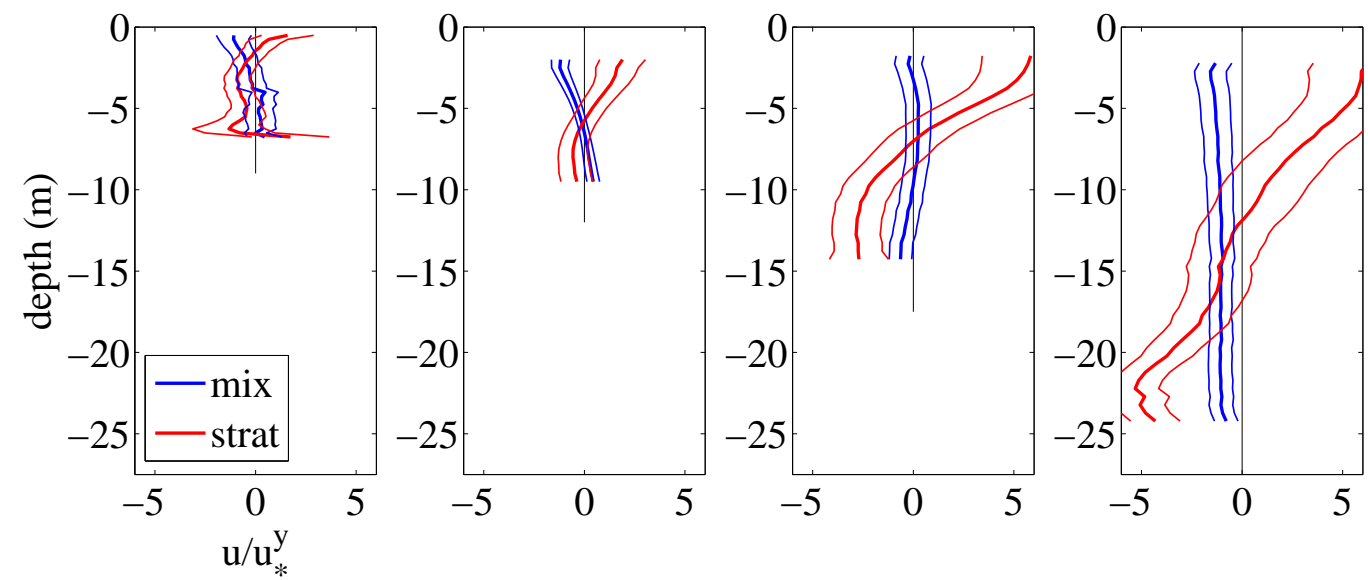

(a) upwelling
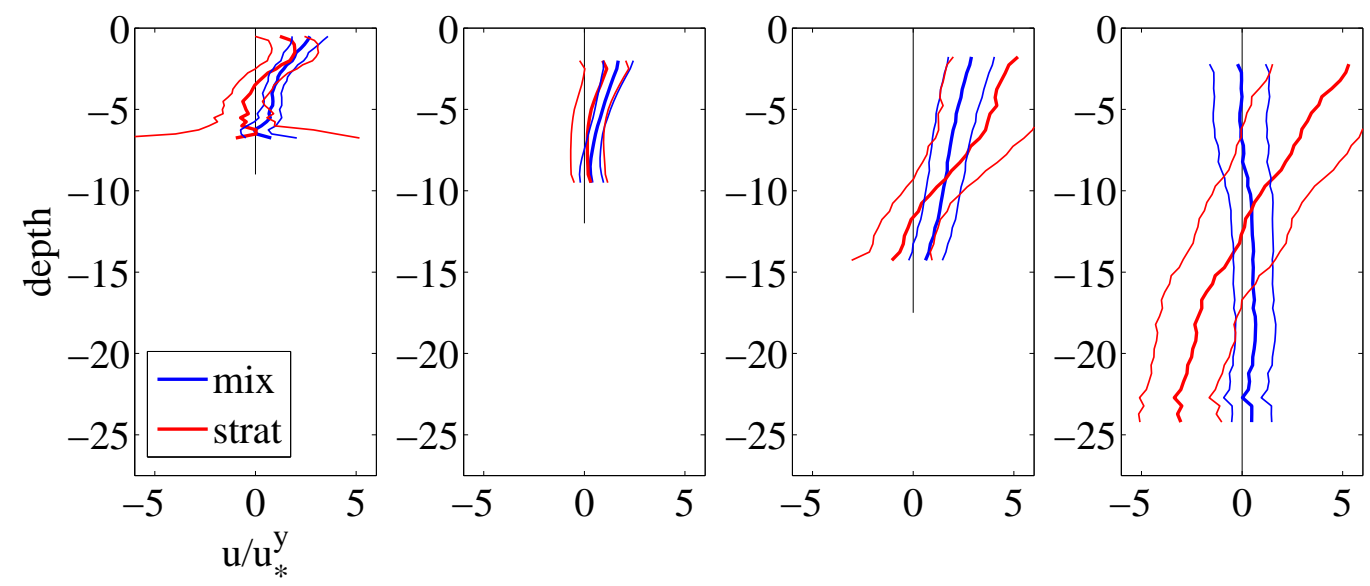

(b) downwelling

Figure 4-10: $b \sim u / \sqrt{\tau^{s y} / \rho}$ mixed (blue) and stratified (red) response to upwelling (top) and downwelling (bottom) wind stress

conditions with water depth. In the upwelling case, the transport is significantly larger for stratified than for mixed conditions at all water depths. In the downwelling case, the trend of increasing transport for stratified conditions is the same, but the separation between mixed and stratified conditions is only significant at the $27-\mathrm{m}$ site.

While the difference between mixed and stratified conditions is larger for upwelling than downwelling conditions, the stratified response is not significantly larger in the upwelling case despite larger average stratification during the upwelling conditions. 
Again, we note that in the mixed, upwelling case we observe the unexpected and probably nonphysical response of onshore (negative) transport from upwelling wind.

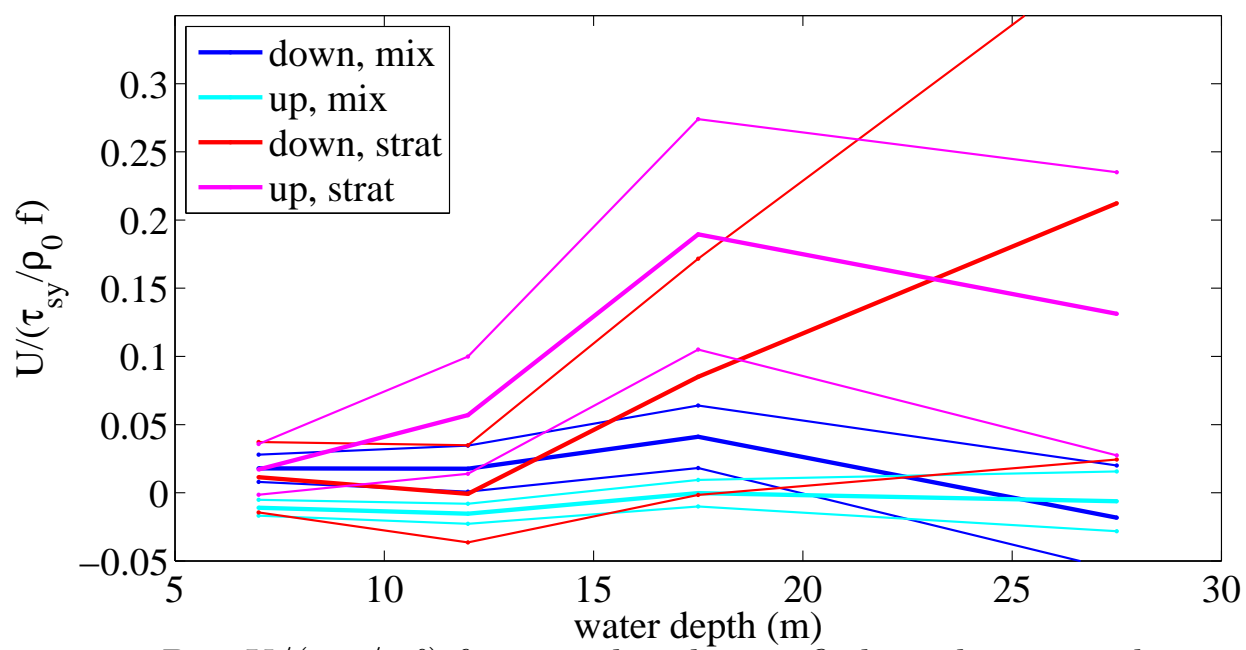

Figure 4-11: $B \sim U /\left(\tau^{s y} / \rho f\right)$ for mixed and stratified conditions with up- and downwelling wind stress

\subsection{Cross-shelf wind stress}

\subsubsection{Velocity regression profiles}

Profiles of the coefficient of a linear regression between the cross-shelf velocity component, $u$, measured in each ADCP bin and shear velocity calculated from the crossshelf component of wind stress, $u_{*}^{x}$, are representative of the cross-shelf circulation's response to cross-shelf wind forcing. The regression coefficient, shown as $a$ in equation $4.2 \mathrm{a}$, acts as a (scaled) velocity, showing the circulation's response to cross-shelf wind forcing under various conditions per unit of wind stress. We compare the circulation in mixed to stratified conditions, and for three surface mixed layer thicknesses.

\section{Mixed vs stratified}

For both mixed (blue) and stratified (red) conditions shown in Figure 4-12, near surface velocities are in the direction of wind stress with return flow over a larger 
portion of the water column below. The profiles from mixed observations are less sheared than those from the 1D model (black). The difference is likely due to the same two factors as described for along-shelf wind stress in section 4.3.1. The 1D model underestimates mixing because it does not include bottom stress from tidal velocities, which are prevalent in the field, especially for the shallower water depths. This effect is most evident throughout the water column at the $7-\mathrm{m}$ site. The 1D model also does not include mixing from wave breaking, which likely increases the eddy viscosity near the surface, especially when waves (and likely also wind-driven circulation) are large.

For the 7,12 , and $17-\mathrm{m}$ sites, the stratified profiles are significantly more sheared than the mixed ones in the upper portion of the water column. The stratified profiles also have higher surface velocities $(12,17 \mathrm{~m})$ or stronger return flow $(7,17 \mathrm{~m})$. The shape of the profiles and the magnitude of the velocities are comparable to those calculated by Fewings et al.'s (2008) binned averaging method. This increase in shear at the shallower sites is also consistent with Fewings et al.'s observation that summer profiles of cross-shelf wind driven circulation were more sheared than winter ones. However, Fewings et al. noticed higher first zero crossing in summer than winter, while we do not see a clear pattern in the direction of change in zero crossing height across our four water depths.

\section{Mixed layer thickness}

For all but the shallowest ( $7 \mathrm{~m}$ deep) mooring site, we are able to separate stratified conditions by the thickness of the surface mixed layer into ranges of $0-5,5-10$, and 10 - 15 meters, shown in Figure 4-13. As shown in section 4.2.3, thinner surface mixed layers are also representative of higher total stratification and regression profiles (not shown) look similar if we instead separate stratified times by total stratification, including times with no measurable surface mixed layer (could be shallower than our top density measurement).

At the $12-\mathrm{m}$ site, there is higher shear in the velocity profile. For either mixed 

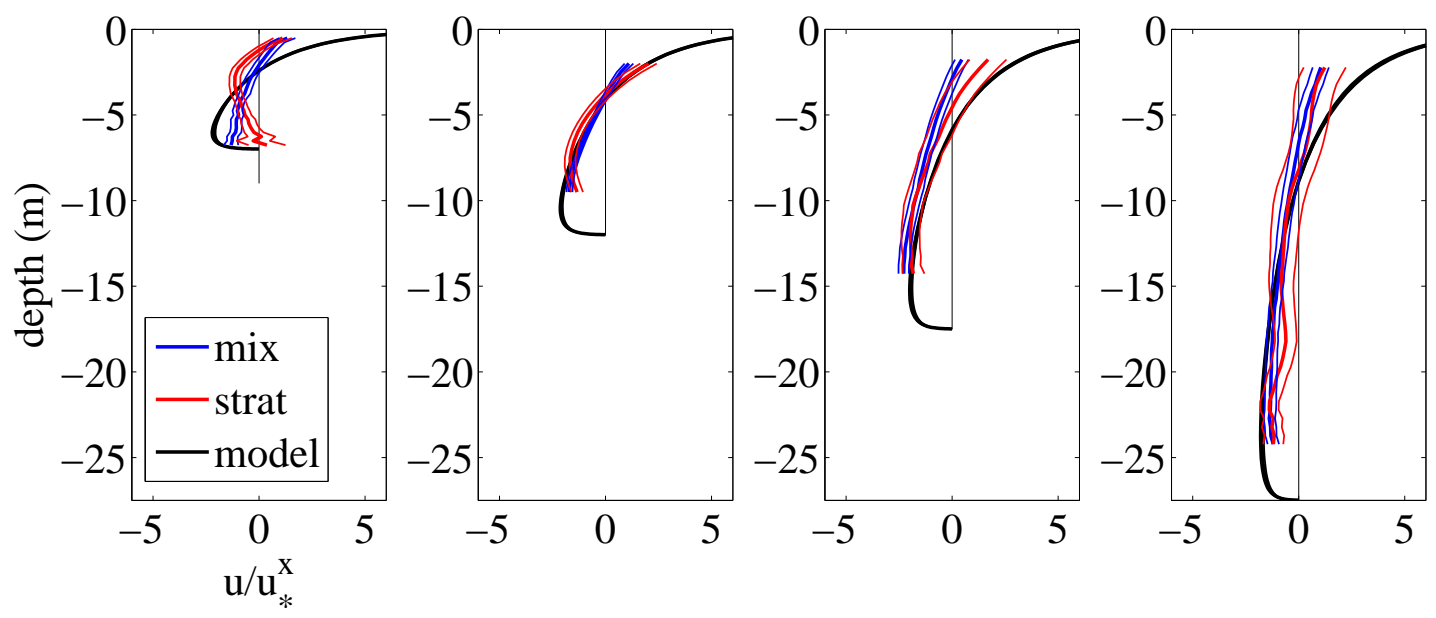

Figure 4-12: Velocity regression slope, $a \sim u / u_{*}^{x}$, for mixed (blue) and stratified (red) conditions, and from a 1D numerical model (black) forced with field measurements of wind stress during mixed conditions.

layer thickness, the regression slopes are non-zero indicating cross-shelf wind stress drives circulation throughout the stratified water column.

At the 17-m site, the confidence intervals are wide for the 10 - $15 \mathrm{~m}$ mixed layer, but this deeper mixed layer circulation does appear to have a higher surface velocity than the thinner mixed layer. The $0-5$ and 5 - $10 \mathrm{~m}$ mixed layers do not have significantly different circulation, but for both these mixed layer thicknesses, there is surface velocity in the direction of the wind and a significantly non-zero return flow throughout the lower portion of the water column.

At the 27-m site, the confidence intervals are wide enough that the velocity profiles for the three mixed layer groupings are not significantly different from each other through most of the water depth. However, the surface velocity is smallest and the first zero crossing highest for the thinnest mixed layer and the surface velocity largest and first zero crossing lowest for the thickest surface mixed layer. The shapes of these profiles are suggestive of an Ekman spiral confined within the surface mixed layer. A thinner surface mixed layer forces the Ekman spiral to turn more tightly, causing this down-wind component of velocity to reverse direction closer to the surface.

The most important feature of the profiles to notice here is that at all depths, especially the shallower ones, there is a non-zero velocity. The stratified response to 
cross-shelf wind stress is not limited to the surface mixed layer as in Tilburg's (2003) numerical model.
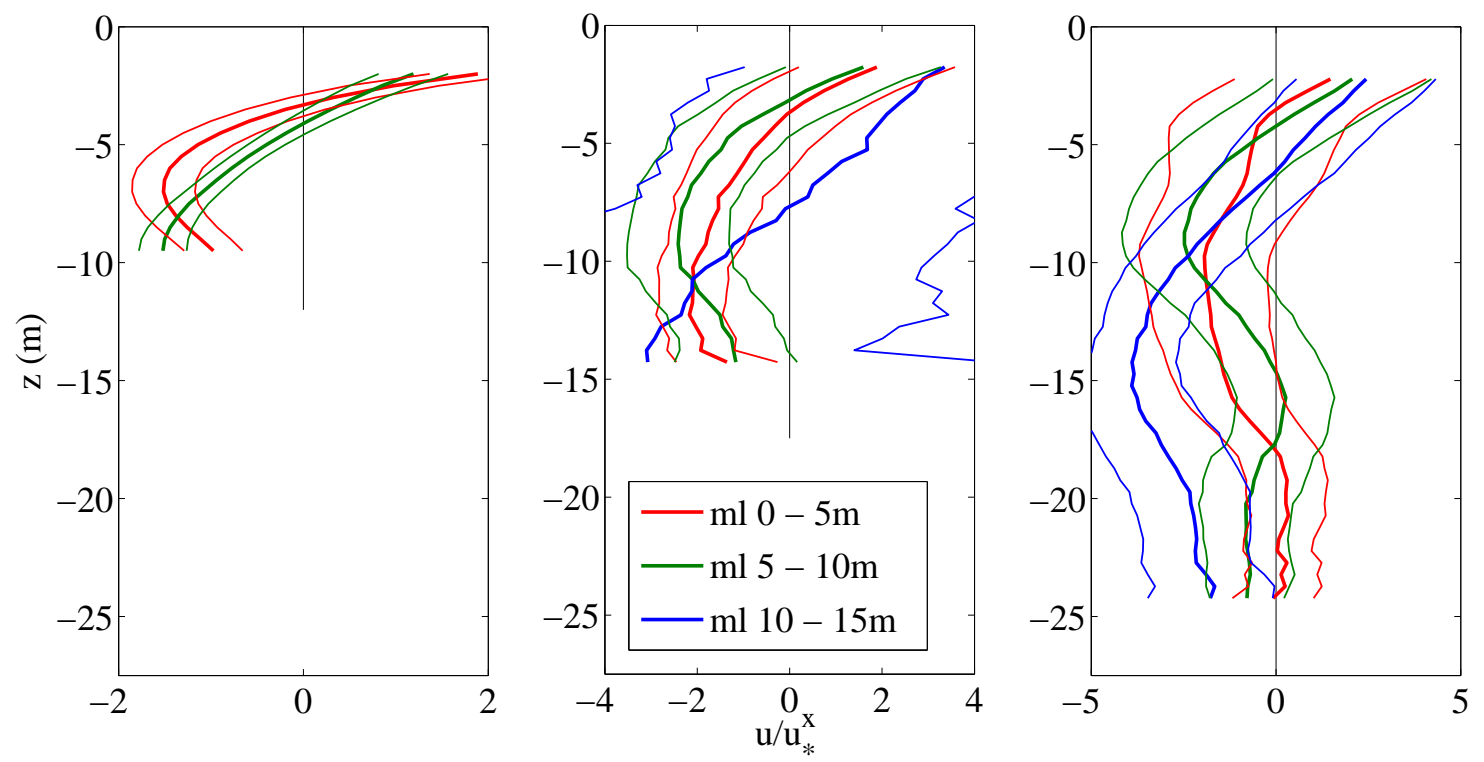

Figure 4-13: Profiles of regression coefficient for $a \sim u / \sqrt{\tau^{s x} / \rho}$ for three mixed layer thicknesses

\subsubsection{Transport fraction}

The coefficient of a linear regression between the cross-shelf transport, $U$, calculated from integrating measured velocity profiles, and Ekman transport, $U_{E k}^{x}$, calculated from the cross-shelf component of wind stress, is representative of the cross-shelf transport's response to cross-shelf wind forcing. A described in section 4.2.4, the calculation is done assuming zero depth-averaged velocity. For each of our four mooring sites, these coefficients, each shown as $A$ in equation $4.3 \mathrm{a}$, represent the fraction of Ekman transport resulting from cross-shelf wind forcing under various conditions per unit of wind stress. 


\section{Mixed vs stratified}

In unstratified conditions, we can expect the cross-shelf transport to increase across the shelf towards the deep water limit as the water depth increases towards the unstratified frictional boundary layer thickness scale of $u_{*} / f$. The deep water limit is $0.32(\tau / \rho f)$ for constant eddy viscosity and $\sim 0.4(\tau / \rho f)$ in the 1D model we use here. In Figure 4-14 the observed transport fraction for mixed conditions (blue) increases with water depth, though stays at about half the value predicted by the 1D numerical model (black). Processing the model output like the data (teal) produces an estimate of transport that is only slightly closer to our observations than from using the full water depth of model output. Since the truncated model data does not produce a much closer match to transport calculations made from the ADCP measurements, we infer that the the higher value predicted using the the model is primarily due to the model underestimating mixing, and not limited range of our ADCP measurements.

In stratified conditions (red in Figure 4-14) we see a significant increase in transport over unstratified conditions for the 12 and $17-\mathrm{m}$ sites. At the 7 and $27-\mathrm{m}$ sites, there is not a significant change at the $95 \%$ confidence level. At the $12-\mathrm{m}$ site, the stratified transport fraction is about twice as large as for mixed conditions, which is similar to the difference between summer and winter observed by Fewings et al. (2008) for offshore wind stress. Here, the on- and offshore wind stresses are combined but will be considered separately in section 4.4.3. The stratified transport fraction increases sharply from 7 to $12 \mathrm{~m}$, and decreases (though not significantly) from the 12 to 17 and 17 to $27-\mathrm{m}$ sites. This leveling off of the stratified transport fraction indicates that the upper and lower boundary layers may be separated, reaching the deep water limit of transport as best we can measure with our height-limited field observations. This observation is surprising, however, when considered in the context of our along-shelf wind stress transport estimates, which suggested that even with high stratification at our deepest site, we had not yet reached fully separated upper and lower boundary layers. However, for along-shelf wind stress, depth of two times the boundary layer thickness is needed for full separation and for cross-shelf wind stress, 
a lower boundary layer does not necessarily develop, so depth of only one boundary layer thickness would be necessary to reach maximum transport.

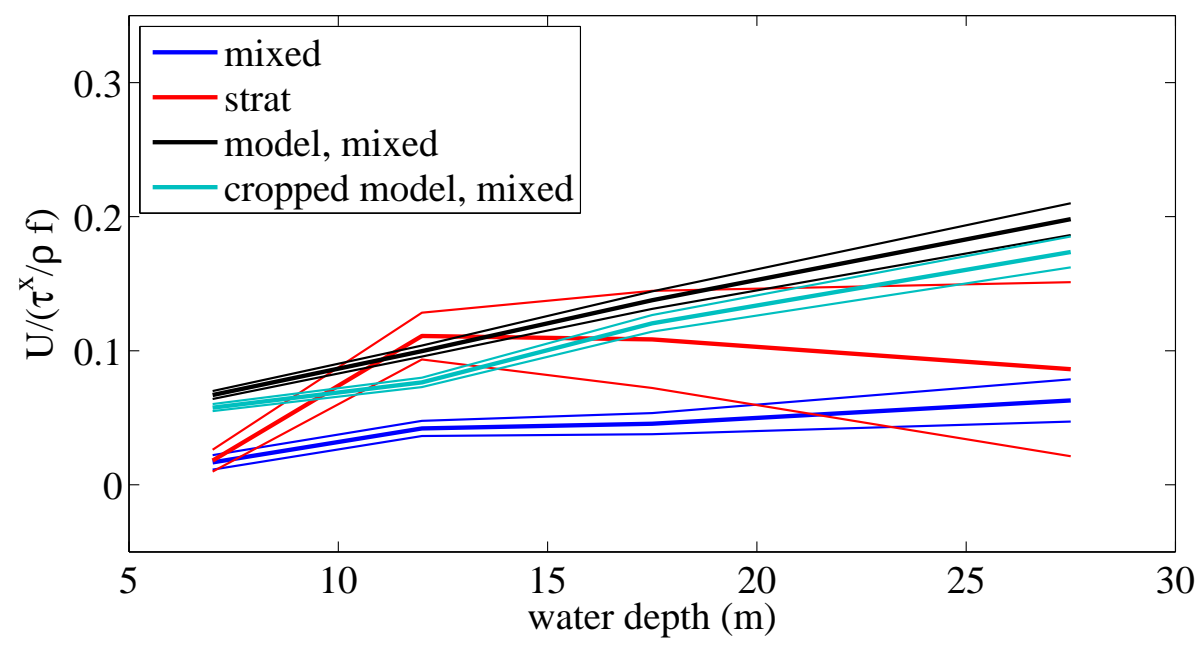

Figure 4-14: $A \sim U /\left(\tau^{s x} / \rho f\right)$ for mixed and stratified conditions, and from 1D unstratified model, using full depth and cutoff at top ADCP bin height. Need to add legend

\section{Strength of stratification}

As for along-shelf wind stress in the second part of section 4.3.2, to evaluate the effect of varying stratification on cross-shelf transport, we use total water column stratification, $\mathrm{N}^{2}$, to make our independent variable in order to better compare our observations to previous works. Figure 4-15 shows fraction of Ekman transport by cross-shelf wind stress as a function of Brunt-Väisälä frequency, $N^{2}=-\left(g / \rho_{0}\right)\left(\partial \sigma_{\theta} / \partial z\right)$, calculated in quintiles of stratification strength, excluding mixed conditions, for each of the four mooring sites. The black line in Figure 4-15 is the transport fraction predicted by Tilburg's (2003) deep water parametrization $U=0.32\left(\tau^{s x} / \rho f\right)\left(1+C_{2} N^{2} / f^{2}\right)^{-1 / 4}$, and empirical constant $C_{2}=2 \times 10^{-4}$.

Here, we find no clear relationship between $U / U_{E k}^{x}$ and $N^{2}$. This results does not match Tilburg's (2003) results of transport by cross-shelf wind stress decreasing for increasing $N^{2}$ in deep water, nor does it align with our expectation, based on the 


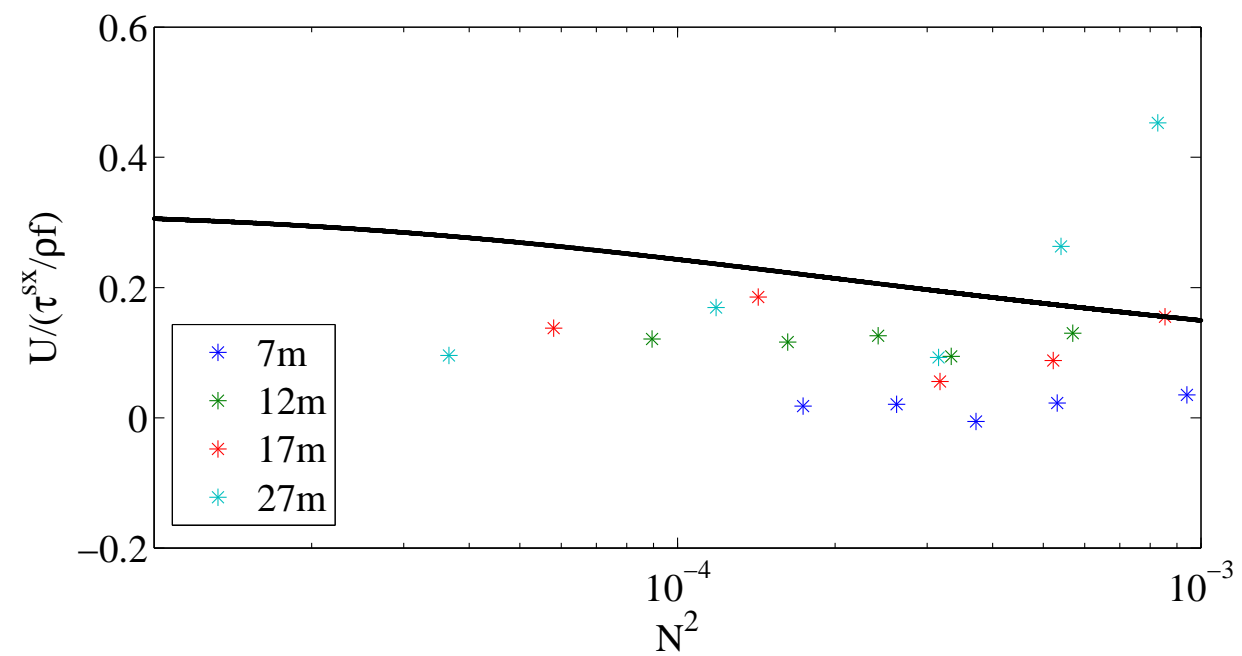

Figure 4-15: $A \sim U /\left(\tau^{s x} / \rho f\right)$ vs $N^{2}$

separation of mixed from stratified conditions in the previous section, of an increase in $U / U_{E k}^{x}$ with increasing $N^{2}$ on the inner shelf where increasing stratification will increasingly reduce mixing. Tilburg did not specifically report on the effect of initial stratification on inner shelf transport, but it appears that his 2D numerical model never created a stratified inner shelf so there is not a direct comparison to be made with these observations. This lack of a clear relationship may be due, at least in part, to the large confidence intervals on our $u / u_{*}^{x}$ and $U_{E k}^{x}$ calculations (not shown, similar to those in Figure 4-13) when we further subdivide stratified conditions into various strengths.

\subsubsection{Asymmetry}

As with the upwelling and downwelling wind stress scenario, in the case of uniform water density, theory predicts a symmetric response to onshore and offshore wind stresses. When the water is stratified, we expect an offshore wind stress to enhance stratification by pulling denser water from deeper, offshore locations into the lower water column and an onshore wind stress to decreasing stratification by forcing lighter fluid under denser. Here we separate times of onshore and offshore wind stress. In the mixed cases, this separation demonstrates how far our field data varies from 
the theoretically symmetric response. In the stratified cases, we observe the effect of existing stratification on the inner shelf by presenting the circulation driven by on- and offshore wind stresses in stratified conditions. Tilburg's model analysis did not demonstrate any asymmetry in transport driven by onshore and offshore wind stresses. However, that analysis was based on mid shelf transport where circulation is confined to the surface mixed layer so there was no cross-shelf buoyancy advection by the cross-shelf circulation.

\section{Velocities}

Figure 4-16 shows the stratified (red) and mixed (blue) response to onshore (4-16(a)) and offshore (4-16(b)) wind stresses. The mixed profiles (blue lines in Figure 4-16(a) and 4-16(b)) show the symmetry expected for uniform density water and are the same to within their $95 \%$ confidence intervals for most of the water depth. Very near the surface the onshore wind drives a stronger velocity then the offshore wind. This difference may be due to our linear treatment of the truly nonlinear combination of along-shelf and cross-shelf wind stresses (see thesis section 5.6).

For onshore wind stress (Figure 4-16(a)), the stratified response is not significantly different from the mixed response at the $95 \%$ level. At the 12, 17, and 27-m sites the stratified responses are not more sheared near the surface than for the mixed cases. For offshore wind stress (Figure 4-16(b)), the stratified profiles are more sheared than the mixed ones with significantly higher (near surface) or lower (near bottom) values than the mixed profiles throughout much of the water column at the 12, 17, and 27-m sites.

The most interesting and obvious difference between offshore and onshore stratified profiles is the general difference in shape. The offshore profiles have a deeper surface region of flow in the direction of the wind stress. At the 17 and $27-\mathrm{m}$ sites, the return flow also appears to have uniform magnitude over several meters of the lower water columns before curving back towards zero approaching the bed. In contrast, the onshore, stratified profiles have a more gradual and uniform shear throughout the 
water column.

The stratified cases for offshore wind stress have lower average stratifications than for onshore wind stress at all four sites and the difference is significant at the 17 and $27-\mathrm{m}$ sites. It is the offshore wind that drives a more sheared circulation so it is not a higher stratification that allows the observed higher shear. One possible explanation is that the higher stratification more fully separates the surface and bottom boundary layers, more often preventing the onshore wind stress from driving circulation throughout the water column. The difference in the stratified response between onshore and offshore wind stresses also may be due to buoyancy advection of the cross-shelf density gradient. South of Martha's Vineyard, water near the coast tends to be lighter than water farther from shore, so the offshore wind stress, while acting on a lower average stratification, is contributing to an increase in stratification by advecting lighter near-shore water over denser water from father offshore. In contrast, the onshore wind stress will advect denser water from offshore over lighter water from nearer to shore.

\section{Transport}

Stratification increases the cross-shelf transport for both on- and offshore wind stresses (Figure 4-17) and both cases reflect the behavior observed in the combined data set in section 4.4 .2 of increasing separation of mixed and stratified conditions with water depth due to an increase in the stratified transport. For offshore wind stress, the stratified transport is larger than the unstratified transport at all water depths and the difference is significant the 12 and 17-m sites. For onshore wind stress, the stratified transport is larger than the unstratified transport at the 12,17 , and $27-\mathrm{m}$ sites, but the differences are not significant at the $95 \%$ confidence level.

Fewings et al. (2008) found higher transport by offshore wind stress in summer than in winter. However, in that analysis, transport by onshore wind stress did not increase and was actually slightly lower than the wintertime transport by the same magnitude onshore wind stresses. This difference is surprising since data for 

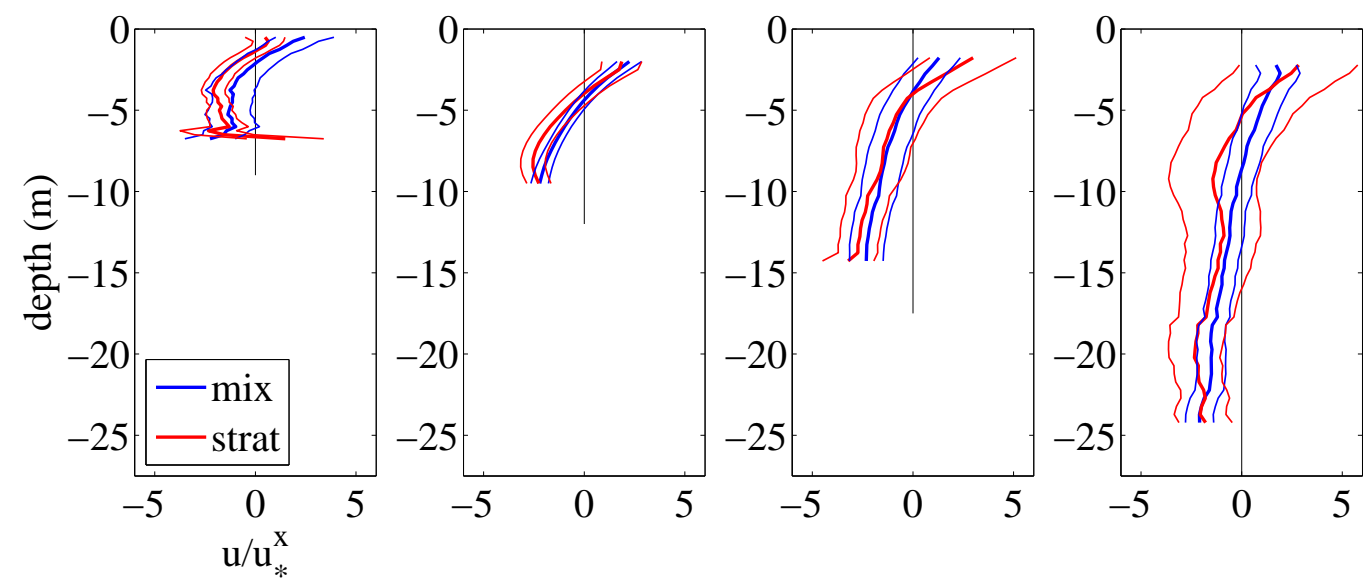

(a) Onshore
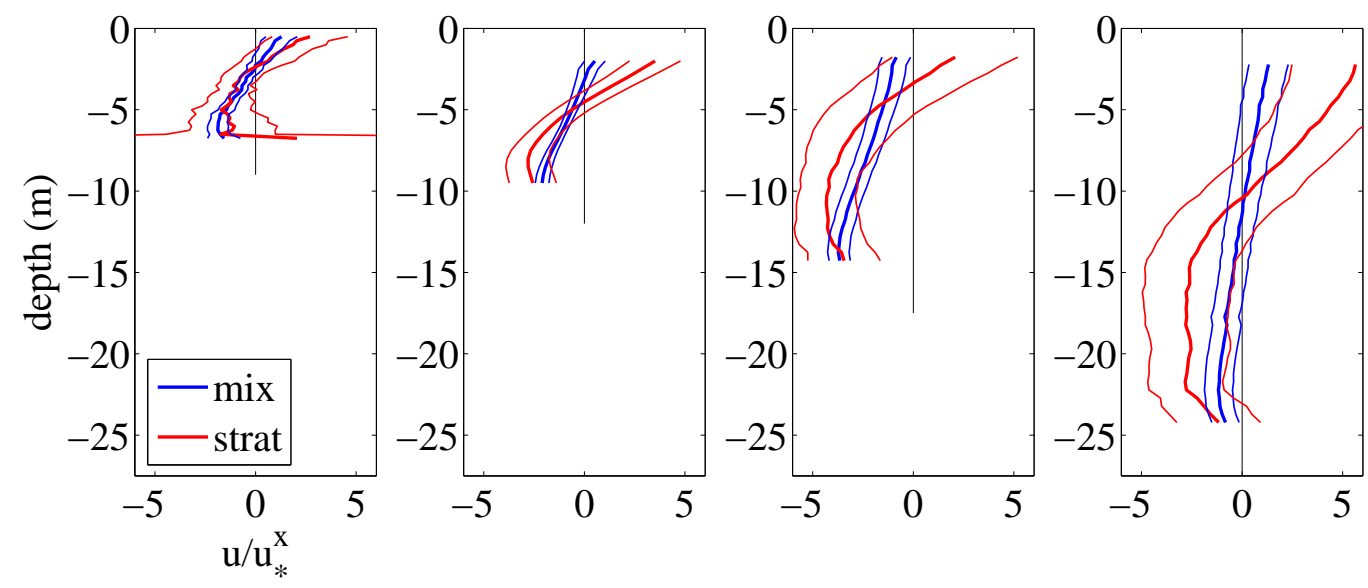

(b) Offshore

Figure 4-16: $a \sim u / \sqrt{\tau^{s x} / \rho}$ mixed and stratified conditions with on- and offshore wind stress

Fewings et al.'s work were collected from the same location as our 12-m site. The discrepancy may be explained by Fewings et al.'s lack of stratification data. While season is generally a good indicator of stratification (see Chapter 2, section 2.2.3), that relationship is strongest at our deepest sites and weakest at the shallowest ones. It is possible that the subset of summertime conditions Fewings et al. used (onshore wind stress with small along-shelf wind stress and small waves) also selected for times that were well mixed. While confidence intervals are wide at all depths, the switch to having higher transport by onshore wind than offshore wind at the $27-\mathrm{m}$ 
site in stratified conditions may indicate the $27 \mathrm{~m}$ is far enough from shore that stratification there is not affected by the overturning circulation on the inner shelf on a synoptic time scale. Away from a coastal boundary, symmetry is expected in the response to on- and offshore wind stresses. Tilburg (2003) found no difference in evolution of circulation or stratification between on- and offshore wind stresses, as Austin and Lentz (2002) did for the up- and downwelling cases. Tilburg's comparison of cross-shelf transport to a linear function of wind stress with a weak dependence on stratification (Equation 25 and Figure 14 in Tilburg, 2003) indicates that none is expected from theory or generated by that model configuration.

In unstratified conditions, a symmetric response to onshore and offshore wind stress is expected, but in these observations onshore wind stress (blue) drives more transport than offshore wind stress (cyan) at 12, 17, and $27 \mathrm{~m}$ depths, with a significant difference at the $12-\mathrm{m}$ site. The observed asymmetry suggests the circulation is either not completely two-dimensional, as we have assumed, or not completely separated from the influence of the along-shelf wind stress.

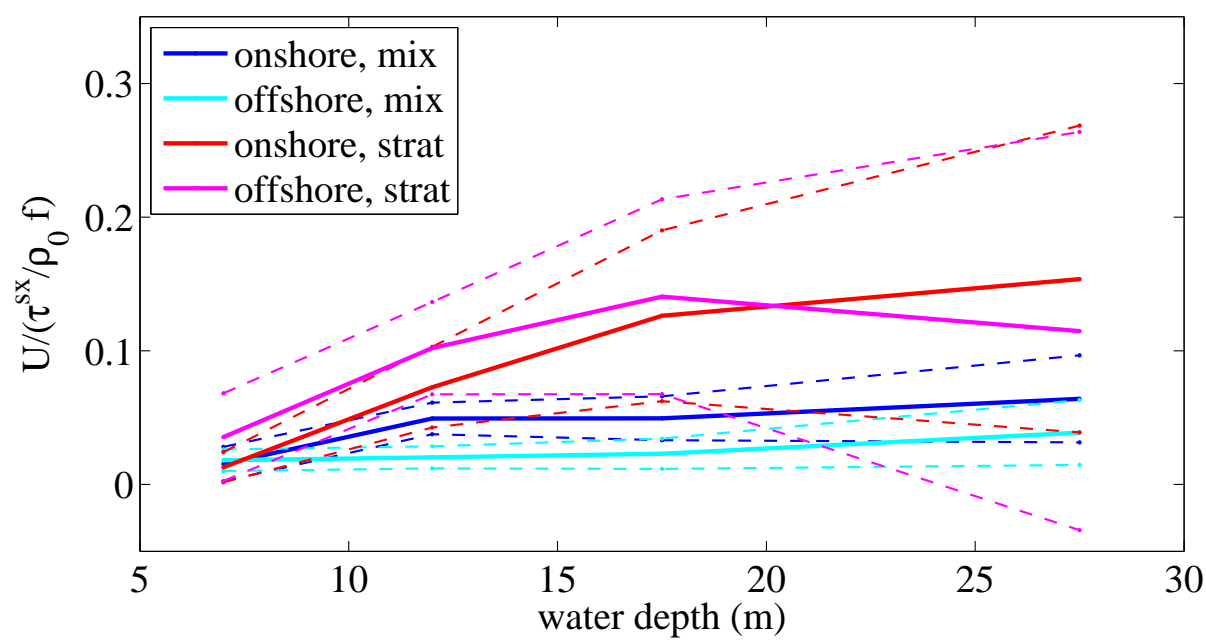

Figure 4-17: $A \sim U /\left(\tau^{s x} / \rho f\right)$ for mixed and stratified conditions with on- and offshore wind stress 


\subsection{D Dynamics}

Our working hypotheses for cross-shelf circulation by along-shelf wind stress (Austin and Lentz, 2002) and cross-shelf wind stress (Tilburg, 2003) are illustrated in previous model results with several notable differences. In each case, the model's incessant wind never affords the newly steepened isopycnals a chance to restratify and new buoyancy is never added so the source for density gradients is only destroyed and never regenerated. These two processes may explain the discrepancies between model behavior and our measurements.

So far in this analysis, we have presented the response to wind forcing as a steady and one-dimensional process by comparing collocated density and velocity measurements and considering each of our four mooring sites separately. Now we explore the spatial and temporal evolution of a wind-driven event to explain the processes that drive the observed responses. The first subsection (4.5.1) outlines the individual contributions of a cross-shelf density gradient, vertical mixing, advection, and surface heat flux could have on the structure of the velocity and density fields. The second subsection (4.5.2) describes how those mechanisms combine on the inner shelf and affect the two-dimensional circulation we understand to result from along-shelf and cross-shelf wind stresses. The third subsection (4.5.3) discusses the significance of two-dimensional processes on a truly three-dimensional inner shelf.

When the water is stratified, we envision a wind stress to drive both vertical mixing and horizontal advection of buoyancy. Wind mixing to the bottom over sloping bathymetry creates a horizontal density gradient as it destroys the vertical one. Once a cross-shelf density gradient is set up, the cross-shelf velocity can advect buoyancy across the shelf while shear in the cross-shelf velocity can enhance or reduce stratification. A cross-shelf density gradient may generate stratification as vertical isopycnals slump into a thermal wind balance. Throughout mixing and slumping events, a surface heat flux can continuously add buoyancy at the surface. We will address the time and spatial scales of each of these processes separately and then in combination. 


\subsubsection{Mechanisms}

\section{Thermal wind balance}

The slumping of a cross-shelf density gradient into a thermal wind balance can create stratification. An unbalanced cross-shelf density gradient will accelerate a vertically sheared cross-shelf flow until the Coriolis acceleration turns the flow to the right. At that point a thermal wind balance is established, with vertical shear in the along-shelf velocity balancing the cross-shelf density gradient. The strength of the cross-shelf density gradient determines how far the isopycnals slump during spin-up and the speed of the final flow, and the time to establish the thermal wind balance scales as $f^{-1}$. If we assume a constant balance between acceleration and the cross-shelf pressure gradient over the spin-up time, we can estimate the final horizontal spread of initially stacked water parcels by twice integrating the momentum equation. By estimating the slumping distance of an initially vertical isopycnal, we can estimate the stratification created by the slumping process.

$$
\frac{\partial u}{\partial t}=\frac{1}{\rho} \frac{\partial P}{\partial x} \quad \text { with } \quad \frac{\partial P}{\partial z}=-\rho g
$$

so for parcels vertically separated by height $h$

$$
\begin{aligned}
\frac{\Delta u}{\Delta t} & =\frac{1}{\rho} \frac{\Delta \rho g h}{\Delta x}=-\frac{g h}{\rho} \frac{\Delta \rho}{\Delta x} \\
\Delta u & =\frac{g h}{\rho} \frac{\Delta \rho}{\Delta x} T \quad \text { with } \quad \Delta t=T \\
\text { then } \quad \Delta X & =\frac{g h}{\rho} \frac{\Delta \rho}{\Delta x} \frac{T^{2}}{2}
\end{aligned}
$$

where $\Delta u$ is difference in the two parcels' cross-shelf velocities, $\Delta X$ is the parcels' cross-shelf separation, and this scaling should hold over spin up period $T=2 \pi / f$. Typical values for these terms are $g=\mathrm{O}(10) \mathrm{m} / \mathrm{s}^{2}, h=\mathrm{O}(10) \mathrm{m}, \rho=\mathrm{O}\left(10^{3}\right) \mathrm{kg} / \mathrm{m}^{3}$, $\Delta \rho / \Delta x=\mathrm{O}\left(10^{-5}\right) \mathrm{kg} / \mathrm{m}^{4}$, and $T=\mathrm{O}\left(10^{5}\right)$ s. With this scaling, we find a slumping isopycnal could move an initially stacked top-bottom water parcel pair roughly $5 \mathrm{~km}$ 
apart in the cross-shelf direction, creating a vertical density difference of $0.05 \mathrm{~kg} / \mathrm{m}^{3}$ over the $10 \mathrm{~m}$ water depth. Coincidentally, $\Delta \sigma_{\theta}=0.05 \mathrm{~kg} / \mathrm{m}^{3}$ is the cutoff we used in the observations to define stratified conditions so any cross-shelf density gradient on the upper end of the typical range of values could potentially create a stratified inner shelf as it slumped into thermal wind balance. For a cross-shelf density gradient, the thermal wind balance is

$$
\frac{\partial v}{\partial z}=-\frac{g}{\rho_{o} f} \frac{\partial \rho}{\partial x}
$$

The spin up period, $2 \pi / f$, is approximately 17 hours at this latitude, but the thermal wind balance holds best on longer time scales in the data. Figure 4-18 shows an evaluation of the thermal wind balance at Martha's Vineyard. vertical shear of alongshelf velocity compared to a prediction for that value based on the cross-shelf density gradient. Data in Figure 4-18 were smoothed with a 2-week low pass filter to remove higher frequency, synoptic scale accelerations from wind-stress driven circulation. The vertical shear was estimated by the slope of a linear fit to the along-shelf velocity profiles. The cross shelf density gradient was estimated by differencing the depthaverage density from one mooring site with an average of the density measurements from the surface down to the matching depth from the next deeper mooring location.

\section{Mixing}

Wind mixing times scales are short for both stratified and unstratified conditions. A typical surface stress $\tau=0.1 \mathrm{~Pa}$ yields a shear velocity, $u_{*}=\sqrt{\tau / \rho}=\mathrm{O}\left(10^{-2}\right)$ $\mathrm{m} / \mathrm{s}$. The unstratified turbulent boundary layer scaling $\delta=\kappa u_{*} / f$ with Von Kármán constant $\kappa=0.4$, is $\mathrm{O}(100) \mathrm{m}$, which is greater than our water depth. Instead, mixing time will be limited by our water depth, $\mathrm{d}=\mathrm{O}(10) \mathrm{m}$, during unstratified conditions. In stratified conditions, the mixed layer thickness scales as $\delta=u_{*} / \sqrt{N f}$ as described by Pollard et al. (1973). A typical value of Brunt - Väisälä frequency is $\mathrm{N}=10^{-2}$ $\mathrm{s}^{-1}$ yields a boundary layer thickness scalings $\delta=u_{*} / \sqrt{N f}=\mathrm{O}(10) \mathrm{m}$. We find a mixing time scale, $\delta / u_{*}$, to be $\mathrm{O}\left(10^{3}\right)$ s or about 20 minutes. This short time scale 


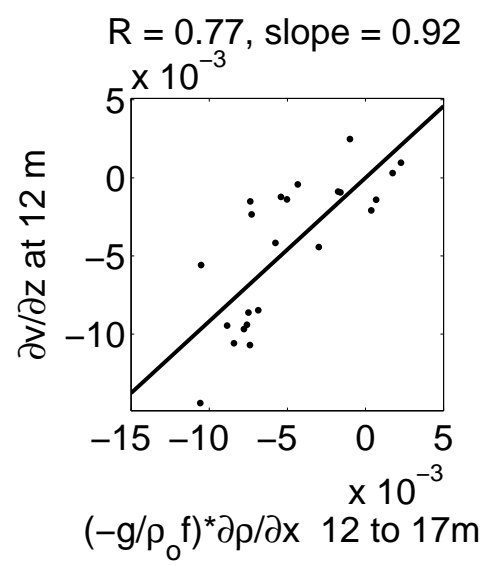

(a) $12 \mathrm{~m}$

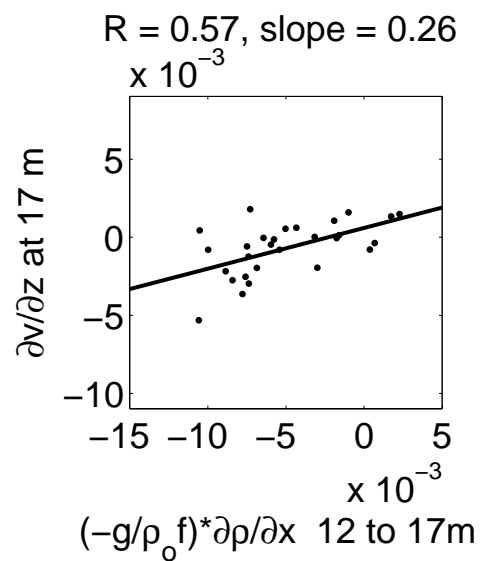

(b) $17 \mathrm{~m}$

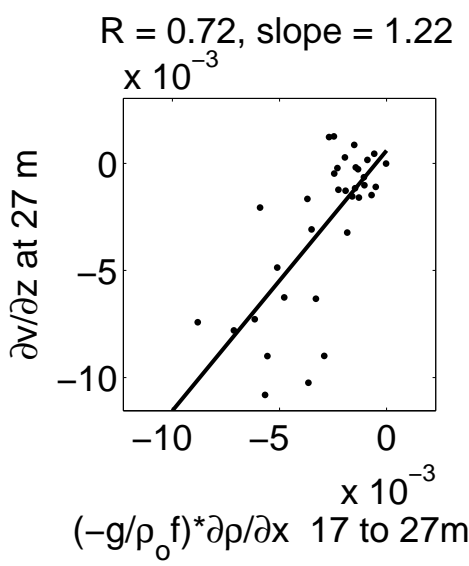

(c) $27 \mathrm{~m}$

Figure 4-18: Testing thermal wind balance between shear at 12, 17, and $27 \mathrm{~m}$ ADCPs with density difference between pairs of adjacent moorings. Data are filtered and subsampled with a two-week low pass filter.

for adjustment confirms that mixing should reach a nearly steady state in the time between field measurements (also 20 mins).

Our observations match both qualitatively and quantitatively with these predictions for mixed layer thickness. The 7 and $12-\mathrm{m}$ sites are frequently mixed and restratified during the summer, while the 17 and 27 -m sites remain stratified throughout the summer season, suggesting that the mixed layer thickness our summer conditions tend towards is somewhere between 12 and $17 \mathrm{~m}$ thick. When we compare predictions of mixed layer thickness to stratification data in Figure 4-19, we find the expected trend of mixed layer thickness increasing with wind stress. The wind stress appears to set the minimum mixed layer thickness. This bias makes sense because as wind stress increases, we expect the mixed layer growth to keep adjusting faster than we are (sub)sampling. As wind stress decreases, the water column does not instantly restratify without the addition of buoyancy from surface heating or horizontal advection, which respond over longer time scales. Surface cooling and wave mixing could also create or thicken a surface mixed layer without the need for mixing by surface wind stress. 


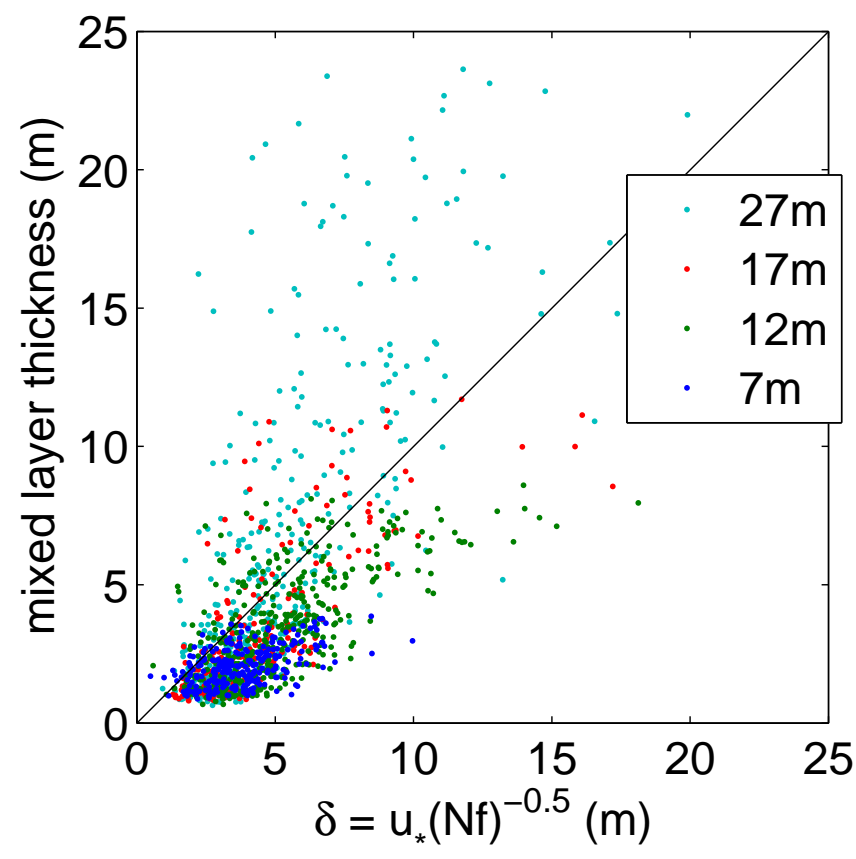

Figure 4-19: Testing $\delta_{E k}=u_{*} / \sqrt{N f}$ as scale for mixed layer thickness

\section{Advection}

Where there is a cross-shelf density gradient, cross-shelf circulation can advect buoyancy across the shelf and feedback on the stratification. We estimate how far a density front might be advected during a typical wind event and how much buoyancy advection could increase stratification in the case of upwelling or offshore wind stress.

In the Austin and Lentz (2002) model, a density front is moved continuously offshore or onshore by upwelling or downwelling wind stress, respectively. When the model was initialized with strong stratification between two constant-density layers, in both upwelling and downwelling cases the region onshore of the density front filled up with unstratified water. We have noticed that our deeper mooring sites, particularly the 27-m site, remain stratified throughout the summer season so we ask how far a front might be advected during a summertime wind event and if it is reasonable to expect the 27-m site to remain outside the well-mixed region. We will not separate the up and downwelling responses at this time. In a typical summer wind event with 
$\tau=0.1 \mathrm{~Pa}$, for $\mathrm{T}=$ one day or $\mathrm{O}\left(10^{5}\right) \mathrm{s}$, the cross-sectional area flushed by the Ekman transport during an event is expected to scale as $\tau / \rho f \times T$. This transport could flush out a nearshore triangular cross-sectional area of $1 / 2 \times d \times \Delta x$ for depth $d$ and distance to shore, $\Delta x=d / \alpha$, where $\alpha$, bottom slope, is $\mathrm{O}\left(10^{-3}\right)$ at our site. Combining these scalings, we estimate the depth to which an up- or downwelling front would reach to be

$$
d=\sqrt{\frac{2 \alpha T \tau}{\rho f}} \approx 14 \mathrm{~m}
$$

Thus for a moderate wind stress, we do not expect an up or downwelling front to reach the 27-m mooring within a day. Flushing the volume onshore of the 27-m site would take four times the volume and hence four times as long as to fill the shelf inside of $14 \mathrm{~m}$ depth. Wind stresses south of Martha's Vineyard are dominated by synoptic scale events that are usually less than four days in duration. In sections 4.3.2 and 4.4.2 we observed cross-shelf transport by along- and cross-shelf wind stresses in stratified conditions to be less than $25 \%$ of the theoretical Ekman transport value so if we scale down the estimate of d accordingly, with $U=U_{E k} / 4$ we find $d \approx 7 \mathrm{~m}$. Making the same calculation with our lower, observed transport fraction makes the idea of and up- or downwelling front reaching the 27-m site highly unlikely.

The second question we ask about cross-shelf advection is how much buoyancy is advected in a typical wind event? Even if these conditions do not have a clear representation in numerical models run by Austin and Lentz (2002) or Tilburg (2003), we know our field site usually has a positive cross-shelf density gradient (lighter water near the coast, denser offshore) and that gradient is larger in summertime (Figure 2-10), when stratification is common. For the observed transport response, $U=U_{E k} / 4$, distributed over a mixed later thickness $\delta=10 \mathrm{~m}$ for $\tau=0.1 \mathrm{~Pa}$ for one day, we estimate a given outcropping isopycnal can move $\Delta x=U \times T / \delta=2.5 \mathrm{~km}$. Summertime $\partial \rho / \partial x$ is of $\mathrm{O}\left(10^{-5}\right.$ to $\left.10^{-4}\right) \mathrm{kg} / \mathrm{m}^{4}$ (table 2.3). Using the smaller value, advection of this typical gradient could create $2.5 \times 10^{-2} \mathrm{~kg} / \mathrm{m}^{4}$ of vertical density difference in a day. The resulting increase in stratification would be, $N^{2}=2.5 \times 10^{-4}$ 
$\mathrm{s}^{-2}$, a measurable and dynamically significant change. The larger cross-shelf density gradient value is typical for the span between our 7 and $12-\mathrm{m}$ moorings while the lower value is representative of the gradients spanning 12 to $27-\mathrm{m}$ sites. Finding this larger gradient in shallower water supports our earlier hypothesis that the crossshelf density gradients are generated when mixing reaches the bottom in previously stratified location of varying total water depth. The surface mixed layers at the 17 and $27-\mathrm{m}$ sites do not reach the bottom in summertime so have similar density values at a given depth below the surface.

\section{Surface heat flux}

Surface heat flux, $Q$, varies on two major times scales, daily and annual cycles. The daily cycle is large but similar in period to the 24 and 24.84 hour tidal constituents so we filter it out of the density and velocity data and create a daily-average value of surface heat flux to use for this analysis. The seasonal cycle heats the shelf over the summer months as shown in Chapter 2, Figure 2-3 and described in detail by Fewings and Lentz (2011). Daily averages (Figure 2-5), further modulated from the seasonal cycle by weather conditions, are around $200 \mathrm{~W} / \mathrm{m}^{2}$ in the summer. The heat capacity, $c_{p}$, of seawater is about $4 \times 10^{3} \mathrm{~J} / \mathrm{kg}{ }^{\circ} \mathrm{C}$ so in a day, this heat flux can raise the water temperature by about $0.5{ }^{\circ} \mathrm{C}$ over $10 \mathrm{~m}$ water depth. For mixing through the full water depth over a bathymetry slope of $\alpha=10^{-3}$, this surface heat flux would create a cross-shelf density gradient $\mathrm{O}\left(10^{-5}\right) \mathrm{kg} / \mathrm{m}^{4}$ in $10 \mathrm{~m}$ water depth. If instead the heat flux was mixed through only the upper half of the water column, it would instead create a stratification $\mathrm{N}^{2}$ of $\mathrm{O}\left(10^{-4}\right) \mathrm{s}^{-2}$, a small but measurable value at Martha's Vineyard.

\subsubsection{Comparing and combining forcing mechanisms}

Mixing, heating, advection by wind driven flows, and advection by pressure gradient driven flows (slumping) are the four mechanisms discussed above that combine to drive the transport and stratification patterns we observed in sections 4.3 and 4.4. 
Mixing works on a short, 20 minutes, time scale. Slumping of isopycnals and spinup of a thermal wind balance evolve over an inertial period of 17 hours. Advection by wind driven flow occurs over synoptic time scales of a day or a few days. The component of surface heat flux we address here varies over daily and synoptic time scales. The truly nonsteady and nonlinear nature of these processes makes them difficult to separate in the data so we consider their spatial and temporal scales together with evidence from our observations to decide which balances are likely dominant and testable with a numerical model.

Here, we keep in mind that density is a dynamic tracer, simultaneously affecting and being transported by the circulation. The speed with which mixing occurs may prevent the other mechanisms from generating the stratification that would be expected from considering each alone. Surface heat flux alone clearly increases stratification and the summertime values are high enough to increase stratification on a daily time scale, but if mixing is too strong for vertical density gradients to grow, the surface buoyancy flux will instead contribute to the cross shelf density gradient wherever mixing reaches the sloping bottom. A cross-shelf density gradient can lead to stratification by slumping or advecting of isopycnals, but these processes would also be inhibited by strong mixing. After wind has created a mixed layer and steepened isopycnals to vertical, any small increase in stratification may quickly be mixed away by the still-high eddy viscosity before enough stratification is regenerated to inhibit mixing. The short time scale for mixing compared to that for advection and surface heating may prevent the positive feedback we expect between upwelling or offshore wind stress and stratification. Quiescent periods during the deceleration of wind-driven flow may provide the chance for buoyancy to be advected without instant remixing. Any coincident surface heat flux would further enhance the restratification process. 


\section{Along-shelf wind}

For along-shelf wind stress, our observations generally agree with previous observational (Lentz, 2001; Kirincich et al., 2005) and modeling (Weisberg et al., 2001; Austin and Lentz, 2002) results. We find transport contained in the surface mixed layer and note that our deeper sites tend to remain stratified throughout wind events in the summer season (see Chapter 2). Shallower regions become mixed and along-shelf wind stress becomes ineffective at driving cross-shelf circulation there. On a shelf with with a positive cross-shelf density gradient (lighter water near shore, denser far from the coast), upwelling circulation should enhance stratification, while downwelling circulation should decrease stratification. However, our stratification data shows a surprising lack of sensitivity to wind direction and there is no clear difference in the relationship between wind stress and stratification between upwelling and downwelling condition. When the 27-m site is stratified, there is not the expected behavior of upwelling wind stresses consistently being more likely to maintain stratification at the shallower sites than downwelling wind stresses.

While the velocity profiles in Figure 4-10 are shaped differently for upwelling and downwelling wind stress, our four fixed measurement locations may not capture a key feature of differences between upwelling and downwelling events - the spatial and temporal evolution of a nearly vertical density front. On the stratified side, there is little difference between upwelling and downwelling events and on the mixed side, we no longer consider our measurements to be part of a stratified event.

A second inconsistency we noted earlier is that while our estimates of mixed layer thickness indicate our deeper site should have well-separated surface and bottom boundary layers in stratified conditions, we see a continued increase in Ekman transport fraction with both increasing water depth and stratification (Figure 4-9). While a day is longer than the inertial period we expect Ekman transport to require for spinup or spin-down, the temporal evolution of circulation and transport is still blurred by our filtering and subsampling process so the profiles we examine may not represent the magnitude of a fully developed Ekman spiral. Since the mixing process is 
not reversible, including both accelerating and decelerating times does not necessarily have a net zero effect on the smoothed result. Of the various wind and stratification conditions we've considered, the velocity profile for stratified upwelling conditions at the 27-m site (Figure 4-10(a)) shows the only hint of a surface and bottom boundary layer separated by a less sheared interior. Separated boundary layers are also suggest by an Ekman transport fraction that reaches 1 for strong stratification at the 27-m site (Figure 4-9).

\section{Cross-shelf wind}

For cross-shelf wind stress, there is surface mixing by wind and a near-surface transport in the direction of wind stress that is either balanced by the lower portion of an Ekman spiral in deep water or a pressure gradient driven return flow in water less than a boundary layer thickness in depth. On a shelf with with a positive cross-shelf density gradient (lighter water near shore, denser far from the coast), offshore wind should enhance stratification and decrease the cross-shelf density gradient, while onshore wind should decrease stratification and increase the cross-shelf density gradient. We would expect then, to measure higher stratification during times of offshore wind stress, but this is not the case. The stratification is slightly higher, on average, during onshore than offshore wind stress conditions, indicating that advection by the crossshelf wind stress is not the mechanism generating the stratification. The directional effect may be partly obfuscated by the correlation of onshore and upwelling favorable wind stresses in the summertime, when the wind usually blows towards the northeast. These two components of the wind tend to move the surface water in opposite crossshelf directions. The lack of a clear difference in the relationship between wind stress and stratification for onshore and offshore wind directions is surprising in light of the larger increase in circulation and transport with stratification by offshore winds, as described in section 4.4.3.

In stratified water deeper than a boundary layer thickness, we expect a full Ekman spiral to developed with cross-shelf velocities in the direction of the wind near the 
surface and a return flow directly beneath it, all within the surface boundary layer. At the 7,12, and 17-m sites, most of our stratified profiles for onshore, offshore, and combined wind directions (Figures 4-12, 4-16) indicate only a wind stress - pressure gradient balance with return flow distributed throughout the water column below the first zero crossing of velocity. However, in Figure 4-13, profiles from the 17 and 27-m sites indicate that the lower portion of the circulation has a more complicated structure than a smoothly curved pressure gradient driven return. At $27 \mathrm{~m}$, the near surface portions of the three profiles show a smaller surface velocity and higher first zero crossing for a thinner surface mixed layer; this is the trend we would expect from the wind-parallel component of an Ekman spiral limited by mixed layer thickness.

One discrepancy between our observations and Tilburg's model remains. Tilburg's model predicts all circulation to be contained in the surface mixed layer and our observations clearly show that it is not, so we still ask how is circulation driven in the stratified part of the water column or how is stratification maintained in the winddriven flow? Advection, slumping, and surface heat flux all can increase stratification of the inner shelf so we will look to an implementation of ROMS in Chapter 5 to determine which of these mechanisms is most responsible for creating a stratified inner shelf.

\section{Comparing transport by both wind components}

Here we compare the transport driven by along-shelf and cross-shelf wind stresses in mixed and stratified conditions to compare their relative strengths at difference locations across the inner shelf. In mixed conditions (Figure 4-20(a)), the cross-shelf wind stress drives a larger cross-shelf transport than the along-shelf wind stress at all four mooring sites. This result agrees with the observations from $12 \mathrm{~m}$ depth, and 1D numerical modeling results from Fewings et al. (2008). While that 1D model showed along-shelf wind stress approaching the effectiveness of cross-shelf wind stress near $30 \mathrm{~m}$ water depth, that model (the same as the 1D model used in various parts of this chapter) does not include mixing from any sources besides the wind-driven flow. 
In the field, bottom friction from tidal velocities increases the mixing throughout the water column. The higher mixing allows momentum from the surface winds to reach the bottom faster, decreasing the effectiveness of along-shelf winds at a particular water depth, and moving the outer edge of the inner shelf to a deeper location than predicted by the simple model. We notice that the transport fraction by cross-shelf wind stress increases with water depth. This increase is expected because on the inner shelf, where the water depth is less than a boundary layer thickness, we expect the transport by cross-shelf wind stress to scale like $u_{*} h$, which is derived from the classic log layer velocity solution (e.g., Flierl and Ferrari, 2006), rather than the deep water, full boundary layer transport scale of $\tau / \rho f$.

When there is stratification (Figure 4-20(b)), the cross-shelf wind stress drives a larger cross-shelf transport at the 7 and $12-\mathrm{m}$ sites. We speculate that the stratification allows for greater shear in the velocity profiles, but momentum is still transferred from surface to bottom increasing the efficiency of the cross-shelf wind stress / pressure gradient balance. At the 17 and $27-\mathrm{m}$ sites, the along-shelf wind is dominant. This change indicates the surface and bottom boundary layers are more fully separated at the deeper sites, allowing the along-shelf wind to drive a greater fraction of full Ekman transport.

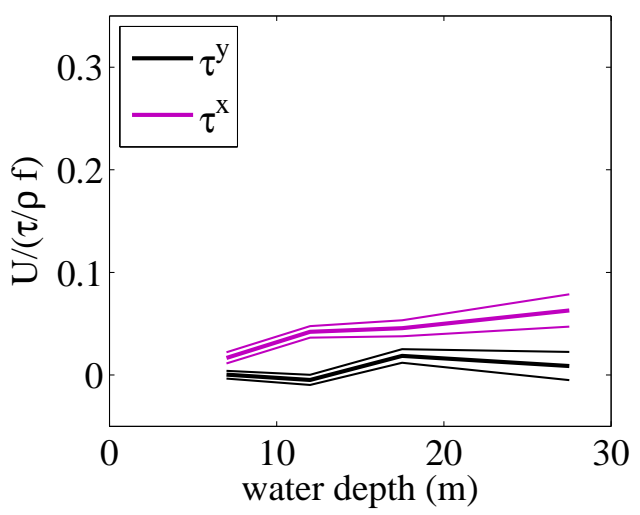

(a) Mixed

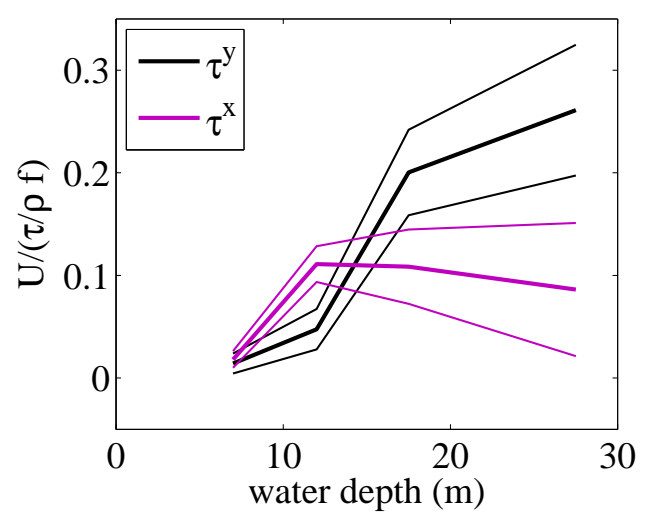

(b) Stratified

Figure 4-20: Transport fraction driven by along-shelf (black) and cross-shelf (purple) wind stresses in mixed (a) and stratified (b) conditions. 


\subsubsection{D analyses on a $3 \mathrm{D}$ inner shelf}

The analyses in this chapter are based on the assumption of along-shelf uniformity. This assumption, combined with the very realistic no flow through the coast constraint, is what allows us to take $\bar{u}=0$ as part of the solution to the steady, shallow water equations. The inner shelf at Martha's Vineyard has smooth, nearly east-west isobaths for most of the width of Martha's Vineyard, but the topography is complicated by varying bed roughness (Goff et al., 2005) and the channel east of the island, between Martha's Vineyard and Nantucket (Ganju et al., 2011). Here we demonstrate the significance of the two-dimensional, wind-driven circulation relative to other sources of cross-shelf transport.

\section{Depth-average flow}

The time-mean depth-average subtidal cross-shelf velocities at all four SWWIM sites are $1.0,0.98,1.7$, and $-0.19 \mathrm{~cm} / \mathrm{s}$ at the $7,12,17$, and $27-\mathrm{m}$ sites with standard deviations of $2.1,1.3,2.1$, and $1.8 \mathrm{~cm} / \mathrm{s}$. At the $12 \mathrm{~m}$ deep MVCO node, Lentz et al. (2008) found that, in winter, the depth-average cross-shelf flow was almost entirely explained by the wave driven undertow, consistent with a two-dimensional (along-shore uniform) circulation at our field site, and Fewings et al. (2008) found that when waves were small at MVCO, the time-mean, depth-average cross-shelf velocity was nearly zero. For the four SWWIM sites, wave-driven circulation in mixed and stratified conditions are indicated by the blue lines in third column of all figures in Appendix 4.A. The wave driven flow is of similar size to the wind-driven circulation, but since Lentz et al. (2008) showed these Eulerian velocities to be equal and opposite to the Lagrangian Stokes drift, the wave undertow may not drive any particle transport. The red lines in those same figures indicate the residual circulation associated with the waves after estimates of the wave undertow were removed from the velocity data. Using the velocity data with wave undertow removed, the remaining time-mean depth-average subtidal cross-shelf velocities at all four SWWIM sites are $-0.8,0.3,1.3$, and $-0.3 \mathrm{~cm} / \mathrm{s}$ at the $7,12,17$, and $27-\mathrm{m}$ sites with standard deviations 
of $1.1,0.9,2.0$, and $1.8 \mathrm{~cm} / \mathrm{s}$. Means are lower in winter than summer with the biggest difference at the $12-\mathrm{m}$ site, where the wintertime mean $(0.16 \mathrm{~cm} / \mathrm{s}$, std 0.84 $\mathrm{cm} / \mathrm{s})$ was similar to the value $(0.1 \mathrm{~cm} / \mathrm{s}$ mean, $0.8 \mathrm{~cm} / \mathrm{s} \mathrm{std})$ reported by Lentz et al. (2008), and the summer time mean was three times that size $(0.50 \mathrm{~cm} / \mathrm{s}$ mean, std $0.088 \mathrm{~cm} / \mathrm{s})$. The larger $(\sim 2 \mathrm{~cm} / \mathrm{s})$ standard deviations of the residual circulation at the 17 and $27-\mathrm{m}$ sites indicate that the wave undertow does not account for the depth-averaged flow as well at the deeper sites as the shallower ones $(\sim 1 \mathrm{~cm} / \mathrm{s} \mathrm{std})$.

In their recent review paper, Lentz and Fewings (2011) used a scaling of the depth-averaged continuity equation to show that on the inner shelf, fluctuations in the depth-averaged cross-shelf velocity were likely due to along-shelf variation in forcing or bathymetry, and that this scaling is the dominant one for $\bar{u}$ in water depth greater than 10-20 m, depending on the specific waves and bathymetry. The standard deviations of the depth-averaged velocity are between $1-2 \mathrm{~cm} / \mathrm{s}$ at all four SWWIM sites. This scale for fluctuating cross-shelf velocity is smaller than, though the same order of magnitude as, the wind-driven circulation, suggesting that along-shelf nonuniformities are neither the dominant nor a negligible source of cross-shelf transport at Martha's Vineyard.

\section{Regression slope profiles}

In the regressions on measured velocity profiles described in section 4.2 .4 , the depthaverage values were not removed from the data so that any non-zero depth-average circulation driven by wind would be evident in the regression slope profiles presented in sections 4.3.1, 4.3.3, 4.4.1, and 4.4.3. This is in contrast to when depth-average values were removed to calculate transport. A regression slope profile with a non-zero depth average indicates that the wind forcing is responsible for some spatially varying circulation, either due to spatial variation in the wind forcing or wind forcing acting on nonuniform along-shelf bathymetry. Assuming the perfect linear relationship described by the regressions, a non-zero depth-average value of the regression slopes would contribute to the time-mean circulation as a multiplier of the time-mean of the 
forcing time series $\left(u_{*}^{x}\right.$ or $\left.u_{*}^{y}\right)$. Most of the regression slope profiles used in this chapter have near zero depth-averages, with the exception of the 17-m site in mixed conditions which appears to have a net onshore flow driven by downwelling and offshore wind stresses.

The final term in the velocity regressions of section 4.2 .4 is the constant term, which has not been discussed until now. Values for this term for mixed and stratified conditions at all four sites are shown in the last column of all figures in Appendix 4.A. Note that the horizontal axis limits of these figures are 100 times smaller than for the other three terms of the regression. Since this term is not a ratio to any velocity scale, it directly represents a cross-shelf velocity, in units of $\mathrm{m} / \mathrm{s}$. Typical values of the velocity scales, $u_{*}$ and $u_{S t}$, are $0.01 \mathrm{~m} / \mathrm{s}$ so a value of 0.01 for the constant term represents a similar strength circulation to a value of 1 for the other terms.

The depth-average value of this constant term makes up the remaining portion of $\bar{u}$ that is not accounted for by time-means of the depth-averages of the wind terms in the regression, $<\bar{a} u_{*}^{x}+\bar{b} u_{*}^{y}>$. The vertical structure of this term, however, could also lead to cross-shelf transport without violating any no flow through the coast assumptions in the same way that transport by wind-driven circulation does: above the first zero crossing of the regression profile. These transports turn out to be small compared to the wind-driven circulation. Using $0.01 \mathrm{~m} / \mathrm{s}$ as a typical velocity scale for the wind and wave terms, which leads to an Ekman transport, $U_{E k} \sim 1 \mathrm{~m}^{2} / \mathrm{s}$, we find that in stratified conditions, the constant terms drives a transport of 1-3\% of the Ekman transport, and is smaller than but the same order of magnitude as the wind-driven transport. In stratified conditions, the constant cross-shelf transport is slightly larger, ranging from 2-6\% of the Ekman transport at the 7 - 27-m sites. However, due to the increase in wind-driven transport in stratified conditions, the constant transport is an order of magnitude smaller than the wind-driven transport at all water depths when the water is stratified. These mean cross-shelf circulations not associated with wind or waves are likely tidally rectified flow (Ganju et al., 2011), similar to the velocity profiles observed by Fewings et al. (2008) in small wind, small 
wave conditions.

\section{Spatial structure}

A recent numerical modeling study of the Martha's Vineyard region (Ganju et al., 2011) used three-dimensional realistic bathymetry and nested spatial grids to demonstrate that that, with $40 \mathrm{~m}$ grid resolution, the model can reasonably capture the depth-averaged mean circulation observed at the SWWIM moorings and the surface velocities observed by a nearby HF radar sensor. That model, run for unstratified conditions with realistic tidal and synoptic forcing for September 2009, confirms the radar observations of a spatially complex mean circulation featuring a large counterclockwise gyre south of Martha's Vineyard that is largely created by tidally rectified flow. The center of the gyre is located a few kilometers east of the SWWIM array around the 18-m isobath. The SWWIM observations are used to confirm the model's success and the model results may explain the small observed offshore mean flow not explained by wave undertow. More importantly, Ganju et al.'s (2011) model demonstrates a possible mechanism for cross-shelf transport that is not captured by the SWWIM observations. A few kilometers east of the SWWIM array, the model shows a $1 \mathrm{~km}$ wide along-shelf region with depth-average onshore flow of $5-10 \mathrm{~cm} / \mathrm{s}$ extending from the 20 to $12-\mathrm{m}$ isobath. A $10 \mathrm{~cm} / \mathrm{s}$ velocity over $17 \mathrm{~m}$ water depth (choosing one of our mooring depths) is a cross-shelf transport of $1.7 \mathrm{~m}^{2} / \mathrm{s}$ or $1.7 \mathrm{U}_{E k}$ for a typical $0.1 \mathrm{~Pa}$ wind stress. While $1.7 \mathrm{U}_{E k}$ is 10 times the wind-driven transport observed at the 17-m mooring in stratified conditions and 20 times the transport during mixed conditions, the Martha's Vineyard inner shelf is close to $30 \mathrm{~km}$ wide. If the time-varying, wind-driven transport is uniform across the shelf, the scaling suggests both the spatially uniform, time-varying, wind-driven circulation and the time-mean, spatially varying, tidally rectified flow will make similar contributions to the total cross-shelf transport. 


\subsection{Summary}

Cross-shelf transport across the inner shelf is important to connect the the coastline and surfzone to the midshelf and beyond. Along-shelf wind stress has long been understood to be the mechanism for cross-shelf transport on the midshelf. However, along-shelf winds are poor at driving transport across the inner shelf. Recently, cross-shelf wind stress has been demonstrated to be a more effective mechanism for cross-shelf transport in the inner shelf, but very few measurements have been made of this phenomenon. Here, three years of measurements from four mooring sites across the inner shelf south of Martha's Vineyard, MA are used to describe the effect of stratification on wind-driven cross-shelf circulation and transport by along- and cross-shelf wind stresses. For both along- and cross- shelf wind stresses, this data set allows us to describe the vertical structure of the cross-shelf circulation, its variation with onshore or offshore directed surface flow, and its response to stratification.

Along-shelf wind stress drives near zero cross-shelf transport during unstratified conditions. Stratification dramatically increases shear in cross-shelf circulation at all water depths and the thickness of the surface mixed layer clearly limits the depth of the first zero crossing of the velocity. Cross-shelf transport increases in the presence of stratification and the fraction of Ekman transport increases with increasing stratification. These increases in shear and transport with stratification also grow with water depth, indicating along-shelf wind stress has a greater influence on the cross-shelf circulation over the midshelf rather than over the inner shelf. Circulation and transport by upwelling and downwelling wind stresses are all increased by stratification but the increase from mixed to stratified conditions is larger in the upwelling case.

Cross-shelf wind stress drives cross-shelf circulation and transport on the inner shelf. In unstratified conditions, we measure cross-shelf transport driven by crossshelf wind stress to be one third of that predicted by a $1 \mathrm{D}$ numerical model. Stratification increases vertical shear in cross-shelf circulation, and the thickness of the 
surface mixed layer appears to limit the depth of the first zero crossing of the velocity. Despite the lack of consistent vertical structure of velocity relative to the mixed layer thickness, it is notable that the circulation is not confined to the surface mixed layer for any water depths. Cross-shelf transport also increases in the presence of stratification but is not significantly correlated with the strength of the stratification. These increases in shear and transport with stratification appear to diminish, along with our confidence in these calculations, at the $27-\mathrm{m}$ site, suggesting that at this water depth, cross-shelf wind is not as strong an influence on the cross-shelf circulation as it was in shallower water. We find circulation by offshore wind stress to be more strongly sheared by stratification than circulation by onshore wind stress. The increase in transport from mixed to stratified conditions is also larger for offshore than onshore wind stress.

There are, of course, particularities of our field site we have ignored in our various simplifying assumptions. However, at this site, wind-driven cross-shelf circulation is large compared to the mean depth-averaged, cross-shelf flow and is a similar size to the vertically varying component of the mean cross-shelf circulation so wind forcing will play a substantial role in the cross-shelf circulation near Martha's Vineyard. Here, we isolated the local, stratified response to cross- and along-shelf wind forcing. In stratified conditions, the cross-shelf wind stress drives a larger circulation at 7 and 12 m water depth, while the along-shelf wind stress drives stronger circulation at 17 and $27 \mathrm{~m}$ (Figure 4-20). In unstratified conditions, the cross-shelf wind drives stronger circulation at all four water depths. In addition to their application at this specific field site, these results further the understanding of the two-dimensional features of circulation that are common to many inner shelves, and aid in predicting transport of suspended material across the inner shelf. 


\section{A Appendix: Regressions with and without wave term}

In section 4.2.4, Figure 4-5 shows profiles of regression coefficients from three regressions on velocity from mixed conditions at the 17-m site. Here, profiles from the same three regression methods are presented for all four measurement sites $(7,12,17$, and 27-m depth) for mixed and stratified conditions.

Blue lines use velocity that includes wave undertow and is fit with equation $4.2 \mathrm{~d}$, which includes a wave-forced term. Green lines use velocity that has had estimates of wave undertow subtracted, fit with equation $4.2 \mathrm{a}$, which does not includes a waveforced term. Red lines use velocity that has had estimates of wave undertow subtracted, fit with equation $4.2 \mathrm{a}$, which again includes a wave-forced term.

The first columns of Figures 4-21 to 4-24 show the regression slopes for the crossshelf wind term for all three regression methods. The second columns of Figures 4-21 to 4-24 show the regression slopes for the along-shelf wind term for all three regression methods. The third columns of Figures 4-21 to 4-24 show the regression slope for the wave undertow term for the two regression methods that include it. The fourth

columns of Figures 4-21 to 4-24 show the constant term of the regression for all three regression methods. Note that the horizontal axis limits are 100 times smaller for this final term. 

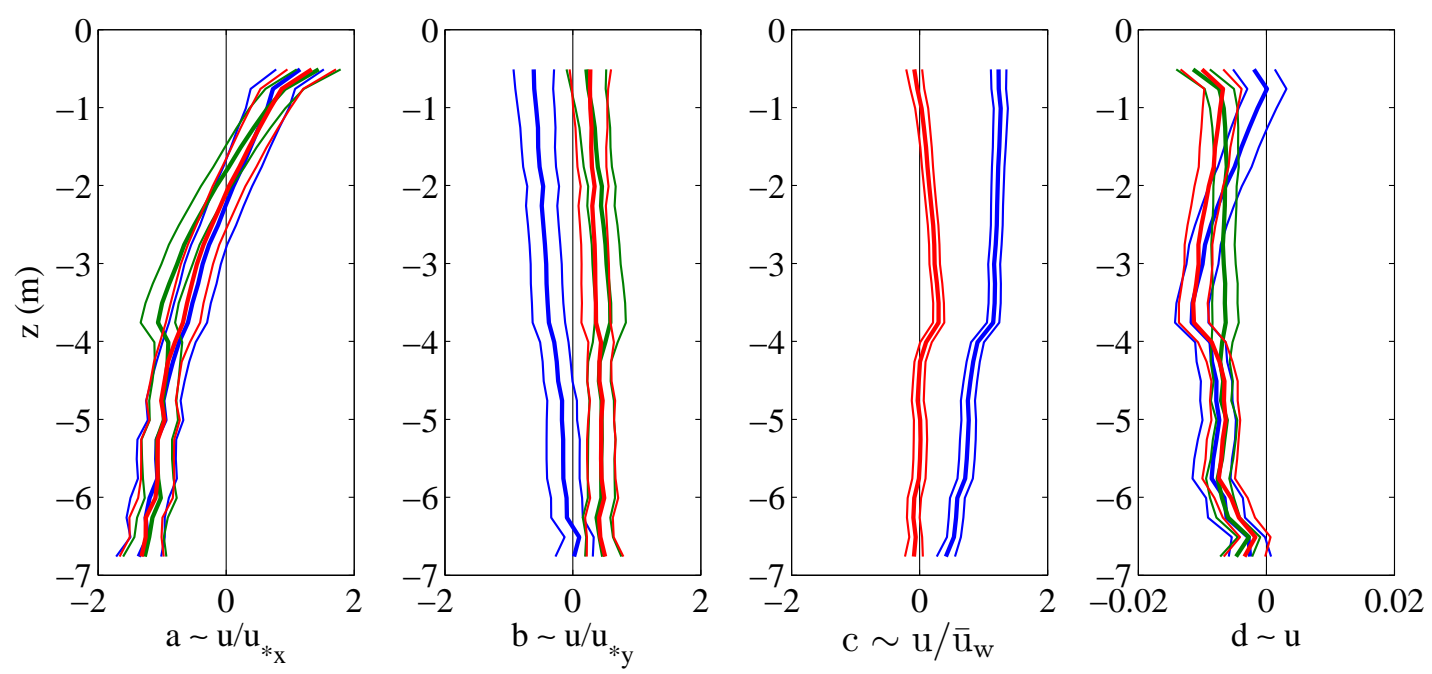

(a) mixed conditions
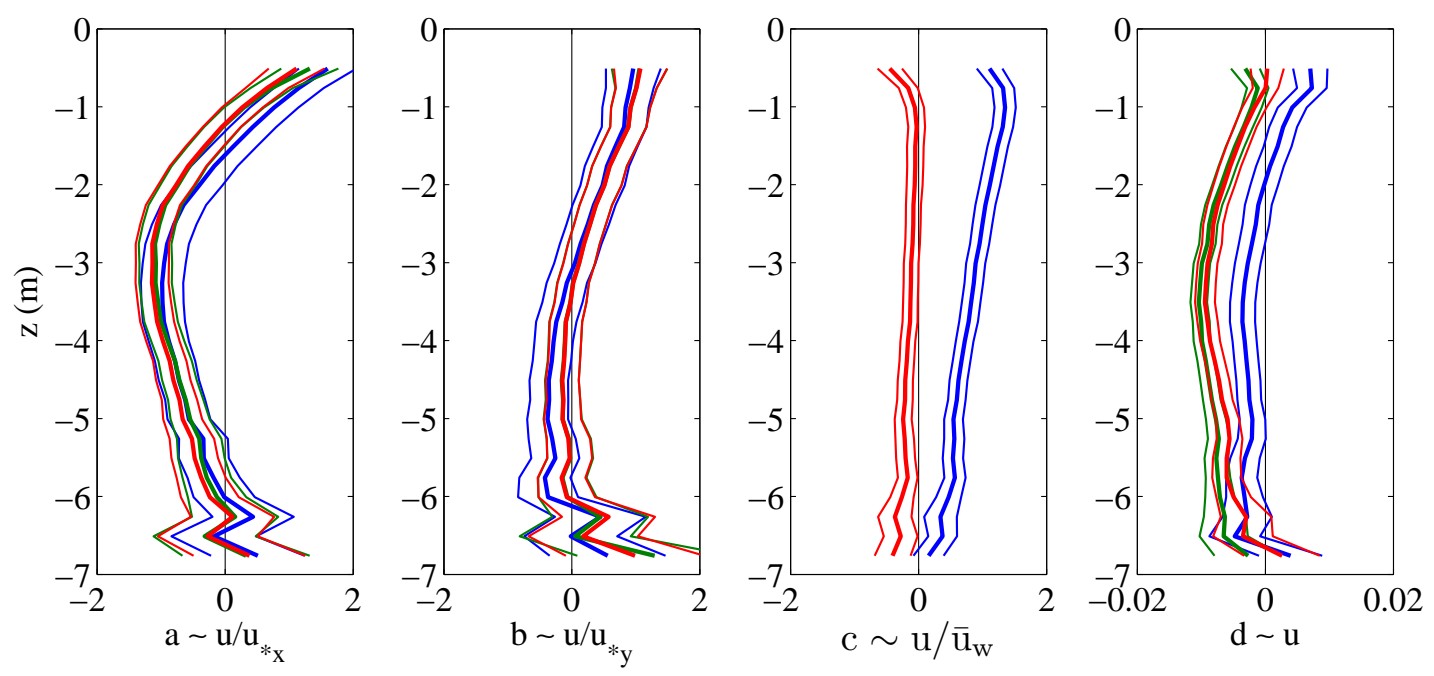

(b) stratified conditions

Figure 4-21: 7-m site: regression coefficients between ADCP velocity and, left to right, $u_{*}^{x}, u_{*}^{y}, u_{w}$, constant $=1$. Regressions using full velocity data with equation $4.2 \mathrm{~d}$ (blue), and velocity with predicted wave undertow profile subtracted using equations $4.2 \mathrm{~d}$ (red) and $4.2 \mathrm{a}$ (green) 

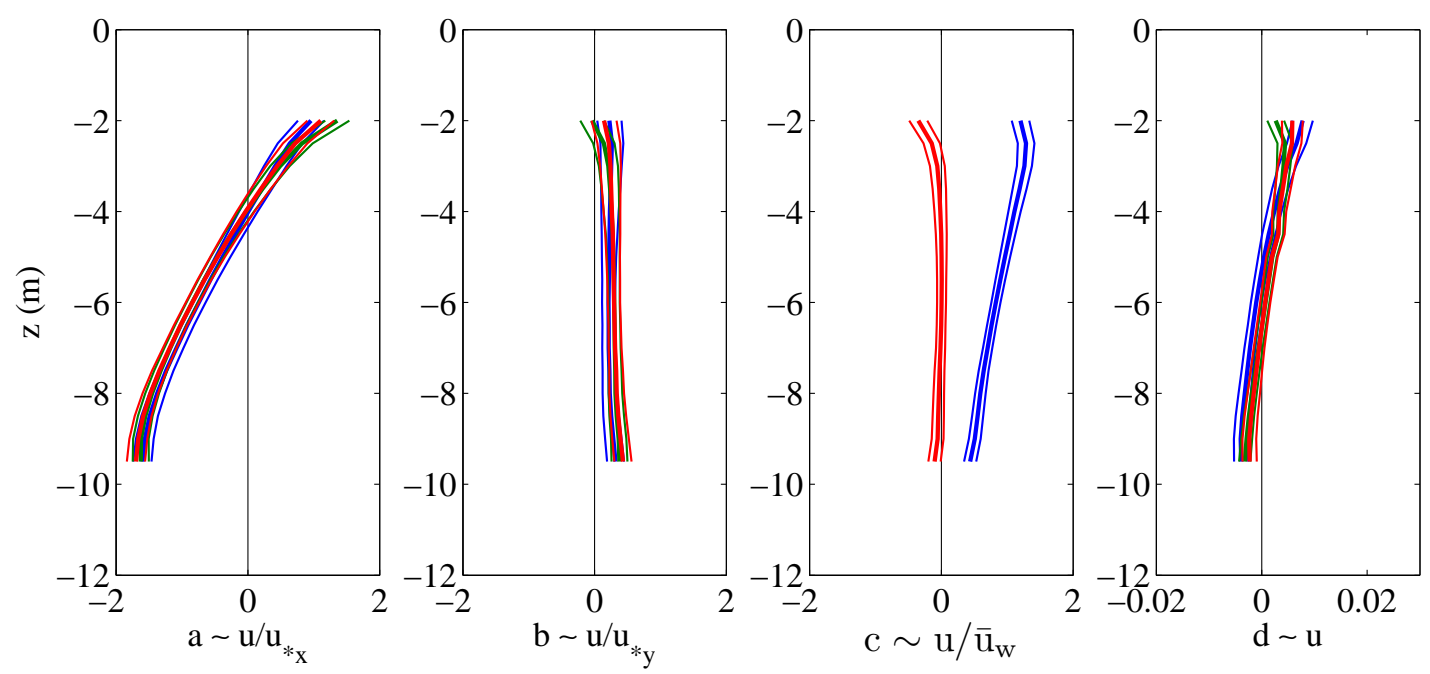

(a) mixed conditions
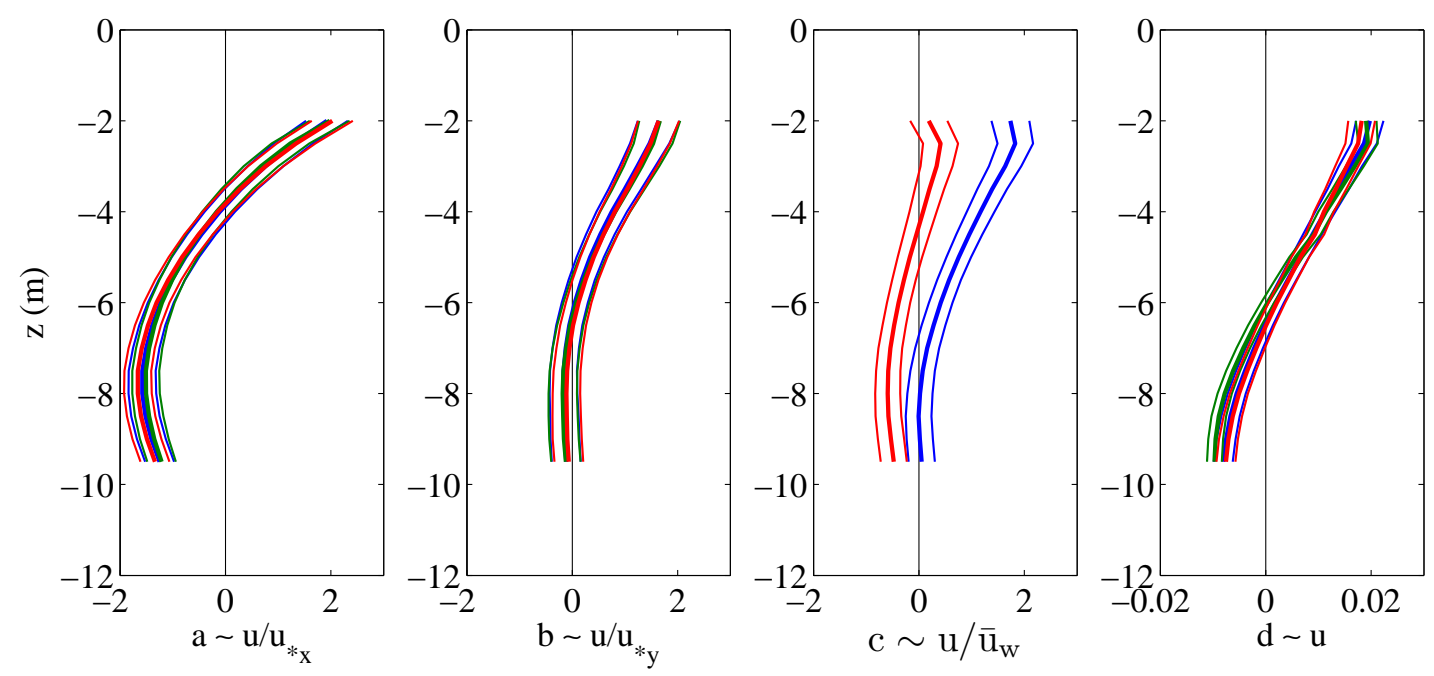

(b) stratified conditions

Figure 4-22: 12-m site: regression coefficients between ADCP velocity and, left to right, $u_{*}^{x}, u_{*}^{y}, u_{w}$, constant $=1$. Regressions using full velocity data with equation $4.2 \mathrm{~d}$ (blue), and velocity with predicted wave undertow profile subtracted using equations $4.2 \mathrm{~d}$ (red) and $4.2 \mathrm{a}$ (green) 

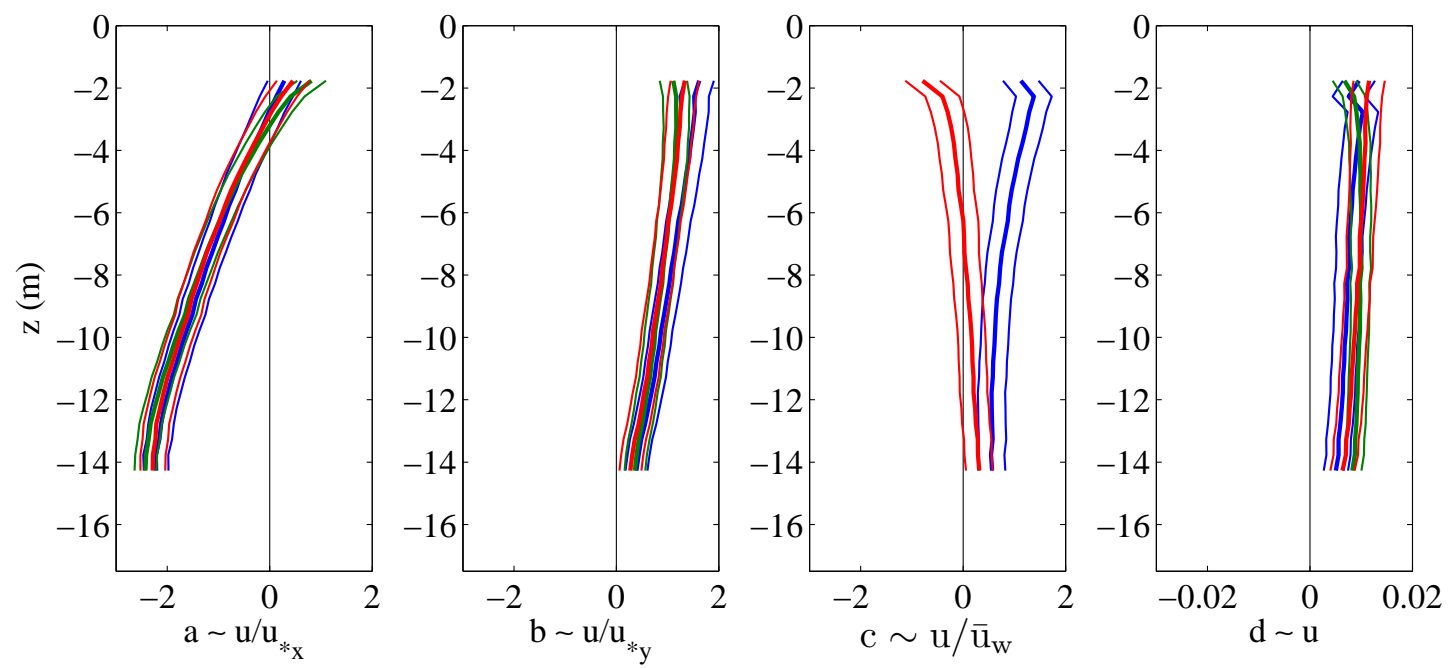

(a) mixed conditions
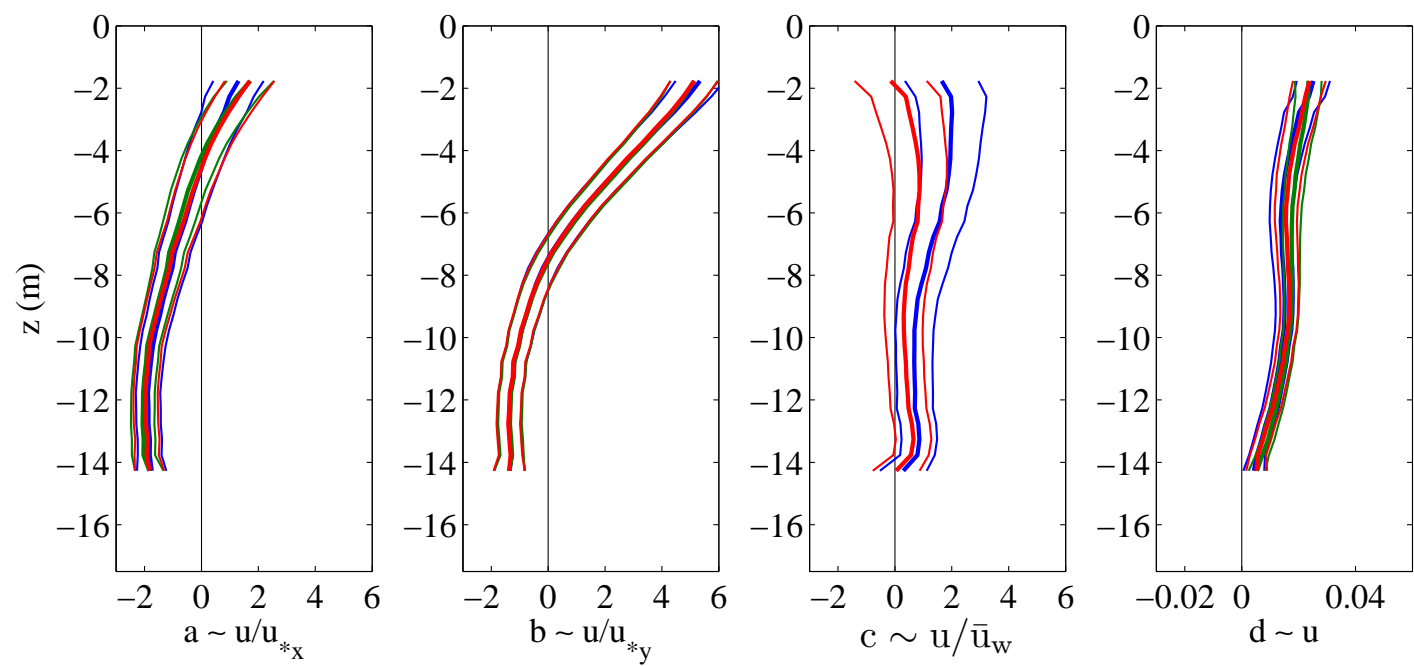

(b) stratified conditions

Figure 4-23: 17-m site: regression coefficients between ADCP velocity and, left to right, $u_{*}^{x}, u_{*}^{y}, u_{w}$, constant $=1$. Regressions using full velocity data with equation $4.2 \mathrm{~d}$ (blue), and velocity with predicted wave undertow profile subtracted using equations $4.2 \mathrm{~d}$ (red) and $4.2 \mathrm{a}$ (green) 

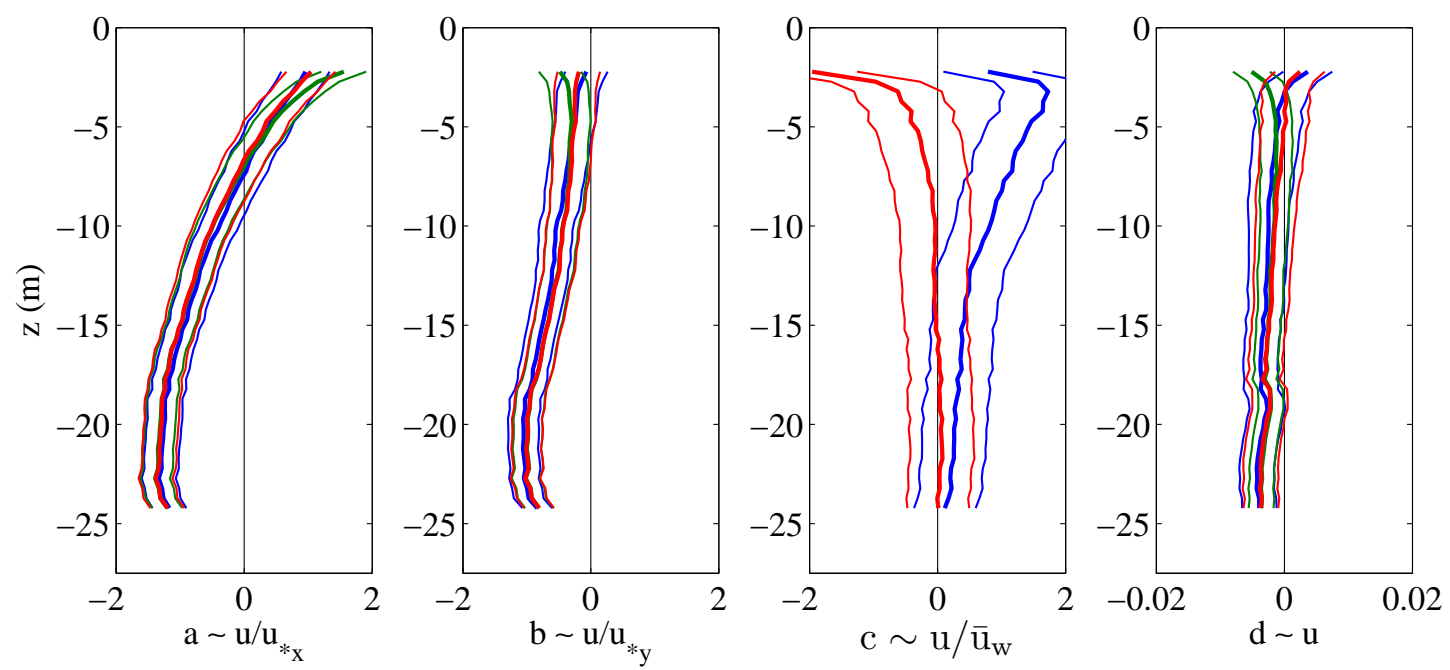

(a) mixed conditions
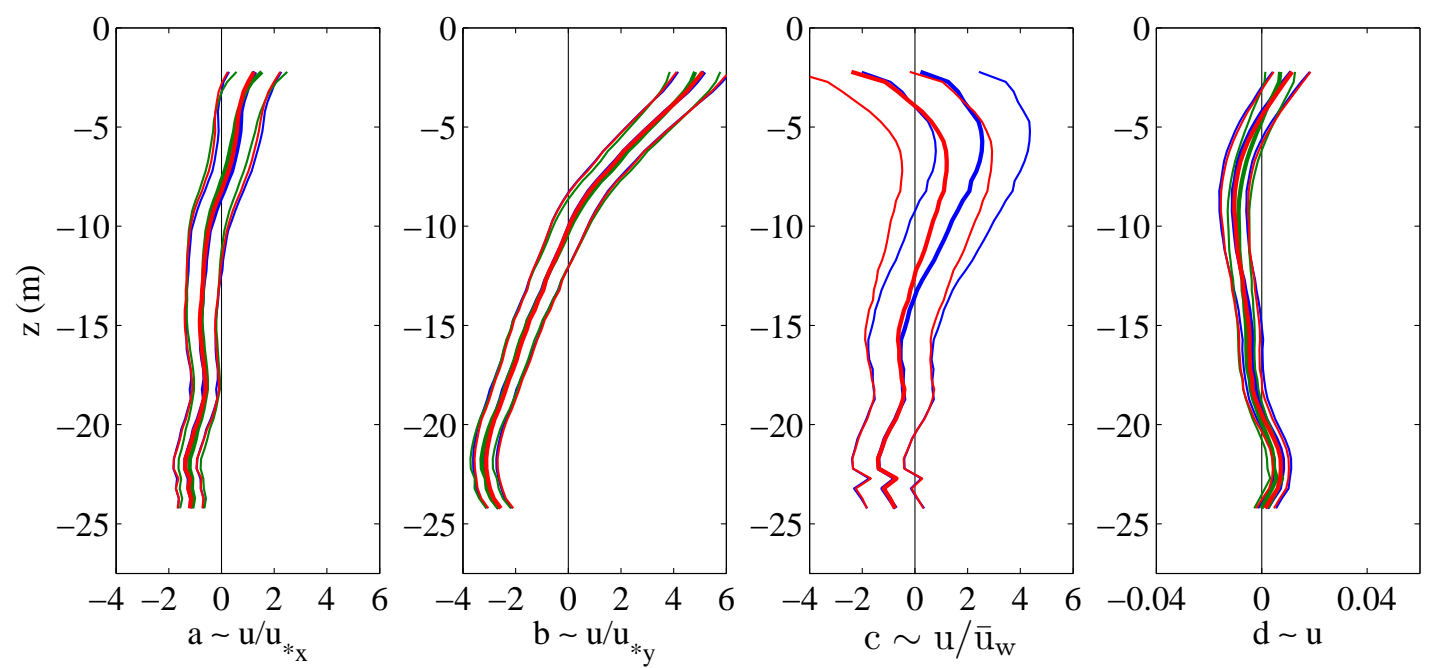

(b) stratified conditions

Figure 4-24: 27-m site: regression coefficients between ADCP velocity and, left to right, $u_{*}^{x}, u_{*}^{y}, u_{w}$, constant $=1$. Regressions using full velocity data with equation $4.2 \mathrm{~d}$ (blue), and velocity with predicted wave undertow profile subtracted using equations $4.2 \mathrm{~d}$ (red) and $4.2 \mathrm{a}$ (green) 


\section{Chapter 5}

\section{Inner shelf response to cross-shelf wind stress in an idealized numerical model}

\section{$5.1 \quad$ Introduction}

Chapter 4 used field measurements from the inner shelf south of Martha's Vineyard, MA to describe the effect of stratification on wind-driven, cross-shelf circulation. Stratification increases the vertical shear of the circulation and the transport driven by cross-shelf wind stresses. This effect of stratification is evident in the response to offshore winds but not to onshore winds (Figure 4-16). Our observations of circulation throughout a stratified water column and the asymmetric response to onshore and offshore winds are contrary to results from a previous numerical modeling study (Tilburg, 2003) that found circulation by cross-shelf wind stress was symmetric and confined to the surface mixed layer.

To resolve the discrepancy between our observations and previous modeling work, here we use a numerical model of an idealized, 2D, cross-shelf transect to describe the effects of stratification, wind stress magnitude, surface heat flux, cross-shelf density gradient, and wind direction on the inner shelf response to the cross-shelf component 
of the wind stress. These factors are all present in the field but difficult to isolate in the observations. A surface heat flux could add stratification by heating the near surface water, making it less dense than the water below, if this process can occur faster than wind mixing can distribute heat through the water column. A cross-shelf density gradient could add stratification by slumping during thermal wind spin up, or by advection by a vertically sheared wind-driven cross-shelf circulation. Our field observations of an asymmetric response to onshore and offshore wind stresses, the predicted asymmetry between up- and downwelling circulations in a stratified 2D model (Austin and Lentz, 2002), and the nonlinear nature of turbulent processes all motivated the investigation of the effect of an along-shelf wind component on the stratified response to cross-shelf wind forcing.

In this chapter, section 5.2 describes the ROMS model setup; section 5.3 presents experiments varying wind stress magnitude and initial stratification using the same set of forcing conditions as Tilburg (2003), while focusing on the circulation on the inner rather than midshelf; section 5.4 isolates the effect of the cross-shelf density gradient; section 5.5 adds a surface heat flux; section 5.6 uses combined cross- and along-shelf wind stresses; section 5.7 concludes with a summary of the results.

\subsection{Numerical Model}

The Regional Ocean Modeling System (ROMS) is used to simulate inner shelf dynamics for all numerical experiments described in this chapter. ROMS has been used extensively in previous idealized and realistic numerical experiments. Extensive information about the model is available on the ROMS ( http://www.myroms.org/ ) and WikiROMS ( https://www.myroms.org/wiki/ ) websites. This section describes the

numerical model, physical parameters selected for the "base case", and the conditions varied over subsequent suites of model runs. 


\subsubsection{ROMS model}

ROMS is a free-surface, terrain following, primitive equations ocean model with a curvilinear orthogonal horizontal grid and a stretched terrain-following vertical grid based on Shchepetkin and McWilliams (2005). The equations of motion for conservation of momentum and scalar tracers are given by

$$
\begin{aligned}
\frac{\partial u}{\partial t}+\vec{u} \cdot \nabla u-f v & =-\frac{1}{\rho_{o}} \frac{\partial P}{\partial x}-\frac{\partial}{\partial z}\left(\overline{u^{\prime} w^{\prime}}-\nu \frac{\partial u}{\partial z}\right) \\
\frac{\partial v}{\partial t}+\vec{u} \cdot \nabla v+f u & =-\frac{1}{\rho_{o}} \frac{\partial P}{\partial y}-\frac{\partial}{\partial z}\left(\overline{v^{\prime} w^{\prime}}-\nu \frac{\partial v}{\partial z}\right) \\
\frac{\partial C}{\partial t}+\vec{v} \cdot \nabla C & =-\frac{\partial}{\partial z}\left(\overline{C^{\prime} w^{\prime}}-\nu_{\theta} \frac{\partial C}{\partial z}\right) \\
\frac{1}{\rho_{o}} \frac{\partial P}{\partial z} & =-\frac{\rho g}{\rho_{o}} \\
\frac{\partial u}{\partial x}+\frac{\partial v}{\partial y}+\frac{\partial w}{\partial z} & =0
\end{aligned}
$$

where $\nu$ and $\nu_{\theta}$ are molecular viscosity and diffusivity. The Coriolis parameter is a constant $f=f_{0}=10^{-4}\left(\mathrm{~s}^{-1}\right)$ for all runs unless otherwise noted. Density varies with a linear equation of state, $\rho=\rho(T, S, P)$. Time steps are 10 seconds for the barotropic part of the equations and 50 or 100 seconds for the baroclinic terms. All experiments were run for 5 model days with variables saved hourly.

\section{Grid}

The model domain is a periodic channel with sloping boundaries to represent two along-shelf uniform coastal regions that span from just outside the surfzone to the flatter mid continental shelf. The cross-shelf section has a shallow $(2 \mathrm{~m})$ coastal wall at each boundary. Depth, $h$, increases away from each wall at a constant slope until the bathymetry reaches its maximum depth (65 $\mathrm{m}$ for the base case) at $63 \mathrm{~km}$ from the wall. The two sloping boundaries are separated by a $45 \mathrm{~km}$ wide uniform depth region in the center of the domain for a total domain width of $171 \mathrm{~km}$. The stretched vertical grid has 32 levels with enhanced resolution near the surface and 
bottom (ROMS options: Vtransform $=2$, Vstretching $=4, \theta_{S}=\theta_{B}=3$, Tcline $=25$ $\mathrm{m})$. At the coastal wall, the smallest vertical bin is $0.059 \mathrm{~m}$ and the largest is 0.065 $\mathrm{m}$. For all runs with base case bottom slope $\left(10^{-3}\right)$, as described in section 5.2.2, at the center of the domain the smallest vertical bin is $0.78 \mathrm{~m}$ and the largest is $2.81 \mathrm{~m}$. The vertical grid spacing varies slightly with the free surface elevation. The periodic along-shelf direction is $8 \mathrm{~km}$ long and the horizontal resolution is $1 \mathrm{~km} \times 1 \mathrm{~km}$ for the whole domain.

The model is run with the cross-shelf coordinate positive and increasing to the right as shown on the left side of Figure 5-1. However, with the spatially uniform wind stresses that are applied to this model domain, the opposite edge of the channel experiences opposite wind stresses relative to the coast. All data analysis presented in this chapter will use an "East Coast", right-handed, coordinate system. The cross-shelf coordinate, $x$, is zero at the coast and increases offshore. The along-shelf coordinate, $y$, is positive in the direction of an upwelling-favorable wind stress. We use the single model domain, shown on the left of Figure 5-1, but present results as the two coasts modeled with opposite wind forcing, shown on the right of the same figure.
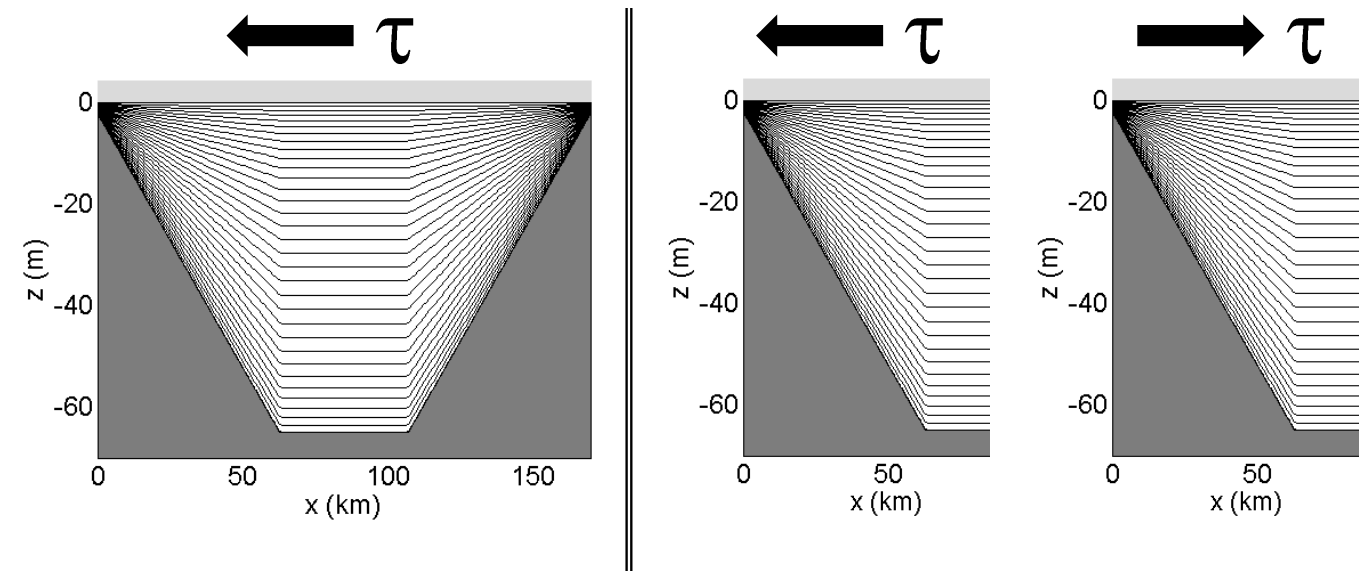

Figure 5-1: Left: model domain cross-section with wind forcing. Right: model output, as interpreted in data analysis 


\section{Mixing}

In ROMS, the system of governing equation is closed by parameterizing Reynolds stresses and turbulent fluxes as

$$
\overline{u^{\prime} w^{\prime}}=-K_{m} \frac{\partial u}{\partial z} ; \quad \overline{v^{\prime} w^{\prime}}=-K_{m} \frac{\partial v}{\partial z} ; \quad \overline{C^{\prime} w^{\prime}}=-K_{C} \frac{\partial C}{\partial z}
$$

with boundary conditions at the surface $(z=\zeta(x, y, t))$

$$
K_{m} \frac{\partial u}{\partial z}=\frac{1}{\rho} \tau^{s x}(x, y, t) ; \quad K_{m} \frac{\partial v}{\partial z}=\frac{1}{\rho} \tau^{s y}(x, y, t) ; \quad K_{C} \frac{\partial C}{\partial z}=\frac{Q_{C}}{\rho_{o} c_{P}} ; \quad w=\frac{\partial \zeta}{\partial t}
$$

and bottom $(z=-h(x, y))$

$$
K_{m} \frac{\partial u}{\partial z}=\frac{1}{\rho} \tau^{b x}(x, y, t) ; \quad K_{m} \frac{\partial v}{\partial z}=\frac{1}{\rho} \tau^{b y}(x, y, t) ; \quad K_{C} \frac{\partial C}{\partial z}=0 ; \quad-w+\vec{v} \cdot \nabla h=0
$$

where $\tau^{s x}, \tau^{s y}$ and $\tau^{b x}, \tau^{b y}$ are the cross- and along-shelf components of the surface and bottom stresses. Eddy viscosity and diffusivity are estimated by the Mellor-Yamada 2.5 (1982) turbulence closure scheme with the Kantha-Clayson (1994) stability function. The background value of vertical viscosity, $K_{m}$, is $10^{-5} \mathrm{~m}^{2} / \mathrm{s}$, and the background value of vertical diffusivity for temperature, $K_{C}$, is $10^{-6} \mathrm{~m}^{2} / \mathrm{s}$. A logarithmic bottom drag coefficient is applied at the center height of each bottom grid cell, which varies in height with our stretched grid. The resulting stress is quadratic with local velocity scaled by the log of the distance from the apparent bottom roughness, $z_{0}=$ $1 \mathrm{~cm}$

$$
\frac{\vec{\tau}^{b}}{\rho}=\vec{u}|\vec{u}| \frac{\kappa^{2}}{\left(\ln \frac{z}{z_{0}}\right)^{2}}
$$




\subsubsection{Base Case}

We first describe the parameters in a base case model setup. All other runs start with these parameters, initial conditions, and wind forcing unless otherwise specified. The base case run has a bottom slope of $\alpha=10^{-3}$, for a maximum depth of $65 \mathrm{~m}$. The model is initialized with a constant vertical temperature gradient of $0.25^{\circ} \mathrm{C} / \mathrm{m}$, which creates horizontal isopycnals and an initial stratification of $N^{2}=4.25 \times 10^{-4}$ $\mathrm{s}^{-2}$ (Figure 5-2). In the base case, wind stress is in the cross-shelf direction only, with no along-shelf component. The wind stress magnitude begins at zero and increases over the first two days as $\tau_{s}(t)=-0.1 \sin (\pi t / 4)$, where $t$ is time in days. The wind stress is steady over the final three days of model time with $\tau^{x}=-0.1 \mathrm{~Pa}, \tau^{y}=0$. The negative sign of the cross-shelf wind means the stress is onshore on the left side of our domain as shown in Figure 5-2, and offshore on the right.

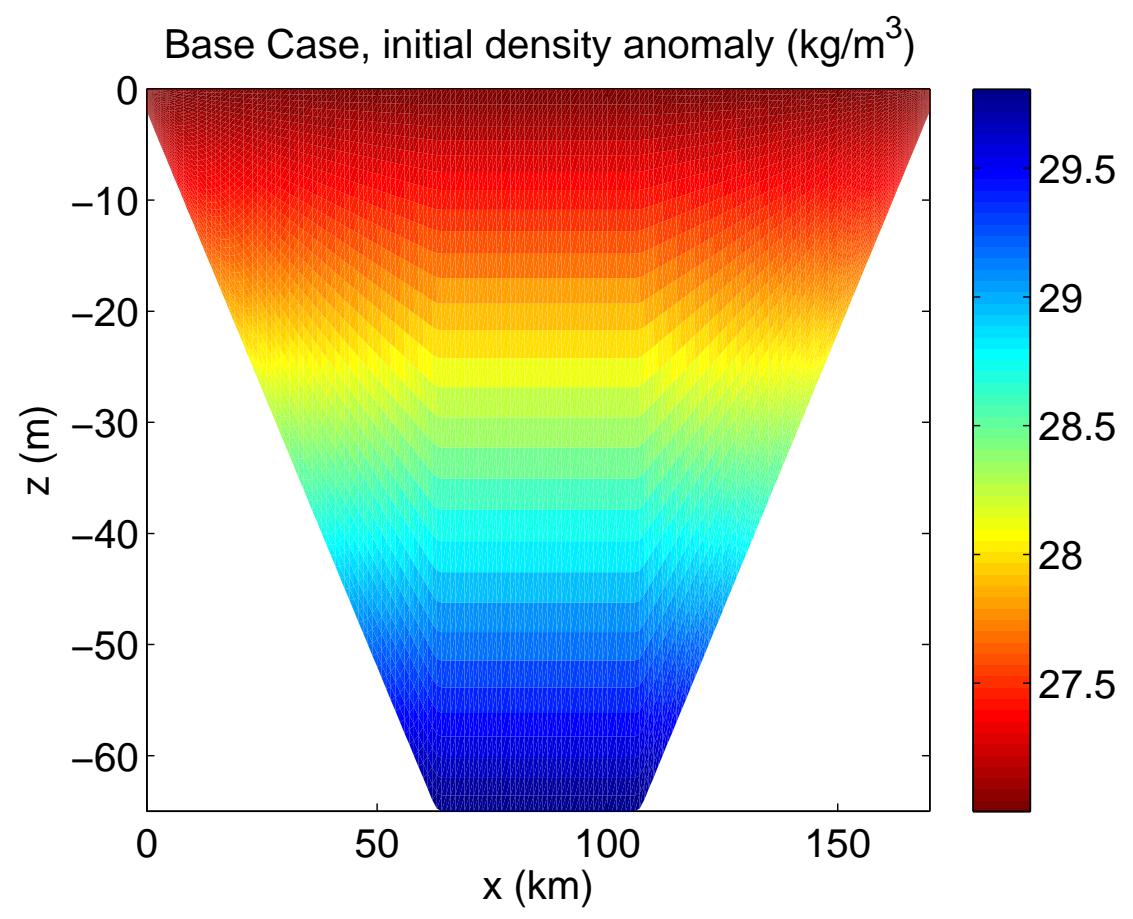

Figure 5-2: Cross-shelf section of initial density anomaly, $\rho-1000\left(\mathrm{~kg} / \mathrm{m}^{3}\right)$, for base case 


\subsubsection{Model runs}

Tables 5.1 and 5.2 summarize all model implementations performed for the experiments in this chapter. Runs used for model testing but not specifically addressed in this chapter are also included here for completeness. Table 5.1 includes runs for all experiments that use purely cross-shelf wind stresses, which are described in sections 5.3, 5.4, and 5.5. Table 5.2 includes runs for the experiment using combined crossand along-shelf wind stresses, which is described in section 5.6. In most analyses in this chapter, the base case run (top of table 5.1) is included and discussed as part of the suite of model runs under investigation. In figures in this chapter, the base case is indicated $(\circ)$, varying wind stress magnitude $(\triangle)$, initial stratification $(\diamond)$, water depth $(*)$ or $(\cdot)$, bottom slope $(\square)$, initial cross-shelf density gradient $(*)$, and surface heat flux $(+)$.

\subsection{Scaling inner shelf circulation by basic forcing mechanisms: varying $N_{0}^{2}$ and $\tau^{x}$}

Here we explore circulation and transport fraction on the inner shelf, much like Tilburg (2003) did for the midshelf, with added focus on the differences between onshore and offshore wind stress responses. First, we use a scaling analysis to determine the expected response of the circulation. Assuming uniform density and a steady, linear balance, the cross-shelf momentum equation is

$$
f v=g \frac{\partial \eta}{\partial x}-\frac{1}{\rho_{o}} \frac{\partial \tau^{x}}{\partial z}
$$

The surface wind stress is the only forcing for this system and so provides the velocity scale, the shear velocity, $u_{*}^{2} \equiv \tau^{s} / \rho$. The observations from Chapter 4 have demonstrated that the proportionality coefficient between the driven velocity, $u$, and shear velocity, $u_{*}$ is order one since almost all regression slope coefficients between $u$ and $u_{*}^{x}$ or $u_{*}^{y}$ calculated in Chapter 4 are between zero and five. 
Table 5.1: Model runs with cross-shelf wind stress only

\begin{tabular}{|c|c|c|c|c|c|c|c|c|c|}
\hline \multirow[t]{2}{*}{ Experiment } & \multirow[t]{2}{*}{$\begin{array}{l}\left|\tau^{s}\right| \\
(\mathrm{Pa})\end{array}$} & \multirow[t]{2}{*}{$\begin{array}{l}\text { Initial } N^{2} \\
\quad\left(\mathrm{~s}^{-2}\right)\end{array}$} & \multicolumn{2}{|c|}{$\begin{array}{l}\text { Wind dir. }{ }^{\mathrm{a}} \\
\left({ }^{\circ}\right)\end{array}$} & \multirow[t]{2}{*}{$\begin{array}{c}\alpha \\
10^{-3}\end{array}$} & \multirow[t]{2}{*}{$\begin{array}{c}Q \\
\left(\frac{\mathrm{W}}{\mathrm{m}^{2}}\right)\end{array}$} & \multicolumn{2}{|c|}{$\begin{array}{c}\partial \rho / \partial x \\
10^{-5}\left(\mathrm{~kg} / \mathrm{m}^{4}\right)\end{array}$} & \multirow[t]{2}{*}{$\begin{array}{c}f \\
10^{-4}\left(\mathrm{~s}^{-1}\right)\end{array}$} \\
\hline & & & Near $^{b}$ & Far $^{\mathrm{c}}$ & & & Near & Far & \\
\hline Base Case & 0.1 & $4.25 \times 10^{-4}$ & 180 & 0 & 1.0 & 0 & 0 & 0 & 1.0 \\
\hline $\begin{array}{l}\text { Unstratified } \\
\text { Vary } \tau^{x}\end{array}$ & $\begin{array}{l}0.01 \\
0.013 \\
0.023 \\
0.036 \\
0.05 \\
0.1 \\
0.2 \\
0.36\end{array}$ & 0 & 180 & 0 & 1.0 & 0 & 0 & 0 & 1.0 \\
\hline $\operatorname{Vary} \tau^{x}$ & $\begin{array}{l}0.01 \\
0.05 \\
0.2 \\
0.36\end{array}$ & $4.25 \times 10^{-4}$ & 180 & 0 & 1.0 & 0 & 0 & 0 & 1.0 \\
\hline Vary $N^{2}$ & 0.1 & $\begin{array}{l}4.25 \times 10^{-6} \\
2.13 \times 10^{-5} \\
8.50 \times 10^{-5} \\
8.50 \times 10^{-4} \\
1.06 \times 10^{-3}\end{array}$ & 180 & 0 & 1.0 & 0 & 0 & 0 & 1.0 \\
\hline Vary $\alpha$ & 0.1 & $4.25 \times 10^{-4}$ & 180 & 0 & $\begin{array}{l}0.5 \\
2.0\end{array}$ & 0 & 0 & 0 & 1.0 \\
\hline \multirow[t]{2}{*}{$\operatorname{Vary} \partial \rho / \partial x$} & 0.1 & $1.70 \times 10^{-4}$ & 180 & 0 & 1.0 & 0 & $\begin{array}{r}1.75 \\
3.49 \\
8.73 \\
-1.75 \\
-3.49 \\
-8.73\end{array}$ & $\begin{array}{r}-1.75 \\
-3.49 \\
-8.73 \\
1.75 \\
3.49 \\
8.73\end{array}$ & 1.0 \\
\hline & 0 & $1.70 \times 10^{-4}$ & 180 & 0 & 1.0 & 0 & $\begin{array}{l}1.75 \\
3.49 \\
8.73 \\
\end{array}$ & $\begin{array}{l}-1.75 \\
-3.49 \\
-8.73 \\
\end{array}$ & 1.0 \\
\hline \multirow[t]{2}{*}{ Vary $Q$} & 0.1 & $4.25 \times 10^{-4}$ & 180 & 0 & 1.0 & $\begin{array}{r}-10 \\
10 \\
50 \\
100 \\
150 \\
200\end{array}$ & 0 & 0 & 1.0 \\
\hline & 0.05 & $4.25 \times 10^{-4}$ & 180 & 0 & 1.0 & $\begin{array}{r}50 \\
150\end{array}$ & 0 & 0 & 1.0 \\
\hline Vary $f$ & 0.1 & $4.25 \times 10^{-4}$ & 90 & 270 & 1.0 & 0 & 0 & 0 & $\begin{array}{l}0.5 \\
1.3\end{array}$ \\
\hline
\end{tabular}

${ }^{a}$ Wind direction is direction of surface wind stress. $0^{\circ}$ is offshore, $90^{\circ}$ is upwelling.

${ }^{\mathrm{b}}$ Variable used in model coordinates, and for data analysis on near coast of model domain.

${ }^{\mathrm{c}}$ Variable experienced by the far coast of model domain in local coordinate. See Fig. 5-1. 
Table 5.2: Model runs for combined cross- and along-shelf wind forcing

\begin{tabular}{|c|c|c|c|c|c|c|c|c|c|}
\hline \multirow[t]{2}{*}{ Experiment } & \multirow[t]{2}{*}{$\begin{array}{l}\left|\tau^{s}\right| \\
(\mathrm{Pa})\end{array}$} & \multirow[t]{2}{*}{$\begin{array}{c}\text { Initial } N^{2} \\
\left(\mathrm{~s}^{-2}\right)\end{array}$} & \multicolumn{2}{|c|}{$\begin{array}{l}\text { Wind dir. } \\
\left({ }^{\circ}\right)\end{array}$} & \multirow[t]{2}{*}{$\begin{array}{c}\alpha \\
10^{-3}\end{array}$} & \multirow[t]{2}{*}{$\begin{array}{c}Q \\
\left(\frac{\mathrm{W}}{\mathrm{m}^{2}}\right)\end{array}$} & \multicolumn{2}{|c|}{$\begin{array}{c}\partial \rho / \partial x \\
\left(\mathrm{~kg} / \mathrm{m}^{4}\right)\end{array}$} & \multirow[t]{2}{*}{$\begin{array}{c}f \\
10^{-4}\left(\mathrm{~s}^{-1}\right)\end{array}$} \\
\hline & & & Near $^{b}$ & Far $^{\mathrm{C}}$ & & & Near & Far & \\
\hline $\operatorname{Vary} \tau^{y}$ & $\begin{array}{l}0.01 \\
0.05 \\
0.2 \\
0.36 \\
\end{array}$ & $4.25 \times 10^{-4}$ & 90 & 270 & 1.0 & 0 & 0 & 0 & 1.0 \\
\hline $\begin{array}{l}\text { Vary } \theta \\
\text { (wind dir.) }\end{array}$ & 0.1 & $4.25 \times 10^{-4}$ & $\begin{array}{r}165 \\
150 \\
135 \\
120 \\
105 \\
90 \\
75 \\
60 \\
45 \\
30 \\
15\end{array}$ & $\begin{array}{l}345 \\
330 \\
315 \\
300 \\
285 \\
270 \\
255 \\
240 \\
225 \\
210 \\
195\end{array}$ & 1.0 & 0 & 0 & 0 & 1.0 \\
\hline $\begin{array}{l}\tau^{x} \text { compo- } \\
\text { nent } \\
\text { of runs } \\
\text { varying } \theta\end{array}$ & $\begin{array}{l}0.1 \sin \left(15^{\circ}\right) \\
0.1 \sin \left(30^{\circ}\right) \\
0.1 \sin \left(45^{\circ}\right) \\
0.1 \sin \left(60^{\circ}\right) \\
0.1 \sin \left(75^{\circ}\right)\end{array}$ & $4.25 \times 10^{-4}$ & 180 & 0 & 1.0 & 0 & 0 & 0 & 1.0 \\
\hline $\begin{array}{l}\tau^{y} \quad \text { compo- } \\
\text { nent } \\
\text { of runs } \\
\text { varying } \theta\end{array}$ & $\begin{array}{l}0.1 \cos \left(15^{\circ}\right) \\
0.1 \cos \left(30^{\circ}\right) \\
0.1 \cos \left(45^{\circ}\right) \\
0.1 \cos \left(60^{\circ}\right) \\
0.1 \cos \left(75^{\circ}\right)\end{array}$ & $4.25 \times 10^{-4}$ & 90 & 270 & 1.0 & 0 & 0 & 0 & 1.0 \\
\hline
\end{tabular}

${ }^{a}$ Wind direction is direction of surface wind stress. $0^{\circ}$ is offshore, $90^{\circ}$ is upwelling.

b Variable used in model coordinates, and for data analysis on near coast of model domain.

${ }^{\mathrm{c}}$ Variable experienced by the far coast of model domain in local coordinates. See Fig. 5-1. 
In the deep water limit, away from coastal boundaries, no pressure gradient can develop and the momentum equation reduces to

$$
f v=-\frac{1}{\rho_{o}} \frac{\partial \tau}{\partial z}
$$

Taking the velocities to scale like $u_{*}$ and choosing $z^{+}$as the unknown vertical length scale, then the term of equation 5.6 scale as

$$
f u_{*}=\frac{u_{*}^{2}}{z^{+}}
$$

and solving for the vertical length scale, we find

$$
z^{+}=\frac{u_{*}}{f} \sim \delta_{E k}
$$

where $\delta_{E k}=\kappa u_{*} / f$ is the deep water boundary layer thickness, which includes the empirically derived Von Kármán constant, $\kappa=0.41$. Boundary layer transport, $U$ scales like the velocity scale times the vertical length scale, $u_{*} z^{+}=u_{*}^{2} / f$. By integrating equation 5.6 from the surface to where the stress goes to zero, Ekman (1905) showed that for transport to the right of the wind stress, the proportionality constant is one and $U=u_{*}^{2} / f$. For downwind transport, which would be found by integrating the along-shelf momentum equation to the first zero-crossing the of crossshelf velocity profile, the proportionality constant depends on the vertical mixing.

In the shallow water limit, the water depth, $h$ is much less than the turbulent boundary layer thickness so the total water depth is the relevant vertical length scale. Rescaling equation 5.5 using $z^{+}=h$

$$
f u_{*}=g \frac{\partial \eta}{\partial x}-\frac{u_{*}^{2}}{h}
$$


and dividing by $u_{*}^{2} / h$

$$
\frac{f h}{u_{*}}=\frac{g h \frac{\partial \eta}{\partial x}}{u_{*}^{2}}-1
$$

Where $h / \delta_{E k}=f h / \kappa u_{*}<<1$, the Coriolis term is not large enough to balance the order one stress term so it can be ignored. The resulting momentum balance is

$$
0=g \frac{\partial \eta}{\partial x}-\frac{1}{\rho_{o}} \frac{\partial \tau}{\partial z}
$$

In this shallow water limit, transport scales as the velocity scale times the vertical length scale, which now becomes $U \sim u_{*} z^{+}=u_{*} h$.

Between the deep and shallow water limits Ekman (1905) described the solution as a superposition of the two limits. The vertical length scale will be some function of both $h$ and $\delta_{E k}$ and the transition from deep to shallow defined by the ratio of the two vertical scales $h / \delta_{E k}$. For typical inner shelf values of $f=10^{-4} \mathrm{~s}^{-1}, h=10 \mathrm{~m}$, and $u_{*}=0.01 \mathrm{~m} / \mathrm{s}, h / \delta_{E k}=0.25$ and, likewise, the Coriolis term on the left side of equation $5.9 \mathrm{~b}$ is order $10^{-1}$. We proceed with our analysis by taking the inner shelf region to behave as the shallow water limit predicts, while recognizing that rotation plays a small but perhaps non-negligible role in the dynamics.

\subsubsection{Unstratified cases}

In unstratified conditions, when cross-shelf wind stress, $\tau_{s}^{x} / \rho$, is balanced by a crossshelf pressure gradient, we expect $u / u_{*}$ to scale like $z / h$ and $U \sim u_{*} h$ for transport above the first zero crossing of the velocity profile. The nondimensionalized crossshelf velocity profiles are expected to collapse together when scaled by shear velocity and water depth. Figure 5-3(a) shows profiles from $10 \mathrm{~m}$ water depth, always within the inner shelf $\left(h<\delta=\kappa u_{*} / f\right)$, for a range of cross-shelf wind stresses from -0.01 to $-0.36 \mathrm{~Pa}$, yielding shear velocity values $u_{*}=0.31$ to $1.9 \mathrm{~cm} / \mathrm{s}$. The profile that is least sheared where all the profiles intersect is from the smallest wind stress and the 
most sheared profile is from the largest wind stress.

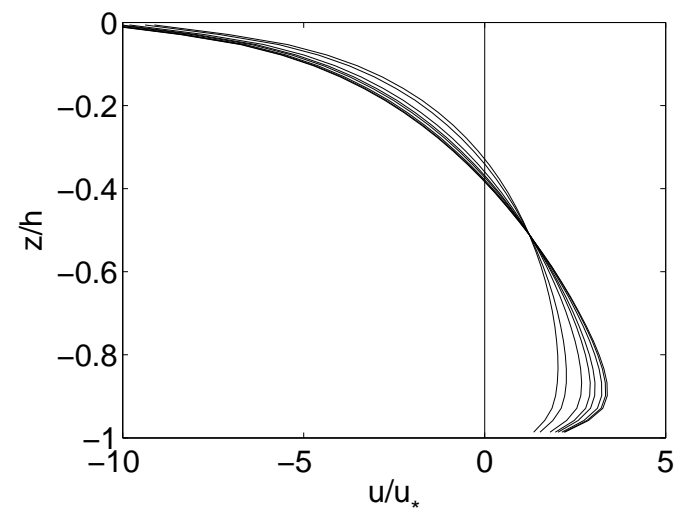

(a) Varying $|\tau|$

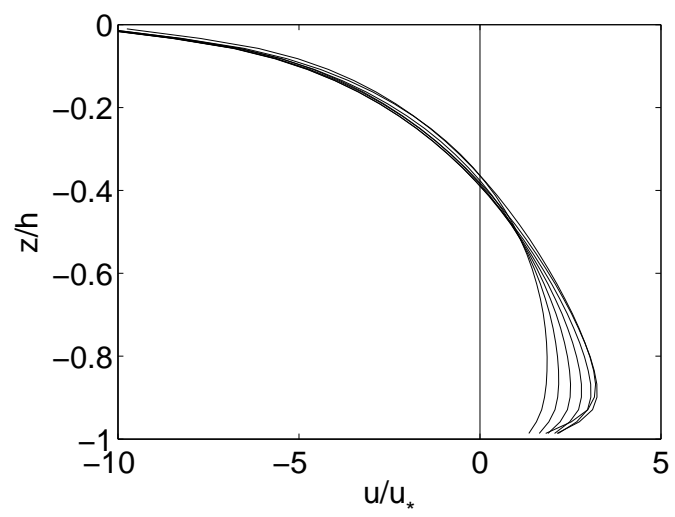

(b) Varying $h$

Figure 5-3: Nondimensional cross-shelf velocity profiles for unstratified runs. In 5$3(\mathrm{a})$, all profiles are from $10 \mathrm{~m}$ water depth and wind stress magnitude varies from -0.01 to $-0.36 \mathrm{~Pa}$. In 5-3(b), water depth at every $5 \mathrm{~m}$ from 5 to $35 \mathrm{~m}$, all with $\tau^{x}=$ $-0.1 \mathrm{~Pa}$.

In Figure 5-3(b), profiles from 5 to $35 \mathrm{~m}$ water depth from a single model run with $\tau=-0.1 \mathrm{~Pa}$ show a similar spread. The most sheared profile at the intersection point is from the shallowest site. A higher wind stress thickens the boundary layer, moving the transition to mid-shelf dynamics farther offshore.

In Figure 5-3, an onshore wind stress is used, but in unstratified experiments, the results for an offshore wind stress are nearly identical. In both cases, the spread in the profiles demonstrates a deviation from the $u / u_{*}, z / h$ scaling but can be explained by an increase in $h / \delta$ and gradual transition from the inner to mid-shelf following a transition from a pressure gradient - wind stress balance to a three term balance including the Coriolis acceleration.

The transition from inner to midshelf dynamics can be seen more clearly in the cross-shelf transport. Figure 5-4 shows the cross-shelf transport, $U$, calculated by integrating the cross-shelf velocity profiles from the surface down to the first zero crossing. Triangles indicate transport calculated at one location, $10 \mathrm{~m}$ water depth, for each of the unstratified runs. These runs had wind stress magnitudes of 0.01 , $0.013,0.023,0.036,0.05,0.1,0.2$ and $0.36 \mathrm{~Pa}$ so the $u_{*} h$ prediction varies because 
$u_{*}$ varies. The $10 \mathrm{~m}$ water depth is less than the deep water boundary layer scale $\delta_{E k}=\kappa u_{*} / f$ for all but the $0.01 \mathrm{~Pa}$ wind stress and transport increases linearly with $u_{*} h$ at a slope of approximately 1.3, indicated by the thin black line. The asterisks indicate transport calculated from two of the unstratified model runs with wind stress of 0.036 and $0.1 \mathrm{~Pa}$, at every $1 \mathrm{~m}$ increment in water depth from 4 to $65 \mathrm{~m}$, so for the asterisks, $h$ is varied in the $u_{*} h$ scaling. For increasing water depth, the transport also follows the $\sim 1.3 u_{*} h$ scale until the deep water transport value is approached, at which point $h$ continues to increase while $U$ remains constant. At midshelf, crossshelf transport reaches a constant value that is proportional to the wind stress and independent of the water depth. It should be noted that the down-wind transport in deep water is not independent of the vertical mixing as the cross-wind transport is. We find deep water transport values of $0.35-0.38 \times(\tau / \rho f)$ when $\kappa u_{*} / f$ is less than the water depth, $65 \mathrm{~m}$, at the center of our domain.

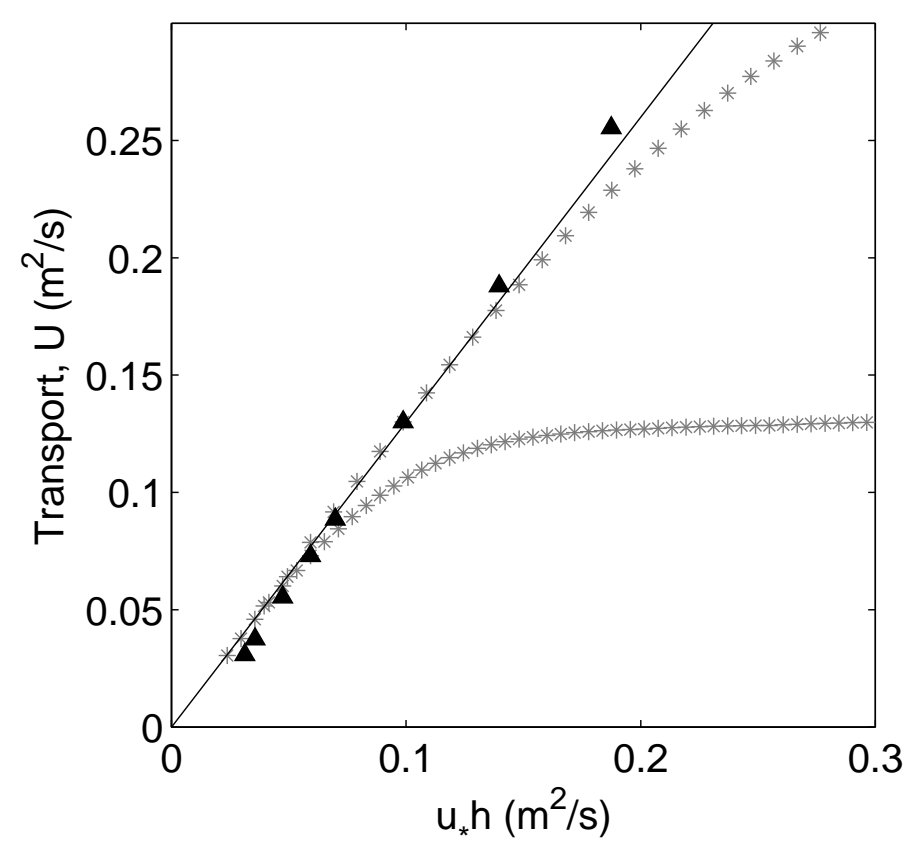

Figure 5-4: Transport, $U$, integrated from the surface to the first zero crossing of the cross-shelf velocity profile compared to the transport scale $u_{*} h$, surface shear velocity times water depth. Triangles indicate a depth of $10 \mathrm{~m}$ and $\tau^{x}$ varying from 0.01 to $0.36 \mathrm{~Pa}$. Asterisks indicate water depth varied from 4 to $65 \mathrm{~m}$ while $\tau^{x}$ was constant at 0.036 and $0.1 \mathrm{~Pa}$ for lower and higher lines. Solid line has slope of 1.3. 


\subsubsection{Initially stratified runs}

When wind blows across an initially stratified inner shelf, the stratification is mixed away, down to the bottom for all water depths less than the thickness of the surface boundary layer, creating a circulation that is much like the unstratified case. Figure 5-5 shows the inner shelf response to the base case onshore (top row) and offshore (bottom row) wind stresses. The cross-shelf velocities (5-5(b)) show both wind directions drive near-surface circulation in the direction of the wind stress with a return flow in the lower potion of the water column. Vertical mixing of the initial stratification creates a cross-shelf density gradient due to the bathymetry intersecting increasingly dense isopycnals with depth. Figure 5-5(a) shows the density field after five days of $0.1 \mathrm{~Pa}$ wind stress. For these, and all cases with initially horizontal isopycnals and stable stratification, the resulting cross-shelf density gradient is positive regardless of wind direction. The interaction of the wind-driven cross-shelf circulation with this cross-shelf density gradient is the primary source of the difference between the response to onshore and offshore wind stresses.

Offshore wind stress blows from the least dense region towards the denser, deeper region of the inner shelf and creates a very small local stratification as a result of buoyancy advection (bottom row, Figure 5-6). The stratification reduces mixing and in turn allows greater vertical shear and greater cross-shelf transport. The increased cross-shelf transport across the cross-shelf density gradient feeds back positively on the stratification. The transport does not run away, unbounded, with increasing stratification because the process is still limited by shear instabilities; the resulting $N^{2}=O\left(10^{-5}\right) \mathrm{s}^{-2}$ and the Richardson number is $\mathrm{O}\left(10^{-1}\right)$. Both values peak where there is near zero shear in the velocity at the height of the peak onshore return flow. Profiles of shear, stratification, Richardson number and eddy viscosity at $10 \mathrm{~m}$ water depth under an offshore wind stress on an initially stratified shelf can be see in the red lines of Figure 5-7. The largest Richardson number in this example is about 0.4 and limits the eddy viscosity to a constant $10^{-4} \mathrm{~m}^{2} / \mathrm{s}$ in the bottom few meters of the water column while eddy viscosity increases approximately linearly away from 

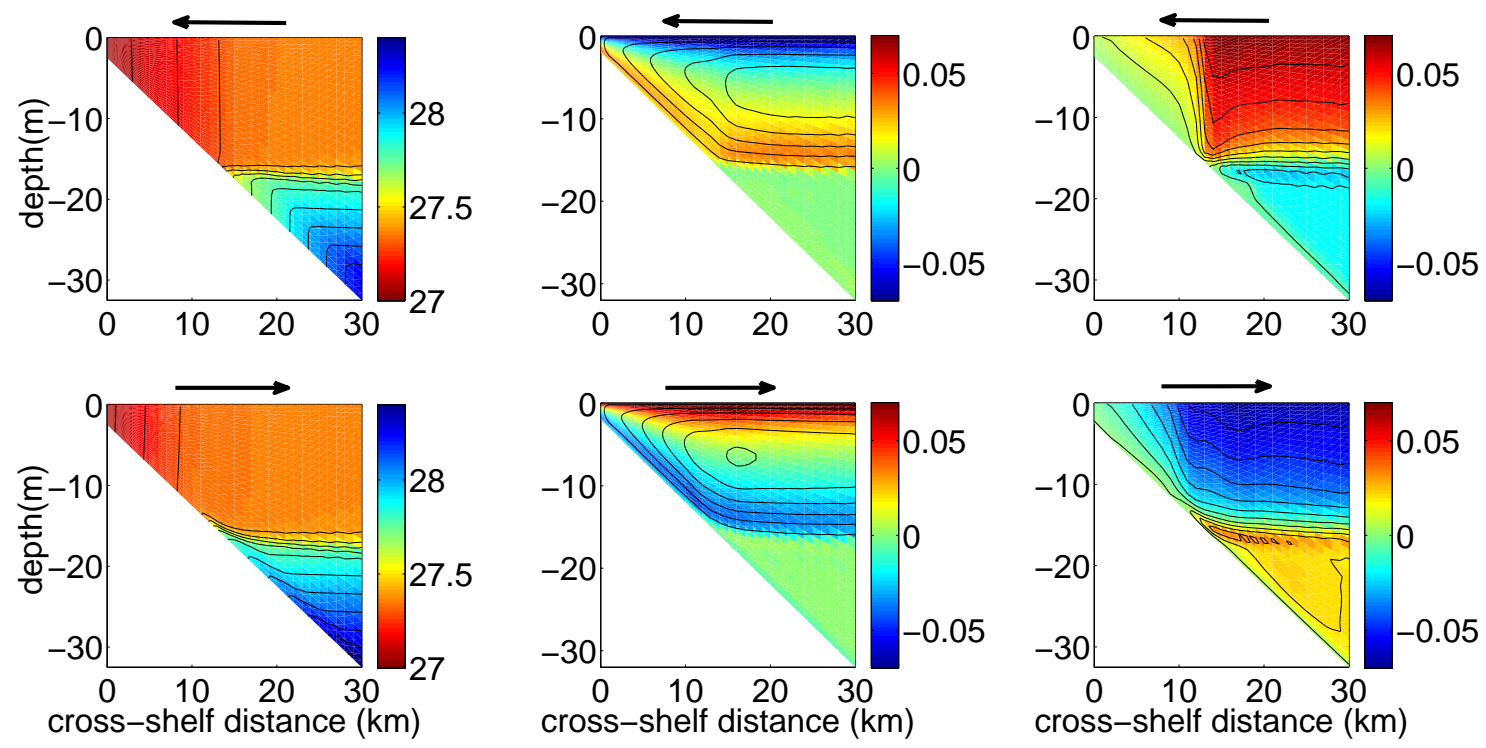
(a) $\rho\left(\mathrm{kg} / \mathrm{m}^{3}\right)$
(b) $u(\mathrm{~m} / \mathrm{s})$ and $\psi / \frac{\tau^{x}}{\rho f}$

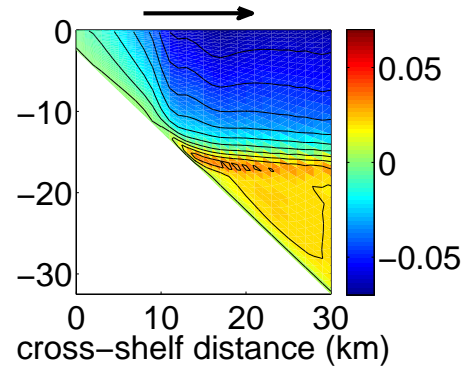

(c) $v(\mathrm{~m} / \mathrm{s})$

Figure 5-5: Inner shelf fields of density color and contours (a), cross-shelf velocity color and streamfunction contours (b), along-shelf velocity color and contours (c) resulting from onshore (top) and offshore (bottom) wind stress, indicated by arrow direction. Contour intervals are $0.1 \mathrm{~kg} / \mathrm{m}^{3}, 0.04,0.01 \mathrm{~m} / \mathrm{s}$
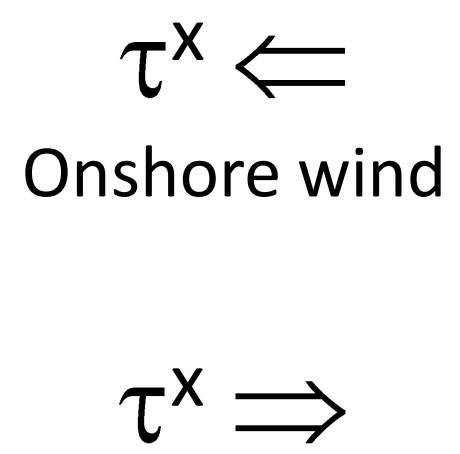

Offshore wind
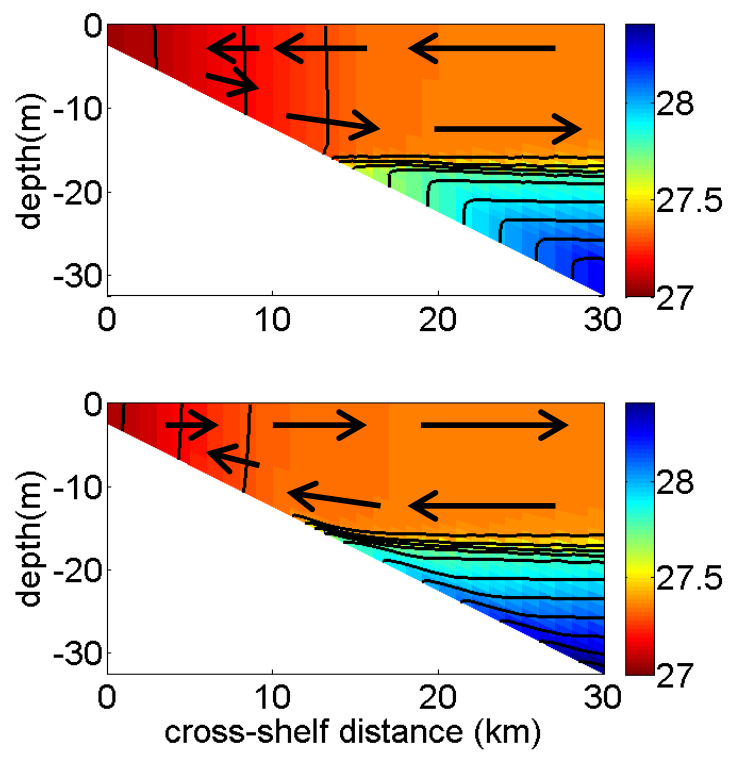

Figure 5-6: Density field resulting from onshore (top) and offshore (bottom) wind stresses on an initially stratified shelf. Arrows indicate circulation pattern. 
the bottom boundary in the neutral case. The positive stratification throughout the water column causes Richardson number of $0.05-0.01$ throughout most of the water column, causing a decreased eddy viscosity relative to the neutral density case (black lines). The resulting slight increase in shear throughout the water column allows a higher cross-shelf transport, which will be quantified in the next several sections of this Chapter.
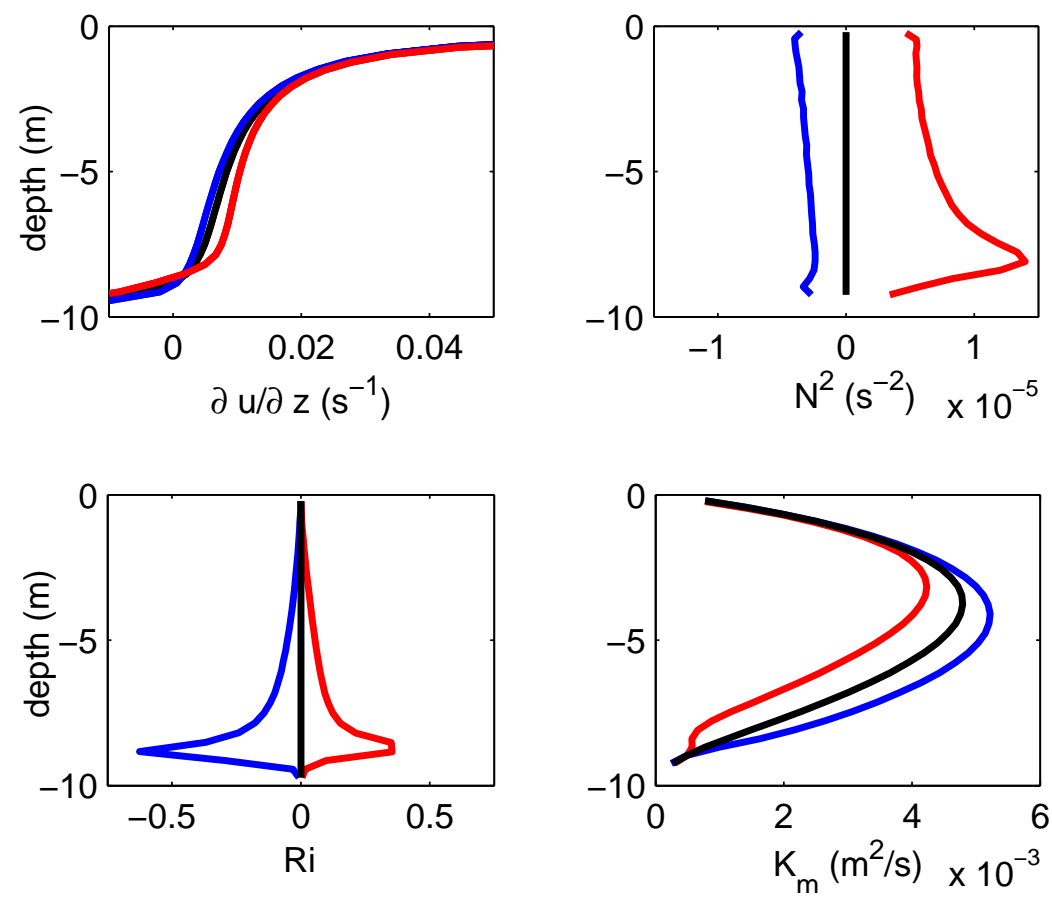

Figure 5-7: Shear $\partial u / \partial z$, stratification $\mathrm{N}^{2}$, Richardson number $\mathrm{Ri}=\mathrm{N}^{2} /(\partial u / \partial z)^{2}$, and eddy viscosity $\mathrm{K}_{m}$, resulting from onshore (blue) and offshore (red) wind stresses on an initially stratified shelf and onshore wind stress on a constant density shelf (black). Shear for onshore winds are shown as $-\partial u / \partial z$ for easier visual comparison to offshore wind

Onshore wind stress blows against the cross-shelf density gradient and advects denser water over lighter water (top row, Figure 5-6), creating a small negative stratification, which allows greater mixing and so reduces shear and transport. The reduced transport decreases the buoyancy advection that creates the negative stratification and the increased mixing also destroys the negative stratification. Both these processes bring the density profile closer to neutral. This negative feedback causes the 
buoyancy advection by onshore winds to have a smaller effect on transport than the opposite direction buoyancy advection by offshore winds. On the inner shelf, the resulting $N^{2}=-O\left(10^{-6}\right) \mathrm{s}^{-2}$, and the Richardson number is negative. Profiles of shear, stratification, Richardson number and eddy viscosity at $10 \mathrm{~m}$ water depth under an onshore wind stress on an initially stratified shelf can be see in the blue lines of Figure 5-7. The negative stratification has the largest magnitude near the surface, but the profile is nearly linear, with no mid water column maximum as for the positive stratification in offshore wind stress case. The negative Richardson number has the largest amplitude at the height of zero shear of the velocity profile, but it is the negative value throughout the water column that causes a higher eddy viscosity over the neutral case. The increased mixing forces lower shear throughout the lower two thirds of the water column, caused reduced transport compared to the neutral density or stratified, offshore wind stress cases.

Offshore of the inner shelf, the surface boundary layer depth, $\delta$, is limited by the stratification below it. For cases with non-zero initial stratification, we find the boundary layer thickness scaling of $\delta=2.3(\tau / \rho)^{\frac{1}{2}} /\left(f\left(1+N^{2} / f^{2}\right)^{\frac{1}{4}}\right)$, suggested by Tilburg (2003) fits our data well. The reduced boundary layer thickness (as compared to the unstratified case) limits the cross-shelf extent to which inner shelf dynamics dominate and also limits the deep water value that the cross-shelf transport approaches. In water depth greater than $\delta$, near full along-shelf Ekman transport to the right of the wind stress is realized regardless of stratification, but the transport parallel to the wind is sensitive to the vertical mixing, which is affected by both the wind stress strength and initial stratification.

As a result of the reduced or enhanced mixing allowed by the small final stratification, initial stratification increases $U /\left(u_{*} h\right)$ for offshore wind stress and decreases $U /\left(u_{*} h\right)$ for onshore wind stress compared to the uniform density cases. The effect of varying initial stratification is addressed in section 5.3.2. The cross-shelf velocity profiles driven by offshore wind stress are more sheared than those driven by onshore wind stress. This difference in shape is consistent with having less mixing when the 
water column is stably stratified and more mixing when it is unstably stratified. On an initially stratified shelf, we find the $u_{*}$ velocity scale and the $u_{*} h$ transport scale are robust to first order, but the relationship varies slightly with initial stratification and $u_{*}$.

\section{Varying cross-shelf wind stress magnitude}

For an inner shelf with initial stratification, varying the wind stress magnitude affects the cross-shelf transport in the same way as for the unstratified cases. Figure 5-8 shows transport above the first zero crossing of velocity in $10 \mathrm{~m}$ water depth for four different wind stress magnitudes $(\triangle$, or $\circ$ for base case), all on a shelf initialized with $N^{2}=4.25 \times 10^{-4} \mathrm{~s}^{-2}$. Black triangles indicate the transport from the unstratified

runs shown in Figure 5-4. Offshore wind stress (red) generates a higher transport than onshore (blue), but the trend of $\mathrm{U}$ increasing with $u_{*}$ at a slope of approximately 1.3 (thin black line) appears the same for both wind directions. Transport from the base case, $\tau^{x}=0.1 \mathrm{~Pa}$, run for all locations from 5 to $21 \mathrm{~m}$ water depth are indicated by $(*)$. Varying $h$ instead of $u_{*}$ also changes transport primarily along the $U \sim u_{*} h$ relation. Onshore wind stress generates transport increasingly below $1.3 u_{*} h$ as $h$ increases towards $\delta$ and offshore wind stress generates transport increasingly above $1.3 u_{*} h$ as $h$ increases. The bumps up in the blue line of asterisks and down in the red line are where the inner shelf ends and transport by both wind stress directions approach the constant deep water value.

The variation around the $U \sim u_{*} h$ scaling is sensitive to position within the inner shelf. The red (offshore) and blue (onshore) asterisks in Figure 5-8 show transport fraction $U / u_{*} h$ increases across the inner shelf for offshore wind stress and decreases across the shelf for onshore wind stress. Increasing $\tau^{x}$ increases the deep water boundary layer thickness and so increases the width of the inner shelf at a rate proportional to $1 / \alpha$ as the contact zone between the boundary layer and bathymetry widens. The width of the region of mixing-induced $\partial \rho / \partial x$ and enhanced (offshore wind) or reduced (onshore wind) transport fraction scales with the contact zone width. The cross-shelf 


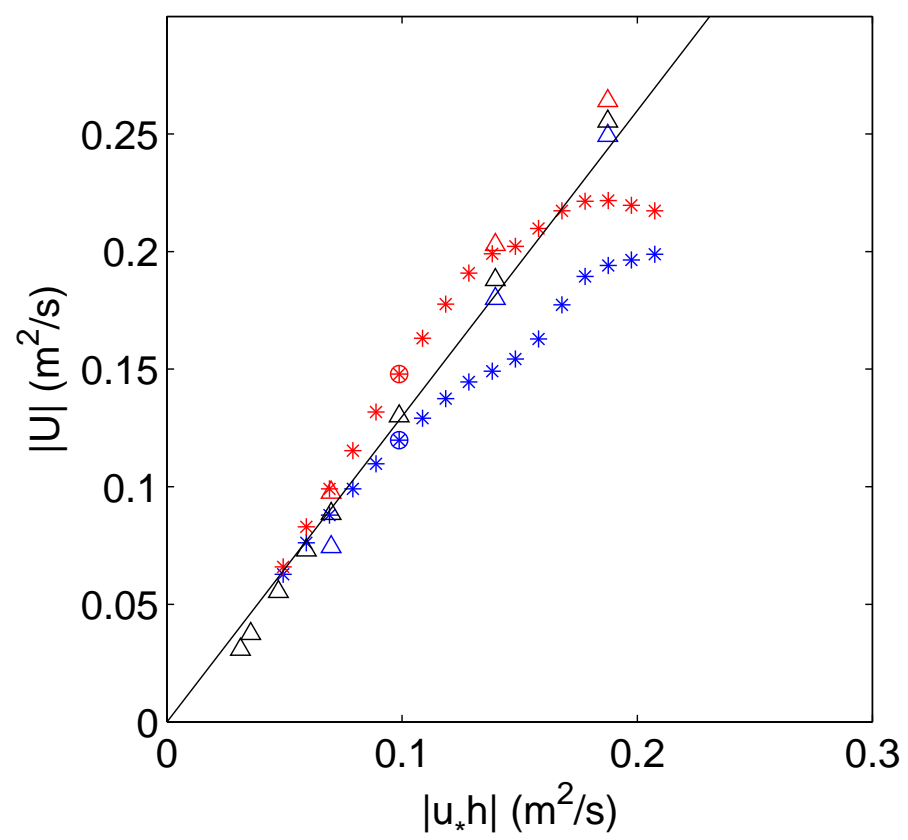

Figure 5-8: Cross-shelf transport, $U$, compared to expected inner shelf scaling, $u_{*} h$, at $h=10 \mathrm{~m}$ for initially stratified runs with onshore (blue) and offshore (red) cross-shelf wind stresses of $0.05,0.1,0.2$, and $0.36 \mathrm{~Pa}(\triangle$, with $\circ$ for base case run). Transport from $\tau^{x}=0.1 \mathrm{~Pa}$ for $h=5-21 \mathrm{~m}(*)$. Unstratified data (black) same as in Fig. 5-4. Solid line indicates slope of 1.3.

density gradient (Figure 5-9(a)) and transport fraction (Figure 5-9(b)) are shown as a function of inner shelf position, $h / \delta$, for onshore and offshore wind stresses of varying magnitudes. The density gradients fall to zero and the transport fractions approach a $1 / h$ slope where the boundary layer has separated from the bottom and the depth continues to increase while the surface density and transport are constant.

For onshore wind stress (top row of Figure 5-9), the cross-shelf density gradient is constant across the inner shelf and the gradient is well predicted by depth-averaging the initial stratification over sloping bathymetry, $\partial \bar{\rho} / \partial x=\alpha N_{0}^{2} \rho_{0} / 2 g$. The density gradient and transport magnitude transition from inner shelf to midshelf values at or slightly deeper than $h=\delta$, using $\delta=2.3(\tau / \rho)^{\frac{1}{2}} /\left(f\left(1+N^{2} / f^{2}\right)^{\frac{1}{4}}\right)$ (Tilburg, 2003).

For offshore wind stress (bottom row of Figure 5-9), upwelled water makes the outer edge of the inner shelf denser than for onshore wind (as can be seen in 5-5(a)), 
creating a higher cross-shelf density gradient very near the coast. The coastal value is constant over varying wind stresses, but the value is not uniform across the shelf. The density gradient decreases away from the coast and abruptly drops to zero at the location of the upwelling, which is consistently onshore of the location $h=\delta$. The relationship between cross-shelf density gradient and transport is shown later in Figure 5-15 in Section 5.4.2 where we elaborate on the effect of a cross-shelf density gradient.
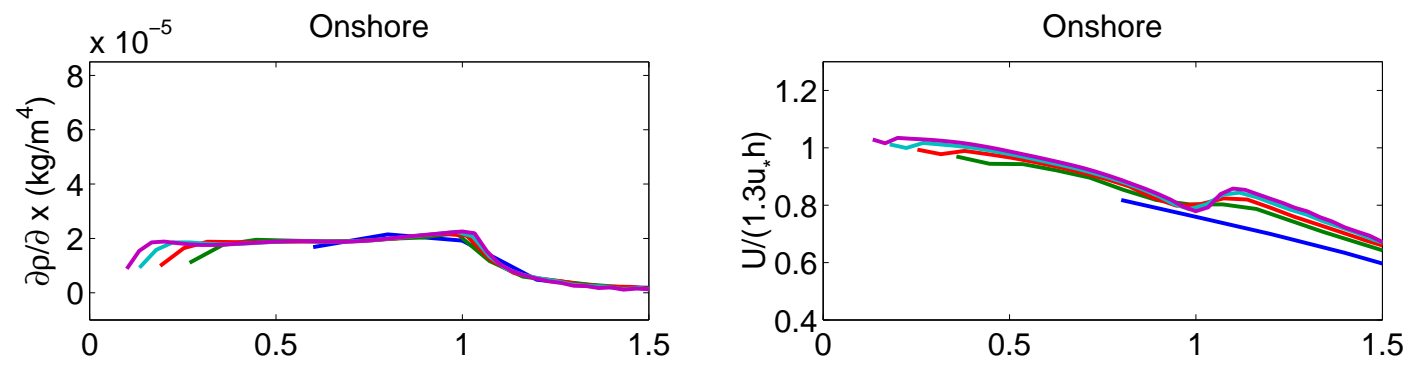

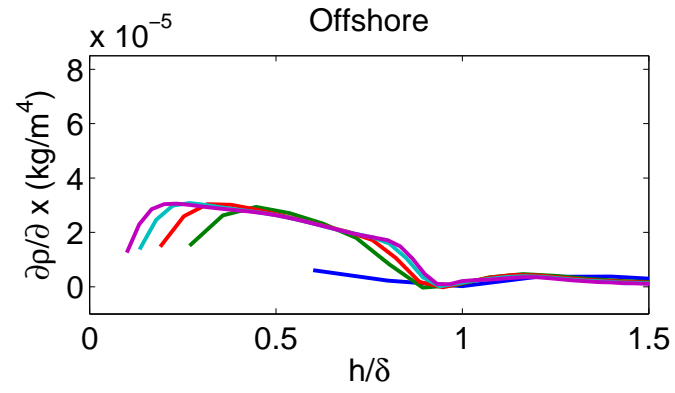

(a) cross-shelf density gradient

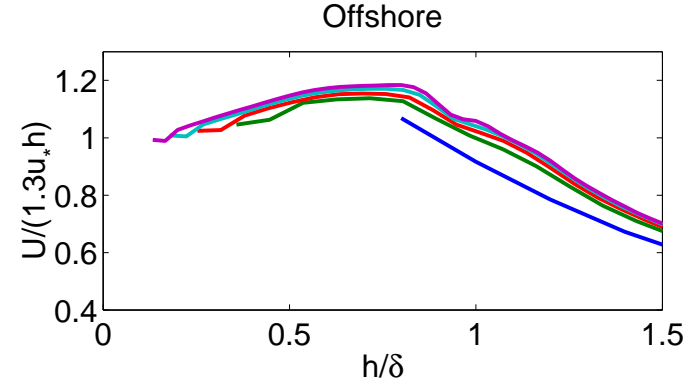

(b) transport fraction

Figure 5-9: Cross-shelf density gradient (a) and scaled transport (b) as a function of fraction boundary layer depth, $h / \delta$, generated by onshore (top) and offshore (bottom) wind stresses of $0.01,0.05,0.1,0.2$, and $0.36 \mathrm{~Pa}$ (blue, green, red, teal, and purple lines) on a shelf initially stratified with $N^{2}=4.25 \times 10^{-4} \mathrm{~s}^{-2}$.

\section{Varying initial stratification}

The strength of the initial stratification affects the inner shelf in two ways: it limits the deep water boundary layer thickness and increases the cross-shelf density gradient generated by vertical mixing over sloping bathymetry. A reduced boundary layer thickness reduces the inner shelf width and decreases transport fraction $U / u_{*} h$ as it 
moves a given depth, $h$, towards the deeper side of the $0<h<\delta$ range, as described in section 5.3.1. The density gradients fall to zero and the transport fractions approach a $1 / h$ slope where the surface boundary layer has separated from the bottom.
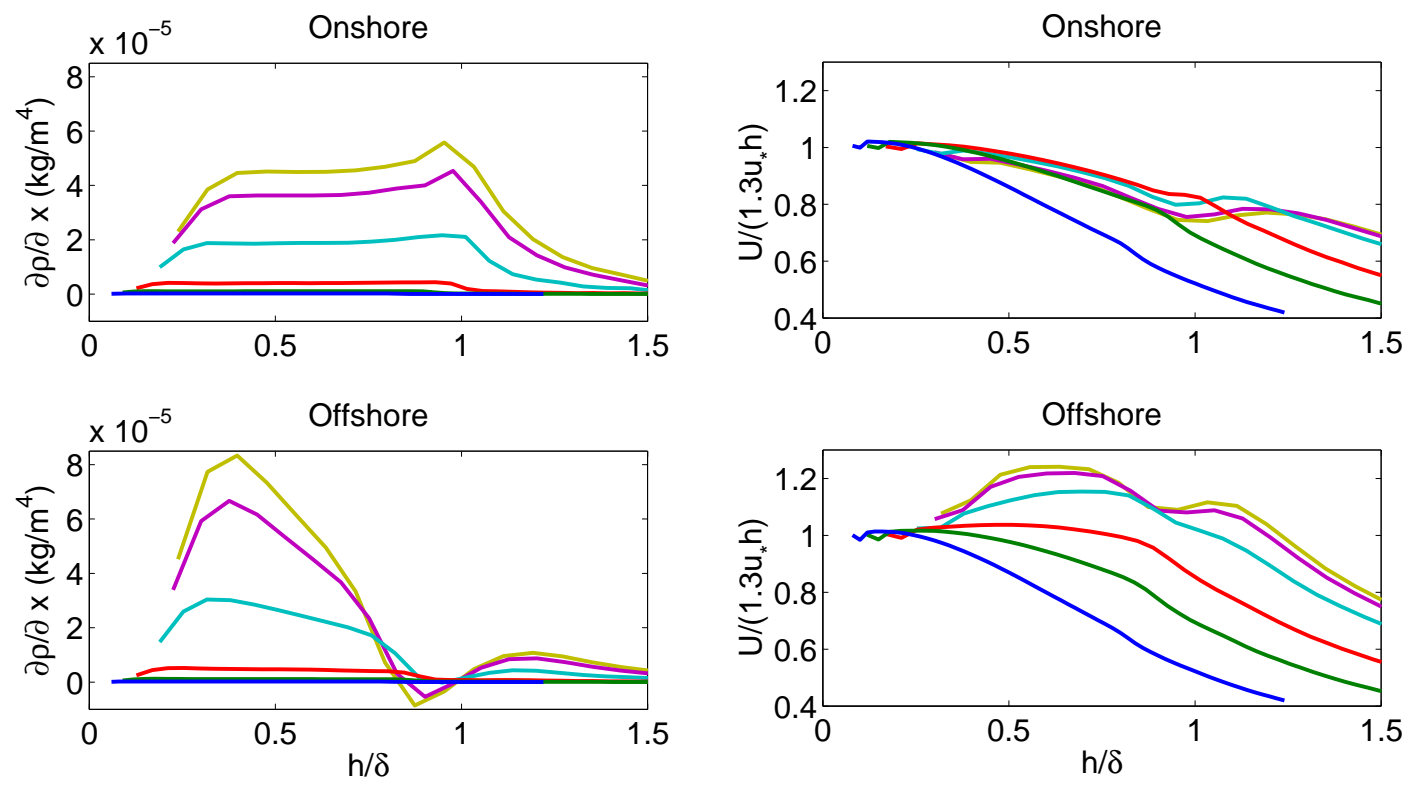

(a) cross-shelf density gradient

(b) transport fraction

Figure 5-10: Cross-shelf density gradient (a) and scaled transport (b) as a function of fraction boundary layer depth, $h / \delta$, generated by onshore (top) and offshore (bottom) $0.1 \mathrm{~Pa}$ wind stress over initial stratification of $N^{2}=(0.0425,0.2125,0.85,4.25,8.5$, and 10.625$) \times 10^{-4} \mathrm{~s}^{-2}$ (blue, green, red, teal, purple, and yellow lines).

An increased cross-shelf density gradient is expected to increase buoyancy advection for a given strength of circulation. For an offshore wind stress, the increased buoyancy advection will increase stratification and increase transport fraction by raising the shear needed to cause instability. For an onshore wind stress, the increased buoyancy advection will decrease stratification and decrease the transport fraction by lowering the shear needed to cause instability. Figure 5-10 shows the cross-shelf density gradient and transport fraction for onshore and offshore wind stresses over inner shelves with six different initial stratifications.

For onshore wind stress (top row of Fig. 5-10), higher initial stratification increases the cross-shelf density gradient uniformly across the width of the inner shelf. The 
cross-shelf density gradient is predicted by $\partial \bar{\rho} / \partial x=\alpha N_{0}^{2} \rho_{0} / 2 g$, as can be seen on the right side of Figure 5-11. The higher cross-shelf density gradient generated by higher initial stratification allows a larger (more negative) unstable stratification to be produced by the wind-driven advection. The left side of Figure 5-11 shows the final stratification at $10 \mathrm{~m}$ water depth over the range of initial stratifications. Note that the scale of the vertical axis is 100 times smaller than the scale of the horizontal axis. For onshore wind stress, Figure 5-10(b) shows similar reductions in transport fraction across the width of the inner shelf for varying initial stratification. The increase in density gradient and reduction of shelf width both act to reduce the transport fraction at a particular location. The blue symbols in Figure 5-12 demonstrate a consistent trend of decreased transport for increased initial stratification at $10 \mathrm{~m}$ water depth.

For offshore wind stress, the cross-shelf density gradient (bottom of Fig. 5-10(a)) increases with increasing initial stratification and the maximum $\partial \rho / \partial x$ value, located just a few kilometers from shore, is higher than that predicted purely by vertical mixing of initial stratification. At a given location on the inner shelf, the cross-shelf density gradient increases linearly with initial stratification (red symbols in Figure 511) but the slope of this line will depend heavily on the particular location within the inner shelf. A higher cross-shelf density gradient creates a higher local stratification (Figure 5-11) by a given amount of wind-driven advection. The higher stratification allows a greater shear and transport to be maintained before the positive feedback process is limited by mixing. The transport fraction (bottom of Fig. 5-10(b)) increases with higher initial stratification, indicating that the increase in transport fraction with initial stratification at $10 \mathrm{~m}$ water depth (red symbols in Figure 5-12) is due to advection of the elevated cross-shelf density gradient rather than the decrease that might be caused by a higher $h / \delta$ ratio.

\subsubsection{Section summary}

To first order $u / u_{*}$ scales with $z / h$ and $U$ scales with $u_{*} h$ for both stratified and unstratified cases. These relationships vary with $N^{2}$ and $\delta / h$. Transport varied by 

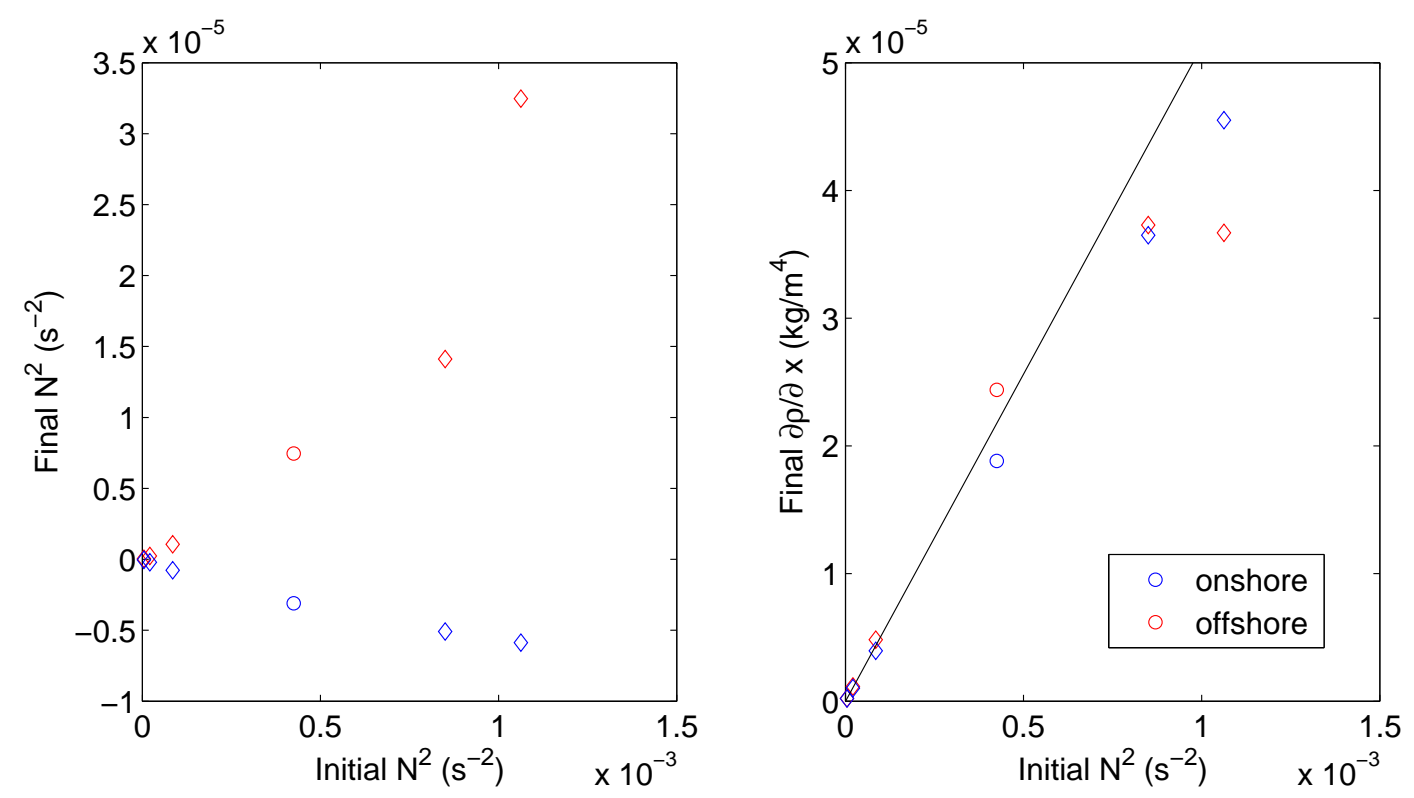

Figure 5-11: Final stratification (left) and cross-shelf density gradient (right) as a function of initial stratification at $10 \mathrm{~m}$ water depth. Black line indicates $\partial \bar{\rho} / \partial x=$ $\alpha N_{0}^{2} \rho_{0} / 2 g$

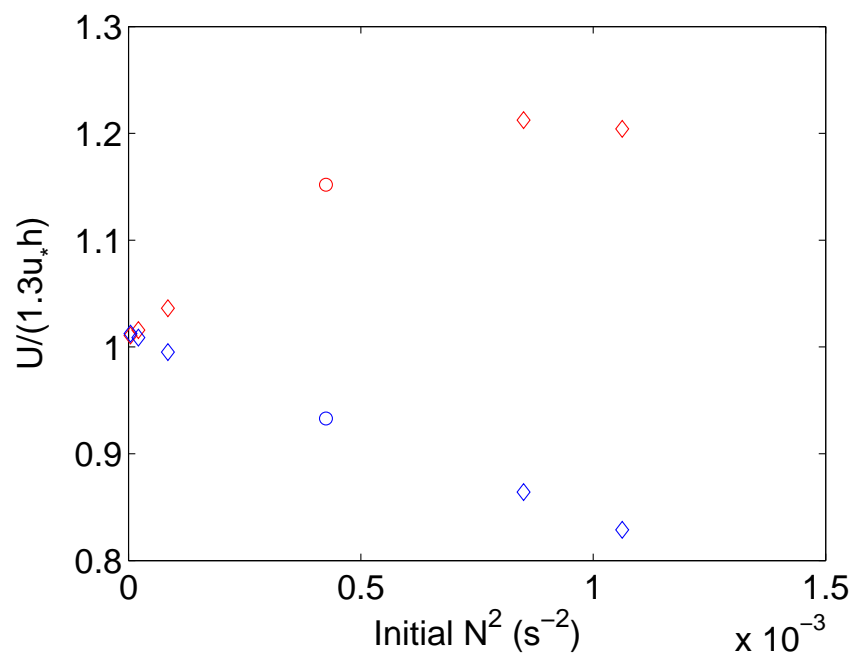

Figure 5-12: Transport fraction as a function of initial stratification at $10 \mathrm{~m}$ water depth for onshore (blue) and offshore (red) wind stresses

$30 \%$ and $50 \%$ respectively for parameter ranges of these experiments, which are typical of the inner shelf south of Martha's Vineyard, MA. In these initial experiments, transport varied much more over a typical range of wind stresses than initial stratifications. However, the final stratification that could increase or decrease transport 
by $30 \%$ was smaller than any we could measure in the SWWIM field program, indicating the importance of understanding the mechanisms that create and maintain stratification on the inner shelf.

\subsection{The cross-shelf density gradient}

In the previous experiment varying initial shelf stratification, the resulting cross-shelf density gradient was a critical component in setting the balance between circulation and mixing. Now we impose a range of both positive and negative cross-shelf density gradients while keeping the initial stratification constant. This experiment aims to simulate the response to cross-shelf wind forcing in the presence of a cross-shelf density gradient. South of Martha's Vineyard, MA and on many shelves, there is a crossshelf density gradient due to a salinity gradient in addition to the density gradient resulting form vertical mixing of initial stratification. We ask how the cross-shelf transport changes as a results of the imposed horizontal density gradient.

This kind of cross-shelf gradient may remain after wind has mixed and perhaps momentarily lulled because our wind stress here (as for all our experiments) starts at zero and increases over the first two days. The model is initialized with no initial velocity, but with a surface tilt to balance the cross-shelf pressure gradient that results from the initial cross-shelf density gradient. The surface elevation is chosen to make the level of no motion at half the water depth so $\bar{u} \approx 0$. A thermal wind shear is spun up in the first inertial period of the model run. Except for one run with horizontal isopycnals, the initial conditions used here all have constant isopycnal slopes greater than or equal to bottom slope so the cross-shelf gradient of the depthaveraged density is the same sign as the imposed cross-shelf density gradient. If isopycnals were initialized with a slope less than one half the bottom slope, vertical

mixing would create a positive cross-shelf density gradient regardless of the sign of the initial cross-shelf density gradient.

Isopycnals were initialized as uniformly diagonal with cross-shore and vertical 
temperature gradients that were representative of summertime near MVCO. Middle Atlantic Bight climatologies of temperature and salinity (Lentz, 2008) and density (Zhang et al., 2011) indicate that uniformly sloped isopycnals are representative of the seasonal mean near Martha's Vineyard. All runs in this section were initialized with $N^{2}=1.70 \times 10^{-4} \mathrm{~s}^{-2}$. Cross-shelf density gradients are $( \pm 1.75, \pm 3.49$, and $\pm 8.73) \times 10^{-5} \mathrm{~kg} / \mathrm{m}^{4}$. Figure $5-13$ shows the initial density fields at both coasts for one value of the cross-shelf density gradient.
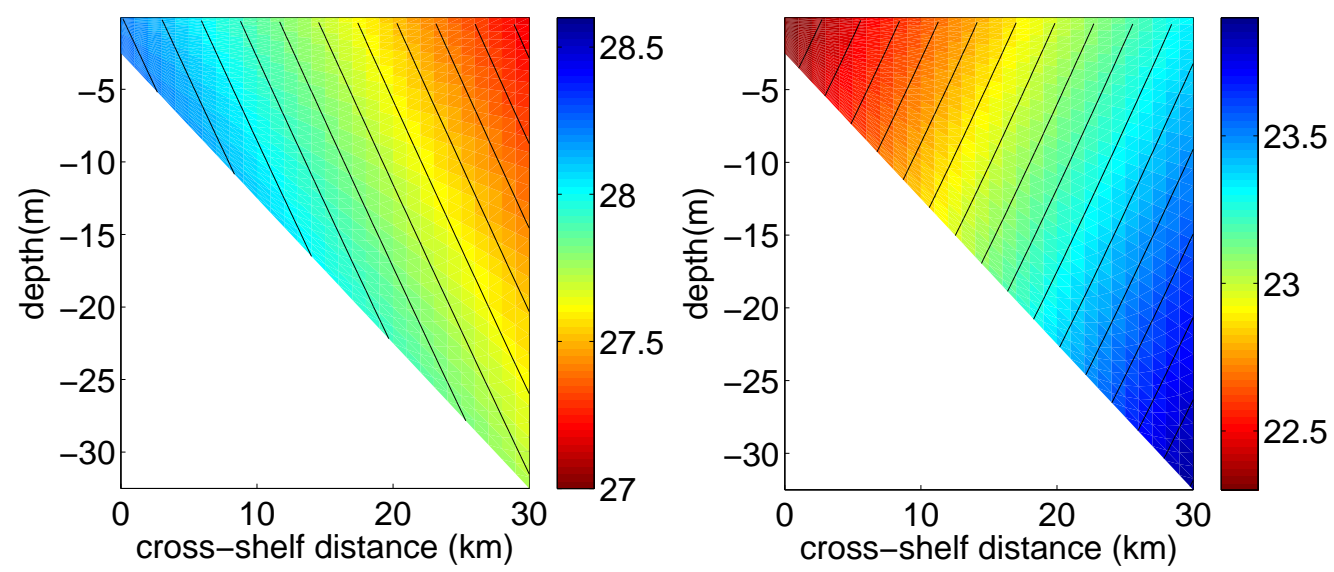

Figure 5-13: Cross-shelf sections of initial density field for an initial cross-shelf density gradient of $\pm 3.5 \times 10^{-5} \mathrm{~kg} / \mathrm{m}^{4}$ and initial vertical density gradient of $1.75 \times 10^{-2} \mathrm{~kg} / \mathrm{m}^{4}$ $\left(N^{2}=1.7 \times 10^{-4} \mathrm{~s}^{-2}\right)$. Runs with the same stratification but higher, lower, and zero cross-shelf density gradients (not show) were also part of this experiment.

\subsubsection{Predicting the cross-shelf density gradient}

Complete vertical mixing of spatially uniform vertical and horizontal density gradients over a sloping bottom will generate a cross shelf density gradient of

$$
\left(\frac{\partial \rho}{\partial x}\right)_{f i n a l}=-\frac{\alpha}{2}\left(\frac{\partial \rho}{\partial z}\right)_{0}+\left(\frac{\partial \rho}{\partial x}\right)_{0}
$$

Over the inner shelf, wind mixing affects the entire water column and the final crossshelf density gradients closely match those predicted from initial conditions. Figure 5-14 shows the final cross-shelf density gradients calculated using the surface density 
values at $10 \mathrm{~m}$ water depth (8 $\mathrm{km}$ from shore) compared to the gradients predicted by equation 5.11. The figure includes model runs from wind stress magnitude, stratification, and cross-shelf density gradient varying experiments for all runs where $10 \mathrm{~m}$ water depth is within the inner shelf based on transitions shown in Figures 5-9 and $5-10$.

Overall, the prediction holds well over the wide range of cross-shelf density gradients shown in Figure 5-14(a). Density gradients at the end of the 5 day model runs match the prediction made for purely vertical mixing regardless of whether the density gradient was initially imposed, or created by mixing of initial stratification. Figure 5-14(b) shows the boxed subset region of 5-14(a) in greater detail. For the data that have variation in wind stress magnitude $(\triangle)$ and 5 to $12 \mathrm{~m}$ water depth $(\cdot)$ but the same initial stratification, the predicted cross-shelf density gradient is the same, while the modeled gradients vary slightly around that prediction because the prediction for $\partial \rho / \partial x$ in equation 5.11 does not depend on wind magnitude or water depth. The offshore (red) data in this subset show a greater spread than the onshore (blue) data because offshore wind stress creates variation in the cross-shelf density gradient across the inner shelf that is not a function of purely vertical mixing, while the onshore wind stress creates a nearly constant density gradient across the shelf, as demonstrated in Figure 5-9(a).

It should be noted that the imposed initial cross-shelf density gradients do slump slightly as the thermal wind balance spins up. The adjustment slightly increases the stratification throughout the domain and this stratification does limit the deep water boundary layer thickness. However, these changes to stratification and boundary layer thickness are much smaller than the range of stratifications and boundary layer thicknesses generated by the set of runs with varying initial stratification, while at the same time spanning a much larger range of cross-shelf density gradients than those created by the mixing of the initially stratified runs. 


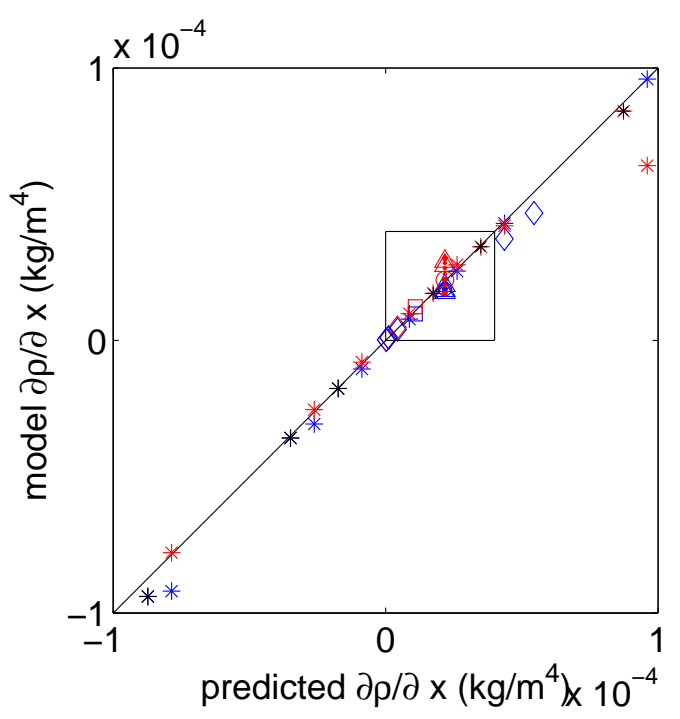

(a) All data

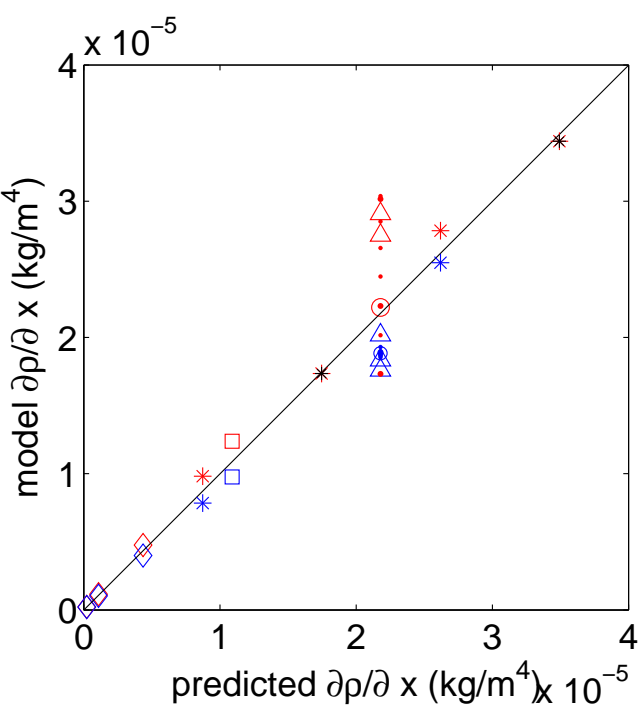

(b) Subset

Figure 5-14: (a) $\partial \rho / \partial x$ from the model using $\rho$ from the surface at $10 \mathrm{~m}$ water depth ( $8 \mathrm{~km}$ from shore) compared to $\partial \rho / \partial x$ predicted by purely vertical mixing of initial isopycnals. Base case run (o) and runs varying $N_{0}^{2}(\diamond), \tau_{s}^{x}(\triangle), \alpha(\square)$, depth $(\cdot)$, and initial cross shelf density gradient $(*)$ with onshore (blue), offshore (red), or zero (black) wind stress. (b) Expanded axes for area indicated by box in (a)

\subsubsection{The effect of a cross-shelf density gradient on transport}

We expect a cross-shelf density gradient to modify the wind-driven transport by changing the values of shear and stratification that close our governing equations. When wind-driven circulation advects lighter over denser water, it creates a small positive stratification, which reduces mixing that in turn allows greater vertical shear in the cross-shelf velocity and greater cross-shelf transport. In contrast, when winddriven circulation advects denser over lighter water, mixing is enhanced and shear and transport are reduced. To find a modification of the $u_{*} h$ scale for transport, we first look for the relevant scales by which to measure the cross-shelf density gradient. Scaling the cross-shelf density gradient by the background density and gravity creates a cross-shelf gradient of reduced gravity, $(g / \rho)(\partial \rho / \partial x)$. The stratification created by advecting the cross-shelf density gradient will be proportional to this value. The shear in the flow, $\partial u / \partial z \sim u_{*} / h$, contributes to how much buoyancy is advected horizontally to create stratification by a given $\partial \rho / \partial x$, but also drives the instabilities that mix 
momentum and density. Based on these two scales, we create a nondimensionalized cross-shelf density gradient as the ratio of the reduced gravity to the square of the shear in the flow. The value takes a form similar to a horizontal Richardson number, also called the Simpson number (Simpson et al., 1990), which in estuarine contexts is used to describe the ratio of the horizontal density gradient (stratifying force) to vertical mixing (destratifying force).

Equation 5.12 defines a modified horizontal Richardson number, $R i_{x}$, that we use as our nondimensionalized form of the cross-shelf density gradient. The sign of the wind stress, $\tau^{x} /\left|\tau^{x}\right|$, has been added to distinguish between advection that will create positive stratification, partially limiting the mixing effect of the wind stress, and that which will create negative stratification, enhancing mixing.

$$
\operatorname{sign}\left(\tau^{x}\right) R i_{x}=\frac{\tau^{s x}}{\left|\tau^{s x}\right|} \frac{g}{\rho} \frac{h^{2}}{u_{*}^{2}} \frac{\partial \rho}{\partial x}
$$

Figure 5-15 compares transport fraction with the signed horizontal Richardson number. In 5-15(a), the density gradient used to calculate $R i_{x}$ is from the final state of the model and in 5-15(b), the density gradient used to calculate $R i_{x}$ is predicted from the initial conditions by equation 5.11. These plots show clearly the complimentary relationship between Richardson number and transport fraction. For density values increasing in the direction of the wind stress wind stress, an increased density gradient increases transport by increasing stratification and reducing mixing. For a wind stress opposing the density gradient, a larger cross-shelf density gradient decreases the transport fraction by creating more mixing for a particular wind stress magnitude. The steeper slope of the transport for positive $\operatorname{sign}\left(\tau^{s x}\right) R i_{x}$ values than negative ones indicates the transport's stronger response to wind blowing with a given cross-shelf density gradient (from lighter towards denser water) than against it. The larger change in transport for offshore than onshore wind stresses in section 5.3.2 was not just an effect of the higher near shore density gradient created by offshore wind stresses, but an effect of the two directions of wind stress acting in different ways on 
the cross-shelf density gradient.

The higher slope observed in Figure 5-15 for positive values of the horizontal Richardson number is similar to the response of wind shear to flux Richardson number as described by Monin-Obukhov (1954) similarity theory and demonstrated with atmospheric field measurements by Businger et al. (1971). The flux Richardson number indicates the balance between buoyancy production and shear production of turbulent kinetic energy (Flierl and Ferrari, 2006). A positive flux Richardson number indicates the stratification is positive and turbulent kinetic energy is lost to potential energy. A negative flux Richardson number indicates a negative stratification is generating turbulence. Large positive $(>1)$ flux Richardson numbers indicate turbulence is suppressed by the stratification and transport can increase continuously with increased forcing. Large negative $(<-1)$ flux Richardson numbers indicate strong convective instability, where increased wind stress forcing will not increase shear and transport because the convective mixing will destroy the vertical shear in the velocity. For small flux Richardson numbers $\left(-1<R i_{f}<1\right)$, the system is weakly stratified and shear production of turbulence dominates over buoyancy production of turbulence. In our model, advection of the horizontal density gradient creates either a positive or negative stratification, which determines the sign of the flux Richardson number. This relationship between the horizontal and vertical density gradients allows the use of the predictable cross-shelf density gradient in describing the transport efficiency. The different slopes of the transport - density gradient relationship evident for positive and negative values of the horizontal Richardson number in Figure 5-15 are caused by the different states of the system indicated by positive and negative (flux) Richardson numbers.

This result is particularly important for planning field investigations and interpreting data because the cross-shelf density gradients analyzed here, along with wind stresses and water depths, are measurable with common observational techniques. In contrast, the small stratification resulting from horizontal buoyancy advection across the inner shelf, as described in section 5.3.2 and shown in Figure 5-11 are not readily 


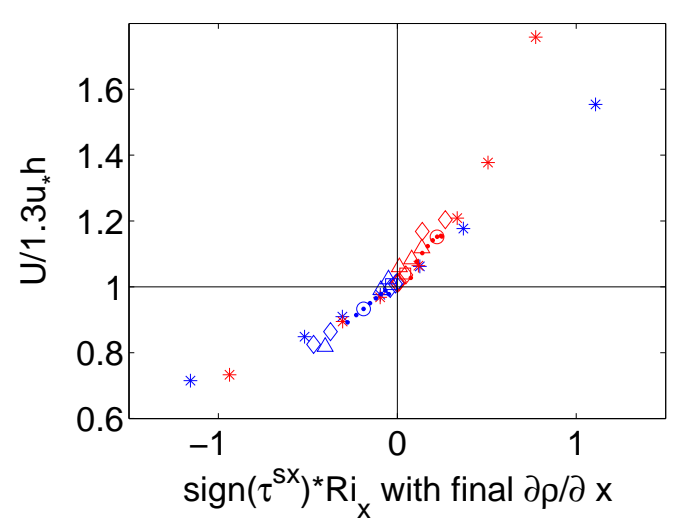

(a) Using model $\partial \rho / \partial x$

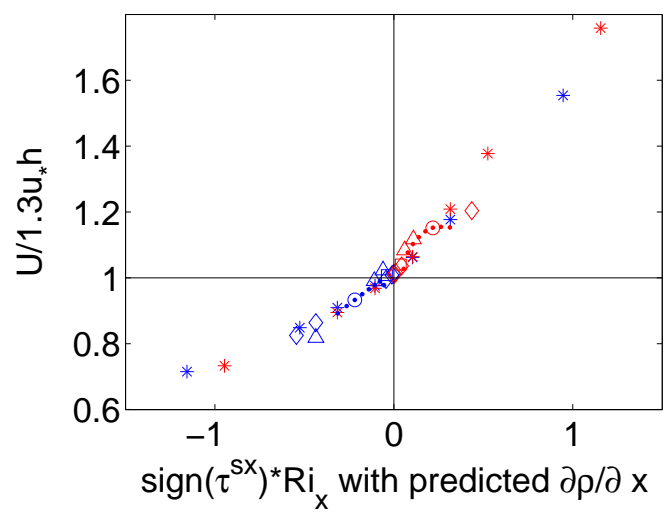

(b) Using predicted $\partial \rho / \partial x$

Figure 5-15: Transport fraction as a function of cross-shelf density gradient. Base case run (०) and runs varying $N_{0}^{2}(\diamond), \tau_{s}^{x}(\triangle), \alpha(\square)$, depth $(\cdot)$, and initial cross shelf density gradient $(*)$ with onshore (blue), offshore (red) wind stress.

measurable from the data set used in Chapters 2 - 4 of this theses, or from other similar moored observations.

\subsection{Surface Heat Flux}

So far, this model has been used to demonstrate how both initial stratification and an initial cross-shelf density gradient affect the density gradient across the inner shelf and the cross-shelf transport. Both these experiments tested the effects of changing the initial conditions existing prior to the onset of wind forcing. Here we ask, what is the effect of a continuous surface heat flux on the wind-driven system? The model setup is the same as was described for the base case in section 5.2.2 with the addition of a constant surface heat flux, $Q\left(\mathrm{~W} / \mathrm{m}^{2}\right)$. Surface heat flux values of $-10,0,10,50$, 100,150 , and $200 \mathrm{~W} / \mathrm{m}^{2}$ are used. In the model the surface heat flux is imposed as a temperature flux through the surface, $Q / \rho c_{p}\left({ }^{\circ} \mathrm{C} \frac{\mathrm{m}}{\mathrm{s}}\right)$, using a seawater specific heat capacity, $c_{p}=3985 \frac{\mathrm{J}}{\mathrm{kg}^{\circ} \mathrm{C}}$. The values for $Q$ used here are representative of summertime daily averages of net surface heat flux over the inner shelf off Martha's Vineyard, MA, including latent, sensible and radiative fluxes. In reality the latent heat flux primarily cools the surface through evaporation while the incoming radiation heats the water 
column as a function that decays away from the surface at a rate proportional to one over the wavelength. Long and shortwave radiation decay coefficients for the open ocean surface mixed layer, such as those used by Price et al. (1986), are unlikely to apply directly to the inner shelf where the water is often turbid and absorption is more likely to happen very near the surface. We also recognize the unrealistic nature of surface heating without the accompanying evaporative cooling that would destabilize the near surface region, but due to the strong wind mixing that extends through the full water depth on the inner shelf, we do not expect this omission of an additional destabilizing force to substantially alter our results. With awareness of these simplifications of the surface heating process, we will not expect the vertical distribution of properties provided by the model to be completely realistic and will focus our analysis on depth-averaged values.

While this experiment was originally conceptualized as a way to test if a surface heat flux could create a stratified inner shelf, a brief consideration of the flux Richardson number, the ratio of buoyancy flux to shear production, over typical ranges of inner shelf surface heat flux and wind stress values demonstrates why this model implementation is unlikely to create the stratification we initially sought. The flux Richardson number is defined

$$
R i_{f}=\frac{\text { buoyancy flux }}{\text { shear production }}=\left(\frac{g \alpha_{T} Q}{\rho c_{p}}\right)\left(\frac{\tau}{\rho} \frac{\partial \bar{u}}{\partial z}\right)^{-1}
$$

where, as we have already discussed, the appropriate scalings are $\tau / \rho \sim u_{*}^{2}$ and $\partial \bar{u} / \partial z \sim u_{*} / h$, leaving us with

$$
R i_{f}=\frac{g \alpha_{T} Q}{\rho c_{p}} \frac{h}{u_{*}^{3}}
$$

In these experiments we use surface heat flux values from -10 to $200 \mathrm{~W} / \mathrm{m}^{2}$ all with $\tau=0.1 \mathrm{~Pa}$, and additionally test 50 and $100 \mathrm{~W} / \mathrm{m}^{2}$ with $\tau=0.05 \mathrm{~Pa}$. These heat flux and wind stress values give us a range of $-0.04<R i_{f}<0.85$ for all except the 
$0.05 \mathrm{~Pa}, 100 \mathrm{~W} / \mathrm{m}^{2}$ run, which has $R i_{f}=1.8$. These flux Richardson values approach one and indicate the flow may be stabilized by stratification, but the strongest shear is also near the surface where the surface heat flux is added, making the in situ flux Richardson number lower than the value based on the shear scale of $u_{*} / h$. Indeed, the results we observe and describe in the following section demonstrate that the surface heat flux influences the transport and the cross-shelf density gradient, but does not create stratification beyond the minimal value generated by the advection of the cross-shelf density gradient on an actively mixing inner shelf.

\subsubsection{Surface heat flux creates a cross-shelf density gradient}

With continuous wind stress causing mixing, the incremental addition of heat through the surface never creates stratification strong enough to shut down the vertical mixing. We estimate the cross-shelf density gradient that would be created on the inner shelf by our imposed surface heat flux over the sloping bathymetry, assuming complete vertical mixing and no horizontal advection of buoyancy. After some time, $t(\mathrm{~s})$, in water depth $h(\mathrm{~m})$, the depth averaged temperature, $T\left({ }^{\circ} \mathrm{C}\right)$, will have increased by $Q t / h \rho c_{p}\left({ }^{\circ} \mathrm{C}\right)$ over the initial temperature at that location. The cross-shelf temperature gradient generated by the surface heat flux, $Q$ is given by

$$
\begin{aligned}
\left(\frac{\partial T}{\partial x}\right)_{Q} & =\frac{\partial}{\partial x}\left(\frac{Q t}{\rho c_{p} h}\right), \text { where } h=h(x) \\
& =\frac{Q t}{\rho c_{p}}\left(\frac{\partial}{\partial x} \frac{1}{h}\right) \\
& =\frac{Q t}{\rho c_{p}}\left(\frac{-\alpha}{h^{2}}\right), \text { for } h=h_{0}+\alpha x .
\end{aligned}
$$

Then the density gradient generated by the surface heat flux is

$$
\left(\frac{\partial \rho}{\partial x}\right)_{Q}=-\rho_{0} \alpha_{T} \frac{\partial T}{\partial x}=\rho_{0} \alpha_{T} \frac{Q t}{\rho c_{p}}\left(\frac{\alpha}{h^{2}}\right),
$$


where $\alpha_{T}$ is the thermal expansion coefficient in the linear equation of state. The cross-shelf density gradient created by vertically mixing the initial stratification is still

$$
\left(\frac{\partial \rho}{\partial x}\right)_{N^{2}}=-\left.\frac{\alpha}{2} \frac{\partial \rho}{\partial z}\right|_{0}
$$

so the total cross-shelf density gradient will be

$$
\frac{\partial \rho}{\partial x}=\left(\frac{\partial \rho}{\partial x}\right)_{N^{2}}+\left(\frac{\partial \rho}{\partial x}\right)_{Q}=-\left.\frac{\alpha}{2} \frac{\partial \rho}{\partial z}\right|_{0}+\rho_{0} \alpha_{T} \frac{Q t}{\rho c_{p}}\left(\frac{\alpha}{h^{2}}\right)
$$

In Figure 5-16, modeled and predicted cross-shelf density gradients are show for model runs with $Q=150 \mathrm{~W} / \mathrm{m}^{2}$ and wind stresses of $0.1 \mathrm{~Pa}(5-16(\mathrm{a}))$ or $0.05 \mathrm{~Pa}$ (5-16(b)). Blue and red lines are model output from onshore and offshore wind stresses, respectively, and black lines are the prediction based on initial stratification and input surface heat flux. The modeled cross-shelf density gradient closely follows the $1 / h^{2}$ trend predicted by equation $5.14 \mathrm{~d}$. The modeled $\partial \rho / \partial x$ sharply decreases and separates from the prediction at the water depth where the surface boundary layer separates from the bottom because the switch from a mixing depth that follows bathymetry to a constant boundary layer thickness is not part of the simplified dynamics included in the prediction.

For both wind stress magnitudes, the transition from inner shelf to a boundary layer separate from the bottom happens at a shallower depth for the offshore wind stress than for onshore. The depth of the transition is affected by the wind stress, but the magnitude of the density gradient within the inner shelf is not, as demonstrated by the close match of the numerical model output to equation $5.14 \mathrm{~d}$, which does not include the wind stress magnitude.

Figure 5-17 shows the modeled and predicted cross-shelf density gradients resulting from a range of surface heat flux values in $10 \mathrm{~m}$ water depth after 5 days. Blue and red symbols are model output from onshore and offshore wind stresses, respec- 


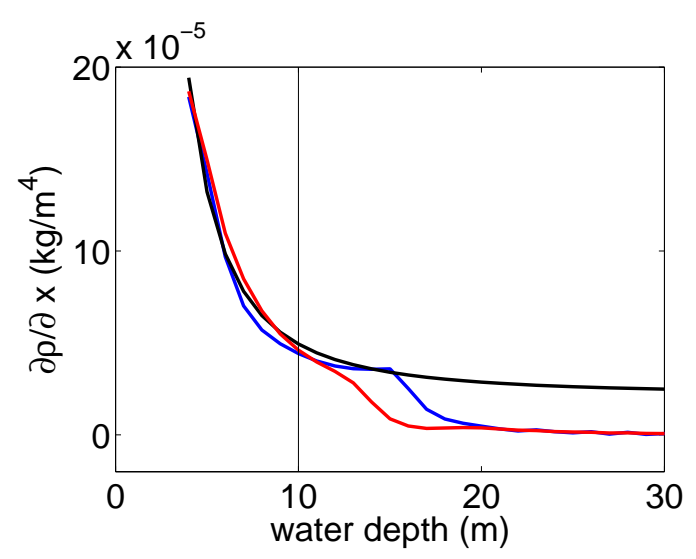

(a) $Q=150 \mathrm{~W} / \mathrm{m}^{2}, \tau=0.1 \mathrm{~Pa}$

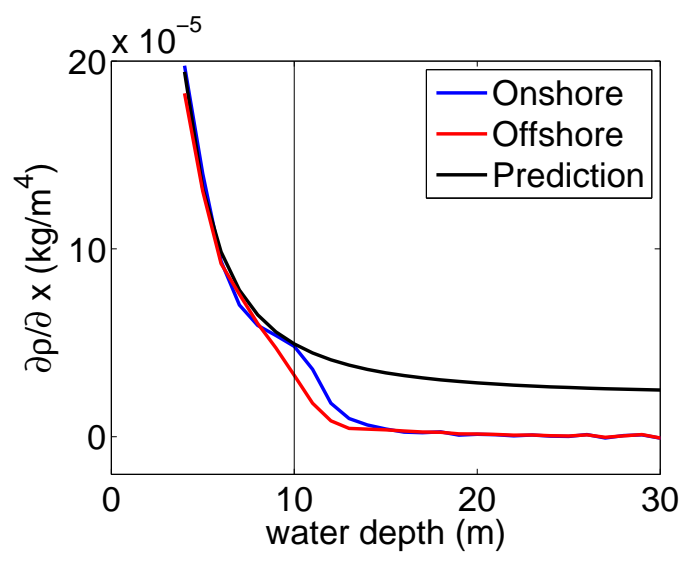

(b) $Q=150 \mathrm{~W} / \mathrm{m}^{2}, \tau=0.05 \mathrm{~Pa}$

Figure 5-16: Cross-shelf variation of $\partial \rho / \partial x$ as a function of water depth for onshore (blue) and offshore (red) wins stresses and as predicted by equation 5.14d. Vertical black lines indicate the $10 \mathrm{~m}$ deep locations used in Figure 5-17

tively. The black line connects predictions made by equation 5.14d from the initial stratification and input surface heat flux.

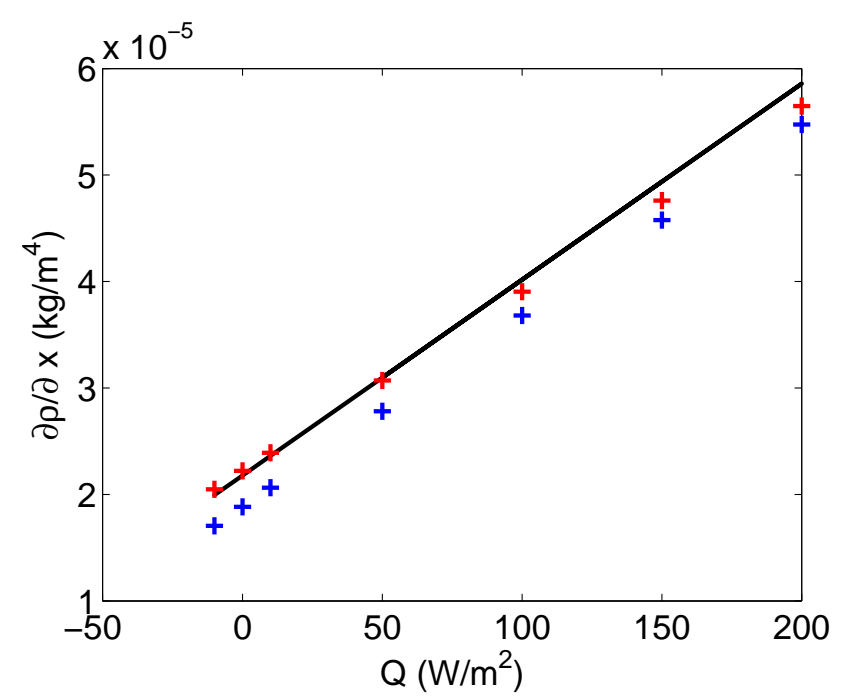

Figure 5-17: Cross-shelf density gradient after surface heating $(+)$ and $0.1 \mathrm{~Pa}$ wind stresses for onshore (blue), offshore (red) wind directions and as predicted for complete vertical mixing (black line) by equation 5.14.

For both on and offshore wind directions, the cross-shelf density gradient increases with $Q$, closely following the linear trend predicted by equation 5.14. The close match of $\partial \rho / \partial x$ here with equation 5.14 and in section 5.4.1 with equation 5.11 
indicates that the cross shelf density gradient is set primarily by one-dimensional processes on the inner shelf. The noted deviations from theses predictions indicate that advection becomes important in setting the density structure for offshore wind stress, particularly near the outer edge of the inner shelf.

\subsubsection{Surface heat flux affects cross-shelf transport}

\section{Transport as a function of $Q$}

Surface heat flux changes the cross-shelf transport by increasing the cross-shelf density gradient that results from vertical mixing. The linear relationship $\partial \rho / \partial x$ has with $Q$ translates to a near linear change in transport with $Q$, for a particular water depth and wind stress magnitude and direction. Figure 5-18 shows the trend of increasing transport by offshore wind stress and decreasing transport by onshore wind stress for increasing surface heat flux values.

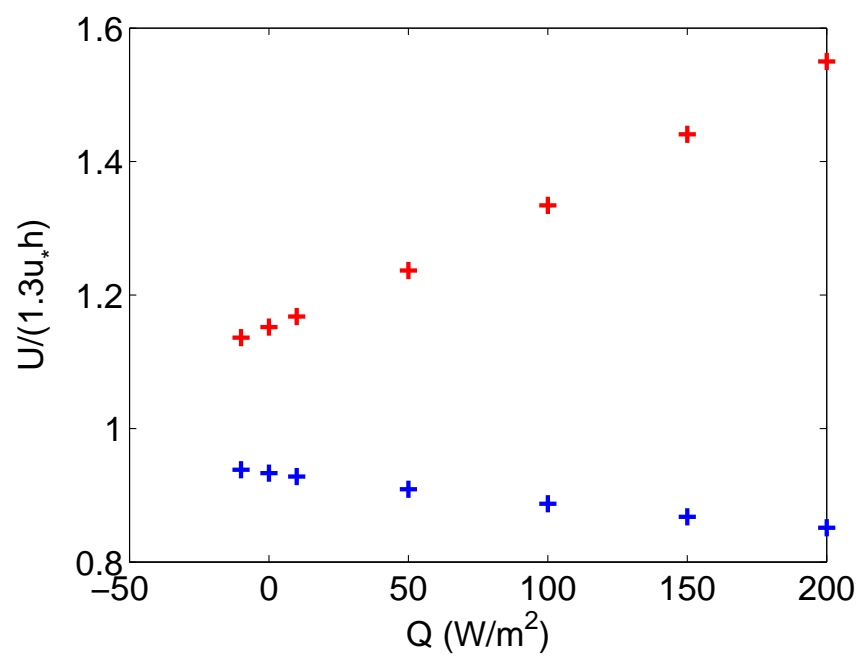

Figure 5-18: Transport fraction as a function of surface heat flux for onshore (blue) and offshore (red) wind stresses. $Q$ ranges from -10 to $200 \mathrm{~W} / \mathrm{m}^{2}$ for $\tau^{x}=0.1 \mathrm{~Pa}$ at $10 \mathrm{~m}$ water depth. 


\section{Stratification as a function of $Q$}

Without wind stress, surface heat flux creates stratification by warming the near surface water. Under wind forcing, it is by the combined increase in cross-shelf density gradient and increase or decrease in transport, that a surface heat flux alters the small final stratification maintained on the inner shelf. For offshore wind stress, the surface heat flux increases the cross-shelf density gradient and increases the crossshelf transport fraction. These two effects combine to increase the final stratification on the inner shelf, which in turn feeds back positively on the transport. For onshore wind, the surface heat flux increases the cross-shelf density gradient but decreases the cross-shelf transport fraction. An increased cross-shelf density gradient for a fixed transport would be expected to make a more negative stratification by onshore wind stress. A decreased transport for a fixed cross shelf density gradient would be expected to reduce the magnitude of the negative stratification. These two tendencies have opposite effects on the stratification so it is only from the model results that we find that the small negative stratification continues to decrease (become more negative) for increasing surface heat flux.

Figure 5-19 shows the trend of increasing stratification by offshore wind stress and decreasing stratification by onshore wind stress for increasing surface heat flux values. These trends mimic those observed in the transport fraction as a function of surface heat flux, as seen in Figure 5-18.

\section{Transport scaled by $R i_{x}$}

Now that we have seen the individual relationships between surface heat flux and cross-shelf density gradient, transport, and final stratification, we reconsider the horizontal Richardson number as a scale for the transport fraction. As in section 5.4.2, we find that the data, which spread with wind stress magnitude and water depth in previous plots in this section, fall together when their effects on transport are combined into the horizontal Richardson number. Figure 5-20 shows transport fraction as a function of the horizontal Richardson number times the sign of the wind 


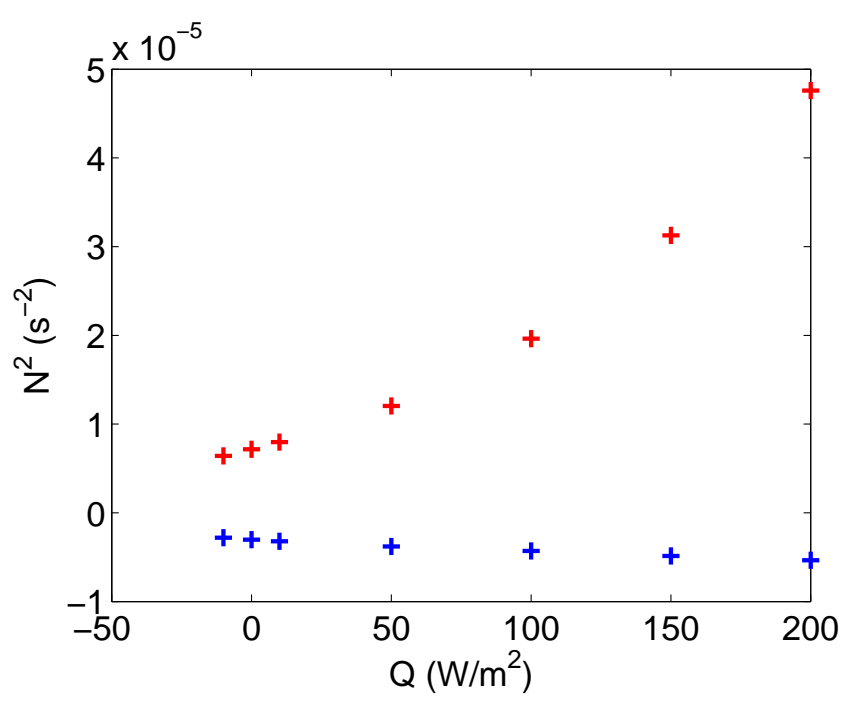

Figure 5-19: Final stratification as a function of surface heat flux for onshore (blue) and offshore (red) wind stresses. $Q$ ranges from -10 to $200 \mathrm{~W} / \mathrm{m}^{2}$ for $\tau^{x}=0.1 \mathrm{~Pa}$ at $10 \mathrm{~m}$ water depth.

stress. In 5-20(a) the density gradient used to make $R i_{x}$ is from the model output, while in 5 -20(b) the density gradient used to make $R i_{x}$ was predicted by equation $5.14 \mathrm{~d}$ from the model inputs. Transport increases more rapidly for positive values

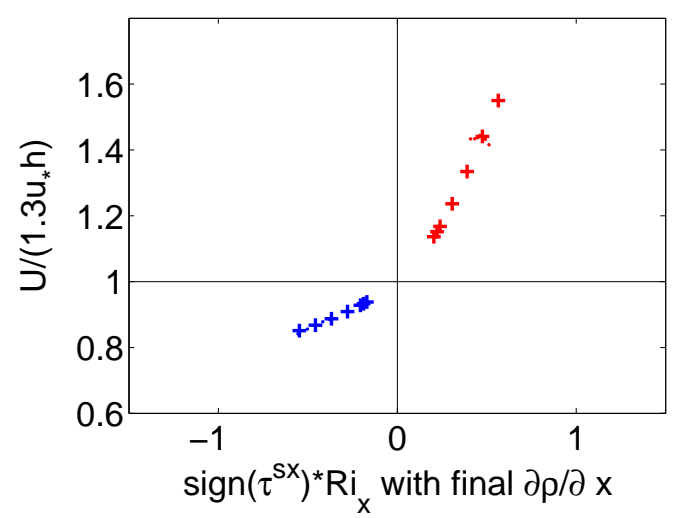

(a) Using model $\partial \rho / \partial x$

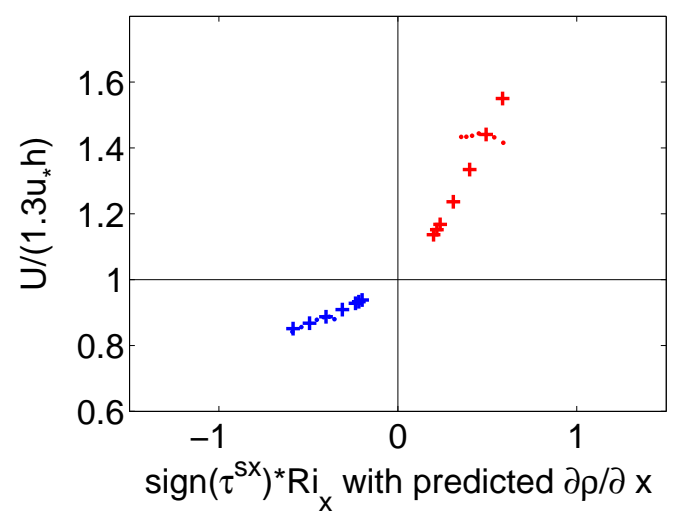

(b) Using $\partial \rho / \partial x$ predicted by eq. 5.14

Figure 5-20: Transport fraction compared to the sign of the wind stress times the horizontal Richardson number, $R i_{x}$ for modeled transport at $10 \mathrm{~m}$ water depth for surface heat flux values from -10 to $200 \mathrm{~W} / \mathrm{m}^{2}$ and for water depths from $5-12 \mathrm{~m}(\cdot)$ for $Q=150 \mathrm{~W} / \mathrm{m}^{2}$. (a) uses the cross-shelf density gradient generated by the model and (b) uses the density gradient predicted by equation 5.14

of $\operatorname{sign}\left(\tau^{x}\right) * R i_{x}$ than it decreases for negative values. This difference makes sense 
when we consider the meaning of a wind stress and a density gradient in the same direction or in opposite directions. Wind blowing from warm water over cooler water will increase stratification and transport and impede the mixing process. Wind blowing cold water over warmer water causes negative stratification near the surface first, making the water column unstable in same part of the water column where the shear in the wind-driven circulation is highest. This instability increases mixing, which will reduce shear and transport. Both the mixing and the reduced transport of buoyancy will reduce stratification, so the transport reduction will rapidly limit itself.

In Figure 5-20(b), the $\partial \rho / \partial x$ predictions for offshore (red) wind stress over varying water depths $(\cdot)$ do not lie on a single line as well as those for onshore (blue) wind stress or those in Figure 5-20(a), which used the actual $\partial \rho / \partial x$ values generates by the model. The difference is due to the prediction's underestimation of $\partial \rho / \partial x$ very near the coast and overestimation of $\partial \rho / \partial x$ towards the outer edge of the inner shelf for upwelling wind stresses. That difference can be observed in Figure 5-16(a), where the prediction (black) line is below the modeled (red) line for depths up to $8 \mathrm{~m}$ and above it for $9 \mathrm{~m}$ and deeper.

\subsubsection{Deep water boundary layer thickness}

In deep water, the surface heat flux warms the entire surface mixed layer, increasing the density jump across the base of the mixed layer. Since deep water boundary layer thickness is in part limited by the strength of the stratification below it, we check that the deep water boundary layer thickness is not strongly influencing the results from this section that we have attributed to the inner shelf cross-shelf density gradient. In our model, the deep water boundary layer develops to a $16 \mathrm{~m}$ thickness for runs with 0.1 Pa wind stress and surface heat flux ranging from -10 to $100 \mathrm{~W} / \mathrm{m}^{2}$. The boundary layer is one grid cell, or about $2 \mathrm{~m}$, thinner for the two runs with highest surface heat flux. In contrast, for the runs with wind stress one half the size, $0.05 \mathrm{~Pa}$, the deep water boundary layer thicknesses are 10 and $8 \mathrm{~m}$ for 50 and $150 \mathrm{~W} / \mathrm{m}^{2}$ surface heat fluxes, respectively. The difference in deep water boundary 
layer thickness between simulations with varying surface heat fluxes is much smaller than change with wind stress magnitude over the range of parameters used here. As a result, this small change is boundary layer thickness with $Q$ is unlikely to substantially effect the results presented in Figures 5-20 or 5-16(a).

\subsection{Combined cross- and along-shelf wind forcing}

Until now, we have considered only the effect of purely cross-shelf wind stress. However, in the field, the wind stress is rarely purely cross-shore or along-shore. At the MVCO field site off the southern coast of Martha's Vineyard, the coastline runs nearly east-west and summertime wind stresses are typically towards the northeast, with a secondary tendency towards the southwest (Figure 5-21). It is convenient to think of

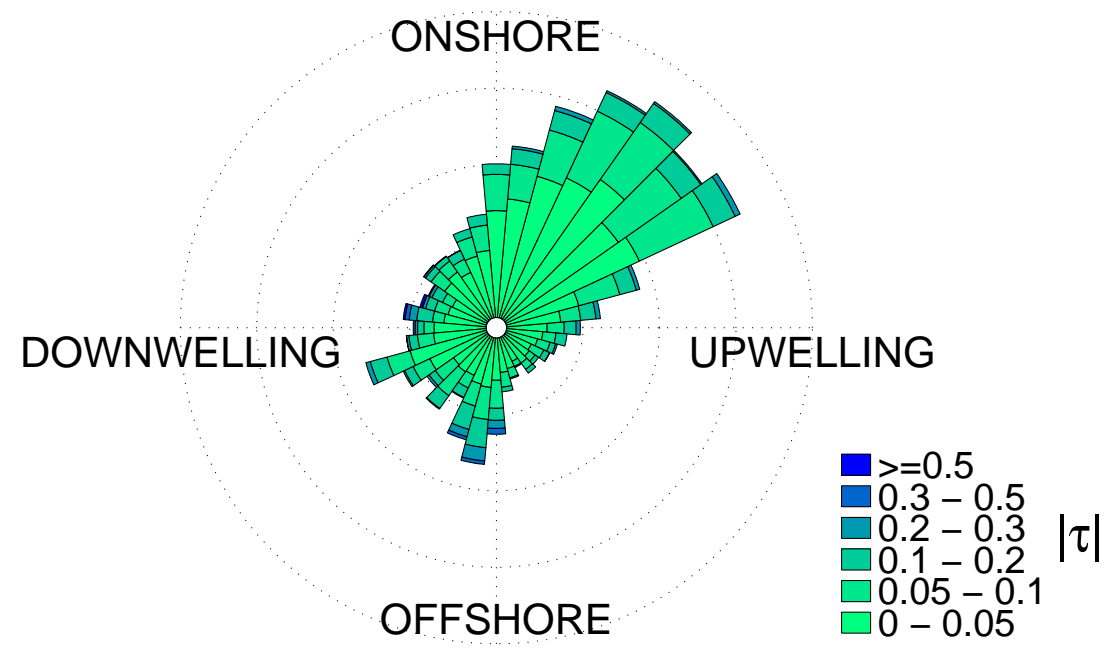

Figure 5-21: Summertime (Apr.-Oct.) wind stress from the Air Sea Interaction Tower (ASIT) at the Martha's Vineyard Coastal Observatory during the SWWIM deployments. Total bar length for each $10^{\circ}$ wedge represents percent of time wind stress is in that direction. Dashed circles are 2, 4, 6, $8 \%$ of data. Color bars withing each slice show distribution of wind stress magnitudes $(\mathrm{Pa})$ in that direction.

the response to combined along- and cross-shelf wind stresses as the sum of the circulations driven by each of the two wind stress components separately. This section was 
motivated by the particular question of whether, in light of the likely nonlinear contributions to mixing and buoyancy advection by the two components of wind stress, the cross-shelf circulation's responses to cross- and along-shelf wind stresses could reasonably be summed, as was necessarily assumed in a linear regression analysis of field data in the previous observational chapter. This experiment was performed with the intention of describing the influence of an along-shelf wind stress component on the cross-shelf circulation's response to cross-shelf wind stress. To investigate the response to combined wind forcing we use the base case model configuration (initial $\mathrm{N}^{2}=4.25 \times 10^{-4} \mathrm{~s}^{-2}$ ) and vary the wind direction from 0 to $360^{\circ}$ in $15^{\circ}$ increments while holding the wind stress magnitude constant at $0.1 \mathrm{~Pa}$. We ask, is the response to cross-shelf wind stress symmetric in the presence of an along-shelf wind stress and can a combined $\tau^{x}+\tau^{y}$ wind stress create a stratified inner shelf? And then, how does the upwelling or downwelling density front evolve, where is the outer edge of the inner shelf, and are these two transitions at the same location?

\subsubsection{Asymmetric response to $\tau^{x}+\tau^{y}$ combined wind forcing}

In the previous sections of this chapter, we have observed that a cross-shelf wind alone over an initially stratified shelf does not create a strongly stratified inner shelf. Even with the addition of a surface heat flux or an imposed cross-shelf density gradient, stratification that would be measurable in the field does not develop except under a very high surface heat flux or very strong cross-shelf density gradient combined with low wind stress. Circumstances such as these are unlikely to persist on the Martha's Vineyard shelf for extended periods of time. Models of along-shelf wind over a continuously stratified shelf (Allen et al., 1995; Austin and Lentz, 2002; Wijesekera et al., 2003) have demonstrated upwelling wind stress creates stratification over much of the shelf while Tilburg (2003) showed that for the same stratification and wind stresses used here, the $5 \mathrm{~m}$ water depth location remained unstratified for all wind directions.

With combined cross- and along-shelf wind stresses, we observe that the strongly 
asymmetric response to along-shelf wind forcing extends to the inner shelf and creates an additional asymmetry in the response to the cross-shelf wind. Figure 5-22 shows the cross-shelf transport (top row, blue dots) and stratification (bottom row) compared to the cross- (black line) and along-shelf (blue line) wind stresses at locations in the model with water depths of 7 (5-22(b)), 12 (5-22(c)), and $17 \mathrm{~m}$ (5-22(d)). These depths were chosen to match the locations of field observations discussed in Chapter 4 .
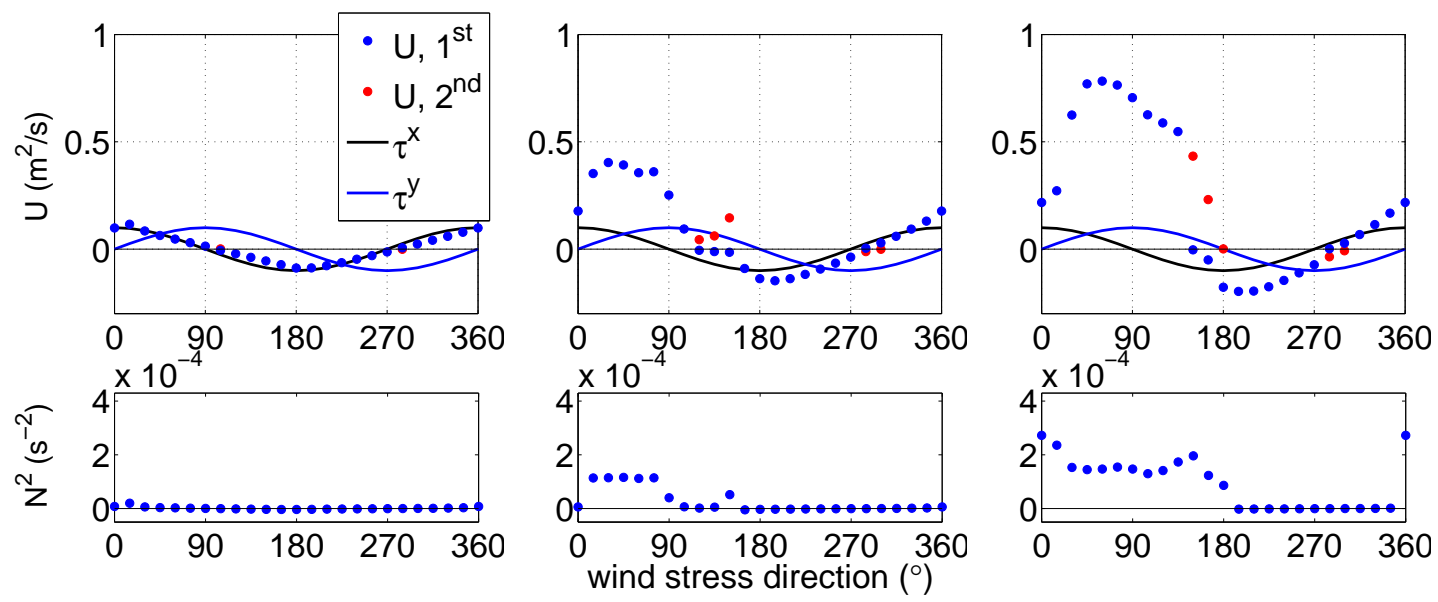

(b) $7 \mathrm{~m}$

(c) $12 \mathrm{~m}$

(d) $17 \mathrm{~m}$

Figure 5-22: Cross-shelf transport $\left(\mathrm{m}^{2} / \mathrm{s}\right)$ calculated above the first (blue dots) and second (red dots) zero crossing of the cross-shelf velocity profile, cross-shelf (black line) and along-shelf (blue line) wind stress components $(\mathrm{Pa})$ in the top row, and final stratification, $N^{2}\left(\mathrm{~s}^{-2}\right)$ in bottom row, as a function of wind direction, $0^{\circ}=$ offshore, $90^{\circ}=$ upwelling, at 7 (left), 12 (center), and $27 \mathrm{~m}$ (right) water depth

The wind stress magnitude for all model runs in this experiment is $0.1 \mathrm{~Pa}$, with $f=10^{-4} \mathrm{~s}^{-1}$ and $\rho_{0}=1025 \mathrm{~kg} / \mathrm{m}^{3}$, so full Ekman transport to the right of the wind stress is $0.98 \mathrm{~m}^{2} / \mathrm{s}$. For a nearly unstratified inner shelf, transport is expected to scale like $u_{*}^{x} h$, where $u_{*}^{x}=\sqrt{\tau^{s x} / \rho}$, as discussed in section 5.3. Indeed, at $7 \mathrm{~m}$ water depth, the transport closely follows the variation in the cross-shelf wind stress and the water column remains nearly unstratified for all wind directions. This result is consistent with Tilburg's results from $5 \mathrm{~m}$ water depth.

At $12 \mathrm{~m}$, asymmetry develops in both the transport and the stratification. For 


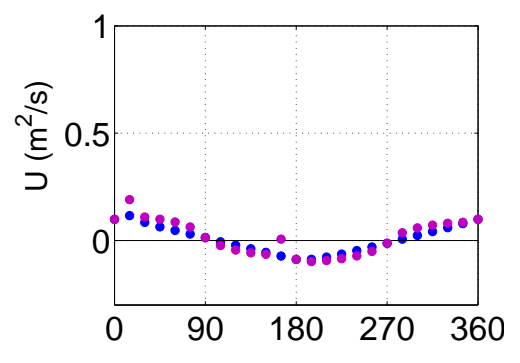

(b) $7 \mathrm{~m}$

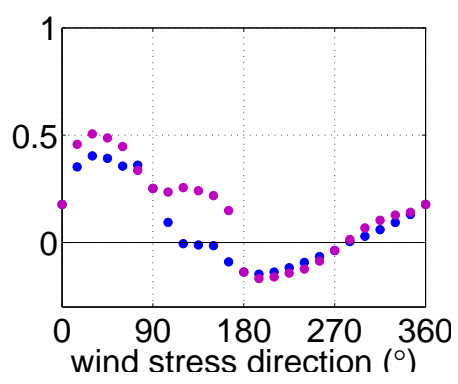

(c) $12 \mathrm{~m}$

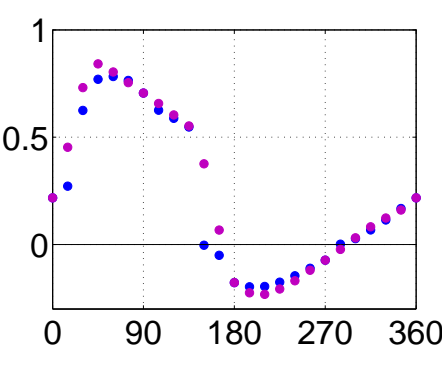

(d) $17 \mathrm{~m}$

Figure 5-23: Cross-shelf transport $\left(\mathrm{m}^{2} / \mathrm{s}\right)$ driven by wind stress in direction indicated on horizontal axis (blue) and the sum of the transports driven by the cross- and along-shelf wind components separately (purple). $0^{\circ}=$ offshore, $90^{\circ}=$ upwelling

downwelling wind stresses $\left(180^{\circ}<\theta<360^{\circ}\right)$, the 12 -m location remains unstratified and the transport follows the shape of the cross-shelf wind stress. For upwelling wind stresses, the transport is elevated for the $0-90^{\circ}$ range for offshore and upwelling wind, and the water column is stratified. These features are asymmetric to the response to onshore and downwelling wind.

At $17 \mathrm{~m}$, the full range of downwelling wind stresses $\left(180^{\circ}<\theta<360^{\circ}\right)$ still creates an unstratified water column and transport following the variation in the cross-shelf wind stress. At this depth, the water column becomes stratified for the range of upwelling wind stresses $\left(0^{\circ}<\theta<180^{\circ}\right)$. The transport is higher for upwelling than downwelling wind stresses for all corresponding cross-shelf wind stress components. At $90^{\circ}, 70 \%$ of full Ekman transport is realized. However, this transport is further modified by the cross-shelf wind stress. The transport peaks at $60^{\circ}$ (offshore of pure upwelling) and decreases from 60 to $180^{\circ}$. At midshelf (not shown here), the water column will be stratified for all wind directions and transport will respond with $180^{\circ}$ rotational symmetry.

In Figure 5-23, the transports show in Figure 5-22 (blue) are compared to the sum (purple) of transports driven by each wind stress component separately. At the 12-m location (5-23(c)) the difference is noticeable for all upwelling wind stresses, and largest in the upwelling/onshore range $\left(90^{\circ}<\theta<180^{\circ}\right)$. At $17 \mathrm{~m} \operatorname{depth}(5-23(\mathrm{~d}))$, the 
difference is most dramatic for wind stresses that have a large cross-shelf component combined with a small upwelling component $\left(15,30,150,165^{\circ}\right)$. The individual contributions from along- and cross-shelf wind stresses to the summed transport are show in Appendix 5.A. It is notable that at $12 \mathrm{~m}$ on a stratified inner shelf, the transport does not monotonically increase for increasing magnitudes of upwelling wind stress. Blue dots in Figure 5-30(c) show a decreasing transport from 60 to $90^{\circ}$ (and 120 down to $90^{\circ}$ ), for increasing upwelling wind stress. The stronger upwelling wind stress creates more stratification by stronger advection, but also creates more mixing, which destroys it.

In Figures 5-22(b) 5-22(c), at $120^{\circ}$ and $150^{\circ}$ respectively, we note the abrupt jump in transport magnitude and sign. These angles are both between upwelling and onshore wind stress. The jump is a result of our choice to calculated transport by integrating to the first zero crossing of the cross-shelf velocity profile for all wind angles and water depths. At these wind angles, the upwelling component of wind stress drives offshore transport in the upper portion of the water column and the onshore component opposes this circulation, forcing the very near surface velocities back to a negative value. Figure 5-24 shows cross-shelf velocity profiles from the 12-m location for every $30^{\circ}$ in the range of upwelling wind stress directions. At this depth, profiles from 120 to $150^{\circ}$ have two zero crossings.

For some transport applications, it may make sense to integrate to the second zero crossing of the cross-shelf velocity profile instead. Where the cross-shelf velocity profile has more than one zero crossing, transport calculated to the second zero crossing is also indicated in Figure 5-22 (red dots). However, at mid-shelf, any direction wind stress drives a full Ekman spiral in the surface boundary layer. For a cross-shelf wind, the velocity profile will have two zero crossings, at the middle and bottom of the surface boundary layer, and for an along-shelf wind, the velocity profile can have up to four, two each near the tail ends of both boundary layers. This example highlights the sensitivity of the transport calculation to the application of transport we are interested in. Here, we choose to integrate to the first zero crossing of the 
velocity profile for all wind directions and water depths while keeping in mind that when the two wind stress components would independently drive opposite direction surface velocities, the abrupt transition in our transport calculation occurs at one point in a gradual transition in the model dynamics. We also note the local peak in stratification at $150^{\circ}$ associated with the jumps in transport at the 12 and $17 \mathrm{~m}$ depths; this feature remains unexplained.

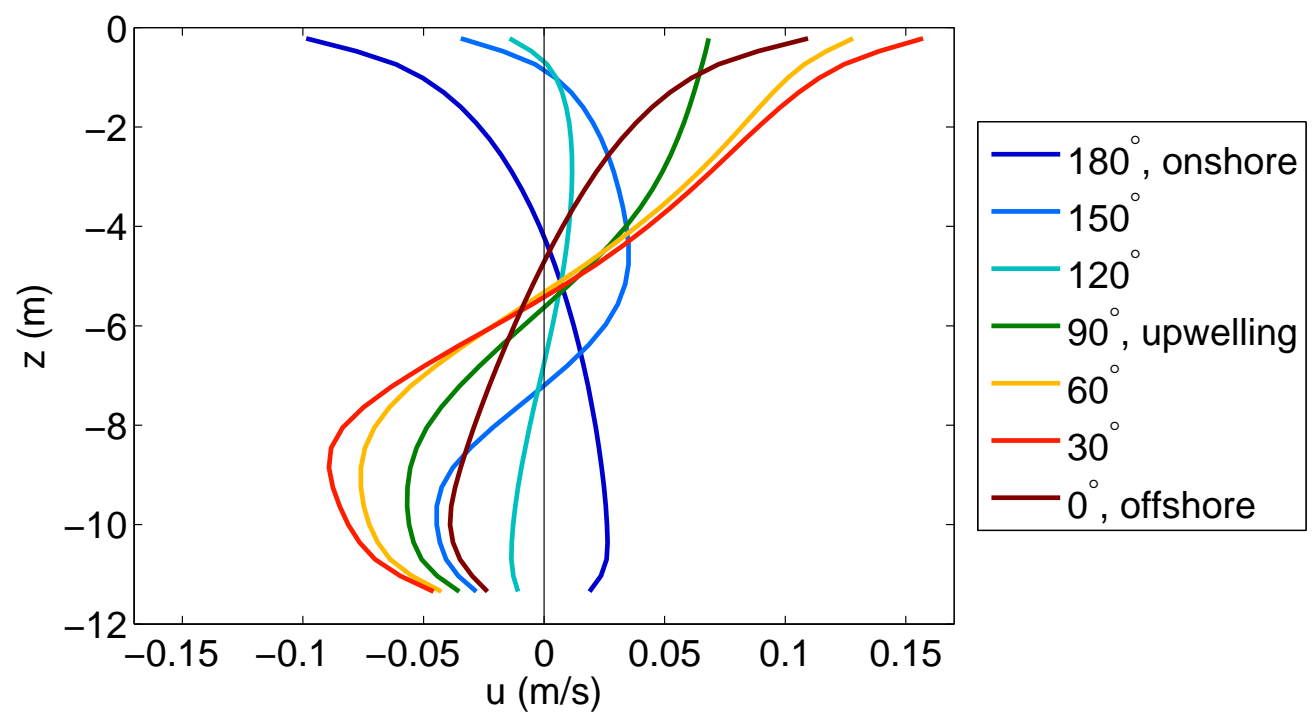

Figure 5-24: Profiles of cross-shelf velocity $(\mathrm{m} / \mathrm{s})$ in $12 \mathrm{~m}$ water depth driven by 0.1 $\mathrm{Pa}$ wind stress at upwelling wind directions varying from 0 to $180^{\circ}$ in $15^{\circ}$ increments

\subsubsection{Example of equal parts $\tau^{x}$ and $\tau^{y}$ wind stress}

An upwelling wind stress drives an offshore cross-shelf component to the surface velocity so the addition of an onshore wind stress opposes that circulation, while an offshore wind stress enhances it. Cross-shelf transects of density, cross-shelf velocity, and along-shelf velocity from two model runs with wind stresses at $45^{\circ}$ onshore and offshore of upwelling show the differences in the inner shelf response to upwelling/onshore and upwelling/offshore configurations. Figure 5-25 shows density, cross-shelf velocity, and along-shelf velocity transects for purely upwelling wind stress (middle row) compared to those for $45^{\circ}$ onshore (top row) and $45^{\circ}$ offshore (bottom 
row) of upwelling. This discussion focuses on the cross-shelf circulation in 5-25(b), but we also note that the along-shelf velocity in 5-25(c) shows that while both circulations develop a double jet structure, the along-shelf flow is much stronger for the onshore cross-shelf wind component and the density structure in 5-25(a) at the same location shows isopycnals tilted towards the coast creating stronger stratification in the upper portion of the water column for the onshore wind case.

In Figure 5-25(b), the maximum cross-shelf velocities driven by the upwelling/offshore wind are larger and the cross-shelf transport is confined closer to the surface than for the upwelling/onshore case. With an offshore wind component (bottom row), the circulation is continuous across the inner shelf, with streamlines entering the domain at the deep boundary, passing through the shallowest few kilometers near the coast, and upwelling at a range of locations. With an onshore wind component (top row), streamlines entering the domain at the deep boundary all upwell near $10 \mathrm{~km}$ offshore and block transport to the shallowest part of the domain. A separate, closed, streamline indicates opposite direction circulation (onshore at near the surface) onshore of $10 \mathrm{~km}$. These results from initially stratified model runs resemble the analytic model solution for the unstratified case of combined along- and cross-shelf wind forcing presented by Estrade et al. (2008)

For purely upwelling wind stresses over an initially stratified shelf, the slope Burger number, $S \equiv \alpha N / f$, determines if the return flow is confined to a bottom boundary layer $(S<1)$ or distributed throughout the stratified interior $(S>1)$ (Lentz and Chapman, 2004). For larger Burger numbers and an interior return flow, Austin's (1998) numerical model showed upwelling circulation and advected stratification penetrating across the inner shelf to the coast. For small Burger numbers with a bottom boundary layer return flow, isopycnals upwelled and outcropped offshore of the coastal boundary, pinching off and isolating a region of lighter water near the coast. For all model runs in this section, $S=0.21<1$, so a warm inner shelf, isolated from the midshelf by upwelling velocities and outcropping isopycnals, is expected. Indeed, our purely upwelling wind stress run (middle row of Figure 5-25) does exhibit the 
predicted behavior. Streamlines rise from the bottom boundary layer to the surface around $10 \mathrm{~km}$ from shore and no cross-shelf circulation develops onshore of the upwelling. Results shown in Figure 5-25(b), demonstrate that the cross-shelf wind component can alter the applicability of the Burger number criteria to determine the connectivity of streamlines across an upwelling inner shelf.
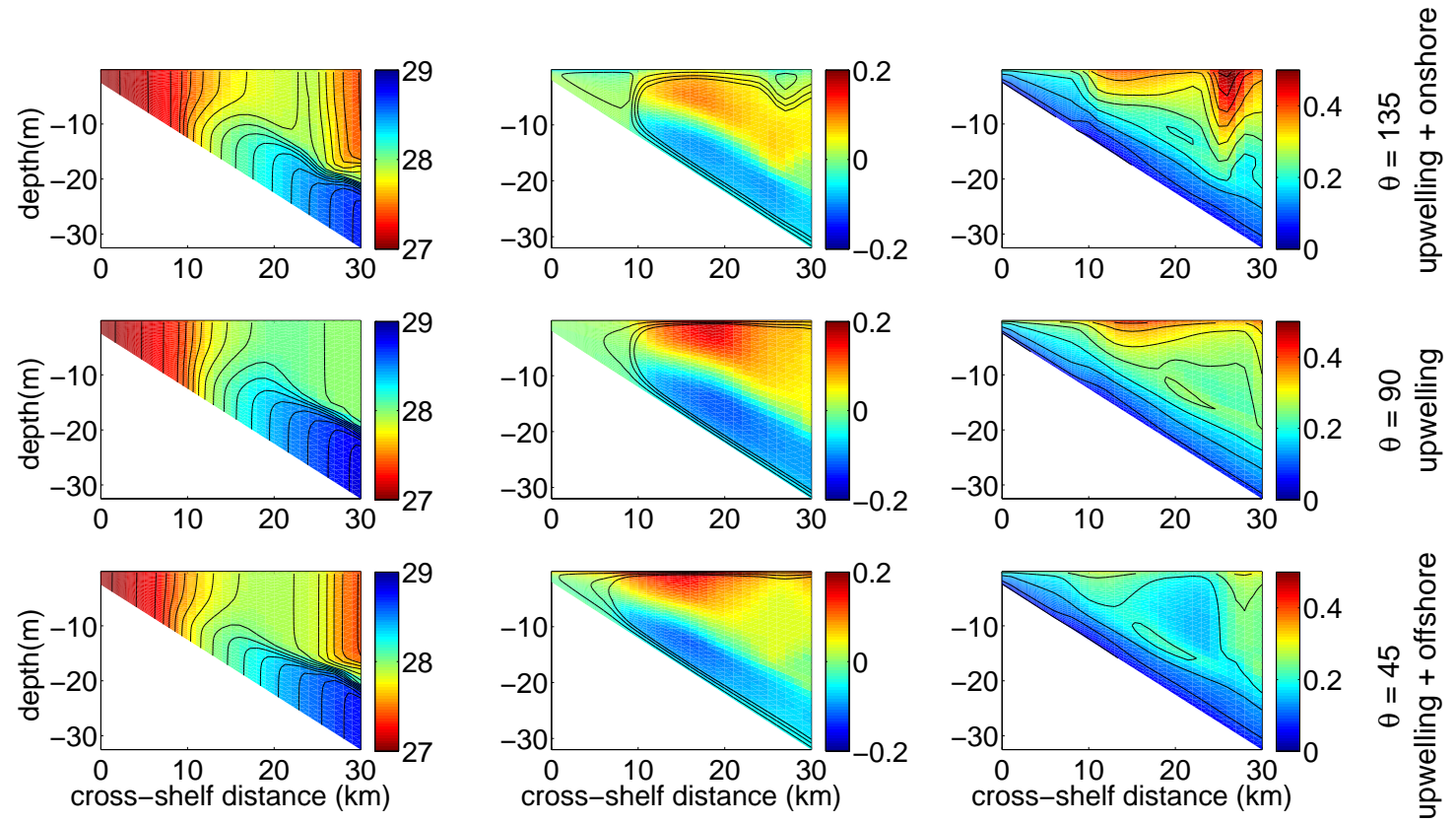

(a) $\rho\left(\mathrm{kg} / \mathrm{m}^{3}\right)$

(b) $u(\mathrm{~m} / \mathrm{s})$ and $\psi / \frac{\tau^{x}}{\rho f}$

(c) $v(\mathrm{~m} / \mathrm{s})$

Figure 5-25: Inner shelf fields of (a) density color and contours, (b) cross-shelf velocity color and streamfunction contours, and (c) along-shelf velocity color and contours. Contour intervals are $0.1 \mathrm{~kg} / \mathrm{m}^{3}, 0.04,0.05 \mathrm{~m} / \mathrm{s}$ for (a), (b), and (c). The circulations were driven by $135^{\circ}=$ upwelling + onshore (top row), $90^{\circ}=$ upwelling (middle row), and $45^{\circ}=$ upwelling + offshore (bottom row).

\subsubsection{Defining the inner shelf}

In Figure 5-22, we saw the asymmetric and continuous transition from the cross-shelf wind stress dominated transport regime of the inner shelf, to the along-shelf wind stress dominated transport of the mid-shelf. Before we continue with the discussion of the effect of stratification on the inner shelf, we must pause to define what inner 
shelf means in this context. The term inner shelf has been used in a variety of ways in the past, alway beginning outside of the surfzone, its extent is usually defined as the location where the upper and lower boundary layers overlap and there is divergence in the Ekman transport (Lentz, 1995) or as the region onshore of the surface density front that is created by up- or downwelling wind stresses (Austin and Lentz, 2002).

In this model, there is no beach or surfzone, only a gradually sloping bottom up to a shallow vertical wall. We have observed in Figure 5-22(c) that both cross- and along-shelf wind stresses are important in driving cross-shelf transport in the stratified region. For model runs with both cross- and along-shelf wind stress components, we use a Richardson number $\left(R i=(\partial u / \partial z)^{2} / N^{2}\right)$ criteria to determine when the surface boundary layer is separate from the bottom boundary layer, or from the bottom if there is no bottom boundary layer. Here, the inner shelf is the region onshore of the first location where somewhere in the water column, $R i>0.5$. The turbulence closure scheme in the model has a nominal "critical" Richardson number of 0.21 for suppression of turbulence. However, the functional form of the eddy viscosity has a steep but smooth decrease for Richardson numbers greater than 0.21 and we find the slightly higher value provides a better measure of the extent of the inner shelf. In particular, the purely cross-shelf wind stress velocity profiles have a zero-shear point at about $10 \%$ of the water depth above the bed (see $z \sim-11 \mathrm{~m}$ on 0 and $180^{\circ}$ profiles in Figure 5-24) with $R i$ values between 0.2 and 0.5 at that height across the inner shelf. For $R i=0.5$, our model output has the eddy viscosities around $10^{-3} \mathrm{~m}^{2} / \mathrm{s}$, which is two orders of magnitude higher than the model background value. Where the boundary layer separates from the bottom, the Richardson number is well over 1. For model runs with an along-shelf wind component, defining the inner shelf by maximum $R i$ values between 0.21 and 1 have qualitatively similar results.

In Figure 5-26, density and cross-shelf velocity transects are shown for the same onshore/upwelling wind case as in the top row of Figure 5-25. In Figure 5-26, the central of the three vertical lines in each panel indicates the location of the first profile with an $R i>0.5$ value. The left vertical line in each panel indicates the location 
of maximum depth-averaged vertical velocity. The right vertical line indicates the location of the surface density front.

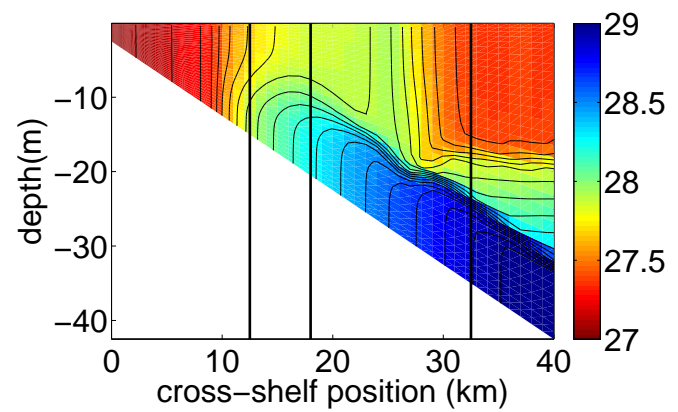

(a) Density anomaly $\left(\mathrm{kg} / \mathrm{m}^{3}\right)$

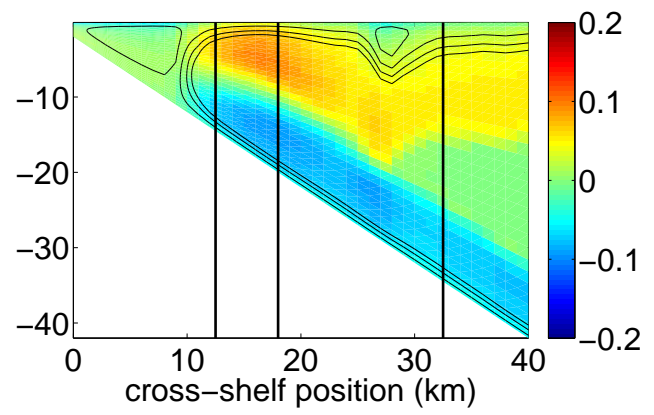

(b) Cross-shelf velocity $(\mathrm{m} / \mathrm{s})$ and streamfunction $\psi / \frac{\tau^{x}}{\rho f}$

Figure 5-26: Density anomaly (a) and cross-shelf velocity (b) for onshore + upwelling wind stress. Vertical lines indicate, from left to right, the locations of maximum depth-averaged vertical velocity, the first $R i>0.5$, and the surface density front.

\subsubsection{Stratification and transport for all wind directions and water depths}

Here we extend our view of transport (5-27(b)) and stratification (5-27(a)) to include all wind directions. In the polar plots of Figure 5-27, distance from the center represents water depth in meters, and angle is wind stress direction, with $0^{\circ}$ offshore and $90^{\circ}$ upwelling. The superimposed lines, which are the same on both plots, indicate the locations of the surface density front (dashed) and the first depth that has $R i>0.5$ (solid). All values are from the end of the 5-day model runs.

For downwelling wind stresses $\left(180^{\circ}<\theta<360^{\circ}\right)$, the surface density front, the outer edge of the inner shelf $(R i>0.5)$ and sharp increases in stratification and transport all occur within a few kilometers of each other, spanning 35 to $40 \mathrm{~m}$ water depth across the width of the downwelling front. The negative vertical velocity (not shown) associated with downwelling is also limited to a region just a few kilometers wide. Offshore of the downwelling front, there is stratification and full Ekman transport driven by the along-shelf wind stress. Onshore of the front, there is little or even 


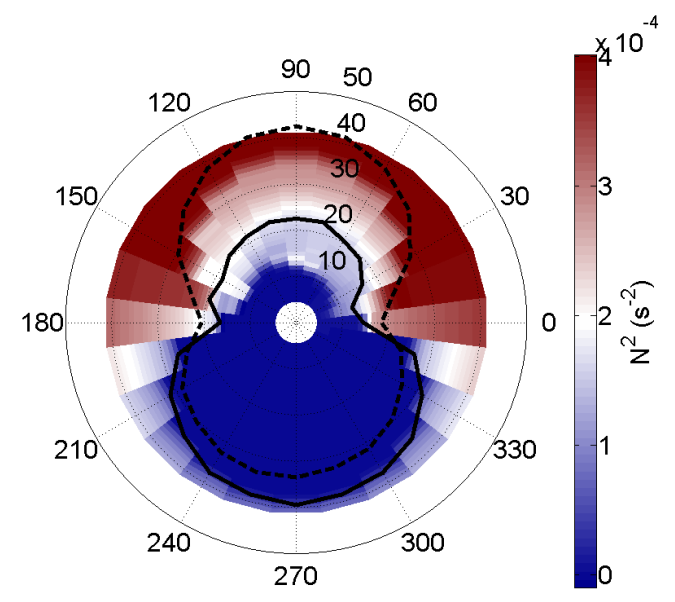

(a) Stratification

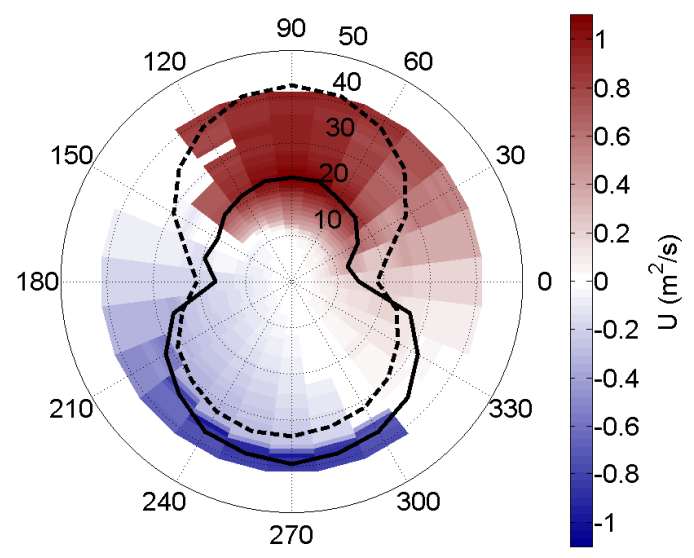

(b) Transport

Figure 5-27: Color indicates stratification (a) and cross-shelf transport, positive offshore (b) after 120 hours. Distance from the center indicates water depth (m) and compass heading indicates wind stress direction with $0^{\circ}$ offshore and $90^{\circ}$ upwelling. The dashed line indicates the location of the surface density front (density $0.02 \mathrm{~kg} / \mathrm{m}^{3}$ different from the value at the center of the model domain). The solid line indicates the first depth where some part of the water column has $R i>0.5$.

negative stratification, and transport scales like $u_{*}^{x} h$, as was described in section 5.3.

For upwelling, a dramatically different pattern emerges. The location of the $R i>0.5$ value is far onshore of the surface density front. Stratification and transport gradually increase from $u_{*}^{x} h$ at the onset of stratification to the full Ekman transport at the outer edge of the inner shelf. The transitions occur tens of kilometers apart, separated by a wide swath of vertical upwelling velocities (not shown). When upwelling winds are combined with a cross-shelf wind stress, onshore wind stress appears to reduce stratification and transport within the inner shelf by opposing the upwelling circulation, while increasing vertical mixing and so forcing the transition to midshelf dynamics to occur farther offshore, widening the inner shelf. Offshore wind stresses increase stratification and transport on the inner shelf, but also move the transition to midshelf dynamics (and reduced efficiency of the cross-shelf wind at driving transport) closer to shore. The same increased stratification that increases the cross-shelf wind driven circulation on the inner shelf also allows full separation of surface and bottom boundary layers at a shallower total water depth. 


\subsubsection{Growth of the inner shelf}

Allen et al. (1995) and Austin and Lentz (2002) described the evolution of stratification and transport by along-shelf wind stresses across the inner and mid shelves. For continuous stratification, the upwelled water eventually moves over water from a shallower (and therefore less dense) initial depth and a local increased mixing and recirculation develops, as described by Allen et al. (1995) and Wijesekera et al. (2003). Wijesekera et al. (2003) in particular noted the extended region of stratification and transitional transport between the beginning of upwelling and complete boundary layer separation. Here we provide one example of how the evolution of the inner shelf is affected by the onshore or offshore component of wind stress.

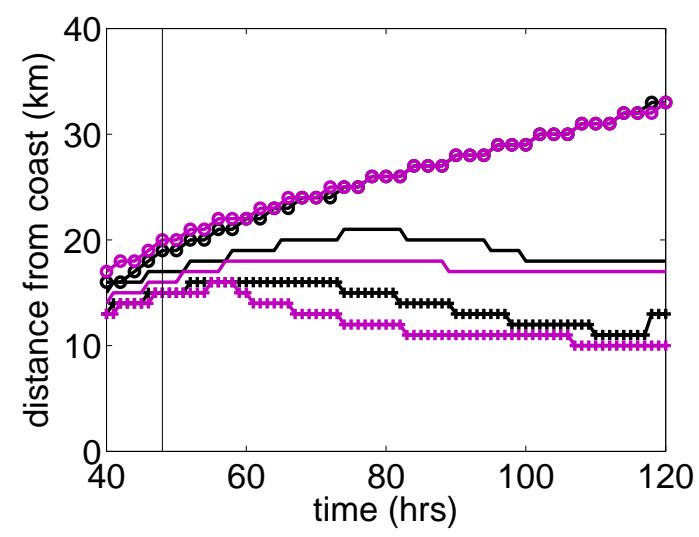

(a) Upwelling

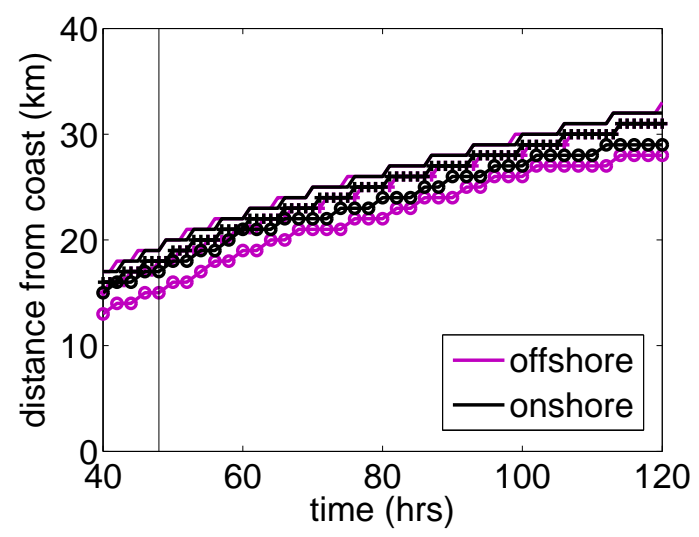

(b) Downwelling

Figure 5-28: Evolution of the position of the surface density front (o), first location with $R i>0.5$ (solid line), and maximum amplitude of the depth-averaged vertical velocity (+) for wind stresses at $45^{\circ}$ onshore (black) and offshore (blue) of upwelling (a) and downwelling (b). Vertical line indicates time at which wind stress reached full $(0.1 \mathrm{~Pa})$ amplitude after initial two-day build up.

Figure 5-28 shows time series of the cross-shelf positions of of the maximum depthaveraged vertical velocity $(+)$, the edge of the inner shelf defined by $R i>0.5(-)$, and the surface density front ( $\circ$ ) defined as a $0.02 \mathrm{~kg} / \mathrm{m}^{3}$ different from center of model domain. An example of the locations of these indices in the context of crossshelf density and velocity transects was shown in Figure 5-26. We note first that the evolution of the surface density fronts (symbol (o) in Figure 5-28) follow the 
pattern described by Austin and Lentz (2002), with the upwelling front position $\sim \tau^{s y} t$ and the downwelling front position $\sim \tau^{s y} \sqrt{t}$. For a downwelling along-shelf component (Figure 5-28(b)), the position of the maximum downward velocity $(+)$ and first $R i>0.5$ value $(-)$ are offshore of the surface density front, but their location relative to the surface front remain constant in time.

For an upwelling along-shelf component (Figure 5-28(a)), the position of the maximum downward velocity and $R i>0.5$ value do not follow the location of the surface density front. The $R i>0.5$ location progresses offshore less rapidly than the surface front, then moves back onshore before reaching a steady location. While downwelling happens over a narrow region and upwelling circulation occurs across a wide swath of inner shelf, the upwelling velocity is maximum near the onshore edge of the upwelling region. For upwelling winds, this maximum vertical velocity, which is associated with the maximum divergence in the Ekman transport, is onshore of the other two markers of the outer edge of the inner shelf and progresses towards shore possibly steadying at the end of these model runs. Throughout the model runs, both the $R i>0.5$ location and the maximum upwelling location are farther from shore for the onshore wind stress case. The onshore wind acts on the cross-shelf density gradient to destabilize the water column, slowing the penetration of upwelling circulation towards the coast. The apparent steadying of the $R i>0.5$ and maximum upwelling locations near the end of these model runs also warrants further investigation. The stabilization occurs earlier for runs with larger upwelling wind stress components, while the separation between onshore and offshore wind components is more pronounced for runs with larger cross-shelf wind stress components.

\subsection{Summary and Conclusion}

This chapter was motivated by a discrepancy between field observations and previous modeling work that left open the question: how does cross-shelf wind stress drive circulation on a stratified inner shelf? We use an idealized, 2D, cross-shelf transect 
in ROMS to describe the effects of stratification, wind stress magnitude, surface heat flux, cross-shelf density gradient, and wind direction on the inner shelf response to the cross-shelf component of the wind stress. To first order, the cross-shelf transport, $U$, scales like $u_{*} h$, but the transport fraction, $U / u_{*} h$, does vary with many environmental factors.

Over sloping bathymetry, vertical mixing of initial stratification or a surface heat flux creates a cross-shelf density gradient. Under cross-shelf wind stresses, the resulting cross-shelf gradient for water depths less than the deep water boundary layer thickness is well-represented by a prediction based on complete vertical mixing of all contributions to the density field (equation 5.15).

$$
\begin{aligned}
\frac{\partial \rho}{\partial x} & =\left(\frac{\partial \rho}{\partial x}\right)_{N^{2}}+\left(\frac{\partial \rho}{\partial x}\right)_{Q}+\left(\frac{\partial \rho}{\partial x}\right)_{0} \\
& =-\left.\frac{\alpha}{2} \frac{\partial \rho}{\partial z}\right|_{0}+\rho_{0} \alpha_{T} \frac{Q t}{\rho c_{p}}\left(\frac{\alpha}{h^{2}}\right)+\left(\frac{\partial \rho}{\partial x}\right)_{0}
\end{aligned}
$$

From modeling runs with purely cross-shelf wind stresses (sections 5.3, 5.5, 5.4), we demonstrated that advection of the cross-shelf density gradient increases transport by offshore winds and decreases transport by onshore winds, but does not generate a strongly stratified inner shelf for either wind direction. For a positive cross-shelf density gradient (lighter water near the coast), an offshore wind stress moves lighter over denser water, creating a small positive stratification, which allows increased vertical shear in the cross-shelf velocity profile and increased transport. The increased transport feeds back positively on the increased stratification until the process is limited by shear instability. An onshore wind stress moves denser over lighter water, creating a small negative stratification, which increases mixing and decreases transport. The decreased transport reduces the buoyancy advection that creates the negative stratification and the increased mixing also destroys the negative stratification, so the process that alters transport by onshore wind stress is more self-limiting than that for offshore wind stress. The relationship between cross-shelf transport and cross-shelf 
density gradient collapses for all model runs when both values are nondimensionalized with the relevant scales of the problem. The transport, $U$, is scaled by the unstratified inner shelf transport scale, $1.3 u_{*} h$, and the cross-shelf density gradient is scaled by the sign of the wind stress times the horizontal Richardson number, $R i_{x}$, $\operatorname{sign}\left(\tau^{x}\right)\left(g h^{2} / \rho u_{*}^{2}\right)(\partial \rho / \partial x)$. Figure 5-29 shows all data from Figures 5-15 and 5-20 combined.

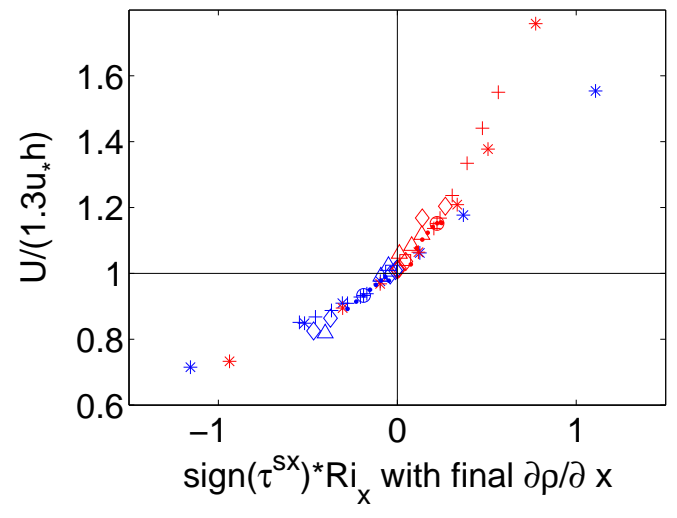

(a) Using model $\partial \rho / \partial x$

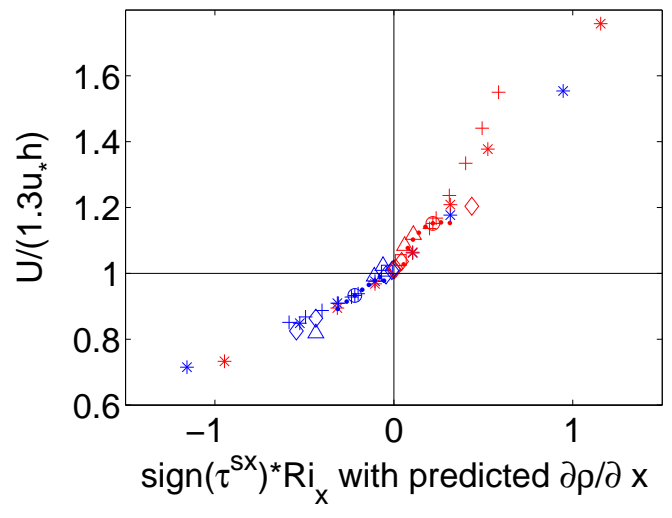

(b) Using $\partial \rho / \partial x$ predicted by eq. 5.15

Figure 5-29: Model output transport fraction, $U /\left(1.3 u_{*} h\right)$, compared to the sign of the wind stress times the horizontal Richardson number, $R i_{x}$ for (a) model final $\partial \rho / \partial x$ and (b) $\partial \rho / \partial x$ predicted by purely vertical mixing. Color indicates an onshore (blue) or offshore (red) wind stress, and the experiment variable is indicated by the symbol: base case (०), 5 - $12 \mathrm{~m}$ water depth $(\cdot)$, wind stress magnitude $(\triangle)$, initial stratification $(\diamond)$, initial cross-shelf density gradient $(*)$, and surface heat flux $(+)$.

Using model runs with wind forcing that is not purely cross-shelf (section 5.6), we find that circulation driven by cross-shelf wind forcing is strongly asymmetric when combined with an along-shelf wind stress. With a downwelling component of wind stress, the inner shelf remains unstratified and the cross-shelf wind dominates the cross-shelf circulation and transport. In contrast, an upwelling wind stress has the potential to create a strongly stratified inner shelf. On a stratified inner shelf, both along- and cross-shelf wind components contribute to the transport and affect the final stratification (Figure 5-22). The cross-shelf wind stress plays an especially large role in setting the direction and magnitude of the surface velocity (Figure 524). Since the cross-shelf wind is the dominant driver of circulation in the shallow, 
unstratified region, the cross-shelf component of wind stress can extend (offshore wind) or terminate (onshore) the reach of an upwelling circulation to connect the midshelf to near shore (Figure 5-25).

These results demonstrate that because of the importance of upwelling winds in creating stratification, it is critical to know the along-shelf wind component to predict the circulation driven by cross-shelf wind over a stratified shelf. At the same time, the cross-shelf wind component is critical in the shelf-to-shore transport because of how it enhances or opposes the nearshore extent of an upwelling circulation. Where the inner shelf becomes well mixed, it is the cross-shelf rather than vertical density gradient that is critical to predicting the transport driven by the cross-shelf wind stress. 


\section{A Appendix: Components of the combined cross- and along-shelf wind forcing}

In Figure 5-23, the purple dots are described as the sum of the transports driven by each component of the wind stress separately. These data were generated to compare to the model output for $0.1 \mathrm{~Pa}$ winds blown in directions every $15^{\circ}$ around the compass. In the top row of Figure 5-30, the individual transports are show along with their sum. In the bottom row, the final stratifications for each wind component are shown. Note that for the along-shelf components (blue), the data are symmetric around 90 and $270^{\circ}$, and for the cross-shelf components (black), the data are symmetric around 0 and $180^{\circ}$.
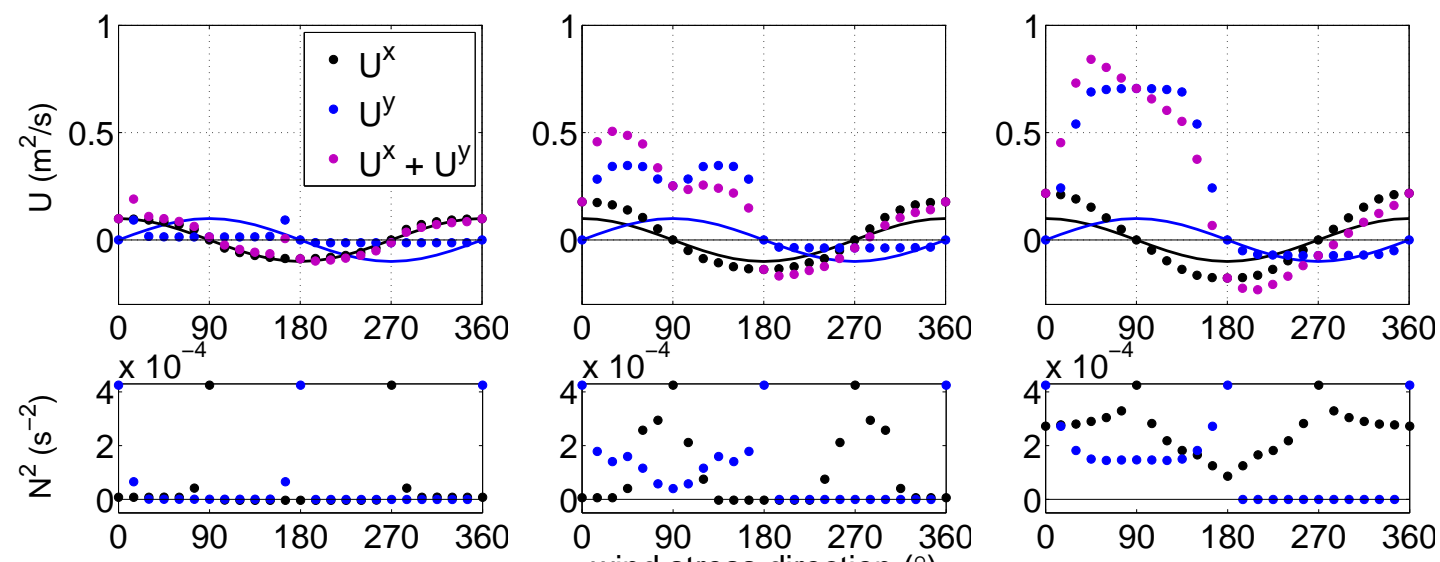

(b) $7 \mathrm{~m}$

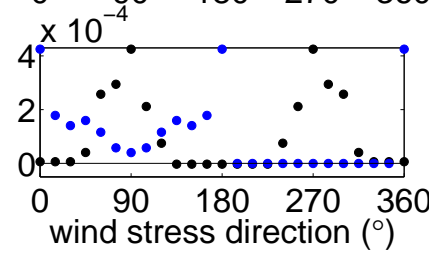

(c) $12 \mathrm{~m}$

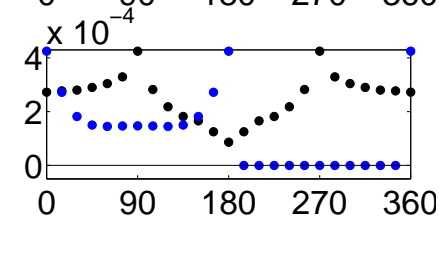

(d) $17 \mathrm{~m}$

Figure 5-30: Top row: Cross-shelf transport $\left(\mathrm{m}^{2} / \mathrm{s}\right)$ driven by the cross-shelf (black dots) and along-shelf (blue dots) components of wind stress separately, and the sum of the two transports (purple dots). Cross-shelf (black line) and along-shelf (blue line) wind stress components $(\mathrm{Pa})$. Bottom row: final stratification, $N^{2}\left(\mathrm{~s}^{-2}\right)$ after runs with only cross-shelf (black) or along-shelf (blue) wind stress. All variables show as a function of wind direction that would result from combining the two components of wind stress used separately. $0^{\circ}=$ offshore, $90^{\circ}=$ upwelling 


\section{Chapter 6}

\section{Discussion and conclusions}

The research presented in this thesis furthers the understanding of the effect of stratification on wind-driven, cross-shelf circulation. Each chapter has addressed separate aspects of the influence of stratification on the inner shelf. In this chapter, we discuss the connections between results from Chapters 3, 4, and 5. Section 6.1.1 explains why an idealized two-dimensional numerical model was best suited to address questions raised by field observations. Section 6.1.2 describes how modeling results inform our interpretation of the field observations. Following the discussion, a summary in section 6.2 highlights the key results of the thesis and section 6.3 concludes with directions for future work.

\subsection{Discussion}

\subsubsection{Choosing an idealized numerical model}

The analyses of observations in Chapters 3 and 4 are based on the assumption of along-shelf uniformity. This assumption, combined with the no flow through the coast constraint, is what tells us $\bar{u}=0$ when we consider the steady shallow water equations. The relevance of the model set up in Chapter 5, in turn, depends on the significance of two-dimensional processes at Martha's Vineyard and on other inner 
continental shelves.

The discussion in section 4.5.3 demonstrated that the wind-driven velocities at Martha's Vineyard are consistent with a two-dimensional circulation. Along the SWWIM transect, the cross-shelf circulation is dominated by the vertical structure of the wind-driven flow, with secondary but significant circulations driven by wave undertow and a depth-average (probably tidally rectified) flow. A three-dimensional model of the region by Ganju et al. (2011), run with realistic bathymetry, tides, and synoptic forcing, but no stratification, showed that the non-divergent nature of circulation across our SWWIM transect is not representative of all transects across the Martha's Vineyard inner shelf. Those model results raise the question of the usefulness of a two-dimensional view of cross-shelf transport. However, a model like that of Ganju et al. (2011) still relies on observations for verification and on preexisting theory for a dynamical explanation. For example, Weisberg et al. (2001) found that with a realistic regional scale model of the West Florida Shelf, including the stratification was required to reproduce observed circulation and its vertical structure. In addition, the model stratification needed to be maintained with appropriate buoyancy inputs and data assimilation.

Our observations during stratified conditions at Martha's Vineyard demonstrated some shortcomings of our understanding of the interaction between stratification and wind-driven circulation. A perfect model of all the natural complexities of the Martha's Vineyard shelf in stratified conditions would leave us with the same question that was raised by the field observations: which factors in the field were most important in determining the behavior of the system? Such little attention has been given to the role of cross-shelf wind at driving cross-shelf circulation, and in particular on the influence of stratification in changing that process, that we require a simpler model to answer a simpler question. The modeling chapter of this thesis does not attempt to reproduce the observations exactly, or even approximately. We have taken the questions arising from the observations and designed an experiment to test them. Using a idealized numerical configuration, we can isolate the effects of 
individual forcing mechanisms on the significant and often dominant two-dimensional features of wind-driven cross-shelf circulation.

\subsubsection{Model results inform our interpretation of field obser- vations}

We interpret the asymmetry observed between the stratified response to onshore and offshore wind stresses in Chapter 4 to be a result of the cross-shelf wind acting on a density field set by both wind components, which, in the field, are not evenly distributed around the compass. Based on model results from Chapter 5, the inner shelf stratification will be predominantly generated by upwelling winds and further modified by the cross-shelf wind stress. Near Martha's Vineyard, there is usually lighter water near the coast and denser water offshore throughout the year so buoyancy advection additionally increases or decreases the transport driven by offshore and onshore wind stresses, respectively, as described in section 5.3.2.

To directly compare observations with the model results for all direction of wind stress, we use a regression between the measured transport and the magnitude of the wind stress to estimate the efficiency of each direction of wind stress at driving crossshelf transport. This regression is a slight variation on the one given by equation 4.3 , which separated the effect of cross- and along-shelf wind stress components.

$$
\begin{aligned}
& U=A U_{E k}+C U_{w}+D \\
& \text { with } \\
& \qquad \begin{aligned}
U_{E k} & =\frac{\left|\tau^{s}\right|}{\rho_{\circ} f} \\
U_{w} & =-U_{S t}=-\frac{g H_{s i g}^{2}}{16 c_{p h}} \cos \theta_{w}
\end{aligned}
\end{aligned}
$$

Figure 6-1 shows values of the regression slope, $A$, resulting from data sets grouped by wind stress direction in $15^{\circ}$ increments for times when the deepest field sites at 27-m water depth was strongly stratified by a top to bottom density difference greater than $1 \mathrm{~kg} / \mathrm{m}^{3}$, equivalent to $\mathrm{N}^{2}=4 \times 10^{-4} \mathrm{~s}^{-2}$. This method of data processing was 
chosen to match the model initial conditions for the experiment described in section 5.6. The model transport from Figure 5-22 is replotted here scaled by the model Ekman transport value of $|\tau| / \rho_{o} f=0.97 \mathrm{~m}^{2} / \mathrm{s}$. Because stratified conditions with wind at certain directions, especially $0-90^{\circ}$, are rare (see Fig. 5-21), subsampling the filtered data at the low-pass cutoff frequency yields too few independent measurements to perform the regression analysis. Instead, all filtered data is used (20-minute spacing between observations) and the confidence intervals on these regressions are subsequently scaled up by a factor of $\sqrt{72}$, where 72 is the ratio of number data points used to the number of independent observations (one per day from 24-hour low pass filtered data).

A comparison of measured to modeled transport efficiency at $12 \mathrm{~m}$ water depth shows a remarkably good match in both directional dependence and magnitude. Like the model, the observed transport efficiency peaks between offshore and upwelling wind stress directions, drops to near zero between 90 and $180^{\circ}$, and follows the shape of the cross-shelf wind stress forcing between 180 and $360^{\circ}$. In both the model and the observations, the $12 \mathrm{~m}$ water depth is on the inner shelf. Observation of the density field at the $12 \mathrm{~m}$ site indicate stratification there is created and destroyed on synoptic time scales.

At $17 \mathrm{~m}$ water depth, the observed transport efficiency takes a similar shape to that predicted by the model with positive values over a $180^{\circ}$ range that is shifted clockwise from the $0-180^{\circ}$ range that represents an upwelling along-shelf component. However, the measured transport does not show the elevated efficiency over the upwelling range of wind stresses relative to the downwelling range as the model does. This difference may be explained by the regressions covering a range of wind stress magnitudes and stratifications. The transport at the $17 \mathrm{~m}$ site will be more sensitive than at the 12-m site to the thickness of the surface boundary layer, if it is separated from the bottom boundary layer, and how far offshore an upwelling or downwelling front is advected. In the model, transports were calculated after five days of wind stress and the surface density front reached to between 16 and 40m water depth depending on 

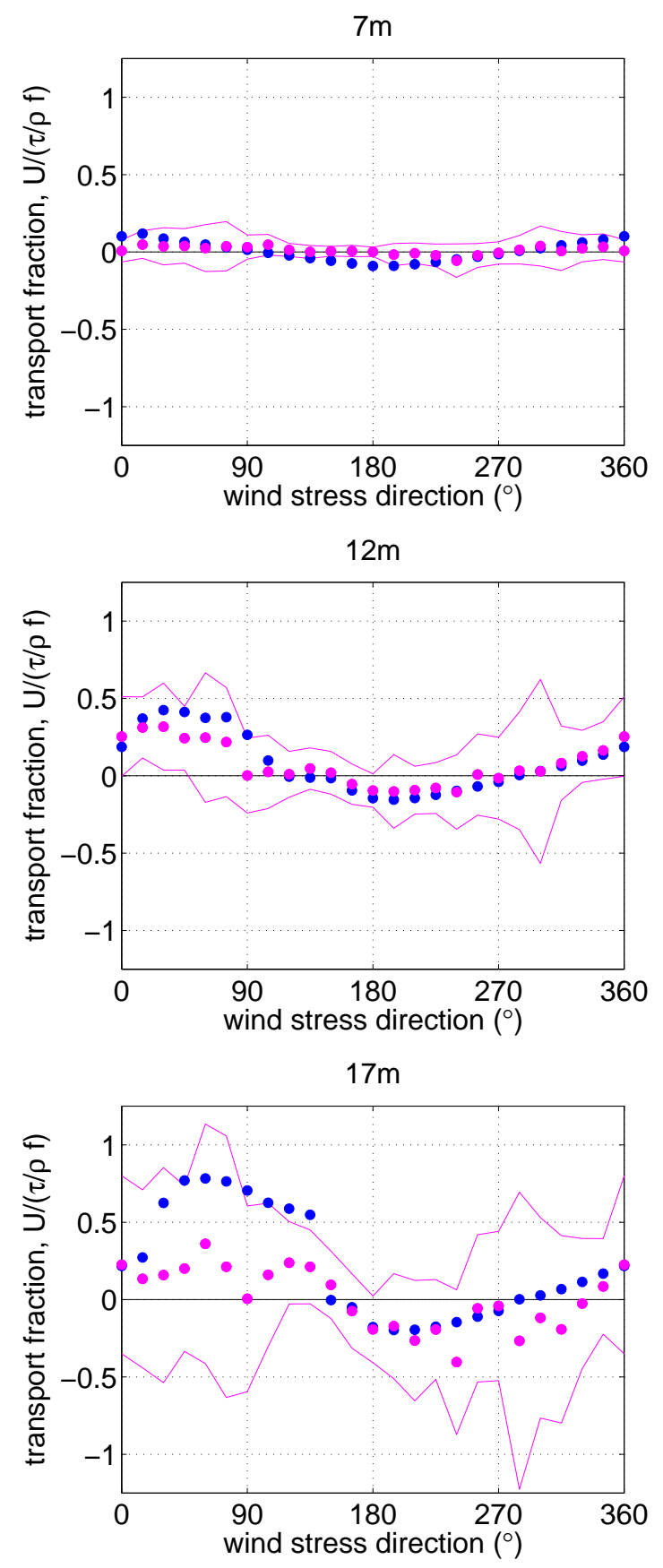

Figure 6-1: Comparison of modeled transport fraction, $U /(|\tau| / \rho f)$ (blue) with regression slope $A \sim U /(|\tau| / \rho f)$, calculated from observations (pink dots) at 7, 12, and 17 $\mathrm{m}$ water depth at Martha's Vineyard for wind in $15^{\circ}$ sections at times when the $27-\mathrm{m}$ mooring had a top to bottom density difference greater than $1 \mathrm{~kg} / \mathrm{m}^{3}$ or approx $\mathrm{N}^{2}$ $=4 \times 10^{-4} \mathrm{~s}^{-2}$, Pink lines are $95 \%$ confidence intervals on observations. $0^{\circ}$ offshore, $90^{\circ}$ upwelling. 
wind direction, making the $17 \mathrm{~m}$ location generally onshore of this front. In contrast, in the field, wind events do not typically last for five day and the density measurements suggest the $17 \mathrm{~m}$ site at MVCO is often not in the inner shelf in summer times, while the $12 \mathrm{~m}$ site is consistently on the inner shelf. The initial conditions at MVCO are also not completely quiescent, stratified, and uniform for all wind events. Since the $7 \mathrm{~m}$ site at MVCO is mixed my many summertime wind events, it is surprising that the transport there does not more closely follow the cross-shelf wind stress, as the modeled transport does. Part of the problem may be due to the relatively small velocities driven by the wind at $7 \mathrm{~m}$ depth compared to other drivers of circulation. The magnitude of modeled transport at $7 \mathrm{~m}$ ranges from 0 to $0.1 \mathrm{~m}^{2} / \mathrm{s}$ and the measured transport is even smaller. Using an average value of $0.05 \mathrm{~m}^{2} / \mathrm{s}$ distributed over the upper half of a $7 \mathrm{~m}$ water column generates a cross-shelf velocity of less than $2 \mathrm{~cm} / \mathrm{s}$.

Fewings and Lentz (2011) described a persistent upwelling circulation at the Martha's Vineyard Coastal Observatory as not wind driven due to low mean alongshelf wind stress and lack of asymmetry in the circulation's response to purely crossshelf wind stress. Our model results now suggest that a low mean along-shelf wind stress is not reason enough to discount along-shelf wind as a mechanism for creating a stratified inner shelf and that, at MVCO, upwelling winds contribute to the observed stratification. However, we note that these results do not explain the small portion of stratified conditions under downwelling wind stress observed in the field. Some mechanism other than wind-drive circulation is likely responsible for the stratification during downwelling periods and would also contribute to the stratification during all directions of wind forcing. The time-mean circulation describe in section 4.5.3 has an offshore surface flow and is one possible source of this stratification.

\section{$2 \mathrm{D}$ model of a $3 \mathrm{D}$ shelf}

The observations from the Martha's Vineyard inner shelf show a wind driven crossshelf circulation that has a vertical structure consistent with a two dimensional re- 
sponse to wind forcing. The small depth-average values of time mean and wind-driven flows (section 4.5.3) suggest along-shelf nonuniformity is not required to maintain horizontally non-divergent circulation. The match between the observed and along-shore uniform model of transport in Figure 6-1 sheds light on the dynamics driving the vertically sheared cross-shelf circulation and supports the idea that the wind-driven circulation at MVCO may indeed be predominantly two-dimensional.

The numerical model setup in Chapter 5 was designed to simulate the twodimensional response to wind forcing on the inner shelf. This idealized process study is limited in its ability to replicate the real circulation at Martha's Vineyard by the various along-shore nonuniform features as well as forces other than wind that drive circulation at this particular site. The gyre-like circulation of tidally rectified flow described by Ganju et al. (2011) is just one such example. The island alters the wind forcing around it so the wind stress is not spatially uniform and, despite the SWWIM field site being chosen in part for its relatively long, straight coastline, the seabed is streaked with rippled scour depressions in alternating bands of find and coarse sand, as described by Goff et al. (2005). The spatial variation in wind forcing and the variable bottom topography and roughness over which it acts will certain cause along-shelf non-uniformities in the circulation that are not represented in the model. Wave-driven circulation will also vary spatially, especially near the east and west ends of the island, where wave crests will refract around the sharp curves in bathymetry. As a final example, the model does not include a beach or breaking waves so does not replicate rip currents through sandbar gaps, or jets, eddies, or other transient instabilities generated in the surfzone.

\subsection{Summary}

Transport across continental shelves allows the exchange of heat, nutrients, larvae, sediment, and pollutants between coastal ecosystems and the open ocean. On the mid- and outer- shelves, along-shelf winds typically drive cross-shelf transport. On 
the inner shelf, where the surface and bottom boundary layers overlap, the alongshelf wind is ineffective at driving cross-shelf transport because momentum mixes from the surface to the bottom before the Coriolis acceleration can turn it. Recent observational (Fewings et al., 2008) and modeling (Tilburg, 2003) studies have shown cross-shelf wind stress to be a significant mechanism for cross-shelf transport on the inner shelf. Though continental shelves are typically stratified for much of the year, the inner shelf response to cross-shelf winds in stratified conditions is not well understood. This thesis uses observations from a three-year field program south of Martha's Vineyard, MA, and a numerical model to describe the effect of stratification on inner shelf circulation, transport, and sediment resuspension height. Here, we summarize the key results that have been presented in this thesis.

\subsubsection{Height of sediment resuspension}

How does stratification affect the vertical structure of the suspended sediment load?

Using acoustic backscatter intensity from an ADCP as a proxy for sediment concentration, we find that stratification just above the bottom mixed layer limits the height to which sediment is resuspended on the inner shelf (Figure 3-4). This capping effect is evident for the density gradients common near Martha's Vineyard. No large freshwater source is needed to make a sharp pycnocline. The seasonal trend of less mid water column resuspension during summer, when the water is stratified, is not just the effect of smaller waves occurring in the summer, but actually a result of the stratification.

\subsubsection{Observations of cross-shelf circulation}

How does stratification alter the inner shelf response to cross- and along-shelf wind stresses?

Stratification increases the vertical shear of the circulation and the transport driven by the cross-shelf wind stress. This effect of stratification is larger in the 
response to offshore winds than onshore winds (Figure 4-16). These observations of circulation throughout a stratified water column and the asymmetric response to onshore and offshore winds are contrary to results from a previous numerical modeling study (Tilburg, 2003) that found circulation by cross-shelf wind stress was symmetric and confined to the surface mixed layer.

\subsubsection{A model of circulation}

Which environmental factors determine the efficiency of cross-shelf wind at driving cross-shelf transport? and how does a stratified inner shelf develop?

To first order, the cross-shelf transport, $U$, scales like $u_{*} h$, but the transport fraction, $U / u_{*} h$, does vary with position on the inner shelf, $h / \delta$, and the cross-shelf density gradient. Over sloping bathymetry, vertical mixing of initial stratification or a surface heat flux creates a cross-shelf density gradient. Under cross-shelf wind stresses, advection of this cross-shelf density gradient increases transport by offshore winds and decreases transport by onshore winds, but does not generate a strongly stratified inner shelf for either wind direction. It is the cross-shelf density gradient, not the vertical one, that scales transport efficiency by cross-shelf wind stress on a mixed or weakly stratified inner shelf. The strength of the density gradient relative to the direction of the wind, nondimensionalized as a horizontal Richardson number, scales the transport efficiency for all sources of cross-shelf density gradient (Figure $5-29)$.

Using model runs with wind forcing that is not purely cross-shelf, we find that circulation driven by cross-shelf wind forcing is strongly asymmetric when combined with an along-shelf wind stress (Figure 5-22). In these model realizations, an upwelling along-shelf component was required to create stratification. On a stratified inner shelf, both along- and cross-shelf wind components contribute to the transport and, in turn, affect the final stratification. The cross-shelf wind will add to or oppose the upwelling circulation and can extend (offshore wind) or terminate (onshore) the reach of an upwelling cell to connect the midshelf to the surfzone (Figure 5-25). This 
result demonstrates that it is critical to know both wind components to predict the wind-driven cross-shelf circulation over a stratified shelf.

\subsection{In Conclusion}

This thesis has demonstrated that both vertical and horizontal density gradients are key to determining the effectiveness of cross-shelf winds at driving transport across the inner shelf. The nonlinear relationship between the density and velocity fields means that neither can be understood in isolation, and this thesis has made progress towards quantifying the interaction between the two. The modeling and observational analyses in this thesis are the first to address the asymmetry in the response of a stratified inner shelf to onshore and offshore wind stresses, and the measurements from Martha's Vineyard are the first observational evidence for the asymmetry in the response to upwelling and downwelling wind stresses predicted by the $2 \mathrm{D}$ numerical model of Austin and Lentz (2002).

However, the current understanding of these processes is incomplete and results so far point towards a few key topics for future work. Modeling results from sections 5.3 to 5.5 demonstrated that on a well mixed or weakly stratified inner shelf, the cross-shelf density gradient is the variable that scales transport efficiency. So far, an analysis of the cross-shelf density gradients during unstratified conditions in the SWWIM data set has generated suggestive but not statistically significant results. Further investigation of the SWWIM data set or others like it is needed to determine the relative contributions of the vertical and horizontal density gradients.

Preliminary results on the effect of cross-shelf wind stress on the growth of the inner shelf (section 5.6.5) clearly point to the need for further investigation of the processes and scales that set the location and strength of upwelling under combined cross- and along-shelf wind forcing. In addition, the connectivity between inner and mid shelf streamlines (section 5.6.2) depends on the relative strengths of the crossand along-shelf wind stress components. The cross-shelf component affects whether 
the upwelling zone is a barrier to cross-shelf transport.

The final direction for future research is an investigation of the influence of stratification on Lagrangian particle transport. Work should focus on both passive and active tracers, and eventually progress towards understanding the movement of larvae, nutrients, sediments and pollutants across the inner shelf. The broad application of our newfound physical insight to topics related to human and environmental health is the underlying goal behind this and nearly all investigations of nominally physical problems. 


\section{Bibliography}

Adams, C. E. and G. L. Weatherly, 1981: Some effects of suspended sediment stratification on an oceanic bottom boundary layer. J. Geophys. Res., 86, 4161-4172.

Allen, J. S., P. A. Newberger, and J. Federiuk, 1995: Upwelling circulation on the Oregon continental shelf. Part I: Response to idealized forcing. J. Phys. Oceanogr., 25 (8), 1843-1866, URL http: //dx .doi .org/10.1175/1520-0485 (1995) 025<1843:UCOTOC>2 . 0 . C0; 2.

Austin, J. A., 1998: Wind-driven circulation on a shallow stratified shelf. Ph.D. thesis, Massachusetts Institute of Technology/Woods Hole Oceanographic Institution Joint Program in Oceanography/Applied Ocean Science and Engineering.

Austin, J. A. and S. J. Lentz, 2002: The inner shelf response to wind-driven upwelling and downwelling. Journal of Physical Oceanography, 32, 2171- 2193.

Baumgartner, M. F. and D. M. Fratantoni, 2008: Diel periodicity in both sei whale vocalization rates and the vertical migration of their copepod prey observed from ocean gliders. Limnol. Oceanogr., 53 (5, part 2), 21972209.

Boss, E., W. Slade, and P. Hill, 2009: Effect of particulate aggregation in aquatic environments on the beam attenuation and its utility as a proxy for particulate mass. Optics Express, 17 (11), 9408-9420.

Businger, J. A., J. C. Wyngaard, Y. Izumi, and E. F. Bradley, 1971: Flux-profile relationships in the atmospheric surface layer. J. Atmos. Sci., 28 (2), 181-189, URL http: //dx .doi .org/10.1175/1520-0469(1971) 028<0181:FPRITA>2 . 0.C0; 2.

Cacchione, D., P. Wiberg, J. Lynch, J. Irish, and P. Traykovski, 1999: Estimates of suspended-sediment fluxand bedform activity on the inner portion of the Eel continental shelf. Marine Geology, 154, 8397.

Cacchione, D. A., W. D. Grant, D. E. Drake, and S. M. Glenn, 1987: Stormdominated bottom boundary layer dynamics on the Northern California continental shelf: Measurements and predictions. Journal of Geophysical Research, 92 (C2), 1817-1827, URL http://dx.doi.org/10.1029/JC092iC02p01817. 
Cacchione, D. A., P. D. Thorne, Y. Agrawal, and N. J. Nidzieko, 2008: Time-averaged near-bed suspended sediment concentrations under waves and currents: Comparison of measured and model estimates. Continental Shelf Research, 28, 470484.

Castelao, R., R. Chant, S. Glenn, and O. Schofield, 2010: The effects of tides and oscillatory winds on the subtidal inner-shelf cross-shelf circulation. Journal of Physical Oceanography, 40, 775-788, doi:10.1175/2009JPO4273.1.

Chang, G. C., T. D. Dickey, and A. J. W. III, 2001: Sediment resuspension over a continental shelf during Hurricanes Edouard and Hortense. Journal of Geophysical Research, 106 (C5), 9517-9531.

Crockett, J. and C. Nittrouer, 2004: The sandy inner shelf as a repository for muddy sediment: an example from Northern California. Continental Shelf Research, 24, 5573.

Cudaback, C. N., L. Washburn, and E. Dever, 2005: Subtidal inner-shelf circulation near Point Conception, California. J. Geophys. Res., 110 (C10), C10 007-, URL http://dx.doi.org/10.1029/2004JC002608.

D\&A Instrument Company, 2005: Sediment size effects. Tech. rep., online. URL http://www.d-a-instruments.com/sand_mud.html, accessed April 2, 2012.

Deines, K. L., 1999: Backscatter estimation using broadband acoustic Doppler current profilers. Proceedings IEEE 6th Working Conference on Current Measurements, 249-253.

Dzwonkowski, B., K. Park, and L. Jiang, 2011: Subtidal across-shelf velocity structure and surface transport effectiveness on the Alabama shelf of the northeastern Gulf of Mexico. Journal of Geophysical Research, 116, C10012, doi: 10.1029/2011JC007188.

Ekman, V. W., 1905: On the influence of the earth's rotation on ocean currents. Arkiv Math., Astro. Fys., 2, 1-53.

Estrade, P., P. Marchesiello, A. C. De Verdiere, and C. Roy, 2008: Cross-shelf structure of coastal upwelling: A two dimensional extension of Ekman's theory and a mechanism for inner shelf upwelling shut down. Journal of Marine Research, 66 (5), 589-616, doi:doi:10.1357/002224008787536790.

Fewings, M., 2007: Cross-shelf circulation and momentum and heat balances over the inner continental shelf near Martha's Vineyard, Massachusetts. Ph.D. thesis, Massachusetts Institute of Technology/Woods Hole Oceanographic Institution Joint Program, 267 pp.

Fewings, M., S. J. Lentz, and J. Fredericks, 2008: Observations of cross-shelf flow driven by cross-shelf winds on the inner continental shelf. Journal of Physical Oceanography, 38, 2358-2378. 
Fewings, M. R. and S. J. Lentz, 2011: Summertime cooling of the shallow continental shelf. J. Geophys. Res., 116 (C7), C07015-, URL http://dx.doi.org/10.1029/2010JC006744.

Flierl, G. and R. Ferrari, 2006: Boundary layer turbulence lecture notes. MIT OpenCouseWare, course 12.820, Turbulence in the Ocean and Atmosphere, URL http://ocw.mit.edu.

Ganju, N. K., S. J. Lentz, A. R. Kirincich, and J. T. Farrar, 2011: Complex mean circulation over the inner shelf south of Martha's Vineyard revealed by observations and a high-resolution model. J. Geophys. Res., 116 (C10), C10 036-, URL http://dx.doi.org/10.1029/2011JC007035.

Geyer, W. R., 1993: The importance of suppression of turbulence by stratification on the estuarine turbidity maximum. Estuaries, 16 (1), 113-125.

Glenn, S., C. Jones, M. Twardowski, L. Bowers, J. Kerfoot, J. Kohut, D. Webb, and O. Schofield, 2008: Glider observations of sediment resuspension in a Middle Atlantic Bight fall transition storm. Limnol. Oceanogr., 53 (5, part 2), 21802196.

Glenn, S., et al., 2010: Observations of storm response and sediment transport on the Middle Atlantic Bight continental shelf. Ocean Sciences Meeting, AGU, Portland, OR,, presented at Ocean Sciences Meeting, AGU.

Glenn, S. M. and W. D. Grant, 1987: A suspended sediment stratification correction for combined wave and current flows. J. Geophys Res., 92, 8244-8264.

Goff, J. A., et al., 2005: Detailed investigation of sorted bedforms,or rippled scour depressions, within the Martha's Vineyard Coastal Observatory, Massachusetts. Continental Shelf Research, 25, 461484.

Gostiaux, L. and H. van Haren, 2010: Extracting meaningful information from uncalibrated backscattered echo intensity data. Journal of Atmospheric and Oceanic Technology, 27, 943-949.

Harris, C. and P. Wiberg, 2001: A two-dimensional, time-dependent model of suspended sediment transport and bed reworking for continental shelves. Computers and Geosciences, 27, 675690.

Harris, C. K., B. Butman, and P. Traykovski, 2003: Wintertime circulation and sediment transport in the Hudson shelf valley. Cont. Shelf Res., 23, 801820.

Hoitink, A. J. F. and P. Hoekstra, 2005: Observations of suspended sediment from ADCP and OBS measurements in a mud-dominated environment. Coastal Engineering, 52, 103118.

IMCS, Ocean Modeling Group, 2012: ROMS $>$ start. URL http://www.myroms.org/, accessed 20 March 2012. 
Kantha, L. H. and C. A. Clayson, 1994: An improved mixed layer model for geophysical applications. J. Geophys. Res., 99 (C12), 25 235-25266, URL http://dx.doi.org/10.1029/94JC02257.

Kim, Y. H. and G. Voulgaris, 2003: Estimation of suspended sediment concentration in estuarine environments using acoustic backscatter from an ADCP. Proceedings of the International Conference on Coastal Sediments 2003, URL http: //www.rdinstruments.com/pdfs/Kim_Yong.pdf.

Kirincich, A. R. and J. A. Barth, 2009: Time-varying across-shelf Ekman transport and vertical eddy viscosity on the inner shelf. Journal of Physical Oceanography, 39 (3), 602-620, doi:10.1175/2008JPO3969.1.

Kirincich, A. R., J. A. Barth, B. A. Grantham, B. A. Menge, and J. Lubchenco, 2005: Wind-driven inner-shelf circulation off central Oregon during summer. J. Geophys. Res., 110 (C10), C10S03-, URL http://dx.doi.org/10.1029/2004JC002611.

Kurapov, A. L., J. S. Allen, and G. D. Egbert, 2010: Combined effects of wind-driven upwelling and internal tide on the continental shelf. J. Phys. Oceanogr., 40 (4), 737-756, URL http://dx.doi.org/10.1175/2009JP04183.1.

Lentz, S. J., 1995: Sensitivity of the inner-shelf circulation to the form of the eddy viscosity profile. J. Phys. Oceanogr., 25 (1), 19-28, URL http: //dx .doi .org/10.1175/1520-0485(1995) 025<0019 : SOTISC>2 . 0 . C0; 2.

Lentz, S. J., 2001: The influence of stratification on the wind-driven cross-shelf circulation over the North Carolina shelf. Journal of Physical Oceanography, 31, 27492760 .

Lentz, S. J., 2008: Seasonal variations in the circulation over the Middle Atlantic Bight continental shelf. J. Phys. Oceanogr., 38 (7), 1486-1500, URL http://dx.doi.org/10.1175/2007JP03767.1.

Lentz, S. J. and D. C. Chapman, 2004: The importance of nonlinear cross-shelf momentum flux during wind-driven coastal upwelling. Journal of Physical Oceanography, 34, $2444-2457$.

Lentz, S. J., M. Fewings, P. Howd, J. Fredericks, and K. Hathaway, 2008: Observations and a model of undertow over the inner continental shelf. Journal of Physical Oceanography, 38 (11), 2341-2357, doi:10.1175/2008JPO3986.1, URL http://journals.ametsoc.org/doi/abs/10.1175/2008JP03986.1.

Lentz, S. J. and M. R. Fewings, 2011: The wind- and wave-driven inner-shelf circulation. Annu. Rev. Marine. Sci., 4 (1), 317-343, URL http://dx.doi.org/10.1146/annurev-marine-120709-142745. 
Li, Z. and R. H. Weisberg, 1999a: West Florida continental shelf response to upwelling favorable wind forcing 2. Dynamics. J. Geophys. Res., 104 (C10), 23 427-23 442, URL http://dx.doi.org/10.1029/1999JC900205.

Li, Z. and R. H. Weisberg, 1999b: West Florida shelf response to upwelling favorable wind forcing: Kinematics. J. Geophys. Res., 104 (C6), 13507-13 527, URL http://dx.doi.org/10.1029/1999JC900073.

Lynch, J., J. Irish, C. Sherwood, and Y. Agrawal, 1994: Determining suspended sediment particle size information from acoustical and optical backscatter measurements. Continental Shelf Research, 14 (1011), 11391164.

Madsen, O. S., 1994: Spectral wave-current bottom boundary layer flows. 24th Coastal Engineering Conference, Kobe, Japan,, Am. Soc. of Civ. Eng.

Mellor, G. L. and T. Yamada, 1974: A hierarchy of turbulence closure models for planetary boundary layers. J. Atmos. Sci., 31, 1791-1806.

Mellor, G. L. and T. Yamada, 1982: Development of a turbulence closure model for geophysical fluid problems. Rev. Geophys., 20 (4), 851-875, URL http://dx.doi.org/10.1029/RG020i004p00851.

Monin, A. S. and A. M. Obukhov, 1954: Basic laws of turbulent mixing in the ground layer of the atmosphere. Akad. Nauk SSSR Geofiz. Inst. Tr., 151, 163-187.

Pawlowicz, R., B. Beardsley, and S. Lentz, 2002: Classical tidal harmonic analysis including error estimates in MATLAB using T_TIDE. Computers and Geosciences, 28, 929-937.

Pollard, R. T., P. B. Rhines, and R. O. R. Y. Thompson, 1973: The deepening of the wind-mixed layer. Geophysical Fluid Dynamics, 3 (1), 381-404, doi:10.1080/03091927208236105, URL http://www.tandfonline.com/doi/abs/10.1080/03091927208236105.

Price, J. F., R. A. Weller, and R. Pinkel, 1986: Diurnal cycling: Observations and models of the upper ocean response to diurnal heating, cooling, and wind mixing. J. Geophys. Res., 91 (C7), 8411-8427, URL http://dx.doi.org/10.1029/JC091iC07p08411.

Rouse, H., 1937: Modern conceptions of the mechanics of fluid turbulence. Transactions of the American Society of Civil Engineering, 102, 463-554.

Rouse, H., 1961: Fluid mechanics for hydraulic engineers. Dover Publications, New York,.

Scully, M. E. and C. T. Friedrichs, 2003: The influence of asymmetries in overlying stratification on near-bed turbulence and sediment suspension in a partially mixed estuary. Ocean Dynamics, 53, 208219. 
Shchepetkin, A. F. and J. C. McWilliams, 2005: The regional oceanic modeling system (ROMS): a split-explicit, free-surface, topography-following-coordinate oceanic model. Ocean Modelling, 9 (4), 347 - 404, doi:10.1016/j.ocemod.2004.08.002, URL http://www.sciencedirect.com/science/article/pii/S1463500304000484.

Shearman, R. K. and S. J. Lentz, 2004: Observations of tidal variability on the new england shelf. J. Geophys. Res., 109 (C6), C06010, URL http://dx.doi.org/10.1029/2003JC001972.

Sherwood, C. R., B. Butman, D. A. Cacchione, D. E. Drake, T. F. Gross, R. . W. Sternberg, P. L. Wiberg, and A. J. W. III., 1994: Sediment-transport events on the northern california continental shelf during the 1990-1991 STRESS experiment. Continental Shelf Research, 14 (10111), 1063-1099.

Simpson, J. H., J. Brown, J. Matthews, and G. Allen, 1990: Tidal straining, density currents, and stirring in the control of estuarine stratification. Estuaries, 13 (2), 125-132, URL http://www.jstor.org/stable/1351581.

Smith, J. D., 1977: Modeling of sediment transport on continental shelves, 539-577. Wiley-Interscience, New York.

Souza, A. J., T. D. Dickey, and G. C. Chang, 2001: Modeling water column structure and suspended particulate matter on the Middle Atlantic continental shelf during the passages of Hurricanes Edouard and Hortense. Journal of Marine Research, 59, 10211045 .

Stokes, G. G., 1847: On the theory of oscillatory waves. Trans. Cambridge Philos. Soc., 8, 441-455.

Storlazzi, C. and B. Jaffe, 2002: Flow and sediment suspension events on the inner shelf of central California. Marine Geology, 181, 195-213.

Styles, R. and S. M. Glenn, 2005: Long-term sediment mobilization at LEO-15. J. Geophys. Res., 110 (C04S90), C04S90.

Thorne, P. D. and D. M. Hanes, 2002: A review of acoustic measurement of smallscale sediment processes. Continental Shelf Research, 22, 603632.

Thorne, P. D., P. J. Hardcastle, and R. L. Soulsby, 1993: Analysis of acoustic measurements of suspended sediments. Journal of Geophysical Research, vol. 98 (C1), 899-910.

Tilburg, C. E., 2003: Across-shelf transport on a continental shelf: Do across-shelf winds matter? Journal of Physical Oceanography, 33, 2675-2688.

Traykovski, P., A. Hay, J. Irish, and J. Lynch, 1999: Geometry, migration and evolution of wave orbital scale ripples at LEO-15. Journal of Geophysical Research, 104, 15051524 . 
Traykovski, P., M. D. Richardson, L. A. Mayer, and J. D. Irish, 2007: Mine burial experiments at the Martha's Vineyard Coastal Observatory. IEEE Journal of Oceanic Engineering, 32 (1), 150 - 166, doi:10.1109/JOE.2007.890956.

Urick, R. J., 1948: The absorption of sound in suspensions of irregular particles. J. Acoust. Soc. Am., 20 (3), 283-289.

Urick, R. J., 1983: Principles of Underwater Sound. 3d ed., McGraw-Hill, Inc., New York,, $423 \mathrm{pp}$.

Villaret, C. and J. H. Trowbridge, 1991: Effects of stratification by suspended sediments on turbulent shear flows. Journal of Geophysical Research, 96 (C6), 10,65910,680 .

Wall, G., E. Nystrom, and S. Litten, 2006: Use of an ADCP to compute suspendedsediment discharge in the tidal Hudson River, New York. Tech. rep., U.S. Geological Survey, 26 pp.

Weatherly, G. L. and P. J. Martin, 1978: On the structure and dynamics of the oceanic bottom boundary layer. Journal of Physical Oceanography, 8, 557-570.

Weisberg, R. H., Z. Li, and F. Muller-Karger, 2001: West Florida shelf response to local wind forcing: April 1998. J. Geophys. Res., 106 (C12), 31 239-31 262, URL http://dx.doi.org/10.1029/2000JC000529.

Wijesekera, H. W., J. S. Allen, and P. A. Newberger, 2003: Modeling study of turbulent mixing over the continental shelf: Comparison of turbulent closure schemes. Journal of Geophysical Research, 108 (C3), 3103, doi:10.1029/2001JC001234.

Winterwerp, J. C., 2001: Stratification effects by cohesive and noncohesive sediment. Journal of Geophysical Research, 106 (C10), 22,559-22,574.

Wright, L. D., J. D. Boon, S. Kim, and J. List, 1991: Modes of cross-shore sediment transport on the shoreface of the Middle Atlantic Bight. Marine Geology, 96, 19-51.

Zhang, W. G., G. G. Gawarkiewicz, D. J. McGillicuddy, and J. L. Wilkin, 2011: Climatological mean circulation at the New England shelf break. J. Phys. Oceanogr., 41 (10), 1874-1893, URL http://dx.doi.org/10.1175/2011JP04604.1. 